\title{
Precision Measurement of the Mass and Width of the W Boson at CDF
}

\author{
Sarah Alam Malik \\ University College London
}

Submitted to University College London in fulfilment of the requirements for the award of the degree of Doctor of Philosophy.

September 2009 
I, Sarah Alam Malik, confirm that the work presented in this thesis is my own. Where information has been derived from other sources, I confirm that this has been indicated in the thesis. 


\begin{abstract}
A precision measurement of the mass and width of the $\mathrm{W}$ boson is presented. The $\mathrm{W}$ bosons are produced in proton antiproton collisions occurring at a centre of mass energy of $1.96 \mathrm{TeV}$ at the Tevatron accelerator. The data used for the analyses is collected by the Collider Detector at Fermilab (CDF) and corresponds to an average integrated luminosity of $350 \mathrm{pb}^{-1}$ for the $\mathrm{W}$ width analysis for the electron and muon channels and an average integrated luminosity of $2350 \mathrm{pb}^{-1}$ for the $\mathrm{W}$ mass analysis.

The mass and width of the $\mathrm{W}$ boson is extracted by fitting to the transverse mass distribution, with the peak of the distribution being most sensitive to the mass and the tail of the distribution sensitive to the width. The $\mathrm{W}$ width measurement in the electron and muon channels is combined to give a final result of $2032 \pm 73 \mathrm{MeV}$.

The systematic uncertainty on the $\mathrm{W}$ mass from the recoil of the $\mathrm{W}$ boson against the initial state gluon radiation is discussed. A systematic study of the recoil in $\mathrm{Z} \rightarrow e^{+} e^{-}$ events where one electron is reconstructed in the central calorimeter and the other in the plug calorimeter and its effect on the $\mathrm{W}$ mass is presented for the first time in this thesis.
\end{abstract}




\section{Acknowledgements}

I would like to thank the entire UCL CDF team; Dan Beecher, Ilija Bizjak, Emily Nurse, Troy Vine, Dave Waters and in particular my supervisor Mark Lancaster. I would especially like to thank Emily Nurse for all her help and support and Simon Dean for taking out so much time to help me when I needed it most.

I would like to thank my family for being there through it all, especially my parents who have been asking me everyday for the past 2 years when I'm going to finish!. Naeem chacha for the endless prayers, Ayesha, for constantly making me defend my decision to study Physics, Fatima, for being the real doctor and Abdul-Rehman, I still haven't forgiven you for not choosing to study Physics at University.

Above all, I would like to thank Dawood for being the one that kept me afloat through all the ups and downs. I don't know how you put up with me through all my moodiness but I couldn't have done it without your love, support and patience. 
vi

\section{Contents}

$\begin{array}{ll}\text { Abstract } & \text { ii }\end{array}$

Acknowledgements $\quad$ v

Contents $\quad$ vi

List of Figures $\quad x$

List of Tables $\quad$ xviii

1. The Standard Model 1

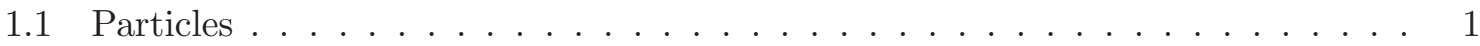

1.2 Electromagnetic Interaction . . . . . . . . . . . . . . . . . . 3

1.3 Strong Interaction $\ldots \ldots \ldots \ldots \ldots$

1.4 Electroweak Interaction . . . . . . . . . . . . . . . . . . 5

1.5 Beyond the Standard Model . . . . . . . . . . . . . . . . 8

2. The Tevatron and CDF 11

2.1 The Tevatron . . . . . . . . . . . . . . . . . . . 11

2.2 Tevatron performance and luminosity . . . . . . . . . . . . . . . 13

2.3 The CDF Detector . . . . . . . . . . . . . . . . 15

2.3.1 CDF Coordinate System . . . . . . . . . . . . . . 16

2.3 .2 Tracking System . . . . . . . . . . . . . . . . . 17

2.3.3 Calorimeter System . . . . . . . . . . . . . . 20 
2.3.4 Muon Chambers . . . . . . . . . . . . . . . . . . 24

2.4 The Trigger System . . . . . . . . . . . . . . . . . . . 26

2.4 .1 Electron Trigger . . . . . . . . . . . . . . . . 27

2.4 .2 Muon Trigger . . . . . . . . . . . . . . . . . . . . . . . 28

3. The Mass and Width of the W Boson 29

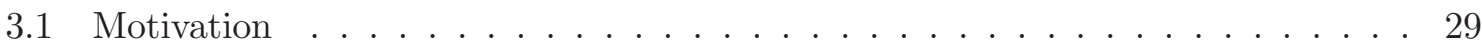

3.1 .1 W Mass . . . . . . . . . . . . . . . . . . . . . . 29

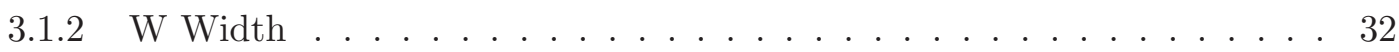

3.2 Measurement Strategy . . . . . . . . . . . . . . . . . . . 34

4. Event Selection 44

4.1 Electron Selection . . . . . . . . . . . . . . . . . . 45

4.1.1 Electron Reconstruction . . . . . . . . . . . . . . . 47

4.2 Muon Selection . . . . . . . . . . . . . . . . . . 47

4.3 Event Cuts . . . . . . . . . . . . . . . . . . . . . . . 49

5. Event Generation $\quad 52$

5.1 Parton Distribution Functions $(\mathrm{PDF}) \ldots \ldots \ldots \ldots \ldots$

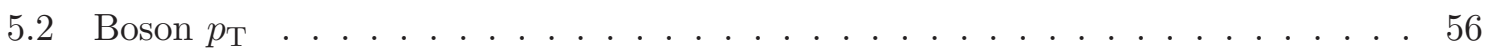

5.2 .1 Determination of $p_{T}^{Z}$ and $p_{T}^{W} \ldots \ldots \ldots \ldots \ldots$

$5.2 .2 p_{T}^{W}$ Systematic . . . . . . . . . . . . . . . . 58

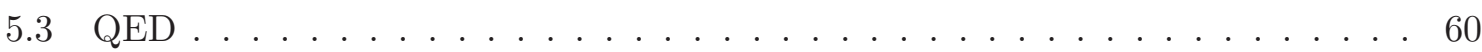

5.4 Electroweak Box Diagrams . . . . . . . . . . . . . . 62

$\begin{array}{lll}\text { 6. } & \text { Event Simulation } & 64\end{array}$

6.1 Silicon . . . . . . . . . . . . . . . . . . . 66

6.1 Systematic Uncertainty . . . . . . . . . . . . . . 67

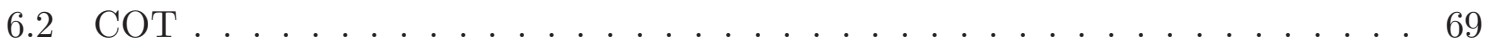

6.2.1 Momentum Scale and Resolution . . . . . . . . . . . . . . . . 70

6.2 .2 Systematic Uncertainty . . . . . . . . . . . . . . . . . 72 
6.3 Material Scale. . . . . . . . . . . . . . . . . . . . 73

$6.4 \mathrm{ToF}$ and Solenoid . . . . . . . . . . . . . . . . . . . . 74

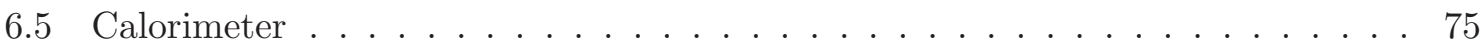

6.5.1 CEM Scale and Resolution _. . . . . . . . . . . . 76

6.5.2 Muon Energy Simulation . . . . . . . . . . . . . . . . 80

6.5.3 Underlying Energy Simulation . . . . . . . . . . . . . . . . 80

6.5.4 Calorimeter Non-linearity . . . . . . . . . . . . . . . 81

6.6 Acceptances and Efficiencies . . . . . . . . . . . . . . . . 81

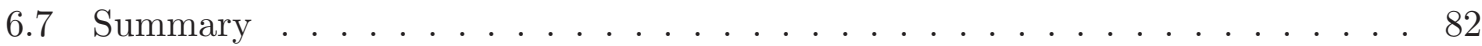

$\begin{array}{lll}\text { 7. } & \text { Recoil Reconstruction and Simulation } & 83\end{array}$

7.1 Recoil reconstruction . . . . . . . . . . . . . . . . . 85

7.1 .1 Knockout Region . . . . . . . . . . . . . . . . 86

$7.1 .2 \phi_{U}$ Modulation Correction . . . . . . . . . . . . . 88

7.2 Recoil Simulation . . . . . . . . . . . . . . . . . . . . . . 91

7.2.1 Knockout Region Simulation . . . . . . . . . . . . . . . . . 92

7.2 .2 Simulation of $U_{\text {BREM }} \ldots \ldots \ldots \ldots \ldots \ldots$

7.2 .3 The Recoil Model . . . . . . . . . . . . . . . . . . . . . . . 97

7.2 .4 Recoil Fits . . . . . . . . . . . . . . . . 107

7.3 Recoil Comparison . . . . . . . . . . . . . . . . . 116

7.4 Recoil Systematic . . . . . . . . . . . . . . . . 117

8. Recoil in Central-Plug Events 133

8.1 Event Selection . . . . . . . . . . . . . . . . . . . 134

8.2 Event Reconstruction . . . . . . . . . . . . . . . . 135

8.2.1 Plug Knockout Region . . . . . . . . . . . . . . . . 135

8.3 Event Simulation . . . . . . . . . . . . . . . . . . . 141

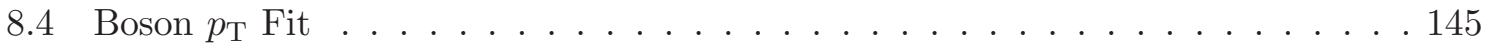

8.5 Knockout Region Simulation . . . . . . . . . . . . . . . . 146

8.6 Backgrounds . . . . . . . . . . . . . . . . . . . . 148 
8.6 .1 Non-QCD Backgrounds . . . . . . . . . . . . . . . . . . 149

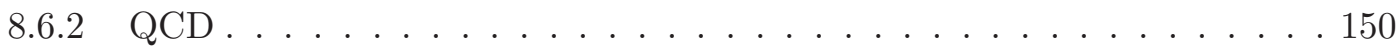

8.7 Recoil Simulation . . . . . . . . . . . . . . . . . 153

8.8 Results . . . . . . . . . . . . . . . . . . . 157

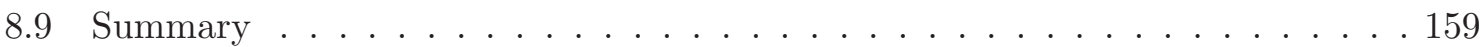

$\begin{array}{lll}\text { 9. } & \text { Backgrounds } & 160\end{array}$

9.1 Electroweak Backgrounds . . . . . . . . . . . . . . . . 161

9.1.1 Electroweak Backgrounds in $\mathrm{W} \rightarrow e \nu \ldots \ldots \ldots 1$

9.1.2 Electroweak Background in $\mathrm{Z} \rightarrow e^{+} e^{-} \ldots \ldots \ldots$. . . . . . . . 162

9.2 Diffractive Background . . . . . . . . . . . . . . . . . 163

9.3 QCD Background . . . . . . . . . . . . . . . . . . 164

$\begin{array}{lll}\text { 10. } & \text { Results } & 173\end{array}$

10.1 W Width . . . . . . . . . . . . . . . . . . . . . 173

$10.2 \mathrm{~W}$ Mass . . . . . . . . . . . . . . . . . . . . . 177

10.3 Summary . . . . . . . . . . . . . . . . . . . . . 180

$\begin{array}{ll}\text { References } & 181\end{array}$ 


\section{List of Figures}

2.1 The various stages of production and acceleration of protons and antiprotons. . 13

2.2 The peak luminosity of a store as a function of time for the period of Run II. . 14

2.3 The integrated luminosity as a function of time for the period of Run II. . . . 15

2.4 An elevation view of one half of the CDF detector. . . . . . . . . . . . 16

2.5 The CDF coordinate system. . . . . . . . . . . . . . 17

2.6 Superlayers of the COT, alternating between stereo and axial superlayers. . . . 19

2.7 A schematic diagram showing where different particle types deposit their energy in the CDF calorimeter system. . . . . . . . . . . . . . 20

2.8 A schematic diagram of a central calorimeter wedge showing the ten electromagnetic calorimeter towers. . . . . . . . . . . . . . . 22

2.9 Schematic diagram of the proportional strip chamber (CES) in the central

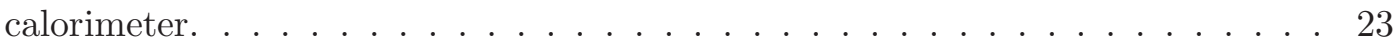

2.10 Schematic diagram of the sub-detectors comprising the muon chamber and their coverage in $\eta$ and $\phi . \ldots \ldots \ldots \ldots \ldots \ldots \ldots$

3.1 Radiative corrections to the $\mathrm{W}$ boson propagator from the (a) $t \bar{b}$ loop and (b) the Higgs boson loop. . . . . . . . . . . . . . . . . . . 30

3.2 The mass of the $\mathrm{W}$ boson as measured by experiments at LEP2 and the Tevatron. 31

3.3 Constraints on the Higgs boson mass as a function of the $\mathrm{W}$ boson and top quark masses. . . . . . . . . . . . . . . . . . . . . . . 33

3.4 The $\Delta \chi^{2}$ from all electroweak precision measurements as a function of the Higgs mass. . . . . . . . . . . . . . . . . . . . . . 33 
3.5 The width of the $\mathrm{W}$ boson as measured by the LEP and Tevatron experiments. 34

3.6 Feynman diagram for (a) lowest order production and decay of a $\mathrm{W}^{+}$boson and (b) next to leading order $\mathrm{W}^{+}$boson production. . . . . . . . . . . . 36

3.7 A diagram showing the direction of momenta and spin for the colliding partons

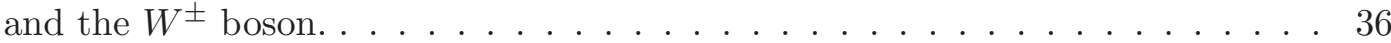

3.8 The Collins-Soper frame. . . . . . . . . . . . . . . . . . . . . . 37

3.9 The transverse mass spectrum and the electron transverse momentum distri-

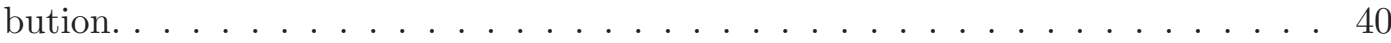

3.10 A comparison of the shape of the Breit-Wigner width component and the detector resolution described by a Gaussian. . . . . . . . . . . . . . . . . . 41

3.11 The $M_{T}$ distribution for different input values for the $\mathrm{W}$ width in the simulation. 41

5.1 The production of a $\mathrm{W}$ boson with initial state gluon radiation. . . . . . . . . 53

5.2 Feynman diagrams for the radiative corrections to $\mathrm{W}$ production and decay simulated by the Berends and Kleiss program. . . . . . . . . . . . . . . 53

5.3 Fitted $\Gamma_{W}$ for the CTEQ6 PDF set. . . . . . . . . . . . 55

5.4 The measured $p_{T}^{Z}$ distributions compared to the best fit MC prediction for (a) $\mathrm{Z} \rightarrow e^{+} e^{-}$data and $(\mathrm{b}) \mathrm{Z} \rightarrow \mu^{+} \mu^{-}$data. . . . . . . . . . . . . 57

5.5 The $1 \sigma$ and $2 \sigma$ correlation contours for $g_{2}$ and $B \ldots \ldots \ldots \ldots$

5.6 Feynman diagrams for the $\mathrm{W}, \mathrm{Z}$ box diagrams in the $p \bar{p} \rightarrow l \nu$ process. . . . . 63

6.1 Schematic diagram showing the sub-detectors traversed by particles at CDF. . 65

6.2 The evolution of an electromagnetic shower in $\mathrm{Z} \rightarrow e^{+} e^{-}$events. . . . . . . . 68

6.3 The $\Delta \rho$ distribution taken from $\mathrm{W} \rightarrow \mu \nu$ CdfSim events for muon tracks. . . . 71

6.4 Fit to the $\mathrm{Z}$ invariant mass distribution in $\mathrm{Z} \rightarrow \mu^{+} \mu^{-}$candidate events to obtain the momentum scale and resolution. . . . . . . . . . . . . . . . 72

6.5 The energy fraction deposited in the CEM as a function of the incident electron energy and incident photon energy. . . . . . . . . . . . . . . 76

6.6 The variation of the $\langle E / p\rangle$ as a function of time in $\mathrm{W} \rightarrow e \nu$ events. . . . . . 78

6.7 A fit to the $E / p$ distribution in $\mathrm{W} \rightarrow e \nu$ events to determine the calorimeter scale and the uncorrelated contribution to the detector resolution. . . . . . . 78 
6.8 A fit to the $M_{e e}$ distribution in $\mathrm{Z} \rightarrow e^{+} e^{-}$events to determine $\kappa^{\text {uncorr }}$ and $S^{\mathrm{CEM}} .79$

7.1 The $3 \times 3$ tower region around the electron tower. . . . . . . . . . . 87

7.2 The energy (in $\mathrm{MeV}$ ) in the $3 \times 3$ tower region around the muon tower. . . . . . 88

7.3 The energy (in $\mathrm{MeV}$ ) in the $3 \times 3$ tower region around the electron tower. . . . 89

7.4 The $3 \times 3$ tower region around the muon tower. . . . . . . . . . . . . . 89

7.5 The modulation in $\phi_{U}$ after correcting for the beam offset. . . . . . . . . . . . 90

7.6 The modulation in $\phi_{U}$ in $\mathrm{W} \rightarrow e \nu$ events before and after the plug alignment

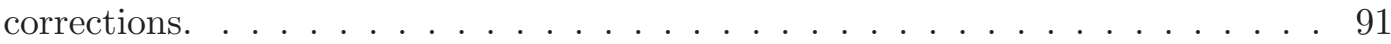

7.7 The average electromagnetic energy in towers around the electron. . . . . . . 93

7.8 Underlying energy in a 7 -tower pseudo-cluster. . . . . . . . . . . . . . 95

7.9 Underlying energy in a pseudo-cluster as a function of $U_{\|}$in two ranges of luminosity in $\mathrm{W} \rightarrow e \nu$ events for the $\mathrm{W}$ mass analysis. . . . . . . . . . . . . 96

7.10 The distribution of the bremsstrahlung contribution to the recoil for $\mathrm{W} \rightarrow e \nu$ and $\mathrm{W} \rightarrow \mu \nu$ simulation events in the $\mathrm{W}$ mass analysis. . . . . . . . . . . . 97

$7.11 \Sigma E_{\mathrm{T}}$ distributions for (a) $\mathrm{Z} \rightarrow e^{+} e^{-}$and (b) $\mathrm{Z} \rightarrow \mu^{+} \mu^{-}$data compared to the simulation using the best fit $\Sigma E_{\mathrm{T}}$ parameters in the $\mathrm{W}$ width analysis. . . . . . 101

7.12 The variation of $\left\langle\Sigma E_{\mathrm{T}}\right\rangle$ with the transverse momentum of the $\mathrm{Z}$ boson in data and simulation for (a) $\mathrm{Z} \rightarrow e^{+} e^{-}$events and (b) $\mathrm{Z} \rightarrow \mu^{+} \mu^{-}$events. . . . . . . 102

7.13 Comparison of the instantaneous luminosity distribution in $\mathrm{Z} \rightarrow e^{+} e^{-}$events for the $370 \mathrm{pb}^{-1}$ data sample used in the $\mathrm{W}$ width analysis and the $2.4 \mathrm{fb}^{-1}$ data sample used in the $\mathrm{W}$ mass analysis. . . . . . . . . . . . . . . . 102

$7.14 \Sigma E_{\mathrm{T}}$ distributions for (a) $\mathrm{Z} \rightarrow e^{+} e^{-}$and (b) $\mathrm{Z} \rightarrow \mu^{+} \mu^{-}$data compared to the simulation using the best fit $\Sigma E_{\mathrm{T}}$ parameters in the W mass analysis. . . . . . 104

7.15 The dependence of the mean and standard deviation of the $\Sigma E_{\mathrm{T}}$ on luminosity and boson $p_{\mathrm{T}}$ in $\mathrm{Z} \rightarrow e^{+} e^{-}$events. . . . . . . . . . . . . . . 105

7.16 The dependence of the mean and standard deviation of the $\Sigma E_{\mathrm{T}}$ on luminosity and boson $p_{\mathrm{T}}$ in $\mathrm{Z} \rightarrow \mu^{+} \mu^{-}$events. . . . . . . . . . . . . 106

7.17 The fit to the (a) $\sigma\left(U_{x}\right)$ and (b) $\sigma\left(U_{y}\right)$ distributions as a function of $\Sigma E_{\mathrm{T}}$ in minimum-bias data for the $\mathrm{W}$ width analysis. . . . . . . . . . . . . 107 
7.18 The fit to the (a) $\sigma\left(U_{x}\right)$ and (b) $\sigma\left(U_{y}\right)$ distributions as a function of $\Sigma E_{\mathrm{T}}$ in minimum-bias data for the $\mathrm{W}$ mass analysis. . . . . . . . . . . . . . . . 108

7.19 The distributions of (a) and (b) $\left\langle U_{1}\right\rangle$ vs. $p_{T}^{Z}$, (c) and (d) $\sigma\left(U_{1}\right)$ vs. $p_{T}^{Z}$, (e) and (f) $\sigma\left(U_{2}\right)$ vs. $p_{T}^{Z}$ in $\mathrm{Z} \rightarrow e^{+} e^{-}$and $\mathrm{Z} \rightarrow \mu^{+} \mu^{-}$events compared to the best fit simulation in the $\mathrm{W}$ width analysis. . . . . . . . . . . . . . . 111

7.20 Recoil distributions of (a) and (b) $U_{1}$, (c) and (d) $U_{2}$ and (e) and (f) $U$ for the $\mathrm{Z} \rightarrow e^{+} e^{-}$and $\mathrm{Z} \rightarrow \mu^{+} \mu^{-}$channels compared to the best fit simulation in the W width analysis. . . . . . . . . . . . . . . . . . 112

7.21 The distributions of (a) and (b) $\left\langle U_{1}\right\rangle$ vs. $p_{T}^{Z}$, (c) and (d) $\sigma\left(U_{1}\right)$ vs. $p_{T}^{Z}$, (e) and (f) $\sigma\left(U_{2}\right)$ vs. $p_{T}^{Z}$ in $\mathrm{Z} \rightarrow e^{+} e^{-}$and $\mathrm{Z} \rightarrow \mu^{+} \mu^{-}$events compared to the best fit simulation in the $\mathrm{W}$ mass analysis. . . . . . . . . . . . . . . 113

7.22 Distributions showing the dependence of the $U_{1}$ and $U_{2}$ resolutions on luminosity in the data compared to the simulation using the best fit recoil parameters in the $\mathrm{W}$ mass analysis. . . . . . . . . . . . . . . . . . . . . 114

7.23 Constant $\chi^{2}$ contour of the $\left\langle U_{1}\right\rangle$ vs $p_{T}^{Z}$ distribution in $\mathrm{Z} \rightarrow e^{+} e^{-}$events as the two response parameters $P_{1}$ and $P_{2}$ are varied in the $\mathrm{W}$ mass analysis. . . . . 114

7.24 Recoil distributions of $U, U_{1}$ and $U_{2}$ for the $\mathrm{Z} \rightarrow e^{+} e^{-}$and $\mathrm{Z} \rightarrow \mu^{+} \mu^{-}$channels compared to the best fit simulation in the $\mathrm{W}$ mass analysis. . . . . . . . . . 115

7.25 Distributions of $U, U_{\|}$and $U_{\perp}$ in $\mathrm{W} \rightarrow e \nu$ and $\mathrm{W} \rightarrow \mu \nu$ data compared to the simulation for the $\mathrm{W}$ width analysis. . . . . . . . . . . . . 123

7.26 Distribution of $\not_{T}$ in (a) $\mathrm{W} \rightarrow e \nu$ and (b) $\mathrm{W} \rightarrow \mu \nu$ events compared to the simulation for the $\mathrm{W}$ width analysis. . . . . . . . . . . . . . . . . . 124

7.27 Variation of the $\left\langle U_{\|}\right\rangle$with $M_{T}$ and $U$ in the $\mathrm{W} \rightarrow e \nu$ and $\mathrm{W} \rightarrow \mu \nu$ data compared to the simulation in the $\mathrm{W}$ width analysis. . . . . . . . . . . . . . 124

7.28 Variation of the $\left\langle U_{\|}\right\rangle$with $\Delta \phi(U, l)$ and $\sigma\left(U_{\perp}\right)$ with $U$ in the $\mathrm{W} \rightarrow e \nu$ and $\mathrm{W} \rightarrow \mu \nu$ data compared to the simulation in the $\mathrm{W}$ width analysis. . . . . . 125

7.29 Distributions of $U, U_{\|}$and $U_{\perp}$ in $\mathrm{W} \rightarrow e \nu$ and $\mathrm{W} \rightarrow \mu \nu$ data compared to the simulation for the $\mathrm{W}$ mass analysis. . . . . . . . . . . . . . 126

7.30 Variation of the $\left\langle U_{\|}\right\rangle$with $M_{T}$ and $U$ in the $\mathrm{W} \rightarrow e \nu$ and $\mathrm{W} \rightarrow \mu \nu$ data compared to the simulation in the $\mathrm{W}$ mass analysis. . . . . . . . . . . . . 127 
7.31 Variation of the $\left\langle U_{\|}\right\rangle$with $\Delta \phi(U, l)$ and $\sigma\left(U_{\perp}\right)$ with $U$ in the $\mathrm{W} \rightarrow e \nu$ and $\mathrm{W} \rightarrow \mu \nu$ data compared to the simulation in the $\mathrm{W}$ mass analysis. . . . . . . 128

7.32 Variation of the resolution of $U$ with $\Sigma E_{\mathrm{T}}$ in the $\mathrm{W} \rightarrow e \nu$ data compared to the simulation in the $\mathrm{W}$ mass analysis. . . . . . . . . . . . . . . 128

7.33 Distribution of $E_{T}$ in (a) $\mathrm{W} \rightarrow e \nu$ and (b) $\mathrm{W} \rightarrow \mu \nu$ events compared to the simulation for the $\mathrm{W}$ mass analysis. . . . . . . . . . . . . . . . . 129

7.34 The spread of the $\chi^{2}$ of three $\mathrm{W}$ recoil distributions and the three fitted $\mathrm{Z}$ distributions when the electron and the muon covariance matrix are sampled in turn. . . . . . . . . . . . . . . . . . . . . 129

7.35 The effect on the $\chi^{2}$ of some of the recoil distribution in $\mathrm{Z} \rightarrow e^{+} e^{-}$events when using the recoil parameters optimised on the $U$ distribution. . . . . . . . . . . 130

7.36 The effect on the $\chi^{2}$ of some of the recoil distribution in $\mathrm{W} \rightarrow e \nu$ events when using the recoil parameters optimised on the $U$ distribution. . . . . . . . . . . 130

7.37 The uncertainty on the shape of the U distribution from statistics, the error on the recoil parameters (Recoil), boson $p_{\mathrm{T}}$ determination $(\mathrm{Pt})$ and backgrounds (Bcg) for (a) $\mathrm{W} \rightarrow e \nu$ and (b) $\mathrm{W} \rightarrow \mu \nu$ events in the $\mathrm{W}$ width analysis. . . . . 131

7.38 Spread of $M_{W}$ values obtained when fitting to $M_{T}$ distributions obtained using different sets of recoil parameters obtained from sampling the $6 \times 6$ covariance matrix in (a) $\mathrm{W} \rightarrow e \nu$ and (b) $\mathrm{W} \rightarrow \mu \nu$ events in the $\mathrm{W}$ mass analysis. . . . . 131

7.39 The $U_{\|}$distribution for backgrounds contributing to the electron channel in the $\mathrm{W}$ mass analysis. . . . . . . . . . . . . . . . . . . . . 132

8.1 The segmentation in $(\eta, \phi)$ of the plug calorimeter (only a quarter of the detector is shown in $\phi) \ldots \ldots \ldots \ldots$. . . . . . . . . . . . . . . . . . . . . . . . . .

8.2 The energy deposited (in $\mathrm{MeV}$ ) in the knockout region for events where the plug electron is reconstructed in a region of the calorimeter that has a 24 -wedge segmentation in $\phi . \ldots \ldots \ldots \ldots \ldots$. . . . . . . . . . . . . . . . . . . . . . . . . . . . .

8.3 The energy deposited (in $\mathrm{MeV}$ ) in the knockout region for events where the plug electron is reconstructed in a region of the calorimeter that has a 48-wedge segmentation in $\phi . \ldots \ldots \ldots \ldots \ldots$. . . . . . . . . . . . . . . . . . . . . . . . . . . 
8.4 The energy deposited (in $\mathrm{MeV}$ ) in the knockout region for a plug electron reconstructed in tower number -11 where the plug electron is in the 24 -wedge $\phi$ slice but on the boundary between a 24 and 48 -wedge region. . . . . . . . . 139

8.5 The energy deposited (in $\mathrm{MeV}$ ) in the knockout region for a plug electron reconstructed in tower number -12 where the plug electron is in the 48 -wedge $\phi$ slice but on the boundary between a 24 and 48 -wedge region. . . . . . . . 139

8.6 The energy deposited (in $\mathrm{MeV}$ ) in the knockout region for a plug electron reconstructed in tower number -17 tower where the plug electron is in the 48-wedge $\phi$ slice but on the boundary between a 24 and 48-wedge region. . . . 140

8.7 The energy deposited (in $\mathrm{MeV}$ ) in the knockout region for a plug electron reconstructed in tower number -18 where the plug electron is in the 24 -wedge $\phi$ slice but on the boundary between a 24 and 48 -wedge region. . . . . . . . 140

8.8 Fits to the invariant mass distribution in $\mathrm{CP} \mathrm{Z} \rightarrow e^{+} e^{-}$events for the plug electron in the region (a) $-2.8<\eta<-1.6$ (b) $-1.6<\eta<-1.2$ (c) $1.2<\eta<$ 1.6 and $(\mathrm{d}) 1.6<\eta<2.8 \ldots \ldots \ldots \ldots$. . . . . . . . . . . . . . . . . . . . . .

8.9 The fit to the invariant mass distribution of the $\mathrm{Z}$ boson to obtain an overall

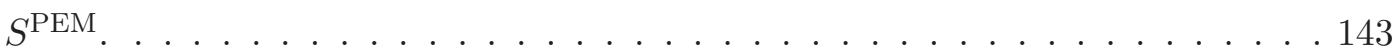

8.10 The plug electron (a) $\eta$ and (b) $\phi$ distributions for data and simulation. . . . . 143

8.11 (a) The $\mathrm{Z}$ boson rapidity distribution in $\mathrm{CP}$ data and simulation events. (b) The plug electron $E_{T}$ distribution. . . . . . . . . . . . . . . 144

8.12 (a) The plug electron ID efficiency as a function of Run Period. (b) The efficiency of $\chi_{3 \times 3}^{2}$ as a function of Run Period. . . . . . . . . . . . . . . . 145

8.13 The plug electron ID efficiency as a function of instantaneous luminosity. . . . 145

8.14 The plug electron ID efficiency as a function of $U_{\|} \ldots \ldots \ldots \ldots$

8.15 The $\mathrm{Z}$ boson $p_{\mathrm{T}}$ distribution simulation and data for $\mathrm{CP} \mathrm{Z} \rightarrow e^{+} e^{-}$events. . . 147

8.16 Underlying energy in a pseudo-cluster in a region orthogonal in $\phi$ but at the same $\eta$ as the plug electron in $\mathrm{CP} \mathrm{Z} \rightarrow e^{+} e^{-}$events. . . . . . . . . . . . 148

8.17 The percentage of events with no recoil energy in the pseudo-cluster as a function of tower number. 
8.18 The calorimeter isolation fraction distribution for the 'anti-electron' sample and the invariant mass of the Z boson in the 'anti-electron' sample. . . . . . . . 151

8.19 Fit to the calorimeter isolation fraction in the data to determine the fraction of QCD background in $\mathrm{CP} \mathrm{Z} \rightarrow e^{+} e^{-}$events. . . . . . . . . . . . . 152

8.20 The shape of the recoil distribution in background events contributing to the CP sample. . . . . . . . . . . . . . . . . . . . 153

8.21 The shape of the $U_{1}$ distribution in background events contributing to the $\mathrm{CP}$ sample. . . . . . . . . . . . . . . . . . . . 153

8.22 The shape of the $U_{2}$ distribution in background events contributing to the CP sample. . . . . . . . . . . . . . . . . . . . . 154

8.23 $\Sigma E_{\mathrm{T}}$ distribution in $\mathrm{CP} \mathrm{Z} \rightarrow e^{+} e^{-}$events compared to the simulation using the best fit $\Sigma E_{\mathrm{T}}$ parameters. . . . . . . . . . . . . . 155

8.24 The dependence of the mean and standard deviation of the $\Sigma E_{\mathrm{T}}$ on luminosity and boson $p_{\mathrm{T}}$ in $\mathrm{CP} \mathrm{Z} \rightarrow e^{+} e^{-}$events. These distributions are used in the fit to obtain the $\Sigma E_{\mathrm{T}}$ parameters. . . . . . . . . . . . . . 156

8.25 The variation of the $U_{1}$ response with boson $p_{\mathrm{T}}$ in $\mathrm{CP} \mathrm{Z} \rightarrow e^{+} e^{-}$events. . . . 156

8.26 The variation of the resolution of $U_{1}$ with boson $p_{\mathrm{T}}$ in $\mathrm{CP} \mathrm{Z} \rightarrow e^{+} e^{-}$events. $\quad 157$

8.27 The variation of the resolution of $U_{2}$ with boson $p_{\mathrm{T}}$ in $\mathrm{CP} \mathrm{Z} \rightarrow e^{+} e^{-}$events. $\quad 157$

8.28 Recoil distributions of (a) $U_{1}$, (b) $U_{2}$ and (c) $U$ in $\mathrm{CP} \mathrm{Z} \rightarrow e^{+} e^{-}$events compared to the best fit simulation. . . . . . . . . . . . . . 158

9.1 Comparison between the shape of the $M_{T}$ distribution in $\mathrm{W} \rightarrow \tau \nu, \mathrm{Z} \rightarrow e^{+} e^{-}$ and $\mathrm{Z} \rightarrow \tau^{+} \tau^{-}$background events in the $\mathrm{W} \rightarrow e \nu$ candidate sample. . . . . . 162

9.2 The diffractive production of a $\mathrm{W}$ boson at (a) LO from a quark interaction in the pomeron $(\mathrm{P})$ and $(\mathrm{b})$ at NLO from a gluon. . . . . . . . . . . 163

9.3 The invariant mass distribution in $\mathrm{Z} \rightarrow e^{+} e^{-}$sample for electrons with same sign charge. . . . . . . . . . . . . . . . . . 165

9.4 The calorimeter isolation fraction distribution in the 'anti-electron' sample and the invariant mass of the $\mathrm{Z}$ boson before and after correcting for electroweak

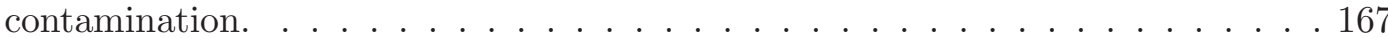


9.5 (a) Result of the fit to the calorimeter isolation fraction distribution to obtain the normalisation of QCD background. (b) The variation of the $\chi^{2}$ of the fit as a function of the amount of QCD background. . . . . . . . . . . 167

9.6 The $E_{T}$ distribution in the 'anti-electron' sample before and after correcting for contamination from electroweak backgrounds. . . . . . . . . . . . . . . . 171

9.7 The fraction of QCD background obtained for different 'anti-electron' selection criteria. The points 1,9 and 10 are those used in the final QCD background estimate. . . . . . . . . . . . . . . . . . . . 172

9.8 (a) Result of the $E_{T}$ fit to data using a simulation $\mathbb{H}_{T}$ distribution comprising of the signal, QCD background and electroweak backgrounds. (b) The variation of the $\chi^{2}$ of the fit as a function of the amount of QCD background. . . . . . . 172

10.1 Distribution of $M_{T}$ for (a) $\mathrm{W} \rightarrow e \nu$ and (b) $\mathrm{W} \rightarrow \mu \nu$ events compared to the simulation for the $\mathrm{W}$ width analysis. . . . . . . . . . . . . . 175

10.2 The width of the $\mathrm{W}$ boson as measured by the LEP and Tevatron experiments. The CDF (Run II) result is the measurement described in this thesis. The Tevatron and world average values include this result. . . . . . . . . . 176

10.3 (a) Distribution of $M_{T}$ for $\mathrm{W} \rightarrow e \nu$ events compared to the simulation using the best fit recoil model parameters and (b) the $\chi$ plot for the comparison between data and simulation. . . . . . . . . . . . . . . . . . . 179

10.4 (a) Distribution of $M_{T}$ for $\mathrm{W} \rightarrow \mu \nu$ events compared to the simulation using the best fit recoil model parameters and (b) the $\chi$ plot for the comparison between data and simulation. . . . . . . . . . . . . . . . . . . 179 


\section{List of Tables}

2.1 Resolutions of the calorimeter sub-systems. . . . . . . . . . . . . . . . . 24

4.1 Selection criteria for electrons in the mass and width analyses. . . . . . . . . 47

4.2 Selection criteria for 'loose' muons in the mass and the width analyses. . . . . . 49

4.3 Additional selection criteria for 'tight' muons in the mass and the width analyses. 49

4.4 Event cuts for the $\mathrm{W} \rightarrow l \nu$ sample in the $\mathrm{W}$ mass and width analyses. . . . . . 50

4.5 Event cuts for the $\mathrm{Z} \rightarrow l^{+} l^{-}$sample in the $\mathrm{W}$ mass and width analyses. . . . . 51

4.6 Event yields for the event samples used in the W mass and width analyses. . . 51

5.1 Systematic uncertainties on $\Gamma_{W}$ due to $1 \sigma$ uncertainty in the CTEQ and MRST

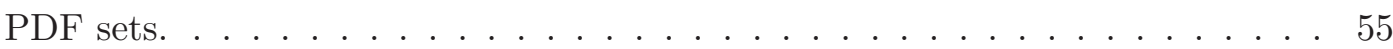

5.2 Systematic uncertainty on $p_{T}^{W}$ from various contributions. . . . . . . . . . 60

5.3 Systematic uncertainties on $\Gamma_{W}$ due to QED for the electron and muon decay channels. . . . . . . . . . . . . . . . . . . . 62

5.4 The shift in $\Gamma_{W}$ due to non-resonant electroweak corrections for the electron and muon decay channels . . . . . . . . . . . . . . . . . 62

6.1 Systematic uncertainties on the $\mathrm{W}$ width in $\mathrm{W} \rightarrow e \nu$ events from the simulation of energy loss by electrons. . . . . . . . . . . . . . . . . . . . . . 69

6.2 Systematic uncertainties on the $\mathrm{W}$ width in $\mathrm{W} \rightarrow \mu \nu$ events from the COT momentum scale, resolution and the non-Gaussian fraction. . . . . . . . . . . 73

6.3 Systematic uncertainties on the $\mathrm{W}$ width in $\mathrm{W} \rightarrow e \nu$ events from the uncertainty on the calorimeter scale and resolution. . . . . . . . . . . . . 80 
6.4 Summary of the systematic uncertainties associated with the simulation of leptons. . . . . . . . . . . . . . . . . . . 82

7.1 The shifts in $(x, y)$ for the East and West halves of the plug calorimeter for three run ranges. . . . . . . . . . . . . . . . . . . . 91

7.2 The best fit $\Sigma E_{\mathrm{T}}$ parameters obtained by fitting $\mathrm{Z} \rightarrow e^{+} e^{-}$and $\mathrm{Z} \rightarrow \mu^{+} \mu^{-}$ events in the $\mathrm{W}$ width analysis. . . . . . . . . . . . . . . . . . 101

7.3 The $\Sigma E_{\mathrm{T}}$ parameters obtained by fitting $\mathrm{Z} \rightarrow e^{+} e^{-}$and $\mathrm{Z} \rightarrow \mu^{+} \mu^{-}$events in the $\mathrm{W}$ mass analysis. . . . . . . . . . . . . . . . . . . . . 104

7.4 The best fit recoil parameters and their statistical uncertainty for $\mathrm{Z} \rightarrow e^{+} e^{-}$ and $\mathrm{Z} \rightarrow \mu^{+} \mu^{-}$events in the $\mathrm{W}$ width analysis. . . . . . . . . . . . . . 109

7.5 The best fit recoil parameters and their statistical uncertainty for $\mathrm{Z} \rightarrow e^{+} e^{-}$ and $\mathrm{Z} \rightarrow \mu^{+} \mu^{-}$events in the $\mathrm{W}$ mass analysis. . . . . . . . . . . . . 110

7.6 Systematic uncertainty on $\Gamma_{W}$ from the recoil in the electron and muon channels for the $\mathrm{W}$ width analysis. . . . . . . . . . . . . . . 122

7.7 Systematic uncertainty on $M_{W}$ from the recoil in the electron and muon channels for the $\mathrm{W}$ mass analysis. . . . . . . . . . . . . . . . . . . 122

7.8 Systematic uncertainty on $M_{W}$ from the discrepancy in the $U$ distribution, the discrepancy in the low $\Sigma E_{\mathrm{T}}$ distribution, the discrepancy in the $\sigma\left(U_{2}\right)$ vs. $p_{\mathrm{T}}$ distribution and tuning the recoil only on $\mathrm{CC}$ events. . . . . . . . . . . . . 122

8.1 Selection criteria for plug electrons in $\mathrm{CP} \mathrm{Z} \rightarrow e^{+} e^{-}$events. . . . . . . . . . 135

8.2 The number of towers in the knockout region depending on the tower of the plug electron. . . . . . . . . . . . . . . . . . . 137

8.3 The PEM scale and resolution obtained by fitting to the invariant mass of the Z boson in four $\eta$ regions. . . . . . . . . . . . . . . . . . . 143

8.4 The background processes contributing to $\mathrm{CP} \mathrm{Z} \rightarrow e^{+} e^{-}$events and their relevant fractions. . . . . . . . . . . . . . . . . . . . . . 153

8.5 The recoil parameters obtained from fits to the recoil in $\mathrm{CP}$ and $\mathrm{CC} \mathrm{Z} \rightarrow e^{+} e^{-}$ events. . . . . . . . . . . . . . . . . . . . . . 159 
10.1 Summary of the systematic and statistical uncertainties, in $\mathrm{MeV}$, on the width of the $\mathrm{W}$ boson in the $\mathrm{W} \rightarrow e \nu$ and $\mathrm{W} \rightarrow \mu \nu$ decay channels. . . . . . . . . 175

10.2 Summary of the systematic uncertainties, in $\mathrm{MeV}$, on $M_{W}$ from the recoil, including the uncertainty from the $\mathrm{Z}$ fit statistics, the discrepancy in the $U$ distribution and the $\sigma\left(U_{2}\right)$ distribution, the discrepancy in the low $\Sigma E_{\mathrm{T}}$ distribution and tuning the recoil only on CC events. . . . . . . . . . . . . 178

10.3 Summary of some of the systematic uncertainties on $M_{W}$ in the electron channel as measured in the previous $\mathrm{W}$ mass measurement and the projected systematic uncertainties for the current measurement. . . . . . . . . . . . . 178 


\section{Chapter 1}

\section{The Standard Model}

The Standard Model of particle physics is a theoretical framework describing the elementary particles and the interactions between them. It consists of a set of gauge field theories that couple the three generations of particles that constitute matter to a different class of particles that mediate the strong, electromagnetic and weak interactions.

The model was developed in the 1970s and since then it has been exceptionally successful in explaining experimental observations and predicting the outcome of a large number of experiments. The heaviest particle predicted by the Standard Model, the top quark, was discovered in proton antiproton collisions at the CDF [1] and DØ [2] detectors at the Tevatron and the $\mathrm{W}$ and $\mathrm{Z}$ bosons predicted by the theory that unified the electromagnetic and weak forces, the electroweak theory, were discovered in the early 1980s at the UA1 [3] and UA2 [4] experiments at CERN.

The Standard Model has been validated to an extraordinary level of precision with more precise experimental measurements testing the Standard Model quantities at the level of radiative corrections. The measurements of the mass and width of the $\mathrm{W}$ boson presented in this thesis represent a continuing effort towards constraining the theory.

\subsection{Particles}

The particles in the Standard Model can be divided into two classes; fermions and bosons. Fermions are spin $\frac{1}{2}$ particles obeying Fermi-Dirac statistics and can be further sub-divided into leptons and quarks. Bosons have integer spin and obey Bose-Einstein statistics. 


\section{- Quarks}

The most important property of quarks that distinguishes them from leptons is that they are not found isolated in nature. They exist in composite states called hadrons, which consist of 2 or 3 quarks bound together by the strong force. There are six different types of quarks with varying mass and charge, called up (u), down (d), charm (c), strange (s), bottom (b) and top (t). They have spin $\frac{1}{2}$ and unlike the leptons, fractional charge. The $\mathrm{u}, \mathrm{c}$ and $\mathrm{t}$ quarks have a charge of $+\frac{2}{3}$ and the $\mathrm{d}, \mathrm{s}$ and $\mathrm{b}$ quarks have a charge of $-\frac{1}{3}$. Their antiparticles also have fractional charges but of opposite sign. They are assigned a quantum number called 'colour charge' which enables them to interact via the strong interaction. They are also affected by the electromagnetic and weak interactions.

\section{- Leptons}

Leptons are spin $\frac{1}{2}$ pointlike particles. There are three known flavours of leptons; electron, muon and tau. Each flavour is represented by a weak doublet, which consists of a massive charged particle and a nearly massless neutral particle called the neutrino. All six leptons have corresponding antiparticles. Leptons couple to the electroweak gauge bosons and take part in the weak interaction with the charged leptons also taking part in the electromagnetic interaction. They do not possess colour charge and are thus unaffected by the strong force.

\section{- Vector Bosons}

Vector bosons mediate interactions between quarks and leptons with each gauge boson associated with a fundamental interaction. The electromagnetic interaction between charged particles is mediated by the massless photon, the weak interaction by the massive $\mathrm{W}^{ \pm}$and $\mathrm{Z}$ bosons and the strong force by 8 massless gluons. 


\subsection{Electromagnetic Interaction}

The theory describing the interaction between particles possessing electric charge is known as QED (Quantum Electro Dynamics). It is an Abelian gauge field theory obeying the $\mathrm{U}(1)$ symmetry group. The Lagrangian density, $\mathcal{L}$, for a free Dirac fermion field $\psi$ with mass $m$ is given by

$$
\mathcal{L}=\bar{\psi}\left(i \gamma^{\mu} \partial_{\mu}-m\right) \psi
$$

The QED Lagrangian is required to be invariant under a local phase transformation. This is achieved by introducing a vector gauge field $A_{\mu}$ such that the covariant derivative $D_{\mu}$ has the form

$$
D_{\mu}=\partial_{\mu}+i e Q A_{\mu}
$$

where $e Q$ is the charge of the fermion and the gauge field $A_{\mu}$ can be identified as the photon field. The QED Lagrangian is written as

$$
\mathcal{L}=\bar{\psi}\left(i \gamma^{\mu} D_{\mu}-m\right) \psi+\frac{1}{4} F_{\mu \nu} F^{\mu \nu}
$$

where the last term denotes a kinetic term for the photon field formed by defining a field strength tensor $F_{\mu \nu}$ as

$$
F_{\mu \nu}=\partial_{\mu} A_{\nu}-\partial_{\nu} A_{\mu}
$$

There is no mass term for the photon field since a term of the form $m^{2} A_{\mu} A^{\mu}$ would violate gauge symmetry. This is therefore consistent with what is observed in nature, i.e. the photon is massless.

\subsection{Strong Interaction}

The strong interaction is described by a non-Abelian gauge theory known as QCD (Quantum Chromo Dynamics) which is based on the symmetry of the gauge group SU(3). Requiring the Standard Model Lagrangian to be invariant under local gauge transformations 
introduces 8 massless gluons which correspond to the 8 generators of the symmetry group $\mathrm{SU}(3)$. Quarks and gluons are collectively known as partons and are found to have an internal quantum degree of freedom known as colour comprising of three states; blue, green and red. The exchange of this colour charge between gluons allows them to interact with one another. The QCD Lagrangian for a quark field $q$ is

$$
\mathcal{L}=\bar{q}\left(i \gamma^{\mu} D_{\mu}-m\right) q+\frac{1}{4} F_{\mu \nu} F^{\mu \nu}
$$

with the covariant derivative given by

$$
D_{\mu}=\partial_{\mu}+i g_{s} T_{a} A_{\mu}^{a}
$$

where $T_{a}$ are the set of $8 \mathrm{SU}(3)$ generators with index $a$ going from 1 to 8 and $g_{s}$ is a coupling constant characterising the strength of the strong interaction. The last term in the Lagrangian represents the kinetic term for the gluon fields and the field strength tensor is defined as

$$
F_{\mu \nu}^{a}=\partial_{\mu} A_{\nu}^{a}-\partial_{\nu} A_{\mu}^{a}-g_{s} f_{a b c} A_{\mu}^{b} A_{\nu}^{c}
$$

where $f_{a b c}$ are the structure functions of the SU(3) group. Again, as in the case of QED, there is no gauge invariant mass term in the Lagrangian, resulting in the gluons being massless. The last term in the gluon field strength tensor denotes the self interactions of the gluon field. This can be compared to the expression for the photon field tensor in Equation 1.4 where the absence of such a term shows the Abelian nature of QED. Also in contrast to QED, the coupling strength of QCD decreases as the energy scale increases. This leads to a number of interesting features which account for the properties and behaviour of quarks and their interactions, such as confinement and asymptotic freedom.

Confinement : Quarks are not found isolated in nature, they are only observed in bound colour singlet states of hadrons that have integer electric charge and zero colour charge. If one tries to pull a quark out of a hadron, the force between this quark and gluons increases 
until there is sufficient energy to produce a quark-antiquark pair from the vacuum. This then combines with the other quarks to produce hadrons. It is not possible therefore to isolate a single quark from a hadron. This property is called confinement.

Asymptotic freedom : On the other hand, pushing quarks together inside a hadron decreases the distance between them and decreases the force between them such that they can be thought to behave like free particles. This property is known as asymptotic freedom.

\subsection{Electroweak Interaction}

In the 1960s Glashow [5], Salam [6] and Weinberg [7] postulated the theoretical unification of the electromagnetic and weak forces into a single electroweak theory. The weak interaction on its own is described by the gauge group $\mathrm{SU}(2)_{L}(L$ denotes that only left-handed states are involved) and the electromagnetic interaction is described by $\mathrm{U}(1)_{Q}$, where $Q$ represents electromagnetic charge. To unify the two interactions a new quantum number is introduced, the weak hypercharge, $Y$, which is related to $Q$ and the third component of weak isospin, $T_{3}$ in the following way

$$
Q=T_{3}+\frac{Y}{2}
$$

The leptons are characterised as left-handed isospin doublets

$$
L_{e}=\left(\begin{array}{c}
\nu_{e} \\
e^{-}
\end{array}\right)_{L}, L_{\mu}=\left(\begin{array}{c}
\nu_{\mu} \\
\mu^{-}
\end{array}\right)_{L}, L_{\tau}=\left(\begin{array}{c}
\nu_{\tau} \\
\tau^{-}
\end{array}\right)_{L}
$$

and right-handed isospin singlets

$$
R_{e, \mu, \tau}=e_{R}, \mu_{R}, \tau_{R}
$$

The quarks are similarly characterised as left-handed isospin doublets

$$
L_{u d^{\prime}}=\left(\begin{array}{c}
u \\
d^{\prime}
\end{array}\right)_{L}, L_{c s^{\prime}}=\left(\begin{array}{c}
c \\
s^{\prime}
\end{array}\right)_{L}, L_{t b^{\prime}}=\left(\begin{array}{c}
t \\
b^{\prime}
\end{array}\right)_{L}
$$


and right-handed isospin singlets

$$
R_{u}=u_{R}, c_{R}, t_{R} \text { and } R_{d}=d_{R}, s_{R}, b_{R}
$$

The weak eigenstates of the quark doublets $\left(d^{\prime}, s^{\prime}, b^{\prime}\right)$ are mixtures of the mass eigenstates

$$
\left(\begin{array}{c}
d^{\prime} \\
s^{\prime} \\
b^{\prime}
\end{array}\right)=\left(\begin{array}{lll}
V_{u d} & V_{u s} & V_{u b} \\
V_{c d} & V_{c s} & V_{c b} \\
V_{t d} & V_{t s} & V_{t b}
\end{array}\right)\left(\begin{array}{c}
d \\
s \\
b
\end{array}\right)
$$

where the $3 \times 3$ Cabbibo-Kobayashi-Maskawa [9] [10] matrix represents quark mixing.

The $\mathrm{SU}(2)_{L}$ group has $2^{2}-1=3$ generators which give the gauge fields $W_{\mu}^{1}, W_{\mu}^{2}$ and $W_{\mu}^{3}$ and the $\mathrm{U}(1)_{Y}$ group gives the gauge field $B_{\mu}$. The covariant derivative of the electroweak Lagrangian is given by

$$
D_{\mu}=\partial_{\mu}-i g T_{i} W_{\mu}^{i}-i g^{\prime} \frac{Y}{2} B_{\mu}
$$

where $T_{i}$ are traceless Hermitian generators of $\mathrm{SU}(2)$ with $i$ representing a sum over all the generators and the coupling strengths of the electromagnetic and weak interactions is denoted by $g$ and $g^{\prime}$ respectively. The physical gauge bosons $W_{\mu}^{ \pm}$are superpositions of the $\mathrm{SU}(2)$ gauge bosons, $W_{\mu}^{1}$ and $W_{\mu}^{2}$, so

$$
W_{\mu}^{ \pm}=\left(W_{\mu}^{1} \pm i W_{\mu}^{2}\right) / \sqrt{2}
$$

The $W_{\mu}^{3}$ and $B_{\mu}$ fields also mix, like the $W_{\mu}^{1}$ and $W_{\mu}^{2}$ and the physical gauge bosons can be defined as superpositions of these fields in the following way

$$
\begin{aligned}
& Z_{\mu}=\cos \theta_{W} W_{\mu}^{3}+\sin \theta_{W} B_{\mu} \\
& A_{\mu}=\sin \theta_{W} W_{\mu}^{3}+\cos \theta_{W} B_{\mu}
\end{aligned}
$$

where the weak mixing angle, $\theta_{W}$ is defined by

$$
\tan \theta_{W}=\frac{g^{\prime}}{g}
$$


The fact that the neutral gauge bosons, the $\mathrm{Z}$ and the photon, are linear combinations of a gauge boson from each of $\mathrm{SU}(2)$ and $\mathrm{U}(1)$ demonstrates the unification of $\mathrm{SU}(2)$ and $\mathrm{U}(1)$. The gauge invariant Lagrangian describing electroweak interactions is

$$
\mathcal{L}=\bar{L} i \gamma^{\mu} D_{\mu} L+\bar{R} i \gamma^{\mu} D_{\mu} R+\frac{1}{4} f_{\mu \nu} f^{\mu \nu}+\frac{1}{4} F_{\mu \nu} F^{\mu \nu}
$$

where the field strength tensor for the gauge fields $W_{\mu}^{a}$ is given by

$$
F_{\mu \nu}^{a}=\partial_{\mu} W_{\nu}^{a}-\partial_{\nu} W_{\mu}^{a}+i g \epsilon_{a b c} W_{\mu}^{b} W_{\nu}^{c}
$$

and the field strength tensor for the gauge field $B_{\mu}$ is given by

$$
f_{\mu \nu}=\partial_{\mu} B_{\nu}-\partial_{\nu} B_{\mu}
$$

The electroweak Lagrangian does not contain mass terms for the gauge bosons since such terms would violate local gauge invariance. In a symmetric gauge theory, therefore, the gauge bosons must be massless. This is exactly what is required for QED (massless photon) and QCD (massless gluons). However, for the weak interaction the symmetry must somehow be broken since the carriers of the weak interaction (the $\mathrm{W}$ and $\mathrm{Z}$ bosons) are known to be massive. Spontaneous symmetry breaking is a way to break the symmetry of a theory whilst preserving local gauge invariance and keeping the theory renormalisable. The Lagrangian remains invariant under the symmetry transformation, however the ground state of the symmetry is not invariant. Masses are generated via the Higgs mechanism [8] which involves introducing a scalar Higgs field in order to break the symmetry of the group spontaneously. A scalar potential of the form

$$
V_{\phi}=-\mu^{2} \phi^{\dagger} \phi+\lambda\left(\phi^{\dagger} \phi\right)^{2}
$$

is introduced, where $\lambda, \mu$ are constants and the doublet for a complex scalar field is given by

$$
\phi=\left(\begin{array}{c}
\phi^{+} \\
\phi^{0}
\end{array}\right)
$$


For $\lambda>0$, if $\mu^{2}<0$ the potential $V(\phi)$ has a minimum at $\phi=0$. For the case where $\mu^{2}>0$, the potential no longer has a minimum at $\phi=0$ but a maximum, the minimum occurs if $\phi^{\dagger} \phi=-\mu^{2} / 2 \lambda \equiv v^{2} / 2$ where $v$ is the vacuum expectation value. Expanding the Higgs field about the minimum results in the $\mathrm{W}^{ \pm}$and $\mathrm{Z}$ bosons acquiring a mass

$$
M_{W}=\frac{v g}{2} \text { and } M_{Z}=\frac{v \sqrt{g^{2}+g^{\prime 2}}}{2} \text {. }
$$

The spontaneous symmetry breaking of the electroweak theory therefore results in one massless gauge boson, the photon and three massive gauge bosons, $W^{ \pm}$and $Z$ where the masses are generated via the Higgs mechanism. Indeed, the observation of the $W^{ \pm}$at the $S p \bar{p} S$ collider at CERN in 1982 was a great vindication of the electroweak theory. The particle responsible for giving them masses, the Higgs, has yet to be discovered.

\subsection{Beyond the Standard Model}

Over the last few decades, significant upgrades in accelerators have enabled us to collide particles at successively higher energies, resulting in the discovery of the top quark at the Tevatron and the $\mathrm{W}$ and $\mathrm{Z}$ bosons at CERN, while precision measurements, such as the $\mathrm{W}$ mass and width measurements presented in this thesis, have enabled stringent constraints to be placed on Standard Model quantities.

Through this process, the Standard Model has been tried, tested and constrained and it has done remarkably well in explaining a large fraction of experimental observations, with the notable exception being neutrino masses. The observation of neutrino oscillations [11] contradicts the Standard Model prediction of massless neutrinos. This section will briefly mention some of the reasons why the Standard Model is not thought to be a complete theory.

\section{- Gravity}

One of the major shortcomings of the Standard Model is that it does not explain 
gravity. The Standard Model is therefore thought to be valid up to the Planck scale, $m_{\text {Planck }} \sim 10^{19} \mathrm{GeV}^{1}$, the scale at which gravity is expected to display quantum behaviour and hence become important. A theory that claims to describe everything would need to include gravity.

\section{- Hierarchy Problem}

All particles predicted by the Standard Model have been observed in experiments, except the Higgs boson. The mass of the Higgs boson receives quadratically divergent corrections from loops of virtual particles. Since the Standard Model is considered to be an effective theory valid up to $m_{\text {Planck }}$, these contributions could be as large as the Planck scale. Some very delicate fine tuning is required to cancel contributions of the order of $m_{\text {Planck }}$ to bring the Higgs mass down to the electroweak scale, of $\mathcal{O}((100) \mathrm{GeV})$. This is known as the hierarchy problem.

- Dark matter

Evidence from cosmological experiments suggests that only a very small percentage $(\sim 4 \%)$ of the universe is 'visible', i.e. it emits electromagnetic radiation. Almost $22 \%$ of the universe is composed of what is known as dark matter, matter that emits no radiation. Its presence was inferred by studying the rotational velocities of galaxies. The dark matter candidate is thought to be a stable, neutral and massive particle.

\section{- GUT}

The idea that the three forces contained in the Standard Model are simply manifestations of a single force is called the Grand Unified Theory (GUT). It predicts that at some very high energy the three forces merge into a single force and the coupling

\footnotetext{
${ }^{1}$ Throughout this thesis, the relation $\hbar=c=1$ is used. Therefore, mass, momentum and energy are all expressed in $\mathrm{eV}$.
} 
constants intersect. Running the coupling constants to higher energies shows that although the couplings come close, they do not intersect.

A number of theories have been proposed to address these issues, one of the most popular being Supersymmetry [12]. Supersymmetry or SUSY, is a theory which predicts a Supersymmetric fermionic (bosonic) partner for every Standard Model boson (fermion). Supersymmetry is thought to be a broken symmetry and as a result the superpartners are heavier than the known elementary particles and have not yet been observed experimentally. SUSY has a number of interesting features which make it appealing. The spectra of particles predicted by SUSY enter as virtual loop corrections into the Higgs mass. These corrections are opposite in sign to those from Standard Model particles and therefore solve the hierarchy problem by reducing the number of quadratically divergent terms in the Higgs mass such that the Higgs mass is of the order of electroweak scale. In addition, the lightest particle from the SUSY particle spectrum is stable and a good candidate for cold dark matter. Introducing SUSY particles into the theory also changes the way the coupling constants vary with energy. With a certain choice of masses for SUSY particles the coupling constants can be made to intersect at high energy.

One of the goals of the Large Hadron Collider (LHC) built at CERN is to look for physics beyond the Standard Model by searching for new particles such as those proposed by SUSY. 


\section{Chapter 2}

\section{The Tevatron and CDF}

The data used to perform the $\mathrm{W}$ mass and width measurements was collected by the Collider Detector at Fermilab (CDF), a multi-purpose detector used to study protonantiproton collisions at the Tevatron accelerator. This chapter describes the accelerator and the detector, focusing on components of the detector that are most relevant to the analyses presented in this thesis.

\subsection{The Tevatron}

The Tevatron is the world's highest energy particle accelerator located at the Fermi National Accelerator Laboratory (Fermilab), Illinois. It brings together beams of protons and antiprotons travelling at almost the speed of light and collides them head-on at a centre-of-mass energy of $1.96 \mathrm{TeV}$. A chain of accelerators are employed in a series of steps culminating in the collision of proton-antiproton beams in the Tevatron with each beam attaining an energy of $980 \mathrm{GeV}$. A schematic diagram of the stages of production and acceleration is shown in Figure 2.1.

\section{Proton Production and Acceleration}

- $H^{-}$ions, which are just hydrogen atoms albeit with an extra electron are accelerated by the Cockroft-Walton accelerator to $750 \mathrm{keV}$. 
- These are then injected into the linac, a linear accelerator about $150 \mathrm{~m}$ in length, which accelerates them to $400 \mathrm{MeV}$.

- The $H^{-}$ions are subsequently fed into the Booster, a proton synchrotron with a series of magnets arranged around a $75 \mathrm{~m}$ radius circle. Here, the $\mathrm{H}^{-}$ions are stripped of their two electrons by passing them through a carbon foil. The resultant protons are accelerated to $8 \mathrm{GeV}$.

- These $8 \mathrm{GeV}$ protons are passed to the Main Injector, a multi-purpose synchrotron seven times larger than the Booster with 18 accelerating cavities. It can accelerate the protons to two different energies, depending on their subsequent use. If the protons are to be used to produce antiprotons, they are accelerated to $120 \mathrm{GeV}$, if they are to be injected into the Tevatron, their maximum energy is $150 \mathrm{GeV}$.

Antiprotons, not being readily available, must be produced, stored until sufficient amounts are accumulated, then accelerated and fed into the Main Injector.

\section{Antiproton Production and Acceleration}

The production of antiprotons involves firing the protons from the Main Injector at a nickel target. The collision produces a spectra of secondary particles and antiprotons are selected from this shower using a bending magnet which acts as a charge-mass spectrometer. They are then cooled, formed into a beam of antiprotons with a uniform $8 \mathrm{GeV}$ energy and stored in the Recycler until a sufficient quantity, or a 'store' has been collected. These antiprotons are subsequently sent to the Main Injector where they circulate in the opposite direction to the protons and are accelerated to $150 \mathrm{GeV}$.

\section{Tevatron}


The proton-antiproton beams from the Main Injector, each consisting of 36 bunches of protons and antiprotons are passed into the Tevatron, the largest of the Fermilab accelerators, where the final acceleration and collision of the protons and antiprotons takes place. The Tevatron is a $1 \mathrm{~km}$ radius synchrotron ring which employs superconducting magnets and eight accelerating cavities. It accelerates the protonantiproton bunches in opposite directions from $150 \mathrm{GeV}$ to $980 \mathrm{GeV}$ and brings them to collision at 2 predetermined points on the ring, B0 and D0, the locations of the $\mathrm{CDF}$ and DØ detectors, respectively. The centre-of-mass energy of the collision is $\sqrt{s}=1.96 \mathrm{TeV}$ and the time between bunch crossings is $396 \mathrm{~ns}$.

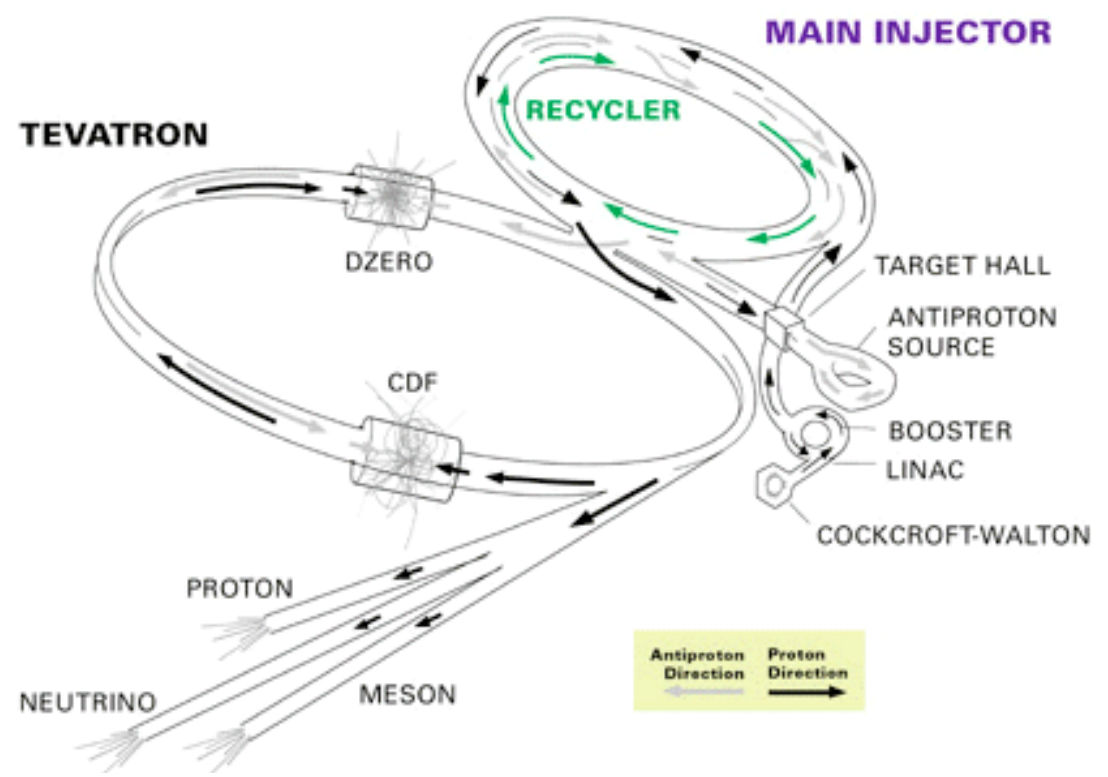

Figure 2.1: The various stages of production and acceleration of protons and antiprotons.

\subsection{Tevatron performance and luminosity}

The performance of the collider is quantified by the instantaneous luminosity which is defined as

$$
L=\frac{n f N_{p} N_{\bar{p}}}{4 \pi \sigma^{2}}
$$


where $N_{p}\left(N_{\bar{p}}\right)$ is the number of $p(\bar{p})$ per bunch, $n$ is the number of bunches, $f$ is the collision frequency and $\sigma^{2}$ is the cross sectional area of the beam. The instantaneous luminosity is at its peak at the beginning of a store and then decreases exponentially with time as particles are lost and the beams lose focus. The peak instantaneous luminosity is shown as a function of time for CDF Run II in Figure 2.2. The increase in peak luminosity over time is the result of improvements in collider operations through more efficient storage and transfer of antiprotons.

The integrated luminosity is a quantitative measure of the amount of data collected over time. The integrated luminosity delivered by the Tevatron over the period of Run II is shown in Figure 2.3. It shows that the total integrated luminosity delivered by the Tevatron has now surpassed $7 \mathrm{fb}^{-1}$. The datasets used in the $\mathrm{W}$ mass and width analyses presented in this thesis represent an integrated luminosity of $2.4 \mathrm{fb}^{-1}$ and 350 $\mathrm{pb}^{-1}$ respectively.

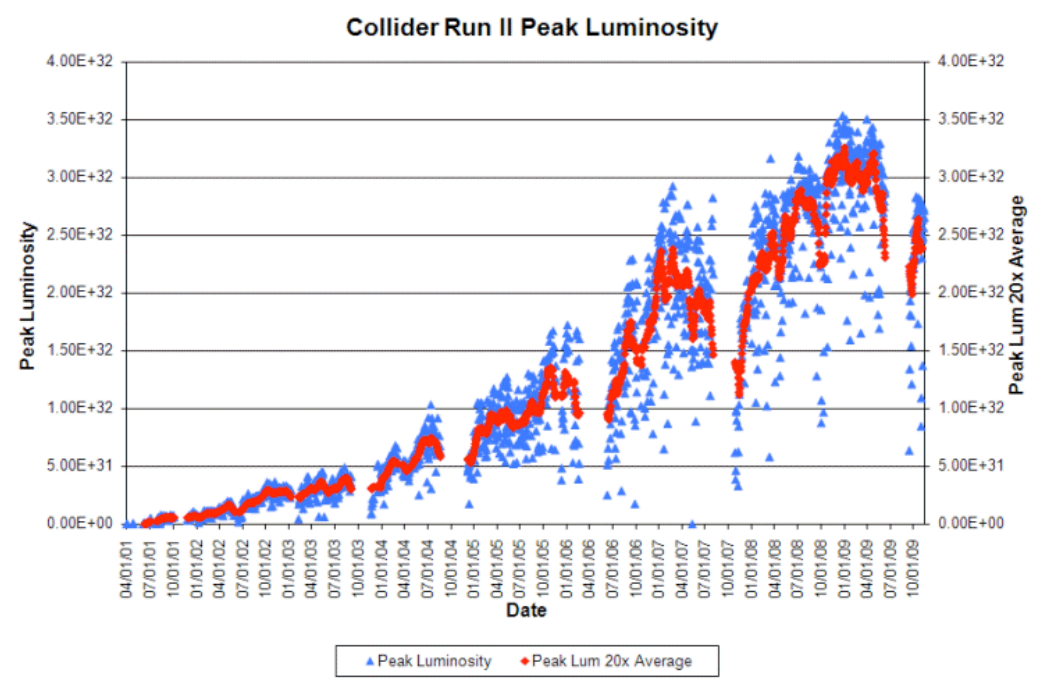

Figure 2.2: The peak luminosity of a store as a function of time for the period of Run II. 


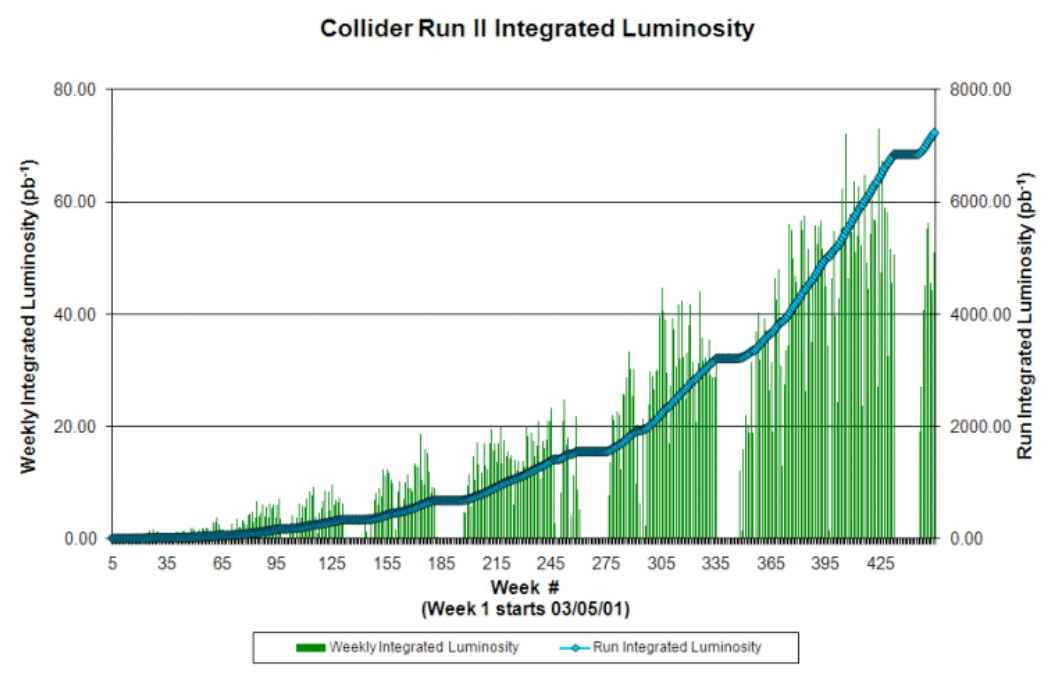

Figure 2.3: The integrated luminosity as a function of time for the period of Run II.

\subsection{The CDF Detector}

The Collider Detector at Fermilab (CDF) [13], shown in Figure 2.4, is a multi-purpose detector designed to study a broad range of interactions and particles produced in the $p \bar{p}$ collision. The proton and antiproton bunches are brought to a focus in the centre of the detector. The resulting particles are identified and their energy and momenta measured by a system of sub-detectors placed in concentric layers around the beam pipe. CDF is a forward-backward and azimuthally symmetric detector reflecting the symmetry of the colliding beams. It comprises of a central barrel region and two end-caps placed on either side of the barrel.

A particle travelling outwards from the point of the proton-antiproton collision first traverses the tracking system which consists of a silicon detector and a drift chamber placed in a magnetic field. Then there is a system of calorimeters, electromagnetic and hadronic, designed to absorb and hence measure the energy of the particle. The outermost detectors are the muon chambers. These sub-detectors are described in more detail in subsequent sections with more emphasis on the components that are relevant to the analyses presented 
in this thesis.

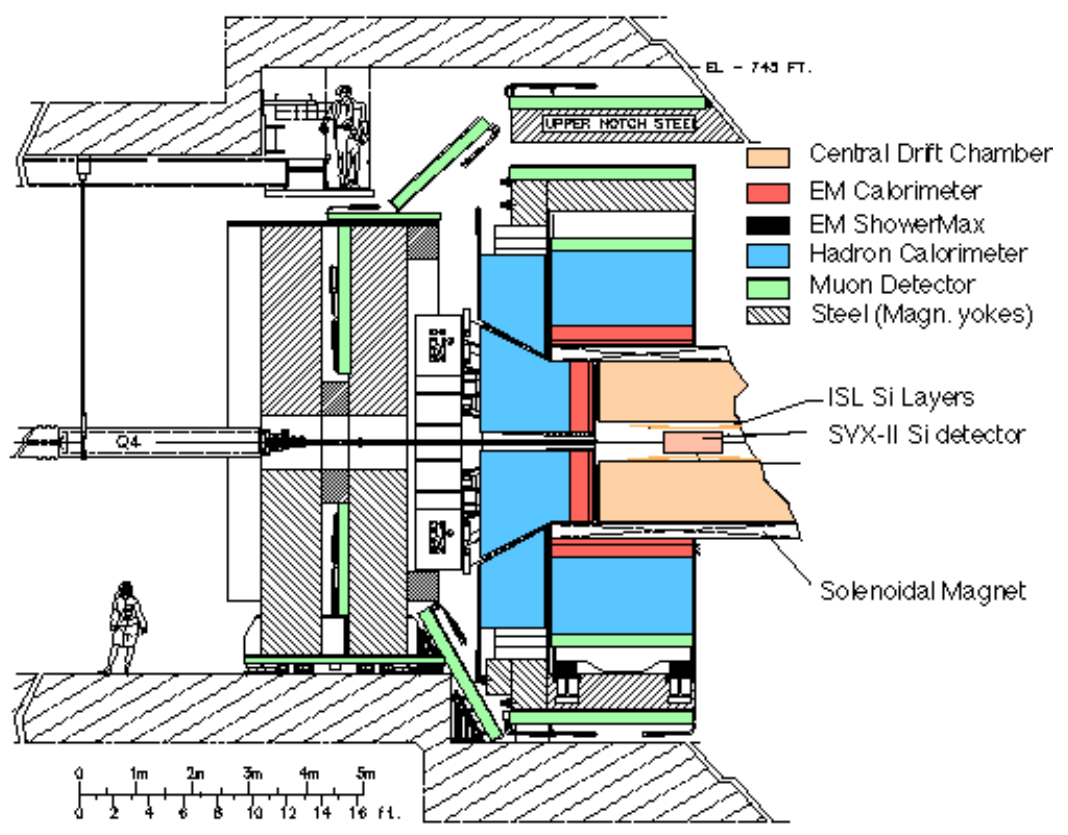

Figure 2.4: An elevation view of one half of the CDF detector.

\subsubsection{CDF Coordinate System}

CDF uses a right-handed coordinate system with an origin at the centre of the detector.

The $z$-axis is oriented along the direction of the beams, it is positive in the direction of the proton beam and negative in the antiproton beam direction. The $y$-axis points vertically upwards from the beam axis and the $x$-axis is in the transverse plane pointing horizontally away from the centre of the detector. The cylindrical symmetry of the detector makes it convenient to introduce a cylindrical coordinate system, where $r$ is the distance from the $z$-axis, the angle $\phi$ is measured in the transverse $(x y)$ plane and the polar angle $\theta$ is measured relative to the $z$-axis. The CDF coordinate system is shown in Figure 2.5. The pseudorapidity is defined as

$$
\eta \equiv-\ln \left(\tan \frac{\theta}{2}\right)
$$


This quantity is useful as it is Lorentz invariant under boosts in the $z$ direction. The components of the detector, in particular the calorimeter system are partitioned in terms of $\eta$ and $\phi$. These coordinates will be referred to in the following sections.

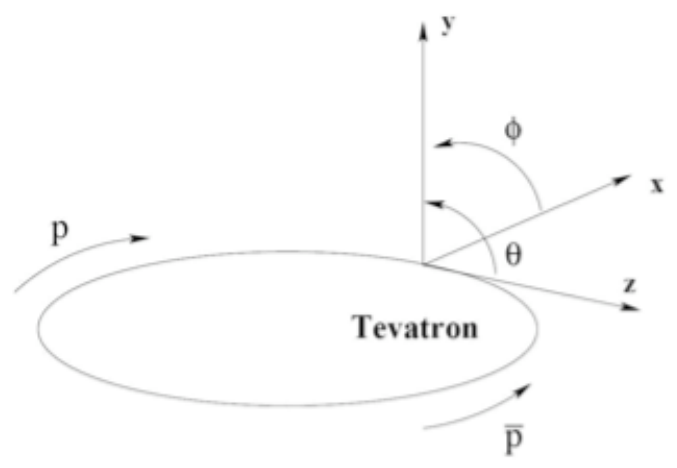

Figure 2.5: The CDF coordinate system.

\subsubsection{Tracking System}

The CDF tracking system is designed to reconstruct the trajectory of charged particles and find vertices associated with the $p \bar{p}$ interaction. It comprises of two sub-detectors placed in a magnetic field of $1.4 \mathrm{~T}$ provided by a superconducting solenoid. The innermost subsystem is a silicon detector which is placed very close to the beam pipe. This is followed by the Central Outer Tracker (COT), a drift chamber which provides coverage over the central region, defined in pseudorapidity as $|\eta| \leq 1$.

\section{Silicon Tracker}

The Silicon Tracker [14] consists of 3 sub-systems designed to provide precise measurement of the particle trajectory close to the beam line. They are placed at increasing radii from the beam pipe with the innermost detector, Layer-00, placed at a radius of $1.35 \mathrm{~cm}$. It comprises of a single sided layer of silicon and provides a measurement of the impact parameter of a particle track. Outside Layer-00 is the SVX (Silicon Vertex Detector) which comprises of 3 barrels placed end to end, each 
$29 \mathrm{~cm}$ long. Each barrel has layers of double sided silicon, with one side providing measurements in the $r-\phi$ plane and the other side providing measurements in the $r-z$ plane. Readout from these is combined to provide 3-D tracking information. Furthest from the beam pipe is the ISL (Intermediate Silicon Layer) which comprises of 3 layers of silicon at varying radii. The inner layer at $20 \mathrm{~cm}$ is in the central region, i.e. $|\eta|<1$ while the outer layers at 22 and $28 \mathrm{~cm}$ respectively are in the region $1<|\eta|<2$, thus providing additional tracking information in the forward region where there is no COT coverage.

\section{The Central Outer Tracker}

The COT [15] is a $3.2 \mathrm{~m}$ long open-cell drift chamber extending from a radius of 40 $\mathrm{cm}$ to $132 \mathrm{~cm}$ from the beam pipe. It is filled with a mixture of argon and ethane in a 50:50 ratio together with a small amount of alcohol. In addition, small measures of oxygen have also been introduced to this mixture after it was observed that a small amount of oxygen reversed the ageing process of the COT, thought to be caused by the build up of polymers on the wires, reducing their gain [18].

The passage of a charged particle through a gas mixture excites and ionises the gas molecules to produce ions and electrons. The electrons drift towards the anode that can be read out to give a precise position measurement and the arrival time of the electrons can be used to calculate a drift time (difference between collision time and time of arrival at the anode). The maximum drift time is required to be less than the time between bunch crossings (396 ns) so that two different bunch crossings can be resolved. In the COT a maximum drift time of $177 \mathrm{~ns}$ is achieved.

The structure of the COT comprises of 8 superlayers, alternating between axial and stereo superlayers, as shown in Figure 2.6. A track with $|\eta|<1$ traverses all 8 superlayers of the COT and are well reconstructed. Axial superlayers have wires 
that are parallel to the $z$-axis and give the $r-\phi$ position of the track and stereo superlayers have wires that are tilted at $\pm 2^{\circ}$ with respect to the $z$-axis, giving the $z$ position of the track. Information from the two is combined to obtain a 3-D reconstruction of the track. Each superlayer is further segmented into 12 layers of wires, each containing sense wires and potential wires. Sense wires are used to collect information about the particle tracks and potential wires are used to configure the electric field in the COT.

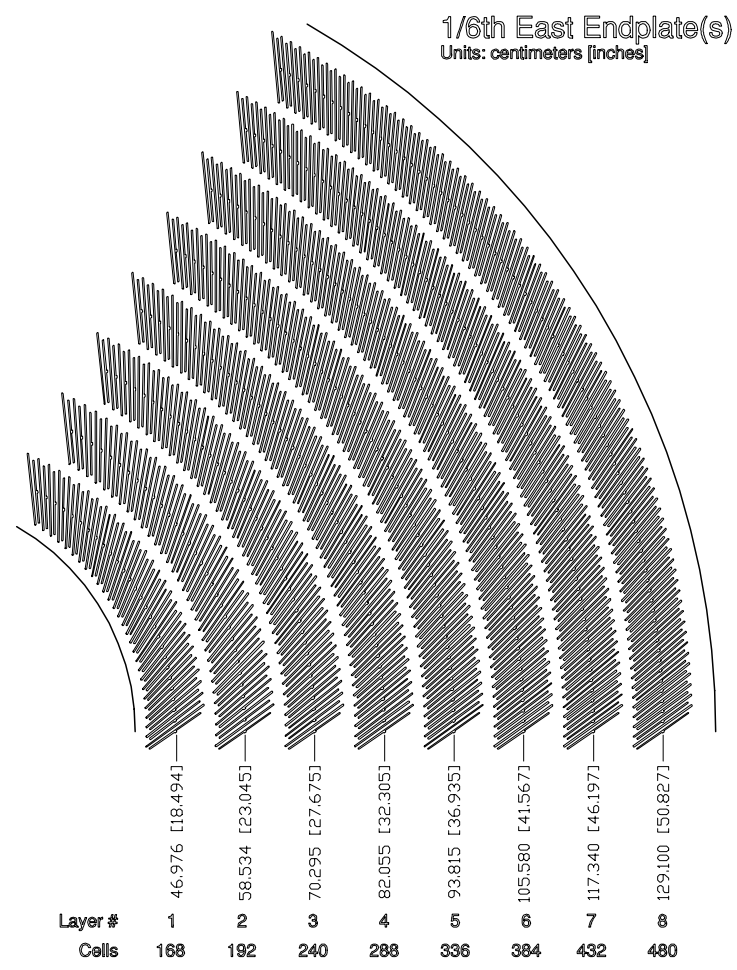

Figure 2.6: Superlayers of the COT, alternating between stereo and axial superlayers.

Primary electrons resulting from the ionisation of the gas move towards the sense wires. In the vicinity of the wires, the $\frac{1}{r}$ dependence of the electric field causes them to accelerate and liberate more electrons, subsequently resulting in an 'avalanche' near the anode which amplifies the signal and is registered by the sense wires. The position of hits on the sense wires allows the track of the charged particle to be reconstructed and its curvature measured. Since curvature is inversely proportional 
to the transverse momentum $\left(p_{\mathrm{T}}\right)$ of the track, this allows the transverse momentum of the particle to be determined.

The tracking resolution of the COT is given by

$$
\frac{\sigma\left(p_{\mathrm{T}}\right)}{p_{\mathrm{T}}} \sim 0.15 \% \times p_{\mathrm{T}}
$$

where $p_{\mathrm{T}}$ is measured in $\mathrm{GeV}$.

\subsubsection{Calorimeter System}

The CDF calorimeter system is subdivided into the electromagnetic calorimeter and the hadronic calorimeter. They are designed to absorb the energies of different types of particles and convert them to a measurable signal. Electrons produce bremsstrahlung in materials thus showering quickly and losing their energy early. Their shower is therefore mostly contained in the electromagnetic calorimeter. However, hadronic jets shower later and leave a significant energy deposit in the hadronic calorimeter. Muons, on the other hand, only deposit small amounts of energy in both the calorimeters. This is illustrated in Figure 2.7.

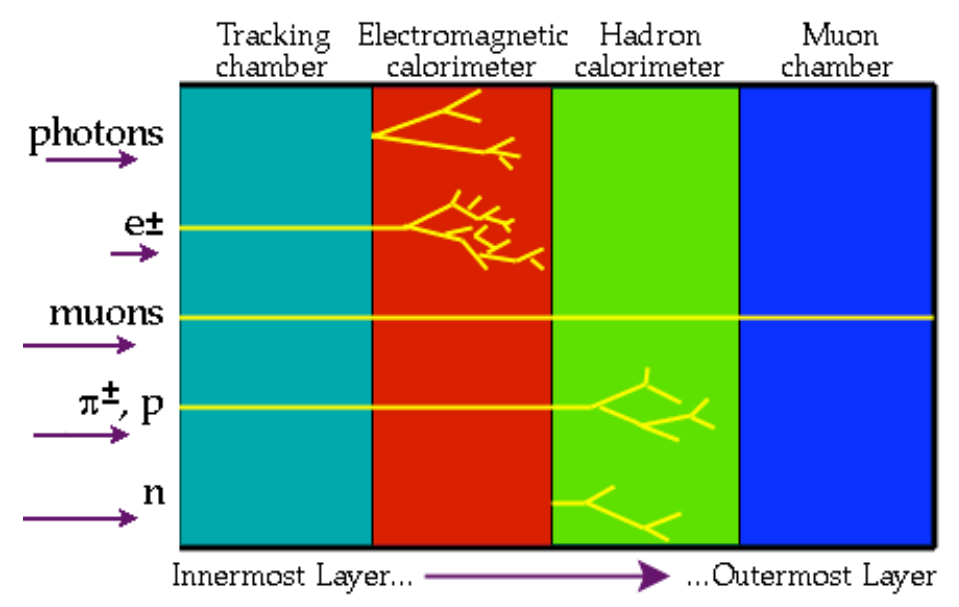

Figure 2.7: A schematic diagram showing where different particle types deposit their energy in the CDF calorimeter system. 


\section{Electromagnetic Calorimeter}

The electromagnetic (EM) calorimeter [16] is divided into two physical sections, the central electromagnetic calorimeter (CEM) which covers the region $|\eta|<1$ and the plug electromagnetic calorimeter (PEM), covering the region $1.1<|\eta|<3.64$.

The CEM is split at $\eta=0$ into 2 halves. Each half is segmented into 24 wedges with each wedge subtending $15^{\circ}$ in $\phi$ and containing 10 electromagnetic towers with projective geometry, such that the centre of the face of the tower points towards the nominal interaction point. A schematic diagram of a calorimeter wedge, with the towers labelled from 0 to 9 is shown in Figure 2.8. Each tower is made from layers of lead sheets interspersed with polystyrene scintillator.

An electron entering dense sheets of lead in the calorimeter produces a photon by bremsstrahlung, which subsequently converts into an electron-positron pair, thus initiating an electromagnetic cascade. Electrons generated in this cascade enter the scintillator layers and produce light which is collected by photomultiplier tubes. The number of particles produced and hence the amount of light collected is proportional to the energy of the incoming electron or photon. The calorimeter has a thickness of $32 \mathrm{~cm}$ which translates to 18 radiation lengths. This ensures that approximately 99.7\% of an electron's energy will be deposited in the calorimeter.

A proportional strip chamber (CES) is inserted between the 8th layer of lead and the 9th layer of scintillator. Its location is at a depth of 6 radiation lengths and corresponds to the depth at which typical showers are expected to reach their maximum transverse extent. The CES has anode wires in the $r-\phi$ plane and cathode strips in $z$. Charge is collected on these wires and strips and since the amount of charge deposited is proportional to the energy of the showering particle, this information is then used to construct a 3-D picture of the precise position and transverse 


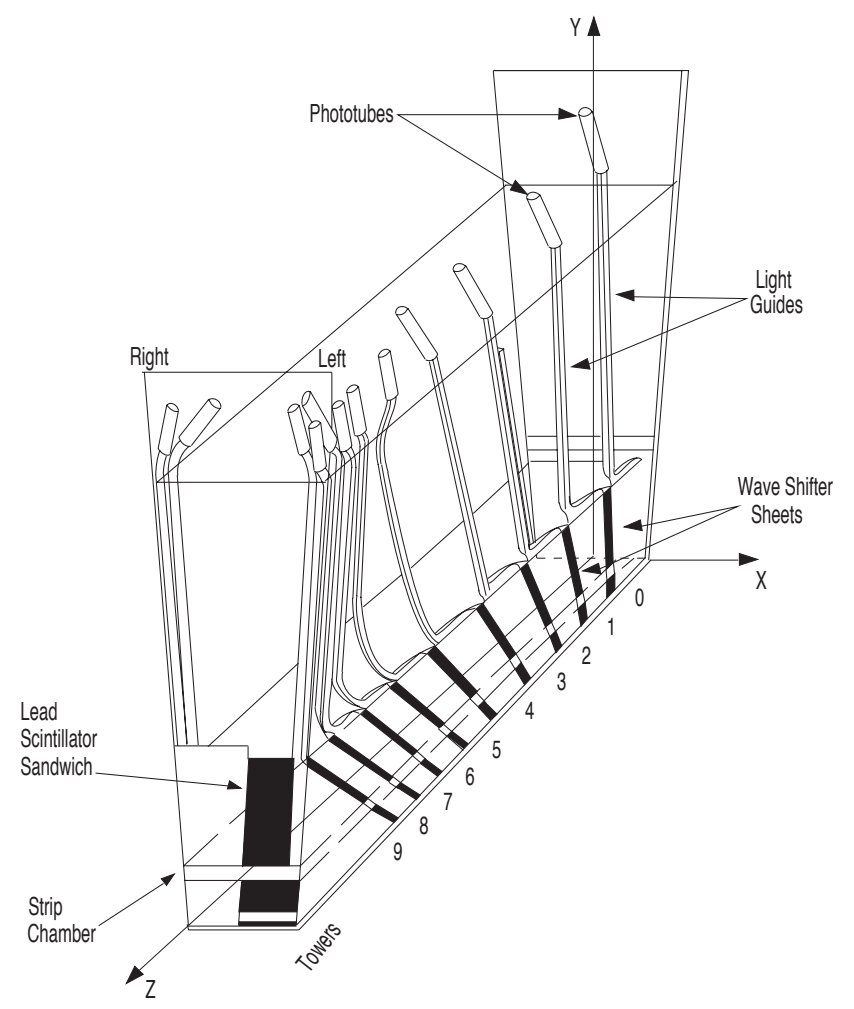

Figure 2.8: A schematic diagram of a central calorimeter wedge showing the ten electromagnetic calorimeter towers.

development of the shower in the CEM. This shower topology information is useful to distinguish between electrons or photons and light hadrons (e.g $\pi$ or $K$ ), since the transverse development of the showers is different for these particles. The position of the shower as measured in the CES is used for matching the EM cluster to a track in the COT. It has a position resolution of $2 \mathrm{~mm}$ at $50 \mathrm{GeV}$.

The plug electromagnetic calorimeter covers the high $\eta$ region $(1.1 \leq|\eta| \leq 3.6)$. It has a similar composition to the CEM, consisting of a stack of lead and scintillator sheets read out by phototubes. At lower values of $|\eta|$, the plug calorimeter is azimuthally segmented into 48 wedges, each subtending $7.5^{\circ}$ in $\phi$. At higher values of $|\eta|$, the segmentation resembles that of the CEM, with 24 wedges, each subtending $15^{\circ}$ in $\phi$. The PEM also has a shower maximum detector (PES) located at 


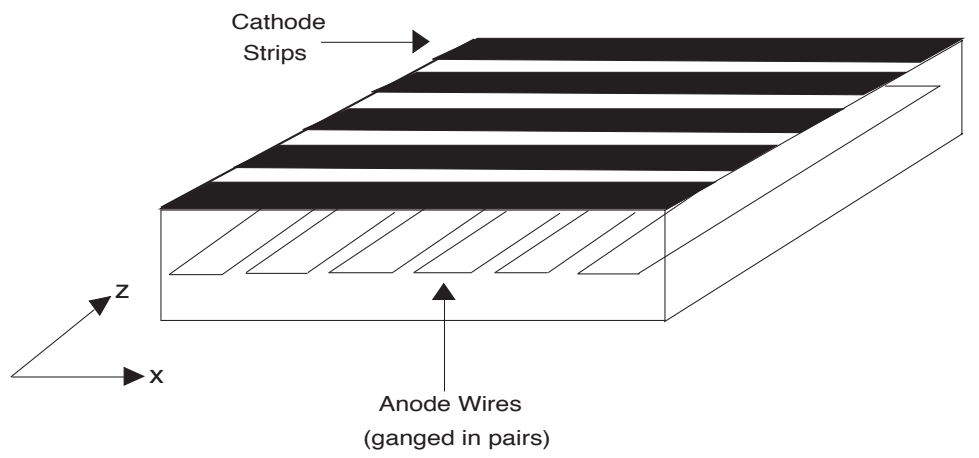

Figure 2.9: Schematic diagram of the proportional strip chamber (CES) in the central calorimeter.

approximately 6 radiation lengths.

\section{Hadronic Calorimeter}

The hadronic calorimeter [17] is situated just outside in radius to the EM calorimeter and is divided into 3 physical sections, the Central Hadronic Calorimeter (CHA), the Plug Hadronic Calorimeter (PHA) and the Wall Hadronic Calorimeter (WHA) which cover different regions of pseudorapidity. The CHA covers the region $|\eta|<0.9$ and the PHA covers the region $1.1<|\eta|<3.6$. The WHA is placed in the gap between the CHA and PHA, covering the region $0.7<|\eta|<1.3$.

The hadronic calorimeter is made of alternating layers of steel and scintillator. They are similarly segmented to the EM calorimeter with 24 wedges subtending $15^{\circ}$ in $\phi$, except for the PHA which follows the same segmentation as the PEM. The energy resolutions of these various components of the calorimeter are determined using incident electrons of energy $50 \mathrm{GeV}$ for the electromagnetic calorimeter and incident pions of energy $50 \mathrm{GeV}$ for the hadronic calorimeter in a test beam run. The resolutions obtained are given in Table 2.1. 


\begin{tabular}{|lr|}
\hline Detector system & Resolution \\
\hline \hline CEM & $\frac{\sigma(E)}{E} \approx 13.5 \% / \sqrt{E_{\mathrm{T}}}(\mathrm{GeV}) \oplus 2 \%$ \\
$\mathrm{PEM}$ & $\frac{\sigma(E)}{E} \approx 14.4 \% / \sqrt{E}(\mathrm{GeV}) \oplus 0.7 \%$ \\
$\mathrm{CHA}$ & $\frac{\sigma(E)}{E} \approx 50 \% / \sqrt{E}(\mathrm{GeV})$ \\
$\mathrm{CES}$ & $2 \mathrm{~mm}$ at $50 \mathrm{GeV}$ \\
\hline
\end{tabular}

Table 2.1: Resolutions of the calorimeter sub-systems (where $\oplus$ denotes addition in quadrature).

\subsubsection{Muon Chambers}

Muons, due to their larger mass compared to electrons, produce less bremsstrahlung and therefore their interaction with the material of the calorimeters does not produce a shower. They traverse the entire depth of the detector without leaving a significant energy deposit in either the electromagnetic or hadronic calorimeters. Muon chambers therefore form the outermost detectors.

The muon detector system consists of 4 chambers comprising of scintillating material and layers of drift tubes containing a gas mixture of ethane and argon. Muons entering the detector ionise the gas in the chambers leaving a trail of ions and electrons along their trajectory and inducing light pulses in the scintillator panels which are collected by the PhotoMulitplier Tubes (PMTs). Information from the drift tubes and the scintillators is combined to calculate the trajectory of the muon.

The central muon system (CMU) [19] has a cylindrical shape and is located behind the CHA covering the pseudorapidity region $|\eta|<0.6$. It comprises of a four layer drift chamber where the layers are divided into rectangular cells. Each cell has a single sense wire which is attached to a TDC to get timing information with a single sense wire in each cell.

The central muon upgrade (CMP) detector also covers the $|\eta|<0.6$ region and complements the CMU. It is preceded by $60 \mathrm{~cm}$ of steel to absorb fake muons and reduce background. Muons that give hits in the CMU are also generally required to have a cor- 
responding hit in the CMP and are known as CMUP muons. The matching hits in the CMU and CMP are known as muon 'stubs'.

The central muon extension (CMX) detector extends the coverage of the muon system. It covers the region $0.6 \leq|\eta| \leq 1$ with a slight overlap with the CMU in the region $|\eta|=0.6$. It consists of arches arranged at each end of the central detector. Scintillator plates (CSX) are mounted on the inside and outside of the CMX detector and the excellent timing resolution of the CSX allows the rejection of backgrounds from interactions in the beam pipe that are not in coincidence with proton-antiproton collisions.

The Intermediate Muon system (IMU) covers the region $1.0 \leq|\eta| \leq 1.5$. The $\eta-\phi$ coverage of the muon system is shown in Figure 2.10.

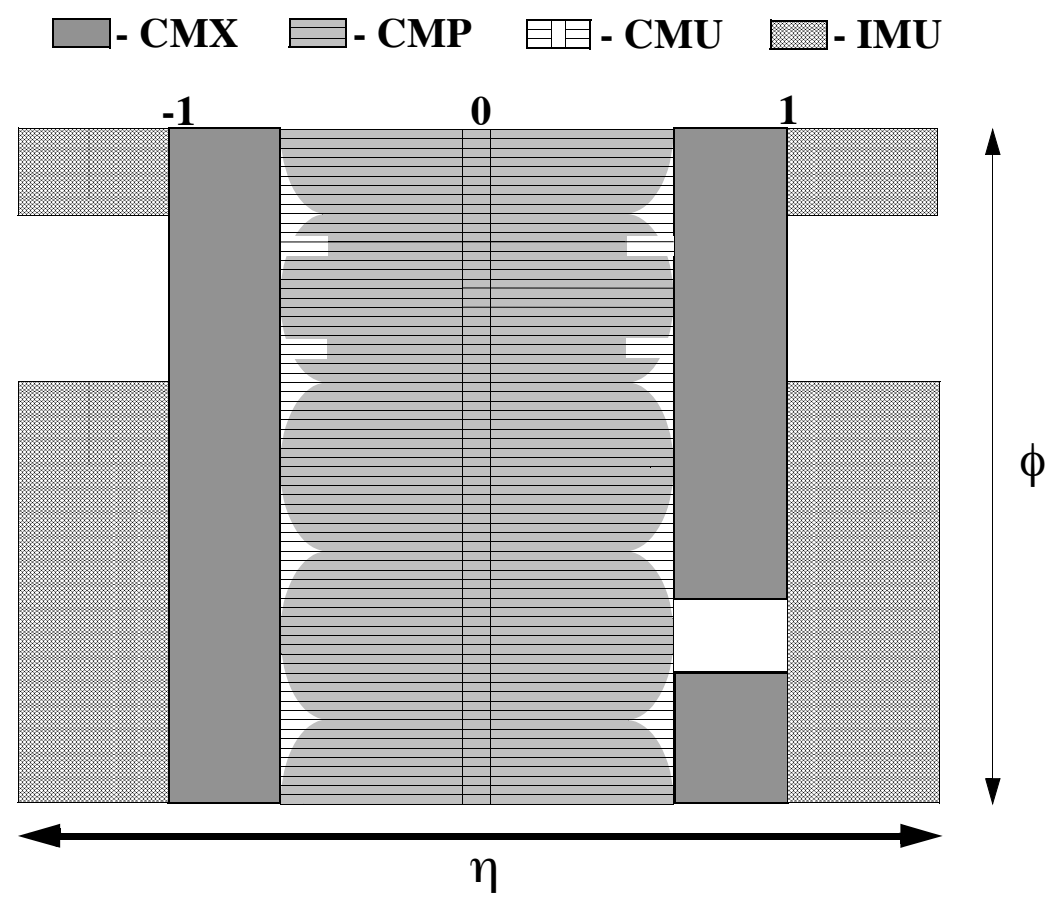

Figure 2.10: Schematic diagram of the sub-detectors comprising the muon chamber and their coverage in $\eta$ and $\phi$. 


\subsection{The Trigger System}

The proton-antiproton collisions at CDF occur every $396 \mathrm{~ns}$ giving a collision rate of 2.5 $\mathrm{MHz}$ which is far too large to enable all the events to be written to tape. The collisions produce a range of physics processes, only a fraction of which contain physics that is considered interesting against a large background of hadronic activity. In order to store as many interesting physics events as possible to tape, the event information is processed through a three level trigger system that reduces the amount of data recorded and stored to an acceptable amount. The three level trigger evaluates the information provided by the various sub-detectors and decides whether an event is interesting enough to be written to tape, with each level utilising more sophisticated algorithms and taking longer to make a decision on an event.

Information from the front end electronics of the various sub detectors first goes to the Level 1 trigger. The Level 1 trigger is a hardware trigger and makes use of simple and fast algorithms that take readouts from the calorimeter, tracking chamber and muon detectors to make a decision within $5.5 \mu \mathrm{s}$ on whether an event is interesting enough to be passed to Level 2. The Level 1 trigger reduces the data rate from $2.5 \mathrm{MHz}$ to approximately 30 $\mathrm{kHz}$ or less.

The Level 2 trigger is a software trigger that receives information at a rate of $30 \mathrm{kHz}$ or less and uses more sophisticated algorithms than Level 1 to perform the clustering of calorimeter towers into calorimeter objects which can later be identified as electrons, photons or jets. Information from the CES detector is also used to identify electrons and photons and reject background. Secondary vertices are found using the silicon vertex detector and track impact parameters are computed using the Level 1 tracking information. The Level 2 trigger has a decision time of about $30 \mu$ s per event and reduces the data rate by a factor of 100 . 
The Level 3 trigger system is a computer farm of Linux PCs that use the full readout of the detector to reconstruct events using a simplified version of the CDF offline reconstruction software. The Level 3 trigger reduces the rate from $300 \mathrm{~Hz}$ to about $75 \mathrm{~Hz}$ which is then written to storage tape.

The $\mathrm{W} \rightarrow l \nu$ and $\mathrm{Z} \rightarrow l^{+} l^{-}$processes are triggered using the high $p_{\mathrm{T}}$ electrons and muons in the decay of the boson. The detailed requirements at each level of the electron and muon trigger is described in the following.

\subsubsection{Electron Trigger}

The trigger path used for high $p_{\mathrm{T}}$ electrons is the ELECTRON_CENTRAL_18 trigger. The requirements at each level of the three-tier trigger are:

- Level 1 : requires the electromagnetic transverse energy of a calorimeter cluster to exceed $8 \mathrm{GeV}$ and the ratio of the energy in the hadronic and electromagnetic calorimeter $\left(E_{\text {had }} / E_{\text {em }}\right)$ to be less than 0.125 . A track reconstructed using the extremely fast tracker (XFT) [20] is required to have a $p_{\mathrm{T}}$ greater than $8 \mathrm{GeV}$ and to point to the calorimeter cluster. The XFT uses hit information from the axial superlayers of the COT to construct short segments of tracks which are then combined to form a full reconstructed track.

- Level 2 : requires an electromagnetic cluster with transverse energy greater than 16 $\mathrm{GeV}$ and a $E_{\text {had }} / E_{\text {em }}$ ratio less than 0.125 . It also requires an XFT track with $p_{\mathrm{T}}$ greater than $8 \mathrm{GeV}$ to point to the Level 2 calorimeter cluster.

- Level 3 : the final stage of the trigger path requires an electromagnetic cluster with transverse energy greater than $18 \mathrm{GeV}$ and a $E_{\text {had }} / E_{\text {em }}$ ratio less than 0.125 . It also requires a fully reconstructed $\mathrm{COT}$ track to extrapolate to the calorimeter cluster and have $p_{\mathrm{T}}$ greater than $9 \mathrm{GeV}$. 


\subsubsection{Muon Trigger}

Two trigger paths are used to select high $p_{\mathrm{T}}$ muons in the $\mathrm{W}$ mass and width analyses, MUON_CMUP18 if the muon has hits in both the CMU and CMP muon chambers and MUON_CMX18 if it has hits in the CMX. The following criteria are applied at each level of the trigger path:

- Level 1 : the MUON_CMUP18 trigger requires hits in the CMU chamber that are spatially matched to an XFT track with $p_{\mathrm{T}}$ greater then $4 \mathrm{GeV}$. The muon is also required to produce hits in the $\mathrm{CMP}$ chamber with the direction of the hits consistent with those in the CMU.

The MUON_CMX18 trigger requires an XFT track with $p_{\mathrm{T}}$ greater than $8 \mathrm{GeV}$ pointing to a stub in the CMX chamber and hits in the CSX scintillator counters.

- Level 2 : the track $p_{\mathrm{T}}$ requirement is raised to $8 \mathrm{GeV}$ for the MUON_CMUP18 trigger and to $10 \mathrm{GeV}$ for the MUON_CMX18 trigger.

- Level 3 : the MUON_CMUP18 trigger requires a track pointing to stubs in both the CMU and CMP chambers with $p_{\mathrm{T}}$ greater than $18 \mathrm{GeV}$. The distance between the extrapolated track and the position of the stubs in the CMU and CMP chambers must be less than $10 \mathrm{~cm}$ and $20 \mathrm{~cm}$ respectively.

The MUON_CMX18 trigger requires a track with $p_{\mathrm{T}}$ greater than $18 \mathrm{GeV}$ matched to a stub in the CMX detector. The distance between the extrapolated track and the stub position is required to be less than $10 \mathrm{~cm}$. 


\section{Chapter 3}

\section{The Mass and Width of the W Boson}

\subsection{Motivation}

The mass and width of the $\mathrm{W}$ boson are fundamental electroweak parameters within the Standard Model. Whereas the mass and width of the $\mathrm{Z}$ boson have been measured to very high precision by the LEP experiments which together collected 17 million $\mathrm{Z}$ boson events, the properties of $\mathrm{W}$ bosons are less well measured. However, with the Tevatron currently operating as a W boson factory and producing copious amounts of these particles, it is an ideal place to study their properties and measure their parameters to an unprecedented level of precision.

\subsubsection{W Mass}

The mass of the $\mathrm{W}$ boson can be derived from its relationship with other Standard Model quantities which have been precisely determined; namely the Fermi constant $G_{F}$, the mass of the $\mathrm{Z}$ boson $\left(M_{Z}\right)$ and the electromagnetic coupling constant $\alpha$ at the renormalisation energy scale $Q=M_{Z}$. The $\mathrm{W}$ mass, at leading order, is related to the weak coupling constant, $g$ via the following relation

$$
g^{2}=(8 / \sqrt{2}) G_{F} M_{W}^{2}
$$

where $g$ can be written in terms of the electromagnetic coupling $e$ and the weak mixing angle $\theta_{W}$

$$
g \sin \theta_{W}=e .
$$


Substituting 3.1 into 3.2 and using the relation, $\alpha=e^{2} / 4 \pi$, gives the expression for the W boson mass as

$$
M_{W}=\left(\frac{\pi \alpha}{\sqrt{2} G_{F}}\right)^{1 / 2} \frac{1}{\sin \theta_{W} \sqrt{1-\Delta r}}
$$

where $\Delta r$ includes all the radiative corrections to the $\mathrm{W}$ propagator.

A precise determination of the $\mathrm{W}$ boson mass requires the inclusion of higher order diagrams which involve loop corrections to the tree level calculation, where the loops can be populated by any particle in the theory. There are two contributions to $\Delta r$ that are particularly significant. The correction from loops involving fermions depend on the squared fermion mass difference and it is therefore dominated by the loop containing the heaviest fermions, the top and bottom quarks, shown in Figure 3.1(a). The correction to the $\mathrm{W}$ mass from the $t \bar{b}$ loop is given by $\Delta M_{W} \propto M_{t}^{2}-M_{b}^{2}$ [21]. Another important contribution to $\Delta r$ arises from loops containing the Higgs boson, where the W radiates and then reabsorbs a virtual Higgs boson as shown in Figure 3.1(b). This contribution depends on the logarithm of the mass of the Higgs boson, $\Delta M_{W} \propto \ln \left(M_{H}\right)$.

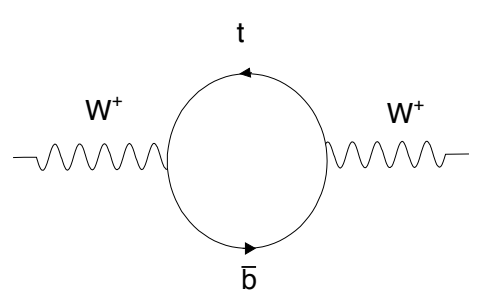

(a)

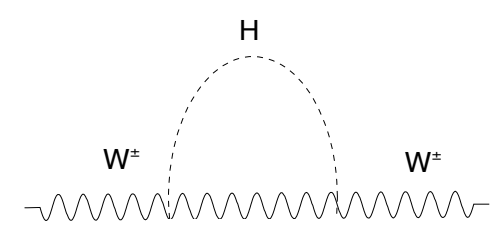

(b)

Figure 3.1: Radiative corrections to the $\mathrm{W}$ boson propagator from the (a) $t \bar{b}$ loop and (b) the Higgs boson loop.

All the quantities excluding $\Delta r$ in Equation 3.3 have been measured to a very high level of precision. A precise measurement of the $\mathrm{W}$ mass probes these radiative corrections and 
in conjunction with the top mass, can indirectly constrain the mass of the Higgs boson. The first measurement of the W mass in Run II of the Tevatron at CDF used $200 \mathrm{pb}^{-1}$ of data and produced the single most precise published measurement of this quantity to date with $M_{W}=80413 \pm 48 \mathrm{MeV}$ [22]. When this measurement is included in the world average, the $\mathrm{W}$ mass becomes $M_{W}=80.399 \pm 0.025$ [24]. This uncertainty is further reduced to $23 \mathrm{MeV}$ when the new DØ $\mathrm{W}$ mass measurement [25] is included. The results of precision measurements of the $\mathrm{W}$ mass from various experiments at LEP2 and the Tevatron are shown in Figure 3.2.

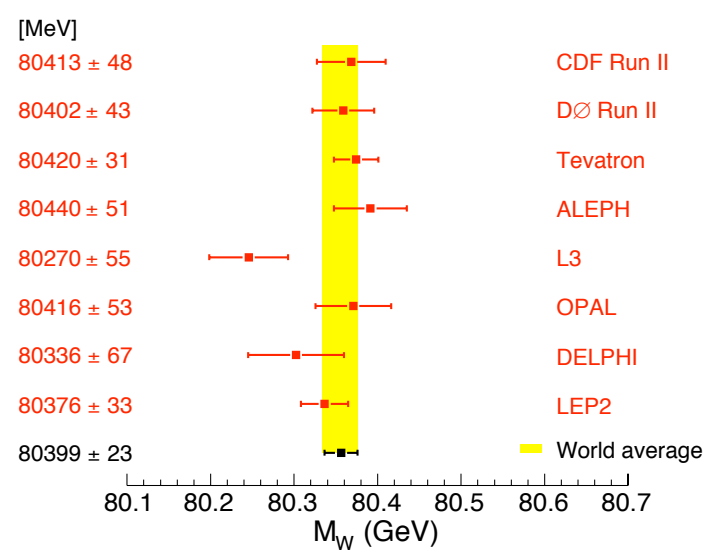

Figure 3.2: The mass of the $\mathrm{W}$ boson as measured by the ALEPH [30], L3 [31], OPAL [32] and DELPHI [33] experiments at LEP2 and the CDF and DØ [35] experiments at the Tevatron, including the current world average value.

The latest measurements of the top mass from the Tevatron have been combined to give a precision of $1.2 \mathrm{GeV}$ [27] on this quantity. This leads to a smaller uncertainty on the Higgs mass as compared to that from the current world average measurement of the $\mathrm{W}$ mass mentioned above. The $\mathrm{W}$ mass is therefore the dominant error on the estimation of the Higgs boson mass and needs to be known to a precision of less than $7 \mathrm{MeV}$ to have an equivalent effect on the Higgs mass uncertainty as that provided by the top mass. The relationship between the masses of the $\mathrm{W}$ boson, the top quark and the Higgs boson is shown in Figure 3.3. The green shaded region represents the Higgs 
mass range allowed by the Standard Model where the lower limit has been set from direct searches at LEP which have excluded a Higgs boson of mass below $114 \mathrm{GeV}[28]$ and the upper limit is set by theoretical constraints such as the validity of the Standard Model before pertubation theory breaks down. The red contour shows the predictions from older electroweak measurements from LEP1 and SLD (Stanford Linear collider Detector) [29] while the blue ellipse shows the most likely values of the top mass and $\mathrm{W}$ mass at the $68 \%$ confidence level using LEP2 [34] and Tevatron data including the latest top and W mass measurements. This ellipse has become considerably smaller with the increase in precision on these measurements and it is evident that a reduction in the $\mathrm{W}$ mass uncertainty will have the largest effect in further shrinking the ellipse and constraining the Higgs mass. Figure 3.4 shows the $\Delta \chi^{2}$ obtained from a fit to all measured electroweak observables as a function of the Standard Model Higgs mass. The blue band represents the effect of theoretical uncertainties on the fitted observables. The plot shows that the most probable value for the Higgs mass is $90 \mathrm{GeV}$ in the Standard Model with an asymmetric error, $M_{H}=90_{-27}^{+36} \mathrm{GeV}$. Including the new DØ Run II measurement of the W mass [25], given in Figure 3.2, in the electroweak fits gives a Higgs mass value of $M_{H}=87_{-26}^{+35}[36]$.

\subsubsection{W Width}

The width of the $\mathrm{W}$ boson can be precisely predicted in the Standard Model in terms of its mass and coupling. The partial width, $\Gamma(W \rightarrow e \nu)$, at leading order in the Standard Model is predicted to be [44]

$$
\Gamma_{e \nu}=\frac{G_{F} M_{W}^{3}}{6 \pi \sqrt{2}}\left(1+\delta_{W}\right)
$$

where $\delta_{W}$ denotes radiative corrections to the Born level expression. This number is small (less than $0.5 \%[44]$ ) since most of the corrections have already been absorbed into $G_{F}$ and $M_{W}$. Using the value of $G_{F}$ measured in muon decay [23], $G_{F}=1.16637 \times 10^{-5} \mathrm{GeV}^{-2}$ 


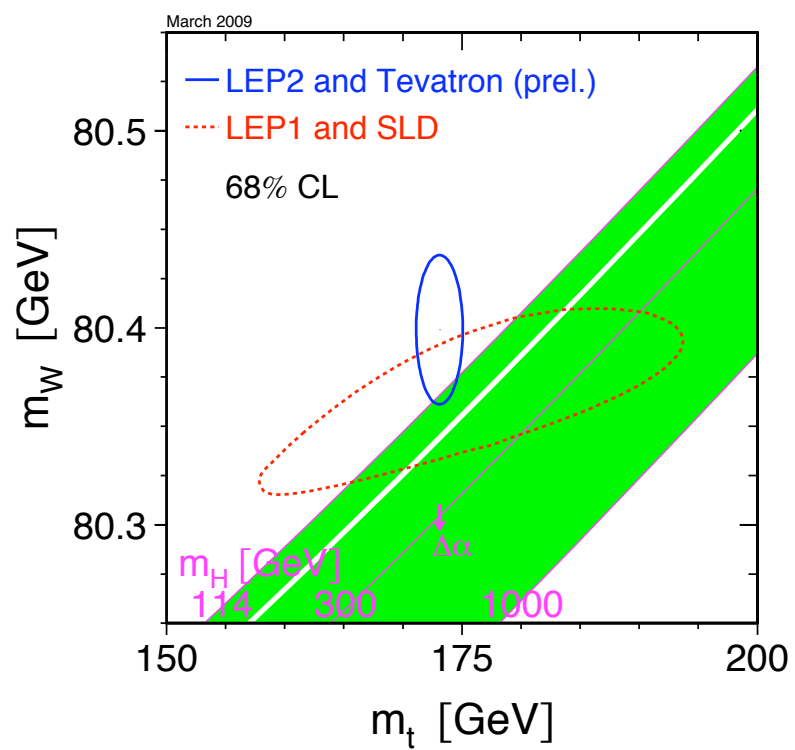

Figure 3.3: Constraints on the Higgs boson mass as a function of the $\mathrm{W}$ boson and top quark masses as measured in data (shown by ellipses) and predicted by theory.

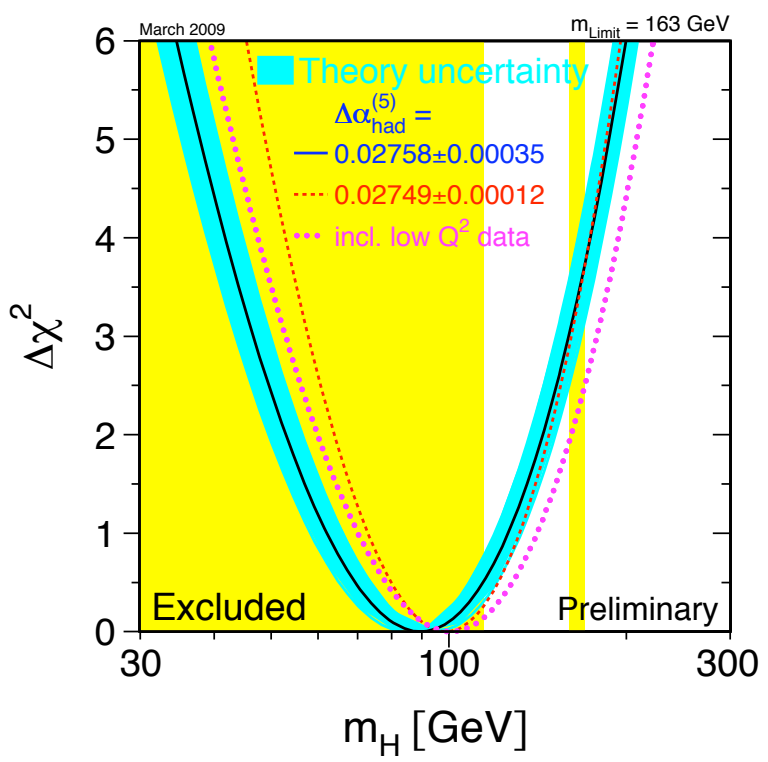

Figure 3.4: The $\Delta \chi^{2}$ from all electroweak precision measurements as a function of the Higgs mass. 
and the present world average value of $M_{W}, M_{W}=80.399 \pm 0.025 \mathrm{GeV}$, the partial decay width is predicted to be

$$
\Gamma_{e \nu}=226.47 \pm 0.25 \mathrm{MeV}
$$

This partial width can be divided by the branching ratio [44]

$$
B(W \rightarrow l \nu)=1 /\left(3+6\left(1+\alpha_{s}\left(M_{W}\right) / \pi+O\left(\alpha_{s}^{2}\right)\right)\right.
$$

to obtain the total width of the $\mathrm{W}$ boson

$$
\Gamma_{W}=2.093 \pm 0.002 \mathrm{GeV}
$$

which shows that the $\mathrm{W}$ width is predicted to a precision of less than $0.1 \%$ within the Standard Model. A precision measurement of this quantity therefore provides an incisive test of the Standard Model. In addition, the similarities between the mass and width measurements make it a very useful cross-check of that measurement. The $\mathrm{W}$ width has been measured at various experiments and the results until 2008 are shown in Figure 3.5.

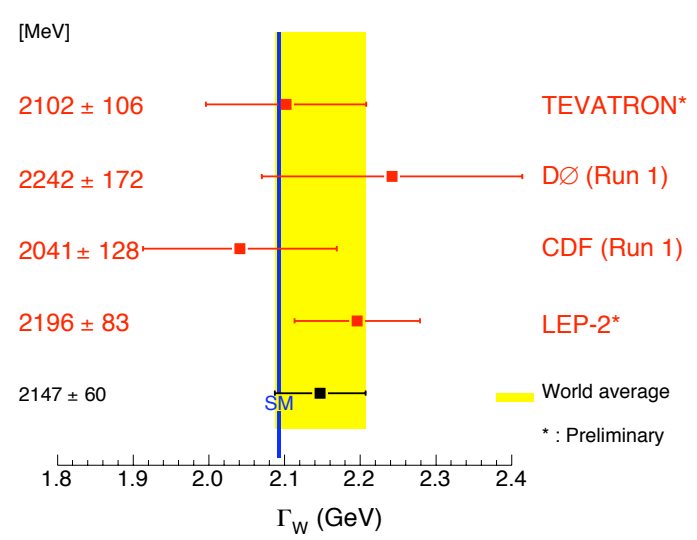

Figure 3.5: The width of the $\mathrm{W}$ boson as measured by the LEP and Tevatron experiments. The Tevatron and world average values represent the results before the measurement described in this thesis was incorporated.

\subsection{Measurement Strategy}

The production of $\mathrm{W}$ bosons at the Tevatron proceeds predominantly $(\sim 80 \%)$ via the annihilation of a valence quark from the proton and a valence antiquark from the antipro- 
ton. The quark and antiquark carry a fraction $x_{1}$ and $x_{2}$ of the proton and antiproton's momentum respectively. The centre-of-mass energy available to produce the $\mathrm{W}$ boson is related to the total centre-of-mass energy of the $p \bar{p}$ system in the following way, $\hat{s}=x_{1} x_{2} s$, where $\hat{s}$ is the invariant mass of the two interacting partons and $s$ is the square of the centre-of-mass energy of the $p \bar{p}$ system. The probability of finding a certain parton within a proton (antiproton) carrying momentum fraction $x$ of the proton (antiproton) momentum is governed by parton distribution functions (PDFs). $\mathrm{A}^{+}$can be produced by the quark and antiquark combination of $u \bar{d}$. The $u$ valence quarks on average carry more momentum than $d$ valence quarks such that the collision is momentum asymmetric and results in the $\mathrm{W}$ boson acquiring a net longitudinal momentum.

W bosons can decay via both leptonic and hadronic decay channels. The hadronic channels account for two-thirds of the $\mathrm{W}$ decay branching ratio owing to the three-fold colour degeneracy of the quarks and each leptonic channel accounts for one-ninth of the branching ratio. At hadron colliders a large background from QCD processes resulting in two or more jets in the final state swamps the signal process in the hadronic channel. For the $\mathrm{W}$ mass and width measurements presented, only the electron and muon decay modes have been considered. These provide relatively clean experimental signatures for detection. The $\mathrm{W}$ decay to $\tau \nu$ is harder to identify as the $\tau$ can decay both leptonically and hadronically. ${ }^{1}$

At lowest order (shown in Figure 3.6(a)), the V-A nature of the weak interaction results in the angular distribution of the decay leptons being described by

$$
\frac{d \sigma}{d \cos \theta} \propto(1-\lambda q \cos \theta)
$$

where $q$ represents the charge of the lepton, $\lambda$ is the helicity of the $\mathrm{W}$ boson and $\theta$ is the angle between the charged lepton and the proton beam direction in the $\mathrm{W}$ rest frame.

\footnotetext{
${ }^{1}$ Unless otherwise stated, all subsequent discussions of particles also implies reference to their antiparticles.
} 


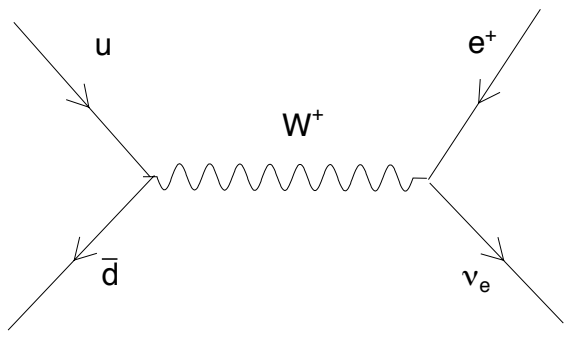

(a)

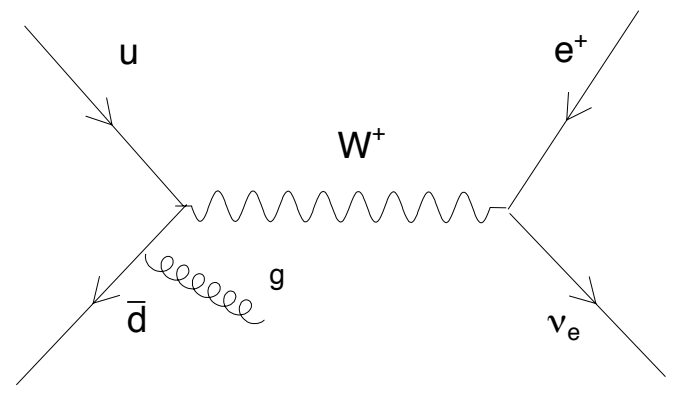

(b)

Figure 3.6: Feynman diagram for (a) lowest order production and decay of a $\mathrm{W}^{+}$boson and (b) next to leading order $\mathrm{W}^{+}$boson production.

At lowest order, the spin of the $\mathrm{W}$ boson is completely aligned with the direction of the incoming antiquark and quark so $\lambda= \pm 1$ as shown in Figure 3.7. However, when higher
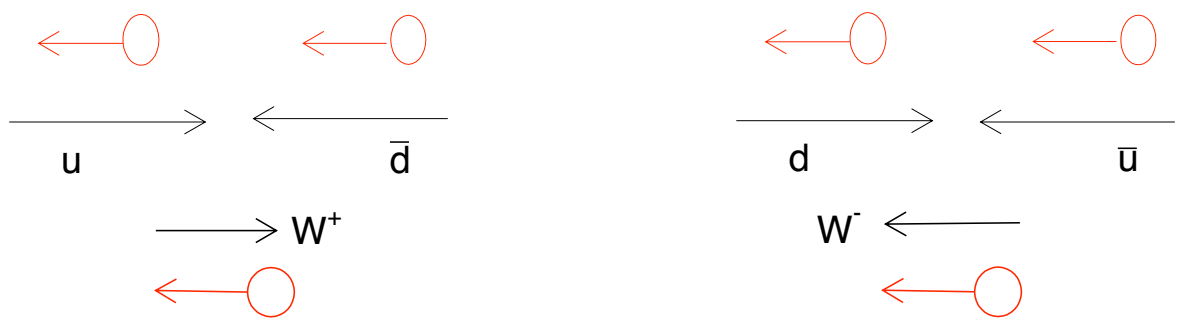

Figure 3.7: The direction of momenta and spin for the colliding partons and the $W^{ \pm}$boson are shown. The arrows with circles represent the direction of the particle spin.

order diagrams such as that shown in Figure 3.6(b) are included, where a quark or gluon is radiated by the initial state partons, the $\mathrm{W}$ boson acquires a transverse momentum $\left(p_{T}^{W}\right)$ to balance the transverse momentum of the initial state QCD radiation. This results in the spin of the $\mathrm{W}$ not being completely aligned in the direction of the antiproton (or proton) but rotated with respect to the beam axis. In addition, the angular momentum of the initial state is modified due to the emission of a spin 1 gluon. The lowest order angular decay distribution described by Equation 3.8 can be modified to account for these 
higher order effects and is given by [41]

$$
\frac{d \sigma}{d \cos \theta_{C S}} \propto\left(1-\lambda q \alpha_{1} \cos \theta_{C S}+\alpha_{2} \cos ^{2} \theta_{C S}\right)
$$

where the coefficients $\alpha_{1,2}$ are dependent on $p_{T}^{W}$ and $\theta_{C S}$ is the decay angle in the CollinsSoper frame [40]. The Collins-Soper frame is a rest frame of the $\mathrm{W}$ boson in which the $z$-axis bisects the angle between the proton momentum and the opposite of the antiproton momentum. A diagram of this is shown in Figure 3.8.

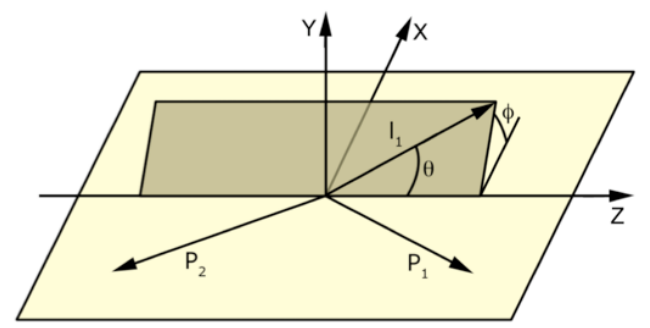

Figure 3.8: The Collins-Soper frame defining a $\mathrm{W}$ rest frame in which the $z$-axis is the bisector of the angle between the proton $\left(\mathrm{P}_{1}\right)$ momentum vector and the opposite of the antiproton $\left(\mathrm{P}_{2}\right)$ momentum vector. The charged lepton from the decay of the $\mathrm{W}$ boson has a polar angle $\theta$ and an azimuthal angle $\phi$.

In general, the mass of a particle produced in a resonance is measured using the invariant mass of its decay products. This is the case for the $\mathrm{Z}$ boson in $\mathrm{Z} \rightarrow l^{+} l^{-}$decays where the reconstructed charged leptons are used to construct the invariant mass of the $Z$ boson. For $\mathrm{W}$ bosons in $\mathrm{W} \rightarrow l \nu$ decays however, the neutrino is not reconstructed in the detector and any information on its energy is obtained by requiring momentum conservation and calculating the imbalance of energy in the final state. Remnants from the inelastic collision of the proton and antiproton travel close to the beam pipe carrying a substantial longitudinal momentum which due to the incomplete coverage of the detectors cannot be accurately measured. This makes it difficult to infer the longitudinal momentum of the neutrino and hence reconstruct an invariant mass for the $\mathrm{W}$ boson. Instead, a variable known as the transverse mass, $M_{T}$, is used which is analogous to the invariant mass except only the transverse components of the lepton momenta are used. It is defined 
as

$$
M_{T}=\sqrt{2 p_{T}^{l} p_{T}^{\nu}(1-\cos (\Delta \phi))}
$$

where $p_{T}^{l}$ is the transverse momentum of the charged lepton, $p_{T}^{\nu}$ is the transverse momentum of the neutrino and $\Delta \phi$ represents the angle between the charged lepton and the neutrino in the transverse plane.

Whereas $p_{T}^{l}$ can be directly reconstructed, $p_{T}^{\nu}$ is inferred by calculating the transverse momentum imbalance. This is done by measuring the transverse energy of all other energy deposits in the calorimeters which includes contributions from the initial state QCD radiation, remnants of the $p \bar{p}$ collision as well as energy from additional inelastic interactions within the same bunch crossing. Transverse energy from these additional sources is collectively referred to as the recoil.

The transverse mass spectrum is characterised by a smooth distribution with a sharply falling edge, also known as a Jacobian edge, which occurs at $M_{T}=M_{W}$ and the location of this edge is used to extract the mass of the W. A study of the distribution of W events as a function of the charged lepton transverse momentum $\left(p_{T}\right)$ can show how this edge arises. In the rest frame of the $\mathrm{W}$ boson, neglecting the masses of the decay products, the leptons are emitted back to back and carry half of the W mass, so $p=M_{W} / 2$. The transverse momentum of the lepton is given by

$$
p_{T}=(1 / 2) M_{W} \sin \theta \text {. }
$$

The differential cross-section in $p_{T}$ can then be written as

$$
\begin{aligned}
\frac{d \sigma}{d p_{T}} & =\frac{d \sigma}{d \cos \theta} \frac{d \cos \theta}{d p_{T}} \\
& =\frac{d \sigma}{d \cos \theta}\left|\frac{d \cos \theta}{d \sin \theta}\right| \frac{2}{M_{W}}
\end{aligned}
$$

where the term $\left|\frac{d \cos \theta}{d \sin \theta}\right|$ is called the Jacobian factor. Substituting Equation 3.11 into the above gives

$$
\frac{d \sigma}{d p_{T}}=\frac{d \sigma}{d \cos \theta}\left(\frac{2 p_{T}}{M_{W}}\right) \frac{1}{\sqrt{\left(M_{W}^{2} / 4\right)-p_{T}^{2}}}
$$


Equation 3.14 shows that the $p_{T}$ distribution has a singularity at $p_{T}=M_{W} / 2$. This divergence does not lead to an infinite cross-section because $M_{W}$ is distributed according to a Breit-Wigner shape (as described below), however, it does give rise to a strong peak with a sharply falling edge that is referred to as the Jacobian edge.

In principle, all three distributions, the $M_{T}, p_{T}^{l}$ and $p_{T}^{\nu}$ exhibit the Jacobian edge and can be used to extract the $\mathrm{W}$ mass. In the $M_{T}$ distribution this edge roughly coincides with the mass of the $\mathrm{W}$ and in the lepton transverse momentum distributions it occurs at $M_{W} / 2$. However, all three distributions are sensitive to different systematic effects.

The $M_{T}$ distribution has the main advantage of being relatively insensitive to the theoretical description of the $\mathrm{W}$ transverse momentum [37] but since it is dependent on $p_{T}^{\nu}$ and hence the recoil, it is sensitive to the modelling of the recoil response resulting in a significant smearing of the Jacobian edge due to the detector resolution and response. The effect is shown in Figure 3.9. The edge is also smeared due to the width of the W boson and it will be explained later how the region above the $\mathrm{W}$ pole is used to extract the $\mathrm{W}$ boson width.

The shape of the $p_{T}^{l}$ distribution is very sensitive to the transverse motion of the W boson causing a large smearing of the Jacobian edge. Since it is not directly dependent on $p_{T}^{\nu}$ it is relatively insensitive to the modelling of the recoil. These effects are illustrated in Figure 3.9.

The $p_{T}^{\nu}$ distribution is sensitive to both of the above described effects. It receives the same large correction due to the transverse momentum of the $\mathrm{W}$ boson as $p_{T}^{l}$ and is also significantly smeared due to the recoil. Extracting $M_{W}$ from this distribution therefore carries the largest systematic.

As such, the transverse mass spectrum is used to extract the $\mathrm{W}$ mass and the $p_{T}^{l}$ and $p_{T}^{\nu}$ distributions are generally used as cross-checks. 


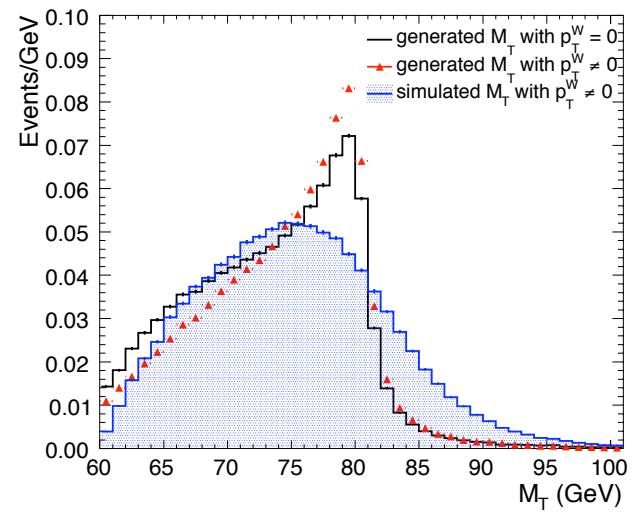

(a)

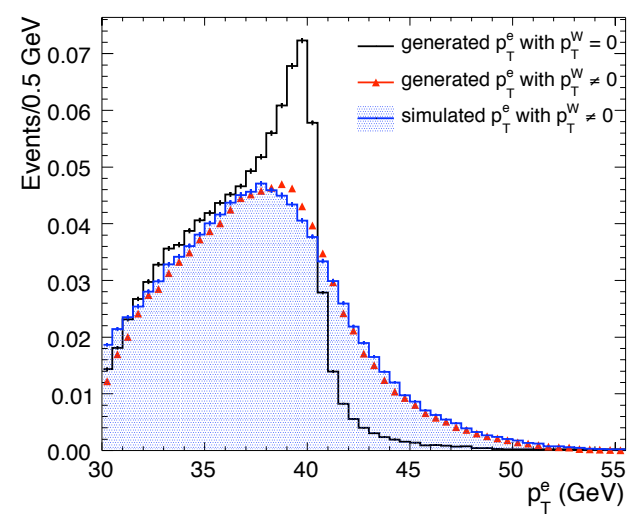

(b)

Figure 3.9: The (a) transverse mass spectrum and (b) electron transverse momentum distribution. The black line represents the shape of the distribution with $p_{T}^{W}=0$, the red points are with the correct $p_{T}^{W}$ distribution and the shaded region shows the shapes of the distributions including the effect of detector resolution and response.

As noted earlier, in the region above the pole mass the $M_{T}$ distribution is smeared by the finite width of the $\mathrm{W}$ boson. The $\mathrm{W}$ boson propagator factor $1 /\left(q^{2}-M_{W}^{2}\right)$, where $q$ is the 4-momentum of the boson, can be modified to include the effects of a finite $\mathrm{W}$ width by using $1 /\left(q^{2}-M_{W}^{2}+i q^{2} \Gamma / M_{W}\right)$. The relativistic Breit-Wigner parton level cross-section of the production of a $\mathrm{W}$ boson from $q \bar{q}$ and its subsequent decay to $l \nu$ can be written as

$$
\hat{\sigma}=12 \pi \frac{\hat{s}}{M_{W}^{2}} \Gamma_{q \bar{q}} \Gamma_{f} \frac{1}{\left(\hat{s}-M_{W}^{2}\right)^{2}+\left(\hat{s} \Gamma_{W} / M_{W}^{2}\right)^{2}}
$$

where $\Gamma_{q \bar{q}}$ and $\Gamma_{f}$ are the partial decay widths of the $\mathrm{W}$ boson into the initial and final states respectively and $q^{2}$ has been replaced by $\hat{s}$. In principle, it is possible to fit just above the peak region to extract the width, however, the $M_{T}$ distribution falls off quite rapidly close to the edge and is dominated by the detector resolution, in particular the recoil resolution. In the region away from the $\mathrm{W}$ pole, the shape of the Breit-Wigner falls off more slowly than the Gaussian shape of the detector resolution. This interplay between the Breit-Wigner and Gaussian shapes in the tail of the distribution allows the W width to be extracted and the effect is illustrated in Figure 3.10. The sensitivity of the tail of the transverse mass distribution to the $\mathrm{W}$ width is shown in Figure 3.11 where the 
simulation is used to produce $M_{T}$ distributions with different input widths. It is evident that in the region above $90 \mathrm{GeV}$ the shape of the distribution can distinguish between different $\mathrm{W}$ widths. Whereas the width is easier to measure at higher $M_{T}$ values, the decrease in events results in a higher statistical error. The fit region used to extract the W width, $M_{T}^{\text {low }}<M_{T}<200 \mathrm{GeV}$, is therefore varied with $M_{T}^{\text {low }}$ taking values $80,85,90,100$ and $110 \mathrm{GeV}$. The value of $M_{T}^{\text {low }}$ that minimises the combined statistical and systematical uncertainty on the width measurement is taken.

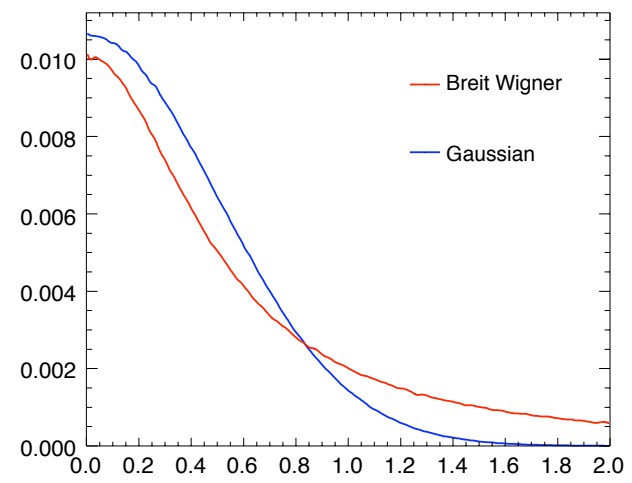

Figure 3.10: The comparison of the shape of the Breit-Wigner width component and the detector resolution described by a Gaussian. The slower fall off of the Breit-Wigner leads to the width component dominating the detector resolution in the tail of the distribution.

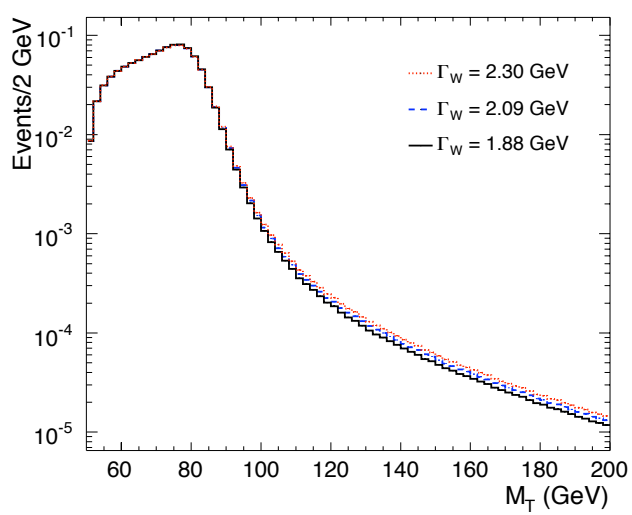

Figure 3.11: The $M_{T}$ distribution for different input values for the $\mathrm{W}$ width in the simulation. It shows that the tail of the distribution is most sensitive to the width.

The $\mathrm{W}$ width can also be extracted indirectly using the ratio of the $\mathrm{W} \rightarrow l \nu$ and $Z \rightarrow l l$ 
cross-sections

$$
\begin{aligned}
R & =\frac{\sigma(p \bar{p} \rightarrow W \rightarrow l \nu)}{\sigma(p \bar{p} \rightarrow Z \rightarrow l l)} \\
& \equiv \frac{\sigma(p \bar{p} \rightarrow W)}{\sigma(p \bar{p} \rightarrow Z)} \cdot \frac{B(W \rightarrow l \nu)}{B(Z \rightarrow l l)}
\end{aligned}
$$

where $\sigma(p \bar{p} \rightarrow W)$ and $\sigma(p \bar{p} \rightarrow Z)$ are the total production cross-sections and $B(W \rightarrow l \nu)$ and $B(Z \rightarrow l l)$ represent the branching ratios to the leptonic decay modes for the $\mathrm{W}$ and $\mathrm{Z}$ bosons respectively. The branching ratios can be written in terms of the partial and total decay widths such that

$$
R=\frac{\sigma(p \bar{p} \rightarrow W)}{\sigma(p \bar{p} \rightarrow Z)} \cdot \frac{\Gamma_{Z}}{\Gamma(Z \rightarrow l l)} \cdot \frac{\Gamma(W \rightarrow l \nu)}{\Gamma_{W}}
$$

The ratio of the $\mathrm{W}$ and $\mathrm{Z}$ total production cross-sections is taken from a theoretical calculation and the ratio $\Gamma_{Z} / \Gamma(Z \rightarrow l l)$ has been measured very precisely at LEP. Hence, using the Standard Model value for the partial width $\Gamma(W \rightarrow l \nu)$, a precise measurement of $R$ can be used to extract the total width $\Gamma_{W}$. An indirect measurement of the $\mathrm{W}$ width using this method yields $\Gamma_{W}=2.08 \pm 0.04 \mathrm{GeV}$ [39]. Whereas this is the most precise measurement of $\Gamma_{W}$, it assumes Standard Model values for the cross-section ratio and the partial width $\Gamma(W \rightarrow l \nu)$ and is thus not an independent validation of the Standard Model.

Both the $\mathrm{W}$ mass and $\mathrm{W}$ width measurements presented in this thesis utilise the $M_{T}$ distribution. Owing to incomplete detector coverage and complex acceptance and resolution effects, the shape of the $M_{T}$ distribution cannot be calculated. A dedicated detector simulation is used to model the $M_{T}$ distribution using parameters that have been tuned on $\mathrm{W}, \mathrm{Z}$ and minimum-bias data. An accurate modelling of $M_{T}$ requires a precise measurement of the response and resolution of the detector to charged leptons and the recoil, with the peak of the distribution being more sensitive to the response and the tail 
more sensitive to the resolution. The measurement technique and hence the simulation used to model the $M_{T}$ distribution for the mass and width are very similar. This thesis will focus in detail on the reconstruction and simulation of the recoil. This contributes as the largest systematic in the $\mathrm{W}$ width analysis and is an important part of the $\mathrm{W}$ mass measurement. The description of the recoil simulation for the $\mathrm{W}$ width analysis and its subsequent evolution to incorporate effects such as the increased instantaneous luminosity of the $\mathrm{W}$ mass dataset for the mass measurement will be discussed in Chapter 7. The recoil is modelled using the fully reconstructable decay of the $\mathrm{Z}$ boson to two charged leptons that are reconstructed in the central region of the calorimeter. In previous analyses, it has been assumed that modelling the recoil on $\mathrm{Z}$ events and subsequently using this model to simulate the recoil in $\mathrm{W}$ events where both leptons are not necessarily in the central region of the detector does not bias the recoil. This assumption has been tested for the first time for the $\mathrm{W}$ mass analysis by extending the recoil study to $\mathrm{Z} \rightarrow e^{+} e^{-}$ events where one electron is reconstructed in the central calorimeter and the other in the plug calorimeter and discussed in Chapter 8. The simulation of backgrounds in the event sample for the $\mathrm{W}$ mass measurement is described in Chapter 9. A summary of the event generation and simulation is given in Chapters 5 and 6 , albeit to avoid repetition, the specific details pertain to the $\mathrm{W}$ width measurement only. To conclude, the result of the W width measurement is presented in Chapter 10 followed by a discussion of the recoil systematics for the $\mathrm{W}$ mass. 


\section{Chapter 4}

\section{Event Selection}

The criteria used to select $\mathrm{W}$ and $\mathrm{Z}$ events are designed to reduce background contamination and define an event sample which is in a well understood part of the detector and can be accurately simulated. The electrons and muons from the decay are relatively easy to identify in the detector and they are selected using lepton identification and simple kinematic criteria. The $\mathrm{W}$ event is also required to satisfy some event selection requirements which constrain the missing energy and the recoil energy in the event.

The similarity in mass of the $\mathrm{Z}$ boson to the $\mathrm{W}$ boson and the complete reconstruction of its decay products in the detector make $\mathrm{Z} \rightarrow l^{+} l^{-}$events a very useful sample to calibrate the detector. $\mathrm{Z} \rightarrow l^{+} l^{-}$events are used extensively throughout the $\mathrm{W}$ mass and width analyses. As such, their event selection is designed to be as similar as possible to the $\mathrm{W} \rightarrow l \nu$ events to remove any bias due to the selection criteria.

In general, the kinematic cuts in the $\mathrm{W}$ mass analysis are raised to define a narrower kinematic region that ensures low background and an event sample that is well understood. In addition, the values for some of the kinematic and identification cuts are also chosen to match those used for the previous W mass measurement [22]. The more relaxed kinematic cuts in the $\mathrm{W}$ width analysis ensure that the measurement does not suffer from low statistics in the tail of the $M_{T}$ distribution whilst maintaining a high purity sample.

This chapter describes the criteria used to select $\mathrm{W}$ and $\mathrm{Z}$ events for the $\mathrm{W}$ mass and width analyses. 


\subsection{Electron Selection}

An electron leaves a track in the COT and produces an electromagnetic shower in the CEM with some leakage into the CHA. It is identified using readout information from all three sub-detectors.

The electron track in the COT is reconstructed using hits on the track. The track is required to originate at a $z$ position $\left(z_{0}\right)$ that is within $60 \mathrm{~cm}$ of the centre of the detector, accepting approximately $95 \%$ of events. The track is constrained to the beamspot and required to traverse at least three axial superlayers $\left(N_{\mathrm{seg}}^{\text {axial }}\right)$ and at least three stereo superlayers $\left(N_{\text {seg }}^{\text {stereo }}\right)$ with a minimum of seven hits in each layer. The transverse momentum of the beam constrained track $\left(p_{T}^{\mathrm{BC}}\right)$ reconstructed with COT hits only is required to be greater than $10 \mathrm{GeV}$ for the $\mathrm{W}$ width analysis and greater than $18 \mathrm{GeV}$ for the $\mathrm{W}$ mass analysis. The electromagnetic shower is required to be contained in a well instrumented and accurately modelled region of the calorimeter. The electron must be in towers $0-8$ of the calorimeter since electromagnetic showers in tower 9 are more subject to leakage into the hadronic calorimeter. The electron is also not allowed to be in the region of the calorimeter where cryogenic connections to the solenoidal magnet are provided as it is an uninstrumented region of the detector. The electron shower is also required to be within an instrumented region of the CES. The local $z$ coordinate of the electron measured in the CES $\left(z_{\mathrm{CES}}\right)$ is not allowed to be in regions where the two halves of the calorimeter meet, $\left|z_{\mathrm{CES}}\right|<12 \mathrm{~cm}(<9 \mathrm{~cm}$ for $\mathrm{W}$ mass analysis). For the $\mathrm{W}$ mass analysis an additional cut is made, $\left|z_{\mathrm{CES}}\right|<230 \mathrm{~cm}$, to ensure the electron is away from the boundary between the electromagnetic and hadronic calorimeters where there is more chance of leakage into the hadronic compartment. The electromagnetic shower is also required to be fully contained within the active regions of the calorimeter wedge, away from the edges. The local $x$ coordinate of the cluster $\left(x_{\mathrm{CES}}\right)$ is required to be $\left|x_{\mathrm{CES}}\right|<18 \mathrm{~cm}$.

Additional kinematic and electron identification variables are defined as follows: 
- $E_{\mathrm{T}}$ : the transverse energy of the electron as measured in the electromagnetic calorimeter. The $\mathrm{W}$ width analysis requires the electron to have $E_{\mathrm{T}}$ greater than 30 $\mathrm{GeV}$ and the $\mathrm{W}$ mass analysis requires the electron $E_{\mathrm{T}}$ to be between $30 \mathrm{GeV}$ and $65 \mathrm{GeV}$. This narrower kinematic region ensures a well understood sample and the lower limit is also consistent with the previous $\mathrm{W}$ mass measurement.

- $E / p$ : the ratio of the transverse energy deposited in the calorimeter to the transverse momentum of the electron track as measured in the COT. This ratio is required to be in the region 0.8 and 1.3 for the $\mathrm{W}$ width analysis and less than 2.0 for the $\mathrm{W}$ mass analysis, again consistent with the previous measurement.

- $E_{\text {had }} / E_{\text {em }}$ : the ratio of the electron energy in the hadronic calorimeter to that in the electromagnetic calorimeter. Electrons deposit most of their energy in the electromagnetic calorimeter so the $E_{\text {had }} / E_{\text {em }}$ ratio is required to be less than 0.07 for the $\mathrm{W}$ width analysis and less than 0.1 for the $\mathrm{W}$ mass analysis so as to be consistent with the previous $\mathrm{W}$ mass measurement.

- $L_{\mathrm{shr}}$ : the variable quantifies the comparison between the lateral shower profile for the electron to the lateral shower profile for electrons from test beam data. This is required to be less than 0.3 for electromagnetic clusters to be consistent with an electron.

- $\Delta z$ : the distance in the $r-z$ plane between the extrapolated COT track and the position of the CES cluster. This difference is required to be less than $8 \mathrm{~cm}$ for the $\mathrm{W}$ width analysis and $5 \mathrm{~cm}$ for the $\mathrm{W}$ mass analysis (consistent with [22]).

- $\Delta x$ : the distance in the $r-\phi$ plane between the extrapolated COT track and the position of the CES cluster. This difference is required to be less than $10 \mathrm{~cm}$ for the $\mathrm{W}$ width analysis, with no requirement made in the $\mathrm{W}$ mass analysis. 
The cuts used to select an electron are listed in Table 4.1 for the $\mathrm{W}$ mass and width analyses.

\begin{tabular}{|l|l|l|}
\hline Variable & Cut value: W Width & Cut value: W Mass \\
\hline \hline$E_{\mathrm{T}}$ & $>25 \mathrm{GeV}$ & $>30 \mathrm{GeV},<65 \mathrm{GeV}$ \\
$p_{T}^{\mathrm{BC}}$ & $>10 \mathrm{GeV}$ & $>18 \mathrm{GeV}$ \\
$E / p$ & $>0.8,<1.3$ & $>0.0,<2.0$ \\
$E_{\mathrm{had}} / E_{\mathrm{em}}$ & $<0.07$ & $<0.1$ \\
$\left|z_{0}\right|$ & $<60 \mathrm{~cm}$ & $<60 \mathrm{~cm}$ \\
$|\Delta z|$ & $<8 \mathrm{~cm}$ & $<5 \mathrm{~cm}$ \\
$|\Delta x|$ & $<10 \mathrm{~cm}$ & - \\
$L_{\text {shr }}$ & $<0.3$ & $<0.3$ \\
$N_{\text {seg }}^{\text {axial }}$ & $\geq 3$ with $\geq 7 \mathrm{hits}$ & $\geq 3$ with $\geq 7 \mathrm{hits}$ \\
$N_{\text {seg }}^{\text {stereo }}$ & $\geq 3$ with $\geq 7$ hits & $\geq 3$ with $\geq 7$ hits \\
\hline
\end{tabular}

Table 4.1: Selection criteria for electrons in the mass and width analyses.

\subsubsection{Electron Reconstruction}

An electron is identified using its energy deposits in the CEM. The electron showers in the calorimeter can extend more than one tower. The towers in the calorimeter are sorted in $E_{\mathrm{T}}$ and the highest $E_{\mathrm{T}}$ tower is identified as the seed tower. Neighbouring towers in $\eta$ with the highest $E_{\mathrm{T}}$ are considered for addition to the cluster. An electron cluster is therefore completely contained within a single $\phi$ wedge in the calorimeter.

\subsection{Muon Selection}

Two types of selection criteria are used to select muons, tight and loose. Muons selected using the tight criteria, 'tight' muons, are identified in the central trackers, calorimeters and the muon chambers whereas muons selected using the loose criteria, 'loose' muons, are identified in the central trackers and calorimeters only. Whereas $\mathrm{W} \rightarrow \mu \nu$ events require one 'tight' muon, $\mathrm{Z} \rightarrow \mu^{+} \mu^{-}$events require one 'tight' and one 'loose' muon.

The muon track is constrained to the beamspot and required to originate within 60 $\mathrm{cm}$ in $z$ of the centre of the detector. The track is required to traverse at least three axial 
and three stereo superlayers with a minimum of seven hits in each layer. Additionally, for tracks where the silicon detector is not operational, at least five hits are required in the first axial COT superlayer $\left(N_{\text {hits }}^{\text {axial1 }}\right)$. The transverse momentum of the beam constrained track is required to be greater than $25 \mathrm{GeV}$ for the $\mathrm{W}$ width analysis and greater than 30 $\mathrm{GeV}$ for the $\mathrm{W}$ mass analysis.

In addition, to ensure that a muon track passes through all eight superlayers of the COT, the exit radius of the muon track at the COT endplates $\left(R_{\mathrm{COT}}\right)$ is required to be less than $137 \mathrm{~cm}$. A quantitative comparison is made between the COT hits on the track and the track reconstructed using these hits and the $\chi_{\text {track }}^{2} /$ dof of the comparison is required to be less than 3 for tracks with silicon hits and less than 2 for tracks without silicon hits. The track is required to have silicon hits if the silicon detector is operating well. The impact parameter $\left(d_{0}\right)$ of the track in the $r-\phi$ plane is required to be less than $0.04 \mathrm{~cm}$ for tracks with silicon hits and $0.2 \mathrm{~cm}$ for tracks without silicon hits. In the W mass analysis, a $d_{0}$ requirement of $0.1 \mathrm{~cm}$ is made.

The muon is a minimum ionising particle and leaves small energy deposits in the electromagnetic and hadronic calorimeters. The amount of energy deposited in the electromagnetic $\left(E_{\mathrm{em}}\right)$ and hadronic $\left(E_{\mathrm{had}}\right)$ calorimeters is required to be consistent with that from a minimum ionising particle. In the $\mathrm{W}$ width analysis, $E_{\mathrm{em}}$ is required to be less than $2 \mathrm{GeV}$ and $E_{\text {had }}$ is required to be less than $\left(5.6+0.014 \times p_{\mathrm{T}}\right) \mathrm{GeV}$, where the dependence on the muon $p_{\mathrm{T}}$ ensures that the selection efficiency is $p_{\mathrm{T}}$ independent. In the $\mathrm{W}$ mass analysis, the $E_{\mathrm{em}}$ requirement is $2.0 \mathrm{GeV}$ for a muon $p_{\mathrm{T}}$ less than $100 \mathrm{GeV}$ and $(2.0+$ $\left.0.0115 \times\left(p_{\mathrm{T}}-100\right)\right) \mathrm{GeV}$ for $p_{\mathrm{T}}$ greater than $100 \mathrm{GeV}$. The $E_{\text {had }}$ requirement is $(6.0+$ $\left.0.028 \times\left(p_{\mathrm{T}}-100\right)\right) \mathrm{GeV}$. A higher $p_{\mathrm{T}}$ muon leaves more energy in the calorimeters and is therefore more likely to fail the $E_{\mathrm{em}}$ and $E_{\text {had }}$ cuts. Making the $E_{\mathrm{em}}$ and $E_{\text {had }}$ cuts $p_{\mathrm{T}}$ dependent ensures that their efficiency does not vary with muon $p_{\mathrm{T}}$.

The muon track in the COT is required to be well matched with the muon stubs in the muon chambers. The distance between the extrapolated track and the muon stub in 
the $r-\phi$ plane in the $\mathrm{CMP}\left(\Delta X_{\mathrm{CMP}}\right), \mathrm{CMU}\left(\Delta X_{\mathrm{CMU}}\right)$ and $\mathrm{CMX}\left(\Delta X_{\mathrm{CMX}}\right)$ is required to be less than $6 \mathrm{~cm}, 3 \mathrm{~cm}$ and $5 \mathrm{~cm}$ respectively. In addition, the muon stub is required to be in a well instrumented region in the three muon chambers, CMU, CMP and CMX.

The criteria used to select 'loose' muons is given in Table 4.2 and the additional cuts used to select 'tight' muons is given in Table 4.3 for the $\mathrm{W}$ mass and width analyses. 'Tight' muons are required to pass all the 'loose' requirements as well as the additional ones in Table 4.3.

\begin{tabular}{|l|l|l|}
\hline Variable & Cut value $:$ W Width & Cut value $:$ W Mass \\
\hline \hline$p_{T}^{\mathrm{BC}}$ & $>25 \mathrm{GeV}$ & $>30 \mathrm{GeV}$ and $<65 \mathrm{GeV}$ \\
$\left|z_{0}\right|$ & $<60 \mathrm{~cm}$ & $<60 \mathrm{~cm}$ \\
$\left|d_{0}\right|$ & $<0.04(0.2) \mathrm{cm}$ for silicon (no silicon) & $<0.1 \mathrm{~cm}$ \\
$\chi_{\text {track }}^{2} /$ dof & $<3.0(2.0)$ for silicon (no silicon) & $<3.0$ \\
$N_{\text {seg }}^{\text {axial }}$ & $\geq 3$ with $\geq 7$ hits per superlayer & $\geq 3$ with $\geq 7$ hits per superlayer \\
$N_{\text {seg }}^{\text {steo }}$ & $\geq 3$ with $\geq 7$ hits per superlayer & $\geq 3$ with $\geq 7$ hits per superlayer \\
$N_{\text {hits }}^{\text {axial1 }}$ & $\geq 5($ no silicon tracks only) & - \\
$R_{\mathrm{COT}}$ & $<137 \mathrm{~cm}$ & - \\
$E_{\mathrm{em}}$ & $<2.0 \mathrm{GeV}$ & $<2.0+0.0115 \times\left(p_{\mathrm{T}}-100\right) \mathrm{GeV}^{1}$ \\
$E_{\mathrm{had}}$ & $<5.6+0.014 \times p_{\mathrm{T}} \mathrm{GeV}$ & $<6.0+0.028 \times\left(p_{\mathrm{T}}-100\right) \mathrm{GeV}{ }^{1}$ \\
silicon hits & for runs where silicon is 'good' & - \\
\hline
\end{tabular}

Table 4.2: Selection criteria for 'loose' muons in the mass and the width analyses.

\begin{tabular}{|l|l|l|}
\hline Variable & Cut value : W Width & Cut value : W Mass \\
\hline \hline CMUP or CMX stub & true & true \\
$\left|\Delta X_{\mathrm{CMP}}\right|$ & $<6 \mathrm{~cm}$ & $<6 \mathrm{~cm}$ \\
$\left|\Delta X_{\mathrm{CMU}}\right|$ & $<3 \mathrm{~cm}$ & $<3 \mathrm{~cm}$ \\
$\left|\Delta X_{\mathrm{CMX}}\right|$ & $<5 \mathrm{~cm}$ & $<5 \mathrm{~cm}$ \\
\hline
\end{tabular}

Table 4.3: Additional selection criteria for 'tight' muons in the mass and the width analyses.

\subsection{Event Cuts}

For $\mathrm{W}$ candidate events, the missing transverse energy attributed to the neutrino is defined as

$$
\overrightarrow{E_{T}}=\left(\overrightarrow{E_{T}}+\vec{U}\right)
$$


where $\vec{U}$ is the recoil vector and is defined as a vector sum over the transverse energy in the calorimeter excluding energy associated with the charged lepton (described in detail in Chapter 7 ) and $\overrightarrow{E_{T}}$ represents the transverse energy measurement for electrons in $\mathrm{W} \rightarrow e \nu$ events and the transverse momentum measurement for muons in $\mathrm{W} \rightarrow \mu \nu$ events.

The same requirements are placed on $E_{T}$ as on the $E_{T}$ of the charged lepton in the event. The $E_{T}$ in the $\mathrm{W}$ width analysis is required to be greater than $25 \mathrm{GeV}$, whereas a narrower kinematic range of $30 \mathrm{GeV}$ to $65 \mathrm{GeV}$ is required for the $\mathrm{W}$ mass analysis to ensure a well understood event sample and so that the lower limit is consistent with [22]. As explained in the previous chapter, the mass and width of the $\mathrm{W}$ boson are extracted from different regions of the $M_{T}$ distribution. The tail of the $M_{T}$ distribution provides information on the $\mathrm{W}$ width and therefore the range $50 \mathrm{GeV}$ to $200 \mathrm{GeV}$ is required for the $\mathrm{W}$ width analysis whereas the region around the Jacobian peak is most sensitive to the $\mathrm{W}$ mass and therefore the range $60 \mathrm{GeV}$ to $100 \mathrm{GeV}$ is required for the $\mathrm{W}$ mass analysis. The recoil $(|\vec{U}|)$ is required to be less than $20 \mathrm{GeV}$ in the $\mathrm{W}$ width analysis and less than $15 \mathrm{GeV}$ in the $\mathrm{W}$ mass analysis, consistent with the previous $\mathrm{W}$ mass measurement. This cut reduces contamination from QCD events which tend to populate the high $U$ region and also limits the $p_{\mathrm{T}}$ of the $\mathrm{W}$ boson so that the Jacobian edge is preserved.

For $\mathrm{W}$ events, a ' $\mathrm{Z}$ veto' cut is applied to reduce background from $\mathrm{Z} \rightarrow l^{+} l^{-}$events, the cut vetoes the event if there is an additional high $p_{\mathrm{T}}$ track in the event. The cuts used to select $\mathrm{W}$ events are listed in Table 4.4.

\begin{tabular}{|l|l|l|}
\hline Variable & Cut value : W Width & Cut value : W Mass \\
\hline \hline$E_{T}$ & $>25 \mathrm{GeV}$ & $>30 \mathrm{GeV}$ and $<65 \mathrm{GeV}$ \\
$M_{T}$ & $>50 \mathrm{GeV}$ and $<200 \mathrm{GeV}$ & $>60 \mathrm{GeV}$ and $<100 \mathrm{GeV}$ \\
$|\vec{U}|$ & $<20 \mathrm{GeV}$ & $<15 \mathrm{GeV}$ \\
$Z$ Veto & yes & yes \\
\hline
\end{tabular}

Table 4.4: Event cuts for the $\mathrm{W} \rightarrow l \nu$ sample in the $\mathrm{W}$ mass and width analyses.

\footnotetext{
${ }^{1}$ The cut is $E_{\mathrm{em}}<2 \mathrm{GeV}$ for $p_{\mathrm{T}}<100 \mathrm{GeV}$ and that given in the table for $p_{\mathrm{T}}>100 \mathrm{GeV}$.
} 
For $\mathrm{Z}$ candidate events, the invariant mass of the lepton pair $\left(M_{\ell \ell}\right)$ is required to lie within the range $80<M_{\ell \ell}<100 \mathrm{GeV}$ for the $\mathrm{W}$ width analysis and $66<M_{\ell \ell}<116$ $\mathrm{GeV}$ for the $\mathrm{W}$ mass analysis. The recoil cut is the same as that applied for $\mathrm{W}$ events. Additionally, the transverse momentum of the $\mathrm{Z}$ boson is required to be less than $50 \mathrm{GeV}$ for the $\mathrm{W}$ width analysis and less than $30 \mathrm{GeV}$ for the $\mathrm{W}$ mass analysis. The two decay leptons are required to have charges of opposite sign.

The cuts used to select $\mathrm{Z}$ events are listed in Table 4.5.

\begin{tabular}{|l|l|l|}
\hline Variable & Cut value : W Width & Cut value $:$ W Mass \\
\hline \hline$M_{\ell \ell}$ & $>80 \mathrm{GeV}$ and $<100 \mathrm{GeV}$ & $>66 \mathrm{GeV}$ and $<116 \mathrm{GeV}$ \\
$|\vec{U}|$ & $<20 \mathrm{GeV}$ & $<15 \mathrm{GeV}$ \\
$p_{T}^{Z}$ & $<50 \mathrm{GeV}$ & $<30 \mathrm{GeV}$ \\
Opposite sign & yes & yes \\
\hline
\end{tabular}

Table 4.5: Event cuts for the $\mathrm{Z} \rightarrow l^{+} l^{-}$sample in the $\mathrm{W}$ mass and width analyses.

The event yields obtained from selecting $\mathrm{W}$ and $\mathrm{Z}$ events using the above described selection criteria are given for both analyses in Table 4.6. The yields for the electron and muon channels in the $\mathrm{W}$ width (W mass) analysis correspond to $370 \mathrm{pb}^{-1}\left(2400 \mathrm{pb}^{-1}\right)$ and $330 \mathrm{pb}^{-1}\left(2300 \mathrm{pb}^{-1}\right)$ of data respectively.

\begin{tabular}{|l|r|r|}
\hline Data sample & W Width $\left(350 \mathrm{pb}^{-1}\right)$ & W Mass $\left(2350 \mathrm{pb}^{-1}\right)$ \\
\hline \hline $\mathrm{Z} \rightarrow e^{+} e^{-}$ & 2,909 & 33,039 \\
$\mathrm{~W} \rightarrow e \nu$ & 127,432 & 706,967 \\
$\mathrm{Z} \rightarrow \mu^{+} \mu^{-}$ & 6,271 & 63,699 \\
$\mathrm{~W} \rightarrow \mu \nu$ & 108,808 & 666,228 \\
\hline
\end{tabular}

Table 4.6: Event yields for the event samples used in the W mass and width analyses. (The integrated luminosities given in brackets refer to the average integrated luminosity for the electron and muon decay channels). 


\section{Chapter 5}

\section{Event Generation}

The simulation of the production and subsequent decay of the $\mathrm{W}$ boson proceeds in two main steps. $\mathrm{W}$ and $\mathrm{Z}$ events are generated using a Monte Carlo event generator which uses a sequence of random numbers to simulate some of the particles produced in the interaction of the $p \bar{p}$ and their decay. The generator outputs a list of final state particles; electrons, positrons, photons and neutrinos and their true, unsmeared four-momenta. The second step of the event simulation involves propagating these generated particles through a detector simulation where particle interactions with the material of the detector, e.g bremsstrahlung is simulated and their energies and momenta are smeared to match those reconstructed in the sub-detectors. This chapter will describe the event generation process in the $\mathrm{W}$ width analysis in detail. This is identical to the $\mathrm{W}$ mass measurement apart from the treatment of QED. The systematics on the $\mathrm{W}$ width from the different aspects of the event generation will also be discussed.

A precision measurement of the mass or width of the $\mathrm{W}$ boson requires the inclusion of higher order corrections to the tree level diagram. The $\mathrm{W}$ production and decay mechanism receives corrections to the leading order diagram from two major next to leading order (NLO) contributions, QCD and QED. The contribution from NLO QCD is shown in Figure 5.1 where the $\mathrm{W}$ boson recoils against a gluon radiated from the incoming quark or antiquark giving it a finite transverse momentum. In addition to QCD corrections, there are QED effects arising from the emission of real and virtual photons from particles 
carrying electromagnetic charge, examples of which are shown in Figure 5.2. A generator combining the effects of NLO QCD and NLO QED at the precision required for the W mass and width analyses does not exist and a convolution of different generators that deal with specific higher order corrections are used to model the event generation process.

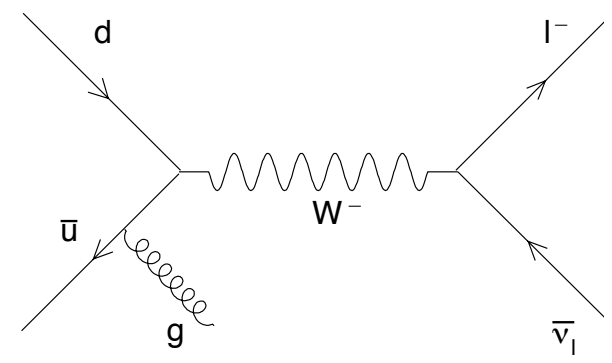

Figure 5.1: The production of a $\mathrm{W}$ boson with initial state gluon radiation.
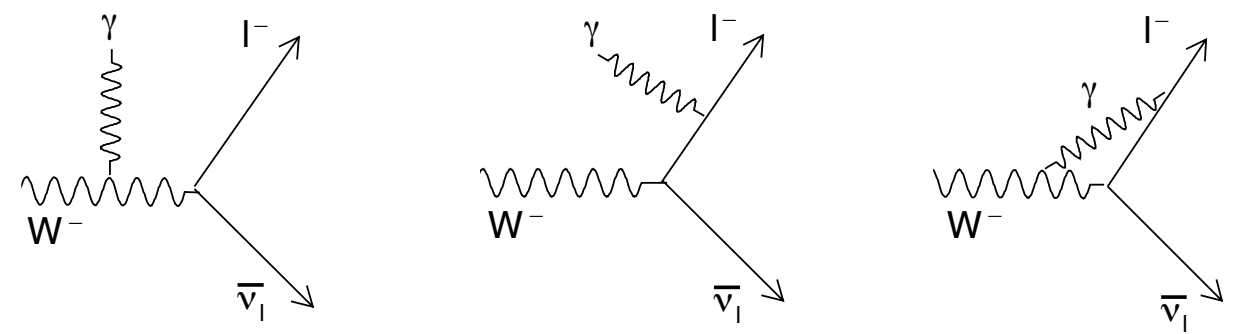

Figure 5.2: Feynman diagrams for the radiative corrections to $\mathrm{W}$ production and decay simulated by the Berends and Kleiss program.

A LO Monte Carlo event generator [42] interfaced with a PDF set is used to produce a set of final state particles where the flavour and longitudinal momenta of the quarks participating in the hard scatter are determined by PDFs. This produces a $\mathrm{W}$ boson with a longitudinal momentum but zero transverse momentum. It will be explained in this chapter how the transverse momentum of the $\mathrm{W}$ is simulated. This is particularly important for the simulation of the recoil since the boson transverse momentum is one of the inputs into the model used to parameterise the recoil. The corrections to the W 
production and decay process from higher order QED and electroweak contributions will also be discussed.

\subsection{Parton Distribution Functions (PDF)}

The production of a $\mathrm{W}$ boson at the Tevatron is dominated by the annihilation of a valence quark from the proton and a valence antiquark from the antiproton. The probability of finding a parton of a certain type carrying a fraction $x$ of the proton or antiproton's momentum is provided by the parton distribution functions. The momenta of the interacting partons determine the longitudinal momentum of the $\mathrm{W}$ boson. Whereas the transverse mass is invariant under longitudinal boosts of the $\mathrm{W}$, the incomplete rapidity coverage of the detectors results in a dependence of the measured $M_{T}$ distribution on the longitudinal momentum through cuts on the detector acceptance and cuts on the kinematics of the decay leptons. Uncertainties on PDFs therefore contribute as one of the theoretical uncertainties on the measured mass and width of the $\mathrm{W}$ boson.

Two major collaborations, namely CTEQ [45] and MRST [46], independently perform global fits to data and provide parton distribution functions. The CTEQ collaboration allows 20 free parameters to vary in the fit and uses the Hessian matrix method to estimate the PDF uncertainty. Twenty orthogonal eigenvector directions are determined, from which the collaboration provides 40 PDFs, corresponding to a $\pm 90 \%$ confidence level. The MRST collaboration allows 15 free parameters in their model, providing 30 PDF sets.

The following formula is used to compute the uncertainty due to PDFs on $\Gamma_{W}$ and $M_{W}$

$$
\Delta X_{W}=\frac{1}{2} \sqrt{\sum_{i=1}^{N}\left[\Delta X_{W}\left(S_{i}^{+}\right)-\Delta X_{W}\left(S_{i}^{-}\right)\right]^{2}}
$$

where $X=M_{W}, \Gamma_{W}$ and $\Delta X_{W}\left(S_{i}^{ \pm}\right)$corresponds to a shift in the fitted value of $X$ between the central PDF error set and the PDF eigenvector $S_{i}$ for the $\pm 90 \%$ shifts.

Each of these 40 (30) PDF error sets provided by CTEQ (MRST) is used to produce a 
$M_{T}$ distribution which is then fitted to pseudo-data simulated using the central PDF set to obtain a shift in the width for each of the eigenvectors. The results of the fit, shown in Figure 5.3 for the CTEQ PDF, are used to obtain $\Delta \Gamma_{W}$ in the above equation.

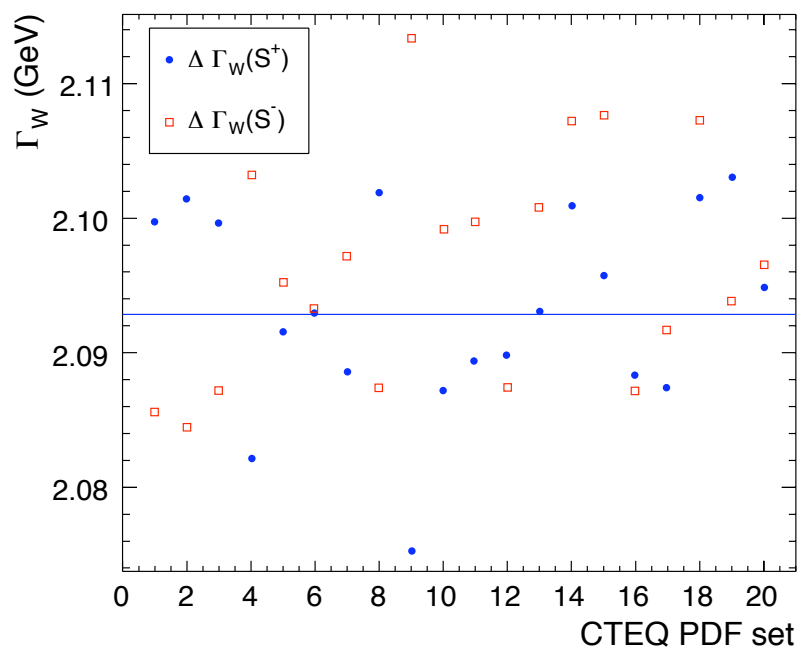

Figure 5.3: Fitted $\Gamma_{W}$ for CTEQ PDF set where $\Delta \Gamma_{W}\left(S^{ \pm}\right)$represents the shift in the width due to varying the eigenvectors by $\pm 90 \%$. The horizontal line is the value of $\Gamma_{W}$ obtained for the central PDF set.

The CTEQ PDF sets 1 and 3 in Figure 5.3 are dominated by the u-valence and dvalence quarks and eigenvector 9 which gives the largest uncertainty on the width is not dominated by any particular parameter.

The systematic uncertainty on the $\mathrm{W}$ width from the CTEQ and MRST PDFs for the electron and muon channels is shown in Table 5.1. The MRST error is lower than the CTEQ error and as a conservative estimate of the uncertainty, the CTEQ error is used. Since the CTEQ and MRST PDF sets correspond to a $90 \%$ probability, the uncertainties are divided by a factor of 1.6 to obtain the $1 \sigma$ uncertainty.

\begin{tabular}{|l|cc|}
\hline & $\Delta \Gamma_{W}:$ CTEQ & $\Delta \Gamma_{W}:$ MRST \\
\hline \hline Electron channel & $16 \mathrm{MeV}$ & $11 \mathrm{MeV}$ \\
Muon channel & $16 \mathrm{MeV}$ & $11 \mathrm{MeV}$ \\
\hline
\end{tabular}

Table 5.1: Systematic uncertainties on $\Gamma_{W}$ due to $1 \sigma$ uncertainty in the CTEQ and MRST PDF sets. 
The MRST and CTEQ PDF sets used to obtain the PDF systematic uncertainty are calculated to NLO. Additional theoretical uncertainties can arise from highers orders in perturbation theory. This uncertainty can be estimated by using the MRST PDF which provides both NLO and NNLO PDFs. The shift in the W width obtained by fitting using the NLO and NNLO PDFs is found to be $12 \mathrm{MeV}$ for both the electron and muon channels. This is added in quadrature with the PDF uncertainties shown in Table 5.1.

\section{$5.2 \quad$ Boson $p_{\mathrm{T}}$}

Quarks and antiquarks from the incoming proton and antiproton emit gluons. W and Z bosons produced in the interaction recoil against this initial state gluon radiation, acquiring a transverse momentum equal and opposite to it. Whereas the $p_{\mathrm{T}}$ distribution of the $\mathrm{Z}$ boson $\left(p_{T}^{Z}\right)$ is well reconstructed from the decay leptons, the $\mathrm{W}$ boson $p_{\mathrm{T}}\left(p_{T}^{W}\right)$ is not well determined due to the neutrino escaping the detector. The $\mathrm{Z} \rightarrow l^{+} l^{-}$samples are therefore used to constrain the form of the $\mathrm{Z} p_{\mathrm{T}}$. A precise theoretical calculation is then used to obtain the $\mathrm{W} p_{\mathrm{T}}$ distribution from the $\mathrm{Z} p_{\mathrm{T}}$.

\subsubsection{Determination of $p_{T}^{Z}$ and $p_{T}^{W}$}

The $p_{T}^{Z}$ distribution is simulated by using a NLO QCD calculation [49] that matches perturbative $\mathrm{QCD}$ at high $p_{\mathrm{T}}$ to a gluon resummation formalism [52] at low $p_{\mathrm{T}}$. The resummation at low $p_{\mathrm{T}}$ has contributions from low $p_{\mathrm{T}}$ gluons and therefore has a significant non-perturbative component that has to be parameterised by fitting to data. The Brock-Landry-Nadolsky-Yuan (BLNY) [51] parameterisation is used in the resummation calculation. The BLNY functional form has 4 free parameters; $g_{1}, g_{2}, g_{3}$ and $b_{\max }$, the values of which are obtained by fitting to data from fixed target low $p_{\mathrm{T}}$ Drell-Yan experiments and Tevatron Run 1 experiments. Of the 4 free parameters, $g_{2}$ is most sensitive to the $p_{\mathrm{T}}$ distribution at high $\sqrt{\hat{s}}$. The BLNY form is used to fit to the $p_{\mathrm{T}}$ distribution in $\mathrm{Z} \rightarrow l^{+} l^{-}$events allowing the parameter $g_{2}$ to vary whilst fixing the other parameters to 
their global fit values given in [49]. The $p_{T}^{Z}$ in $\mathrm{Z} \rightarrow e^{+} e^{-}$and $\mathrm{Z} \rightarrow \mu^{+} \mu^{-}$events is fitted separately to obtain a best fit $g_{2}$

$$
\begin{aligned}
& g_{2}=(0.62 \pm 0.08) \mathrm{GeV}^{2}, \quad \chi^{2} / \text { dof }=51 / 47 \quad\left(\mathrm{Z} \rightarrow e^{+} e^{-}\right) \\
& g_{2}=(0.68 \pm 0.05) \mathrm{GeV}^{2}, \quad \chi^{2} / \text { dof }=52 / 47 \quad\left(\mathrm{Z} \rightarrow \mu^{+} \mu^{-}\right)
\end{aligned}
$$

The fitted values of $g_{2}$ for the electron and muon channel are consistent with each other and therefore a combined fit is performed to the $p_{T}^{Z}$ distribution in both samples simultaneously. The value of $g_{2}$ obtained for the combined fit is

$$
g_{2}^{e+\mu}=(0.66 \pm 0.04) \mathrm{GeV}^{2}, \quad \chi^{2} / \text { dof }=103 / 96 .
$$

The results of the fits to the $p_{\mathrm{T}}$ distribution in the $\mathrm{Z} \rightarrow e^{+} e^{-}$and $\mathrm{Z} \rightarrow \mu^{+} \mu^{-}$channels are shown in Figure 5.4.

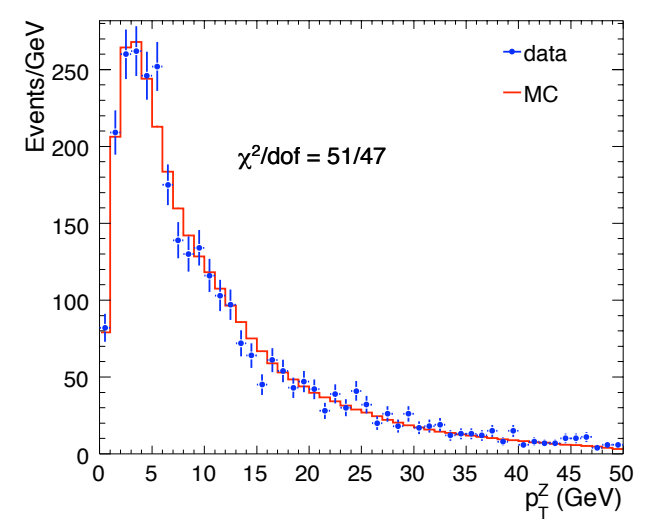

(a)

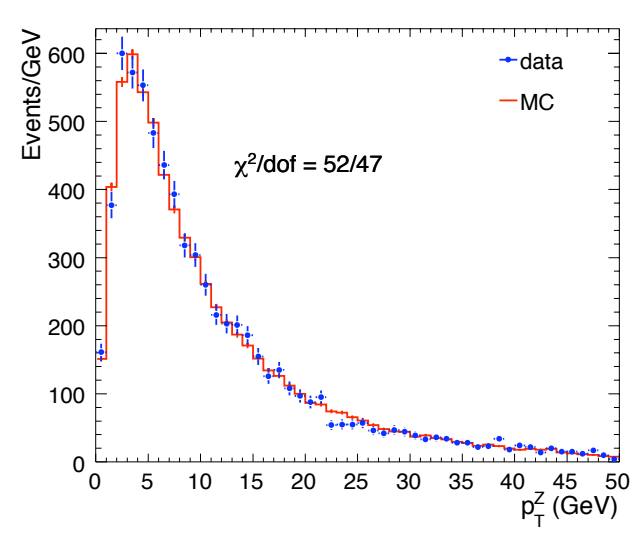

(b)

Figure 5.4: The measured $p_{T}^{Z}$ distributions compared to the best fit $\mathrm{MC}$ prediction for (a) $\mathrm{Z} \rightarrow e^{+} e^{-}$ data and (b) $\mathrm{Z} \rightarrow \mu^{+} \mu^{-}$data.

The average absolute rapidity of the $\mathrm{Z}$ boson used in the fit is 0.3 , however, the events in the simulation are generated differential in both $p_{\mathrm{T}}$ and rapidity. The $p_{\mathrm{T}}$ distribution in the simulation is therefore reweighted using a function taken from the theoretical calculation of $\frac{d \sigma}{d p_{T}}(|Y|=0.3) / \frac{d^{2} \sigma}{d Y d p_{T}}(|Y|=Y)$, where $Y$ is the boson rapidity. 
The $\mathrm{W} p_{\mathrm{T}}$ distribution is obtained by reweighting the $\mathrm{Z} p_{\mathrm{T}}$ distribution which is differential in $p_{\mathrm{T}}$ and rapidity to a $p_{T}^{W}$ distribution differential in $p_{\mathrm{T}}$, rapidity and $\hat{s}$ using a theoretical calculation of $\frac{d^{3} \sigma}{d Y d p_{T} d \hat{s}}(W) / \frac{d^{2} \sigma}{d Y d p_{T}}(Z)[53]$.

\subsection{2 $\quad p_{T}^{W}$ Systematic}

The shape of the $p_{\mathrm{T}}$ distribution is determined at low $p_{\mathrm{T}}$ by the BLNY functional form with $g_{2}$ tuned to CDF data as shown above and at high $p_{\mathrm{T}}$ by the perturbative QCD calculation. An uncertainty on this shape could be obtained by varying the 4 BLNY parameters by their errors, however, since these govern the low $p_{\mathrm{T}}$ region, the uncertainty obtained by this method may not cover the entire $p_{\mathrm{T}}$ distribution. The shape of the $p_{\mathrm{T}}$ distribution is varied according to

$$
f\left(B, p_{T}\right)=1+B \times p_{T}
$$

such that

$$
\left(\frac{d \sigma}{d p_{T}}\right)_{\text {total }}=\left(\frac{d \sigma}{d p_{T}}\right)_{\mathrm{NLO}} \times f\left(B, p_{T}\right)
$$

where $\left(\frac{d \sigma}{d p_{T}}\right)_{\mathrm{NLO}}$ is the $p_{\mathrm{T}}$ distribution obtained using the best fit $g_{2}$ mentioned in the previous section and $B$ is a factor to be determined that distorts the shape at high $p_{\mathrm{T}}$.

A two-dimensional fit is performed to obtain $g_{2}$ and $B$. The best fit values obtained were

$$
g_{2}=(0.64 \pm 0.04) \mathrm{GeV}^{2}, B=(-0.001 \pm 0.001) \mathrm{GeV}^{-1}
$$

and the $g_{2}-B$ covariance matrix is found to be

$$
\left(\begin{array}{cc}
0.002 & -1.38 \times 10^{-7} \\
-1.38 \times 10^{-7} & 1.0 \times 10^{-6}
\end{array}\right) \text {. }
$$

This covariance matrix is sampled to obtain the $1 \sigma$ and $2 \sigma$ correlation contours shown in Figure 5.5. 


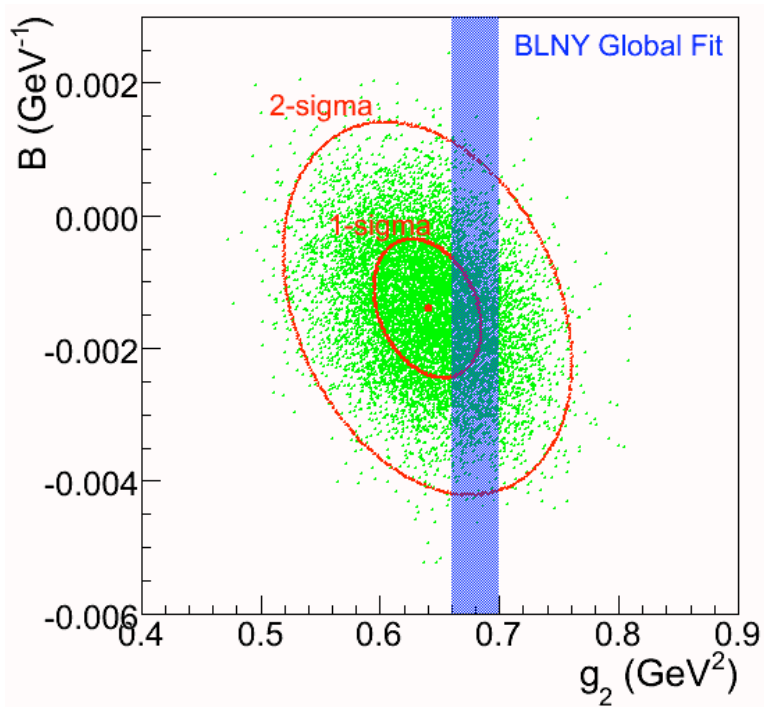

Figure 5.5: The $1 \sigma$ and $2 \sigma$ correlation contours for $g_{2}$ and $B$ obtained by sampling the covariance matrix. The BLNY global fit value of $g_{2}$ is superimposed.

The value for $g_{2}$ is consistent with the combined fit value $g_{2}{ }^{e+\mu}$ and the distortion factor $B$ is consistent with zero. The uncertainty arising from $g_{2}-B$ is obtained by sampling the covariance matrix to obtain values for these parameters which are then input in the simulation to create $M_{T}$ distributions. Simulation templates of $M_{T}$ created with different input $\mathrm{W}$ width values are used to fit to the $M_{T}$ distributions to obtain a Gaussian distribution of the fitted $\Gamma_{W}$. The uncertainty $\left(\Delta \Gamma_{W}^{g_{2} B}\right)$ attributed to the parameters $g_{2}$ and $B$ is taken from the width of the Gaussian.

The uncertainty on the $p_{\mathrm{T}}$ distribution from the other BLNY parameters, $g_{1}$ and $g_{3}$ is obtained by varying them by their $95 \%$ confidence level uncertainty. The uncertainties arising from these 2 parameters are denoted as $\Delta \Gamma_{W}^{g_{1}}$ and $\Delta \Gamma_{W}^{g_{3}}$.

The ratio used to reweight the $p_{\mathrm{T}}$ distribution to the correct rapidity and the theoretical ratio $\frac{d^{3} \sigma}{d Y d p_{T} d \hat{s}}(W) / \frac{d^{2} \sigma}{d Y d p_{T}}(Z)$ used to obtain $p_{T}^{W}$ from $p_{T}^{Z}$ have a negligible theoretical uncertainty. The systematic uncertainty on the $\mathrm{W}$ width from other effects such as the dependence on PDFs and the value of $\alpha_{s}$ is found to be $2 \mathrm{MeV}$. The systematic uncertainties for all the individual contributions and the total $p_{T}^{W}$ systematics obtained by adding 
the various contributions in quadrature is given in Table 5.2.

\begin{tabular}{|cccc|c|}
\hline$\Delta \Gamma_{\mathrm{W}}^{g_{1}}$ & $\Delta \Gamma_{\mathrm{W}}^{g_{2}, \mathrm{~B}}$ & $\Delta \Gamma_{\mathrm{W}}^{g_{3}}$ & $\Delta \Gamma_{\mathrm{W}}^{\text {other }}$ & $\Delta \Gamma_{\mathrm{W}}[$ total $]$ \\
\hline \hline $2 \mathrm{MeV}$ & $6 \mathrm{MeV}$ & $2 \mathrm{MeV}$ & $2 \mathrm{MeV}$ & $7 \mathrm{MeV}$ \\
\hline
\end{tabular}

Table 5.2: The $p_{T}^{W}$ systematic from the individual contributions and the total systematic. The error quoted is $100 \%$ correlated between the electron and muon decay channels.

\subsection{QED}

In the $\mathrm{W}$ boson production and decay process, photons can be radiated from the initial quarks, the $\mathrm{W}$ boson and the final state charged lepton. The dominant contribution to the QED correction is photon radiation from the final state charged lepton.

A photon radiated from an electron carries away some fraction of its energy, thereby reducing the electron transverse momentum $\left(p_{T}\right)$ and energy $\left(E_{T}\right)$. The transverse energy of the electron is measured from its electromagnetic shower in the calorimeter. If the photon is emitted at a small angle to the electron, its shower in the calorimeter overlaps with the electron shower and it is effectively included in the electron $E_{T}$ measurement. A photon emitted collinear with the electron therefore does not have a significant impact on the $M_{T}$ distribution. However, if the photon is emitted at a wide angle to the electron, its energy is not recombined with the electron energy and gives a measured $E_{T}$ that is low resulting in a $M_{T}$ distribution that is skewed towards lower mass values. The same is true for the invariant mass distribution in $\mathrm{Z} \rightarrow e^{+} e^{-}$events where both electrons can radiate photons and neglecting QED effects results in a lower measured $E_{T}$ for the electrons and thus a lower invariant mass for the $\mathrm{Z}$ boson.

Whereas muons radiate at a lower rate than electrons, neglecting photon emission from muons has a more pronounced effect than that for electrons. This is because the quantity of interest for muons is the transverse momentum, since they leave little energy in the 
calorimeter for their $E_{T}$ to be measured. The muon $p_{T}$ is more affected by the emission of photons as the transverse momentum carried away by the photons is not recovered.

QED effects can therefore have a large impact on the $M_{T}$ distribution and hence the extracted width of the $\mathrm{W}$ boson and need to be simulated accurately. The QED corrections to $\mathrm{W}$ and $\mathrm{Z}$ production and decay are simulated using the Berends and Kleiss program [43] which was interfaced with the event generator. The program includes real and virtual QED corrections and can generate up to one photon from the charged lepton and the half of the $\mathrm{W}$ propagator that contributes to final state radiation (photon emission from the $\mathrm{W}$ also contributes to initial state radiation) as shown in Figure 5.2.

An estimation of the systematic uncertainty from QED can be determined by considering higher order QED corrections, such as the emission of two photons. This is done by utilising the PHOTOS [48] program which can generate up to two photons from the charged lepton. The algorithm uses the leading log approximation (LLA) and is therefore accurate in the soft and collinear emission limits only. As noted earlier, QED radiation from the charged lepton affects the $M_{T}$ distribution in $\mathrm{W}$ events and the invariant mass distribution in $\mathrm{Z}$ events. Fits are performed to the $\mathrm{Z}$ invariant mass distribution to obtain the calorimeter and tracker response and resolution as described in Chapter 6. If QED effects are neglected, the invariant mass distribution is skewed towards lower values and this leads to shifts in the calorimeter and tracking scales and resolutions. Since these extracted scales and resolutions are subsequently applied to W events, they can indirectly affect the $M_{T}$ distribution. The estimation of a systematic must therefore take into account both the direct and indirect effects. The shifts on the $\mathrm{W}$ width from the direct effect on the $M_{T}$ of the second photon and the indirect effects from the shifts in the calorimeter and tracking scales and resolutions are $100 \%$ correlated and shown in Table 5.3. 


\begin{tabular}{|l|ccc|c|}
\hline & $\Delta \Gamma_{W}$ (fit) & $\Delta \Gamma_{W}$ (scale) & $\Delta \Gamma_{W}$ (resolution) & $\Delta \Gamma_{W}$ (total) \\
\hline \hline Electron channel & $-9 \mathrm{MeV}$ & $+5 \mathrm{MeV}$ & $-4 \mathrm{MeV}$ & $-8 \mathrm{MeV}$ \\
Muon channel & $-7 \mathrm{MeV}$ & $+16 \mathrm{MeV}$ & $-10 \mathrm{MeV}$ & $1 \mathrm{MeV}$ \\
\hline
\end{tabular}

Table 5.3: Systematic uncertainties on $\Gamma_{W}$ due to QED for the electron and muon decay channels, where $\Delta \Gamma_{W}$ (fit) represents the shift in the W width from the second photon, $\Delta \Gamma_{W}$ (scale) and $\Delta \Gamma_{W}$ (resolution) are the shifts in the width from shifts in the calorimeter scale and resolution for the electron channel and the momentum scale and resolution for the muon channel. $\Delta \Gamma_{W}$ (total) represents the total shift in the width.

\subsection{Electroweak Box Diagrams}

Electroweak corrections from non-resonant contributions to the $p \bar{p} \rightarrow l \nu$ process arise predominantly from the $W / Z$ box diagrams shown in Figure 5.6. These diagrams have a nonnegligible contribution in the high $M_{T}$ region [56] and can therefore affect the measured $\Gamma_{W}$. The magnitude of this effect is estimated by using the WGRAD [56] program which includes all NLO electroweak corrections. WGRAD is used together with a simplified detector model, where the lepton and $E_{T}$ resolutions are described by a simple Gaussian. The effect on the width is obtained by using WGRAD with and without the non-resonant contribution. A ratio of the $M_{T}$ distribution with and without the non-resonant contribution is obtained and used to reweight a pseudo-data $M_{T}$ histogram. Simulation templates of the nominal $M_{T}$ distribution with different input widths are fitted to this reweighted histogram to determine the shift in the measured width that would be obtained by neglecting non-resonant box diagrams. The result is shown in Table 5.4. The final value of the width is corrected for this shift. The systematic uncertainty on this is obtained by varying the $E_{T}$ smearing in the simplified detector model so that it represents the $E_{T}$ resolution uncertainty obtained from fits to data. The systematic is given in Table 5.4.

\begin{tabular}{|l|cc|}
\hline & $\Gamma_{W}$ correction & $\Delta \Gamma_{W}$ \\
\hline \hline Electron channel & $+11 \mathrm{MeV}$ & $6 \mathrm{MeV}$ \\
Muon channel & $+12 \mathrm{MeV}$ & $6 \mathrm{MeV}$ \\
\hline
\end{tabular}

Table 5.4: The shift in $\Gamma_{W}$ due to non-resonant electroweak corrections for the electron and muon decay channels. The uncertainty on the shift comes from varying the $\not_{T}$ resolution. 

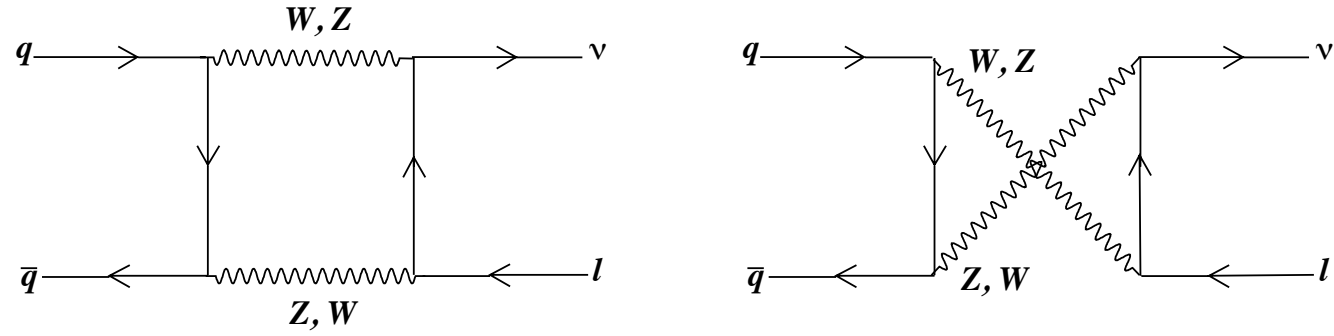

Figure 5.6: Feynman diagrams for the W, $\mathrm{Z}$ box diagrams in the $p \bar{p} \rightarrow l \nu$ process. 


\section{Chapter 6}

\section{Event Simulation}

Electrons, muons and photons generated by the event generator must be propagated through a simulation that incorporates the effects of the detector on the measured particle properties such that they match those measured in the data. A detailed GEANT-3 [60] ${ }^{1}$ simulation is extensively used by the CDF collaboration (CdfSim). CdfSim traces every particle produced in an interaction through the detector and uses the full detector geometry and material properties to account for energy losses in the material. This process is repeated for every particle and is rather slow.

The $\mathrm{W}$ width measurement requires a detailed study of systematics affecting the transverse mass distribution and this is done by utilising simulation templates of $M_{T}$ which must be produced with enough events for the statistical fluctuations to be negligible. Simulation templates of the $M_{T}$ distribution with different input $\mathrm{W}$ widths are used throughout the analysis to estimate systematics and a fast and tunable simulation is developed that will allow these studies to be carried out efficiently and for different aspects of the simulation to be tuned to the precision required by the measurement. The fast simulation incorporates the processes and interactions of the particles with the material of the sub-detector they are traversing. It simulates the various sub-detectors using a combination of input from CdfSim and parameterisations obtained from fitting to data thus making it faster than propagating the particles through a full detector simulation.

\footnotetext{
${ }^{1}$ The GEANT-3 simulation is based on measurements of the material carried out during detector construction and tuned to Run II data sample of electrons from photon conversions.
} 
This chapter will describe the fast simulation in detail. It will start from the interaction point and step through each sub-detector explaining the interactions of the particles with the materials of the detector and the methods used to determine their transverse energy and momenta.

The sub-detectors traversed by each of the particles produced in the $\mathrm{W}$ decay are shown in Figure 6.1. An electron, for example, travelling on its way outwards from the

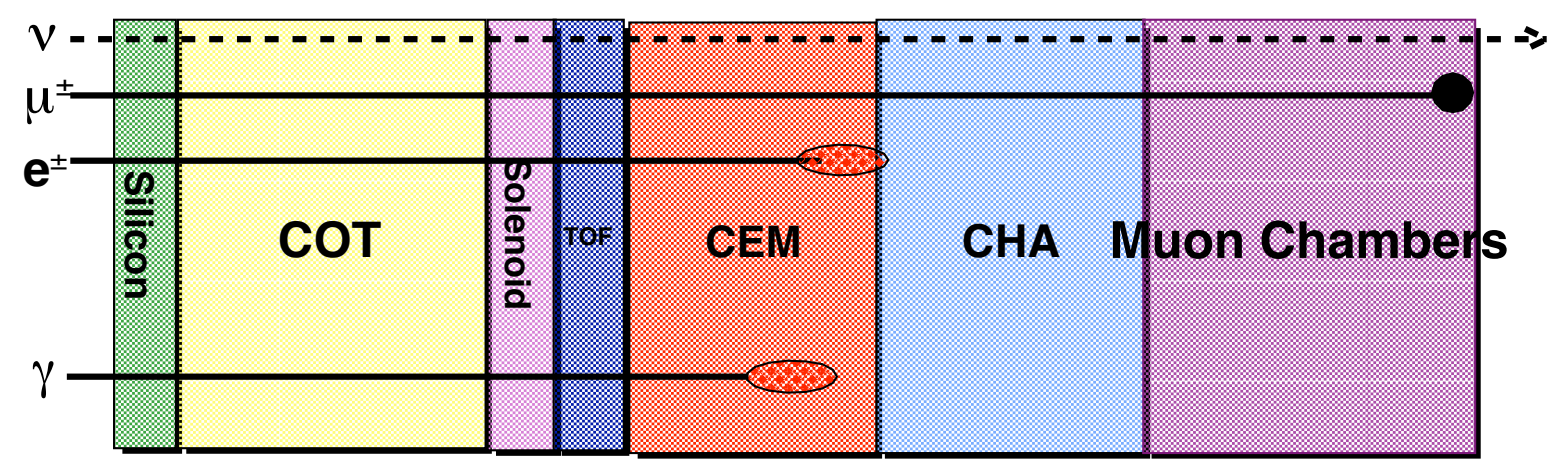

Figure 6.1: Schematic diagram showing the sub-detectors traversed by particles at CDF.

interaction point first comes across the silicon detector and loses energy by various mechanisms including bremsstrahlung before entering the COT, where the curvature of the track is used to determine its momentum and charge. Some more energy is subsequently lost in the solenoid and time-of-flight detectors before the electron reaches the calorimeters, where it deposits most of its energy in the CEM, with some leakage into the hadronic calorimeter. This chapter will explain how these effects are modelled in the simulation. 


\subsection{Silicon}

All particles from the interaction point first encounter the silicon detector which presents a large amount of passive material. Accurate determination of the amount and type of material is needed to evaluate the amount of energy lost by particles in the silicon tracking volume. This is achieved using SiliMap [62], a program which provides a description of the silicon tracker in the form of a binned map of the passive material properties obtained by scanning the full detector geometry as implemented in the GEANT-3 based CdfSim. The material description is implemented as a finely binned 3-dimensional look-up table storing the following material properties; the number of radiation lengths, $X_{0}$, the normalisation constant $K Z / A$ and the ionisation constant $I$ as a function of radius, azimuthal angle and the $z$ direction for each of the 32 SiliMap radial layers. The material properties from SiliMap are used to determine the energy lost by muons, electrons and photons in the silicon tracker volume.

\section{Muons}

The dominant energy loss mechanism for muons in the silicon detector is ionisation. The mean rate of energy lost by a muon is simulated using the Bethe-Bloch equation [57]

$$
-\frac{d E}{d x}=\frac{K}{\beta^{2}}\left[\frac{1}{2} \ln \frac{2 m_{e} c^{2} \beta^{2} \gamma^{2} T_{\max }}{I^{2}}-\beta^{2}-\frac{\delta}{2}\right]
$$

where $K$ is an overall constant factor, $I$ is the ionisation potential, $T_{\max }$ is the maximum kinetic energy that can be given to a free electron in a single collision and $\delta$ is the density effect correction. The factors $K$ and $I$ are taken from SiliMap, whereas the density effect correction is calculated using Sternheimer's parameterisation [58] assuming the material traversed in silicon.

\section{Electrons}


The dominant energy loss mode for electrons in the silicon detector is bremsstrahlung. The probability for photon radiation in a material layer is given by

$$
P_{\gamma}=d X_{0} \times \frac{4}{3}\left[\ln \left(y_{\max } / y_{\min }\right)-\left(y_{\max }-y_{\min }\right)+\frac{3}{8}\left(y_{\max }-y_{\min }\right)^{2}\right]
$$

where $y_{\min }=0.001$ and $y_{\max }=1.0$ are the minimum and maximum fraction of the electron's energy transferred to the radiated photon respectively. The fractional radiation length traversed, $d X_{0}$, is provided by SiliMap. The energy fraction, $y$, is distributed according to

$$
\frac{d \sigma}{d y}=\frac{1}{y}\left(\frac{4}{3}-\frac{4 y}{3}+y^{2}\right)
$$

Ionisation energy loss is also simulated for electrons in the silicon detector.

\section{Photons}

Photons lose energy in the silicon detector predominantly via pair production. The high energy limit for the photon conversion probability in a thin material layer is given by

$$
P_{e^{+} e^{-}}=(7 / 9) d X_{0}
$$

where the fractional radiation length traversed, $d X_{0}$, is again provided by SiliMap. The differential cross-section is [23]

$$
\frac{d \sigma}{d x}=1-\frac{4}{3} x(1-x)
$$

where $x$ is the fractional energy transfer to the pair-produced electron.

\subsubsection{Systematic Uncertainty}

The interactions of particles with matter in the simulation proceeds iteratively, where the initial electron from $\mathrm{W}$ decay emits a bremsstrahlung photon which may convert to an $e^{+} e^{-}$pair that may subsequently produce more bremsstrahlung photons and so on. The fast simulation simulates only two iterations by default, as shown in Figure 6.2 for 
$\mathrm{Z} \rightarrow e^{+} e^{-}$decay. The process stops when the bremsstrahlung photons from the initial electron(s) convert, with the conversion electrons not allowed to emit further photons. In order to determine the uncertainty from only allowing two iterations, the simulation is setup to produce up to three and four iterations and a maximum shift in the fitted width value of $8 \mathrm{MeV}$ is found.

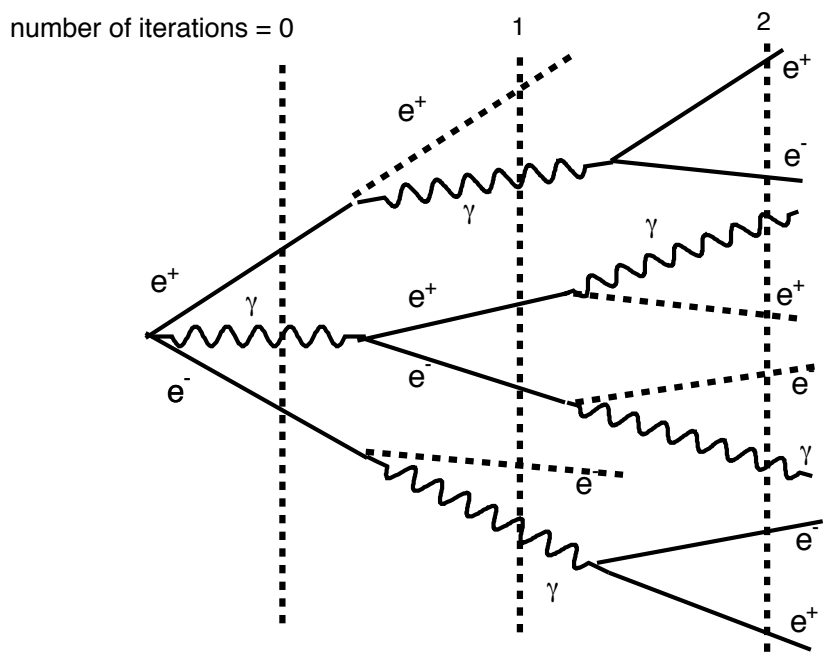

Figure 6.2: The evolution of an electromagnetic shower in $\mathrm{Z} \rightarrow e^{+} e^{-}$events, with electrons producing photons by bremsstrahlung and photons converting to $e^{+} e^{-}$pairs. Two iterations are simulated by the fast simulation.

Bremsstrahlung radiation in the silicon tracker is simulated for $y$ above 0.001 . However, the cross-section for the emission of bremsstrahlung photons is suppressed below a cut-off due to interference caused by multiple scattering. This is known as the Migdal effect and the suppression occurs at $y_{\min } \sim E_{e} /(72 \mathrm{TeV})$ in silicon, where $E_{e}$ is the incident electron energy in $\mathrm{GeV}$. Therefore, the value of $y_{\min }$ can range from 0.0006 for an electron with an energy of $40 \mathrm{GeV}$ to 0.0014 for a $100 \mathrm{GeV}$ electron. A systematic uncertainty from using a $y_{\min }$ value of 0.001 is determined by creating simulation templates of the $M_{T}$ distribution with $y_{\min }$ fixed at a value ranging between 0.0005 and 0.002 for each template. These templates are fitted to the $M_{T}$ distribution obtained with the simulation using the default 
value of $y_{\min }=0.001$ and a maximum shift in the fitted width of $8 \mathrm{MeV}$ is found.

The cross-section for a photon scattering off an electron (Compton scattering) becomes significant for low energy photons. The contribution to the total cross-section from Compton scattering increases from about $10 \%$ for a photon of energy $100 \mathrm{MeV}$ to around $60 \%$ for a $10 \mathrm{MeV}$ photon in silicon. The differential cross-section for Compton scattering can be written as

$$
\frac{d \sigma}{d y_{C}}=\frac{1}{y_{C}}+y_{C}
$$

where $y_{C}$ is the fraction of the incident photon energy carried by the scattered photon. A systematic on the $\mathrm{W}$ width resulting from neglecting Compton scattering is found by fitting simulation templates generated with and without Compton scattering and is found to be $7 \mathrm{MeV}$.

A summary of the systematic uncertainties on the $\mathrm{W}$ width from the simulation of energy loss by electrons is shown in Table 6.1.

\begin{tabular}{|l|cccc|}
\hline & iterations & bremsstrahlung & Compton scattering & total \\
\hline \hline Electron channel & $8 \mathrm{MeV}$ & $8 \mathrm{MeV}$ & $7 \mathrm{MeV}$ & $13 \mathrm{MeV}$ \\
\hline
\end{tabular}

Table 6.1: Systematic uncertainties on the $\mathrm{W}$ width in $\mathrm{W} \rightarrow e \nu$ events from the simulation of energy loss by electrons, where the individual uncertainties have been added in quadrature to obtain the total uncertainty.

\section{$6.2 \mathrm{COT}$}

A charged particle passing through the COT ionises the atoms of the gaseous mixture and its trajectory is bent by the magnetic field to leave a curved track allowing the transverse momentum and the charge of the particle to be determined. The event generator returns the true momenta of the leptons which must be smeared to account for detector resolution effects and scaled to account for inaccuracies in the determination of the magnetic field, COT alignment and the lepton energy loss model. 


\subsubsection{Momentum Scale and Resolution}

The curvature of a track $(\rho)$ is related to its transverse momentum in the following way

$$
\rho=\frac{q}{p_{T}}
$$

where $q$ is the charge of the particle. The resolution on this curvature can be defined as the difference between the generated curvature and the reconstructed curvature

$$
\Delta \rho=\left(\frac{q}{p_{T}}\right)_{\text {gen }}-\left(\frac{q}{p_{T}}\right)_{\text {recon }} .
$$

The $\Delta \rho$ distribution for lepton tracks is obtained from $\mathrm{W} \rightarrow \mu \nu$ CdfSim events for four categories of selection requirements on the number of axial and stereo superlayers traversed by the particle. These are:

- Four $N_{\text {seg }}^{\text {axial }}$ and four $N_{\text {seg }}^{\text {stereo }}$ layers.

- Four $N_{\text {seg }}^{\text {axial }}$ and three $N_{\text {seg }}^{\text {stereo layers. }}$

- Three $N_{\text {seg }}^{\text {axial }}$ and four $N_{\text {seg }}^{\text {stereo layers. }}$

- Three $N_{\text {seg }}^{\text {axial }}$ and three $N_{\text {seg }}^{\text {stereo }}$ layers.

These distributions are shown in Figure 6.3. Figure 6.3(a) shows obvious tails in the distribution that are not Gaussian. This effect is also present in the other distributions, though to a lesser extent.

For each lepton track in the simulation, the number of axial and stereo superlayers traversed by the particle trajectory is obtained by sampling from the $N_{\text {seg }}^{\text {axial }}$ and $N_{\text {seg }}^{\text {stereo }}$ distributions obtained from $\mathrm{Z} \rightarrow \mu^{+} \mu^{-}$data. Given this $N_{\text {seg }}^{\text {axial }}$ and $N_{\text {seg }}^{\text {stereo }}$, the relevant $\Delta \rho$ distribution from Figure 6.3 is sampled to obtain the smeared curvature of the track.

This curvature resolution is based on CdfSim and it may need to be scaled to match the data. The standard calibration sample of $\mathrm{Z} \rightarrow \mu^{+} \mu^{-}$events is used to calibrate the 


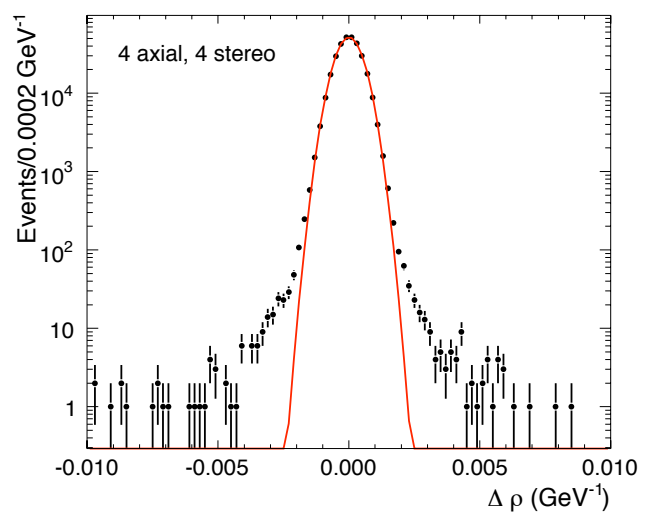

(a)

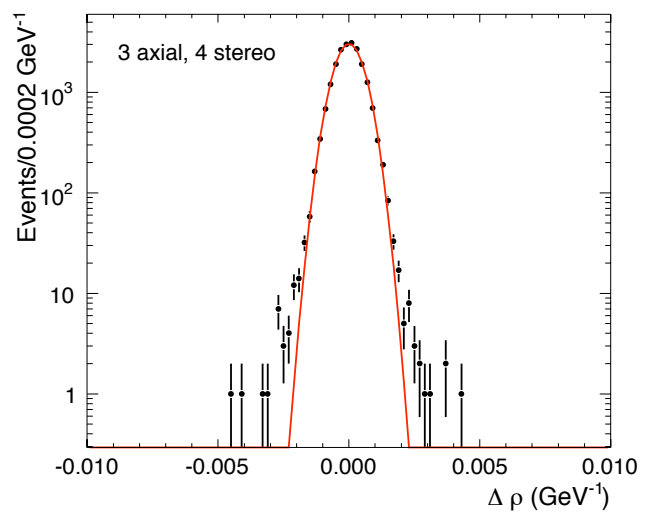

(c)

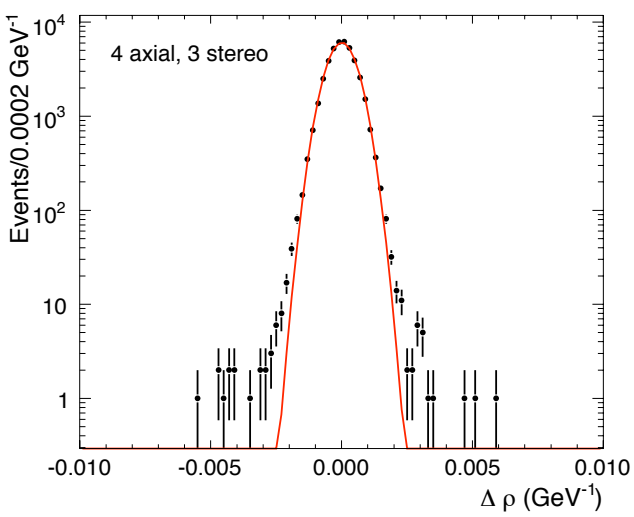

(b)

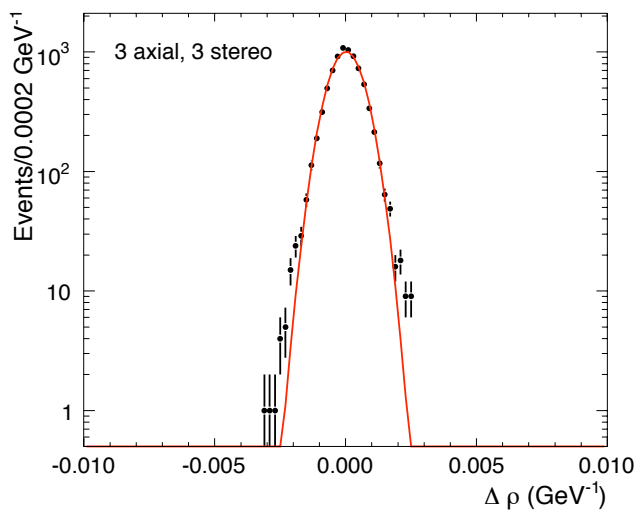

(d)

Figure 6.3: The $\Delta \rho$ distribution taken from $\mathrm{W} \rightarrow \mu \nu$ CdfSim events for muon tracks with (a) four $N_{\mathrm{seg}}^{\text {axial }}$ and four $N_{\text {seg }}^{\text {stereo }}$, (b) four $N_{\text {seg }}^{\text {axial }}$ and three $N_{\text {seg }}^{\text {stereo }}$, (c) three $N_{\text {seg }}^{\text {axial }}$ and four $N_{\text {seg }}^{\text {stereo }}$ and (d) three $N_{\text {seg }}^{\text {axial }}$ and three $N_{\text {seg }}^{\text {stereo }}$. The distributions are fitted with a Gaussian function, it is clear that the momentum resolution has non-Gaussian components.

COT scale and resolution. The invariant mass of the $\mathrm{Z}$ boson in the simulation is fitted to the data whilst varying a multiplicative factor $\mathrm{S}^{\text {res }}$ that scales $\Delta \rho$. The value of $\mathrm{S}^{\text {res }}$ that gives the best fit between the $\mathrm{Z}$ invariant mass distribution in data and simulation is found to be $\mathrm{S}^{\text {res }}=1.100 \pm 0.039$, i.e. the simulation needs an additional $10 \%$ smearing to match the data. The fit is shown in Figure 6.4.

The lepton track momenta are also scaled by a multiplicative factor $\mathrm{S}^{\mathrm{mom}}$ which is also found by fitting to the $\mathrm{Z}$ invariant mass distribution. The scale factor is found to be $\mathrm{S}^{\text {mom }}$ 
$=0.99891 \pm 0.00043$.

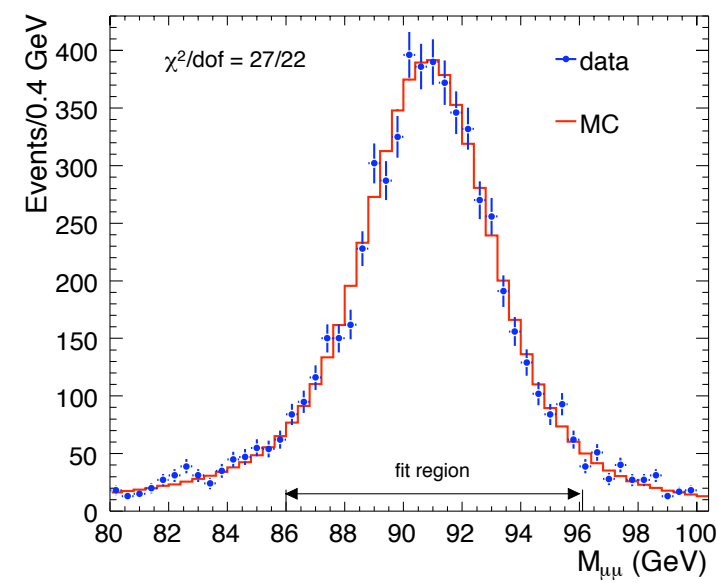

Figure 6.4: Fit to the $\mathrm{Z}$ invariant mass distribution in $\mathrm{Z} \rightarrow \mu^{+} \mu^{-}$candidate events to obtain the momentum scale and resolution.

Other track parameters such as $\phi$ and $\cot \theta$ are smeared with a Gaussian distribution with $\sigma_{\phi}=0.002$ and $\sigma_{\cot (\theta)}=0.011$ taken from CdfSim.

\subsubsection{Systematic Uncertainty}

The systematic uncertainty on the $\mathrm{W}$ width due to the momentum scale and resolution uncertainty is found by evaluating the shift in the width from varying $\mathrm{S}^{\mathrm{mom}}$ and $\mathrm{S}^{\text {res }}$ individually by $\pm 4 \sigma$ and $\pm 2 \sigma$ from the best fit values obtained from the $\mathrm{Z}$ invariant mass fit. The $1 \sigma$ uncertainty is then interpolated from this distribution.

It is assumed that CdfSim accurately predicts the shapes of the $\Delta \rho$ distributions. The validity of this assumption needs to be evaluated to investigate any possible bias in the momentum resolution. This can be done by altering the shapes of the $\Delta \rho$ distributions and fitting to the $E / p$ distribution in $\mathrm{W} \rightarrow e \nu$ events. It is observed from Figure 6.3 that the $\Delta \rho$ distributions can be split into a Gaussian component constituting the central region $|\Delta \rho|<$ 0.001 and a non-Gaussian component comprising of the tails of the distribution. The two components are sampled separately with a relative rate depending on their respective integrals. The non-Gaussian contribution can be varied by applying a multiplicative factor, 
$F^{\text {nGaus }}$ to the rate at which the non-Gaussian tails are sampled. The value of $F^{\text {nGaus }}$ can be obtained by fitting to $E / p$ distribution in the simulation whilst varying $F^{\mathrm{n} G a u s}$. Tighter cuts are made on the electron $E_{T}$ and $E_{\text {had }} / E_{\text {em }}$ variables to exclude events where there is considerable leakage of the electromagnetic shower into the hadronic calorimeter as this can affect the low $E / p$ region. The $E / p$ fit is performed in five bins to decouple the resolution on the track curvature from the calorimeter resolution and the following relationship between $\mathrm{S}^{\text {res }}$ and $F^{\text {nGaus }}$ is obtained

$$
\mathrm{S}^{\mathrm{res}}=1.14-0.04 \times F^{\mathrm{nGaus}}
$$

where

$$
F^{\text {nGaus }}=1.03 \pm 0.28_{\text {stat. }} \pm 0.34_{\kappa} \pm 0.01_{S_{\text {mat }}} \pm 0.08_{\text {background }}
$$

with contributions to the uncertainty on $F^{\text {nGaus }}$ from the statistical uncertainty of the fit, the calorimeter resolution $(\kappa)$ which is described in detail in Section 6.5.1, the uncertainty on the material scale $\left(S_{\text {mat }}\right)$ (described in the Section 6.3) and QCD background. The uncertainty on $\Gamma_{W}$ from $F^{\text {nGaus }}$ is found to be $16 \mathrm{MeV}$. Table 6.2 summarises the systematic uncertainties on the width from the momentum scale and resolution.

\begin{tabular}{|c|ccc|}
\hline & $\Delta \Gamma_{W}\left(\mathrm{~S}^{\text {mom }}\right)$ & $\Delta \Gamma_{W}\left(\mathrm{~S}^{\text {res }}\right)$ & $\Delta \Gamma_{W}\left(F^{\text {nGaus }}\right)$ \\
\hline \hline Muon channel & $17 \mathrm{MeV}$ & $21 \mathrm{MeV}$ & $16 \mathrm{MeV}$ \\
\hline
\end{tabular}

Table 6.2: Systematic uncertainties on the $\mathrm{W}$ width in $\mathrm{W} \rightarrow \mu \nu$ events from the COT momentum scale, resolution and the non-Gaussian fraction.

\subsection{Material Scale}

The amount of material in the silicon tracker simulation will affect the rate of bremsstrahlung. Any discrepancy in the overall amount of passive material in the simulation compared to the data is corrected using the $E / p$ distribution.

The $E / p$ distribution is sensitive to the rate of bremsstrahlung as bremsstrahlung photons are usually included in the transverse energy measurement in the calorimeter but 
not in the momentum measurement. This results in a distribution, shown in Figure 6.7 for $\mathrm{W} \rightarrow e \nu$ events, that is not symmetric about 1.0 but has a tail at high $E / p$ due to hard bremsstrahlung events. The tail of the $E / p$ distribution can be used to constrain the amount of material presented to electrons and photons by scaling the fractional radiation length obtained from SiliMap by a multiplicative factor, $S_{\text {mat }}$.

This scale factor is found by fitting to the $E / p$ distribution in $\mathrm{W} \rightarrow e \nu$ events in the region, $0.8<E / p<2.0$. The $E / p$ cut is not made for the fit and the electron $E_{T}$ cut is raised from $25 \mathrm{GeV}$ to $30 \mathrm{GeV}$ to give a QCD background of $(1.29 \pm 0.25) \%$. The material scale is found to be

$$
S_{\text {mat }}=1.033 \pm 0.007_{\text {stat. }} \pm 0.007_{\text {background }}
$$

with the statistical uncertainty and the uncertainty from QCD background. A material scale of 1.033 effectively means that the simulation based on the default SiliMap requires $3.3 \%$ more passive material to match the data.

\subsection{ToF and Solenoid}

As shown in Figure 6.1, after exiting the COT, electrons and photons enter the Timeof-Flight (ToF) detector and solenoid which present 0.1 and 0.8 of a radiation length respectively. There is therefore non-negligible energy loss by electrons and photons before they enter the calorimeter system. The CEM presents a total of 18 radiation lengths, and whereas this ensures that $\sim 98 \%$ of an electromagnetic shower is contained within the calorimeter, there can be some leakage out of the back of the calorimeter into the hadronic compartment (CHA). Both of these effects, energy loss in the ToF/solenoid system and leakage into the CHA, can reduce the amount of energy measured in the CEM and need to be accounted for in order to make an accurate measurement of the electron energy. 
These effects are simulated in the fast simulation by sampling from a distribution obtained from CdfSim which describes the variation of the fraction of energy deposited in the CEM as a function of the incident electron energy, shown in Figure 6.5(a) and the incident photon energy, shown in Figure 6.5(b). This takes into account any possible correlations between the energy loss in the ToF/solenoid and energy leakage into the CHA.

The distributions also show the relative contributions to the CEM energy from electrons, positrons and photons. At low electron energies (Figure 6.5(a)), the energy reaching the CEM is almost entirely from radiated photons as the incident electron is absorbed in the ToF and solenoid. At high energies, electrons and photons contribute almost equally to the energy deposited in the CEM as the total energy deposited approaches $98 \%$ of the incident electron energy. Comparing Figure 6.5(a) and Figure 6.5(b) shows that electrons lose more energy than photons owing to their ionisation of the material with no electron energy reaching the CEM at low energies whilst the fraction of the photon energy deposited in the CEM is always above $70 \%$.

\subsection{Calorimeter}

The most important measured quantity for electrons from $\mathrm{W}$ decay is the energy measured in the calorimeter. The calorimeter simulation takes the event generator electron energy corrected for all the energy loss effects described in the previous sections. The electron track is extrapolated to the position of a tower in the calorimeter. Photons from QED radiation and bremsstrahlung are also propagated to the calorimeter and if they end up in the same tower as the electron their energy is merged with the electron energy. If a photon ends up in one of the neighbouring towers in $\eta$, the tower is also merged with the electron tower provided that both towers are in the central calorimeter and do not extend over the crack separating the two halves of the detector. All the fiducial cuts requiring the electron to be in the central calorimeter and in an instrumented region of the detector as described 

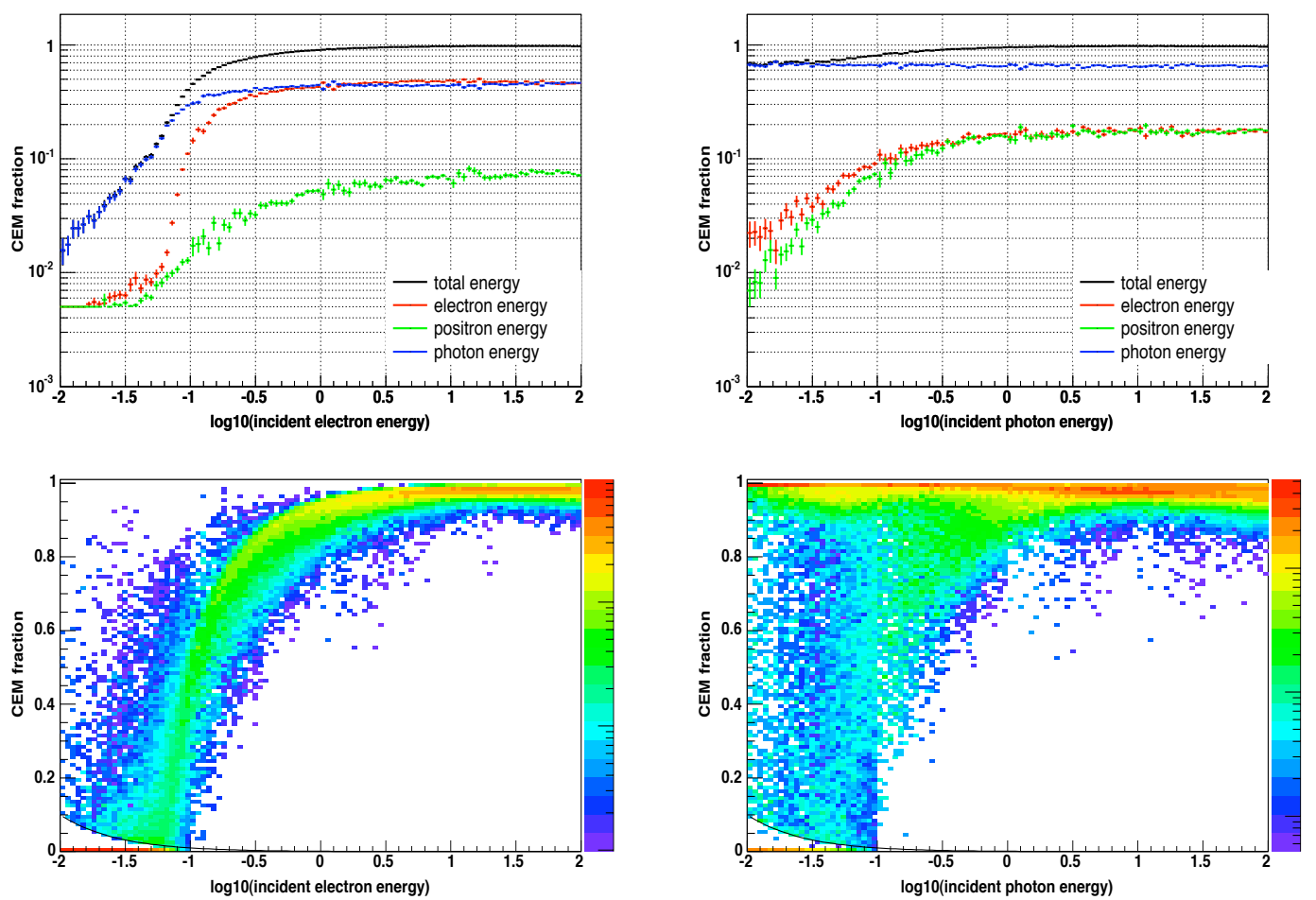

(a)

(b)

Figure 6.5: Top: The energy fraction deposited in the CEM as a function of the (a) incident electron energy (in $\mathrm{GeV}$ ) and (b) incident photon energy (in $\mathrm{GeV}$ ) and the way in which this energy is distributed between bremsstrahlung photons and conversion electrons and positrons. Bottom: The distribution of the energy fractions that are sampled in the fast simulation for every (a) electron and (b) photon exiting the COT.

in Chapter 4 are simulated. The electron energy is therefore a sum over the true energies of the electrons and photons (corrected for energy loss) that are propagated to the twotower cluster. This energy needs to be corrected for the response of the electromagnetic calorimeter and smeared so it matches the energy measured in the data.

\subsubsection{CEM Scale and Resolution}

The response of the CEM to electrons and photons is parameterised as a multiplicative scale factor. The resolution of the CEM is parameterised as

$$
\frac{\sigma_{E}}{E}=\frac{13.5 \%}{\sqrt{E_{\mathrm{T}}}} \oplus \kappa
$$


where the $13.5 \%$ stochastic term is obtained from test beam data and $\kappa$ is a constant term accounting for additional sources of resolution resulting from residual variations in the calorimeter response and calibration.

There are several detector effects causing variations in the calorimeter response. Light attenuation in the scintillator results in variations of the response as a function of position within a calorimeter tower. There is reduced response near the centre of the towers because light produced in this region has to travel over a longer distance to the wavelength shifters that carry the signals to the PMTs on either side of the towers, resulting in greater attenuation. The calorimeter response is also non uniform as a function of time and this is thought to be due to the ageing of the scintillators over time resulting in a reduced response. These effects are corrected for in offline calibrations which aim to flatten the calorimeter response.

Any residual effects causing non-uniformity are included in the constant term $\kappa$, where this term can be divided into two components, one from contributions to the resolution that are correlated between all electrons in the event $\left(\kappa^{\text {corr }}\right)$ and one from contributions that are uncorrelated between electrons $\left(\kappa^{\text {uncorr }}\right)$. In general there are two techniques that can be employed to calibrate the calorimeter energy scale and resolution; measurement of the invariant mass of the $\mathrm{Z}$ boson in $\mathrm{Z} \rightarrow e^{+} e^{-}$events and measurement of the $E / p$ ratio in the vicinity of the peak. Figure 6.6 shows the mean $E / p$ of electrons in $\mathrm{W} \rightarrow e \nu$ events as a function of time. It shows that there is a residual dependence on time after all the offline corrections have been applied. The correlated contribution $\kappa^{\text {corr }}$ is taken from the root-mean-square of this distribution, giving $\kappa^{\mathrm{corr}}=0.29 \%$.

The uncorrelated contribution $\kappa^{\text {uncorr }}$ and the scale $S^{\mathrm{CEM}}$ is obtained by independent fits to the $E / p$ distribution in $\mathrm{W} \rightarrow e \nu$ events and the invariant mass in $\mathrm{Z} \rightarrow e^{+} e^{-}$events. Simulation templates of the $E / p$ distribution using different input values of $\kappa^{\text {uncorr }}$ and $S^{\mathrm{CEM}}$ are used to fit to the peak of the $E / p$ distribution $(0.9<E / p<1.1)$ in $\mathrm{W} \rightarrow e \nu$ 


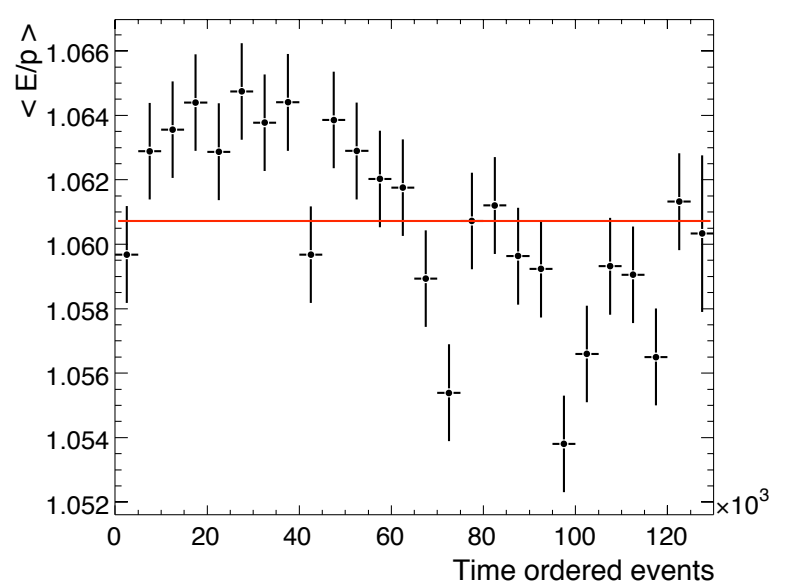

Figure 6.6: The variation of the $\langle E / p\rangle$ as a function of time in $\mathrm{W} \rightarrow e \nu$ events.

data. The fit is shown in Figure 6.7 and the values obtained are

$$
\begin{array}{r}
\kappa^{\text {uncorr }}=\left(0.947 \pm 0.049_{\text {stat. }} \pm 0.147_{\text {track }} \pm 0.056 S_{\text {mat }}\right) \% \\
S^{\mathrm{CEM}}=1.02356 \pm 0.00021_{\text {stat. }} \pm 0.00044_{\text {track }} \pm 0.00017_{S_{\text {mat }}}
\end{array}
$$

where there are contributions to the uncertainty from the track momentum scale and resolution and the material scale factor. A fit to the $E / p$ distribution in the narrower range $0.96<E / p<1.1$ yields $\kappa^{\text {uncorr }}=\left(0.64 \pm 0.11_{\text {stat. }}\right) \%$.

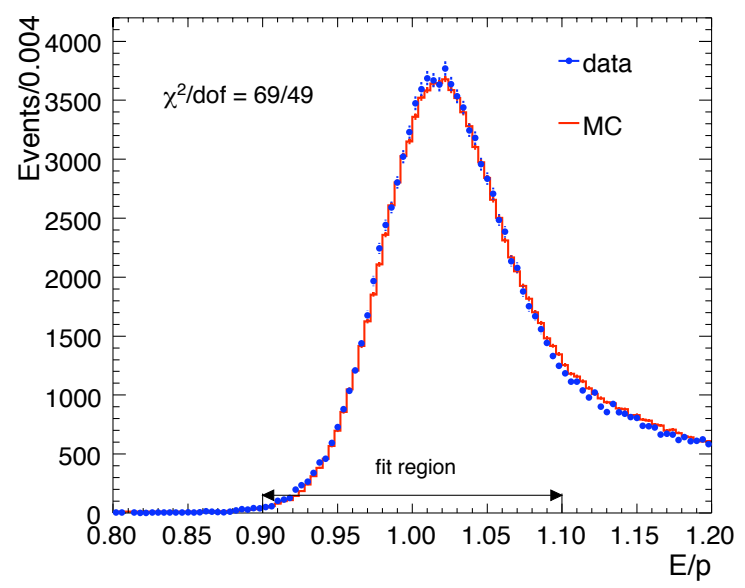

Figure 6.7: A fit to the $E / p$ distribution in $\mathrm{W} \rightarrow e \nu$ events to determine the calorimeter scale and the uncorrelated contribution to the detector resolution. 
Similarly, templates of the $M_{e e}$ distribution with varying input values for $\kappa^{\text {uncorr }}$ and $S^{\mathrm{CEM}}$ are fitted to the $M_{e e}$ peak in $\mathrm{Z} \rightarrow e^{+} e^{-}$data in the range $86<M_{e e}<96 \mathrm{GeV}$. The fit is shown in Figure 6.8 and the values obtained are

$$
\begin{array}{r}
\kappa^{\text {uncorr }}=(1.49 \pm 0.29) \% \\
S^{\text {CEM }}=1.02439 \pm 0.00078 .
\end{array}
$$

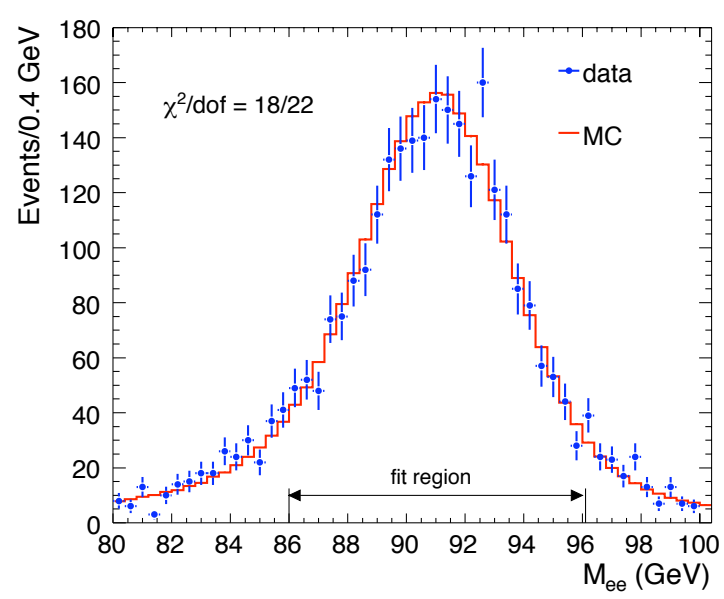

Figure 6.8: A fit to the $M_{e e}$ distribution in $\mathrm{Z} \rightarrow e^{+} e^{-}$events to determine $\kappa^{\text {uncorr }}$ and $S^{\mathrm{CEM}}$.

The values of $S^{\mathrm{CEM}}$ from the two fits are consistent with each other and are combined in a weighted average to give

$$
S^{\mathrm{CEM}}=1.02382 \pm 0.00043
$$

The values of $\kappa^{\text {uncorr }}$ obtained from the $M_{e e}$ fit and the $E / p$ fit in both fit ranges are combined with a weighted average and the uncertainty is conservatively chosen to cover all three $\kappa^{\text {uncorr }}$ values obtained from the above fits

$$
\kappa^{\text {uncorr }}=1.08_{-0.44}^{+0.41 \%} \text {. }
$$




\section{Systematic uncertainty}

The uncertainty on $\Gamma_{W}$ due to the uncertainty on the calorimeter scale and resolution is found by varying the parameters by $\pm 2 \sigma$ and $\pm 4 \sigma$ and interpolating the $1 \sigma$ uncertainty from this. The uncertainty on $\Gamma_{W}$ obtained from $S^{\mathrm{CEM}}$ and $\kappa$ is shown in Table 6.3.

\begin{tabular}{|c|cc|}
\hline & $\Delta \Gamma_{W}\left(S^{\mathrm{CEM}}\right)$ & $\Delta \Gamma_{W}(\kappa)$ \\
\hline \hline Electron channel & $17 \mathrm{MeV}$ & $31 \mathrm{MeV}$ \\
\hline
\end{tabular}

Table 6.3: Systematic uncertainties on the $\mathrm{W}$ width in $\mathrm{W} \rightarrow e \nu$ events from the uncertainty on the calorimeter scale and resolution.

\subsubsection{Muon Energy Simulation}

Muons leave a small fraction of their energy in the calorimeter via ionisation. Whereas the energy deposited by a muon is small and not used in the transverse mass calculation, an accurate simulation of the muon energy is required to simulate the $E_{\text {em }}$ cut which is used to identify muons in the data and also for the simulation of the recoil. The energy deposited by muons in the calorimeter is simulated by sampling from the energy distribution obtained from a sample of cosmic ray muons. The energy of QED photons that end up in the same tower as the muon is added to the muon energy.

\subsubsection{Underlying Energy Simulation}

In addition to the electrons and photons from the electromagnetic shower that enter the electron energy cluster in the data, there is overlapping energy from particles unassociated with the interaction producing the $\mathrm{W}$ boson. This energy is referred to as the underlying energy and has contributions from the low $p_{T}$ remnants of the $p \bar{p}$ collision and from additional interactions between a proton and antiproton within the same bunch crossing.

This energy is simulated by sampling from an energy distribution obtained from $\mathrm{W} \rightarrow e \nu$ events in data. The energy in a two-tower cluster in the calorimeter rotated away from the electron cluster in $\phi$ but with the same $\eta$ as the electron is studied as a 
function of the total $\Sigma E_{\mathrm{T}}$ of the event. The energy has a linear dependence on the $\Sigma E_{\mathrm{T}}$ since as the instantaneous luminosity and hence the $\Sigma E_{\mathrm{T}}$ increases, there is more energy flow in the calorimeter so the average energy deposited in a two-tower cluster is larger. This effect is simulated by sampling from the two-tower distribution and then scaling this energy according to the linear dependence on $\Sigma E_{\mathrm{T}}$, such that there is more overlapping energy for an event with large $\Sigma E_{\mathrm{T}}$. The simulation of the $\Sigma E_{\mathrm{T}}$ distribution will be described in detail in Chapter 7.

The underlying energy is simulated for both electrons and muons. In the data, the overlapping underlying energy makes it more likely for a muon to fail the $E_{\mathrm{em}}$ cut and this effect is incorporated in the simulation by adding a $\Sigma E_{\mathrm{T}}$ dependent underlying energy to the energy of a muon.

\subsubsection{Calorimeter Non-linearity}

The response of the calorimeter to particles with different energies may not be uniform and this non-linearity in the response can arise from several potential sources. The effects giving rise to an effective non-linearity such as leakage into the CHA, energy loss in the ToF/solenoid have already been discussed. However, there is a residual non-linearity resulting in a different response for high and low energy deposits. This is thought to be due to the variation of the calorimeter response as a function of calorimeter depth. This is described in more detail in [61], together with a description of how it is simulated and how the systematic on the width is evaluated. The systematic uncertainty on $\Gamma_{W}$ from the calorimeter non-linearity is found to be $12 \mathrm{MeV}$.

\subsection{Acceptances and Efficiencies}

The fiducial requirements on electrons and muons from $\mathrm{W}$ decay affect their kinematic distributions and hence the transverse mass. The fiduciality requirements and the efficiency of the electron and muon selection requirements need to be modelled in the simulation. 
This is described in detail in [61]. The electron and muon trigger efficiencies are high and well modelled and also described in detail in [61]. The systematic uncertainty on the $\mathrm{W}$ width from the acceptances and efficiencies is $3 \mathrm{MeV}(4 \mathrm{MeV})$ and $10 \mathrm{MeV}(6 \mathrm{MeV})$ respectively for the electron (muon) channel.

\subsection{Summary}

A summary of the systematic uncertainties associated with the simulation of leptons is shown in Table 6.4 for the electron and muon channel. The uncertainties contributing to the simulation of electrons arise from the uncertainties on the calorimeter scale and resolution, the non-linear response of the calorimeter to electrons with different energies, the modelling of the energy loss and the detector acceptance and electron selection efficiencies. The uncertainties contributing to the muon simulation are dominated by the uncertainty on the tracker scale and resolution and the shape of the resolution distribution.

\begin{tabular}{|l|cc|}
\hline & $\Delta \Gamma_{W}(\mathrm{~W} \rightarrow e \nu)$ & $\Delta \Gamma_{W}(\mathrm{~W} \rightarrow \mu \nu)$ \\
\hline \hline energy loss simulation & $13 \mathrm{MeV}$ & - \\
silicon material scale & $2 \mathrm{MeV}$ & - \\
COT scale & - & $17 \mathrm{MeV}$ \\
COT resolution & - & $21 \mathrm{MeV}$ \\
COT resolution shape & - & $16 \mathrm{MeV}$ \\
calorimeter scale & $17 \mathrm{MeV}$ & - \\
calorimeter resolution & $13 \mathrm{MeV}$ & - \\
calorimeter non-linearity & $12 \mathrm{MeV}$ & - \\
acceptances & $3 \mathrm{MeV}$ & $4 \mathrm{MeV}$ \\
efficiencies & $10 \mathrm{MeV}$ & $6 \mathrm{MeV}$ \\
\hline
\end{tabular}

Table 6.4: Summary of the systematic uncertainties associated with the simulation of leptons. 


\section{Chapter 7}

\section{Recoil Reconstruction and Simulation}

The calculation of the transverse mass in $\mathrm{W} \rightarrow l \nu$ decays depends on the transverse energies of the charged lepton and the neutrino. The neutrino does not interact with the material of the detector and is not reconstructed. Its transverse energy is inferred from the missing transverse energy in the event which can be calculated using

$$
\overrightarrow{E_{T}^{v}} \equiv \overrightarrow{E_{T}}=\left(\overrightarrow{E_{T}}+\vec{U}\right)
$$

where $\overrightarrow{E_{T}}$ is the charged lepton transverse energy vector and $\vec{U}$ is the recoil vector. An accurate simulation of the recoil is important to accurately determine $E_{T}$ and hence the W transverse mass.

In addition, substituting the above equation into the transverse mass equation (Equation 3.10), a relationship to first order in $|\vec{U}| / E_{T}$ between the transverse mass and the component of $\vec{U}$ in the direction parallel to the charged lepton $\left(U_{\|}\right)$can be obtained in the limit $|\vec{U}| \ll E_{T}$. The transverse mass becomes

$$
M_{T} \approx 2\left|\overrightarrow{E_{T}}\right|+U_{\|}
$$

The magnitude of the recoil in the direction of the charged lepton is therefore strongly correlated with $M_{T}$; any bias in $U_{\|}$introduces a bias in the $M_{T}$ fit. It is therefore particularly important to simulate accurately. 
The recoil is defined as a sum over all the transverse energy in the calorimeter excluding that associated with the charged lepton. The energy in the calorimeter receives contributions from three major sources :

\section{Hard QCD sub-process}

The interacting partons from the incoming proton and antiproton emit gluon(s). $\mathrm{W}$ bosons produced in the interaction recoil against this gluon emission, acquiring a transverse momentum that is equal and opposite to it. The recoiling gluons hadronise and this jet-like recoil is reconstructed in the calorimeter with an energy and direction strongly correlated with the magnitude and direction of the boson $p_{\mathrm{T}}$.

\section{Soft underlying event}

This includes all the underlying low $p_{\mathrm{T}}$ interactions that are not correlated with the kinematics of the parton-parton interaction producing the $\mathrm{W}$ boson. Remnants of the $p \bar{p}$ collision, interactions between spectator quarks and additional interactions between a proton and antiproton within the same bunch crossing (multiple interactions) are the major processes that contribute to the underlying event energy. In general, the mean number of multiple interactions is linearly dependent on the instantaneous luminosity in the event. As the instantaneous luminosity increases, the average number of multiple interactions increase resulting in a larger flow of energy in the event.

\section{Bremsstrahlung photons}

Bremsstrahlung photons that are emitted collinear with the charged lepton often end up in the same or neighbouring towers and are merged with the lepton cluster. However, photons emitted at a wide angle to the lepton may deposit their energy in a tower that is included in the recoil calculation (described in Section 7.1.1). The energy from such photons will thus be added to the recoil sum. 
In general, the recoil is a conglomerate of particles from the above described contributions with a wide energy spectrum, making it a complex entity. On average, only around $70 \%$ of the hard QCD contribution is measured in the calorimeter owing to a number of factors. Some very low $p_{\mathrm{T}}$ particles are carried away by the magnetic field and never reach the calorimeter system whilst others are lost due to the incomplete angular coverage of the calorimeters. The recoil energy is also distributed between the electromagnetic and hadronic calorimeters with a significant amount of energy being deposited in the electromagnetic calorimeter from the decay of soft neutral pions into a pair of photons.

\subsection{Recoil reconstruction}

The recoil in the data is calculated as a vector sum over the energy in all the towers in the electromagnetic and hadronic sections of the central and plug calorimeters, excluding those associated with the lepton (the so-called 'knockout region', described in Section 7.1.1). The following formula is used in the calculation

$$
\vec{U}=\left(u_{x}, u_{y}\right)=\sum_{\text {towers }} E \sin \theta(\cos \phi, \sin \phi)
$$

where $\phi$ is the azimuthal angle of each calorimeter tower with respect to the position of the proton antiproton collision point in the detector and $\theta$ is the polar angle each tower makes with the $z_{0}$ position of the charged lepton track in the event, where in $\mathrm{Z} \rightarrow l^{+} l^{-}$ events, the average $z_{0}$ position of the two lepton tracks is used.

There are differences in the response of the central and plug calorimeters and the recoil energies obtained from the central and plug calorimeters are therefore scaled relative to each other to achieve a uniform response for the calorimeters. The central calorimeter tower energies are scaled up by $5 \%$ and the plug tower energies are scaled down by $7 \%$ [22].

In addition, a tower threshold of $100 \mathrm{MeV}$ is also applied to the electromagnetic and hadronic calorimeter towers in the $\mathrm{W}$ width analysis such that only towers with energy 
above $100 \mathrm{MeV}$ are included in the recoil calculation and the towers in the miniplug calorimeters, which cover the pseudorapidity region $3.6<|\eta|<5.1$, are also excluded. The $100 \mathrm{MeV}$ threshold is applied to reduce contribution from detector noise.

In the $\mathrm{W}$ mass analysis, towers in the region $|\eta|>2.6$ are excluded from the recoil calculation if they have $E_{T}<5 \mathrm{GeV}$. This is done to suppress the variation of the acceptance of the calorimeter as a function of $\phi$ which occurs as a result of the centre of the beam being offset from the detector centre (explained in detail in Section 7.1.2). This effect is enhanced in the forward regions of the detector where the towers are closest to the beam line and excluding towers in this region suppresses this variation in acceptance. The $5 \mathrm{GeV}$ cut ensures that jets from QCD events are not rejected.

\subsubsection{Knockout Region}

The recoil calculation sums over the transverse energies of all the towers in the calorimeter excluding those containing energy that is associated with the lepton. The region of the calorimeter that is not included in the recoil sum is referred to in the analysis as the 'knockout region'. Whereas most of the shower energy of an electron from a $\mathrm{W} \rightarrow e \nu$ decay is deposited in two calorimeter towers, there can be some leakage into a neighbouring tower particularly if the electron showers close to the tower edge. In addition, photons from bremsstrahlung can also deposit their energy in one of the neighbouring towers.

A nine tower region around the electron tower with the average energy in each tower is shown in Figure 7.1. The towers neighbouring the central electron tower are plotted such that the ones closest in distance to the position of the electron shower are those at positive $\Delta \phi$ and positive $\Delta \eta$. The average energy per tower in the electromagnetic and hadronic calorimeter is $33 \mathrm{MeV}$ and $10 \mathrm{MeV}$ respectively. Towers with energy significantly larger than this average are assumed to contain contributions from leakage and bremsstrahlung and form the knockout region. The knockout region for the electron is shown by the 7-tower shaded region in Figure 7.1. 
The muon is a minimum ionising particle and does not shower in the calorimeter. The muon therefore has a smaller knockout region comprising of 3 towers, the muon tower and the two neighbouring $\eta$ towers as shown in Figure 7.2.

In $\mathrm{Z} \rightarrow e^{+} e^{-}$events, 7 towers are knocked out for each electron resulting in 14 towers in total being excluded from the recoil sum and in $\mathrm{Z} \rightarrow \mu^{+} \mu^{-}$events 6 towers in total are excluded.
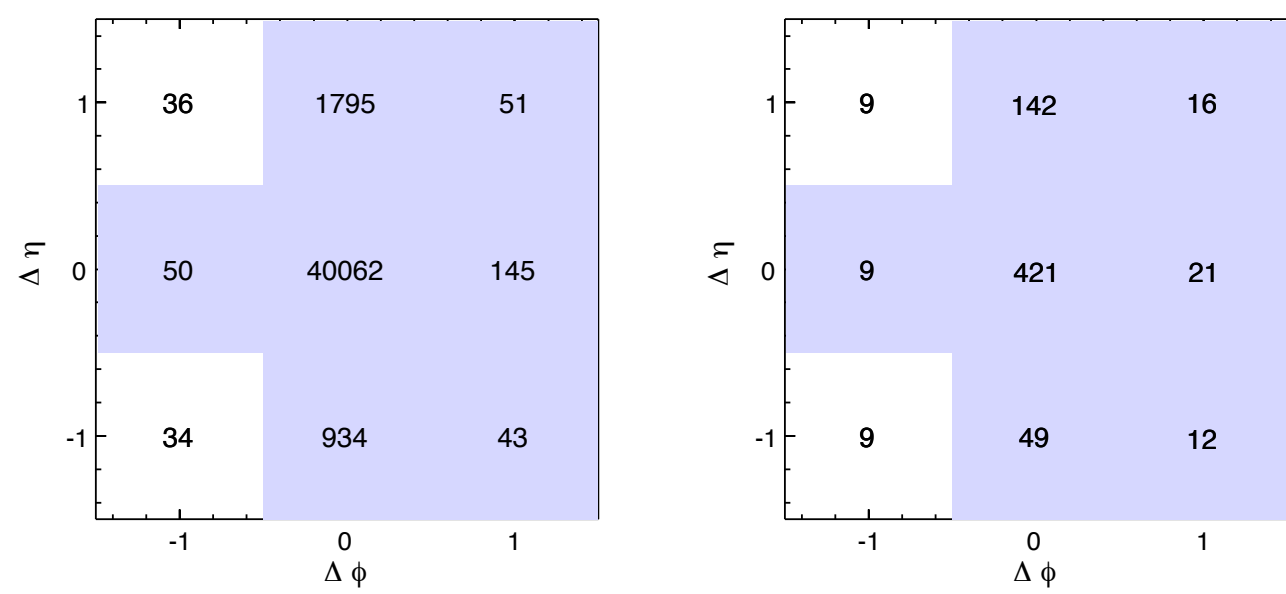

Figure 7.1: The energy (in $\mathrm{MeV}$ ) in the $3 \times 3$ tower region around the electron tower at $(\Delta \phi, \Delta \eta)=(0,0)$ for the electromagnetic calorimeter (left) and hadronic calorimeter (right) in the $\mathrm{W}$ width analysis. The shaded towers comprise the knockout region and are not included in the recoil calculation.

For the $\mathrm{W}$ mass analysis, the neighbouring towers in a 3 by 3 region around the lepton tower is shown for the electron in $\mathrm{W} \rightarrow e \nu$ events and the muon in $\mathrm{W} \rightarrow \mu \nu$ events in Figures 7.3 and 7.4 respectively. The average instantaneous luminosity for the $2.4 \mathrm{fb}^{-1}$ dataset used for the $\mathrm{W}$ mass analysis is more than twice as high as the average instantaneous luminosity in the dataset used for the $\mathrm{W}$ width analysis, as shown in Figure 7.13. This results in a greater number of multiple interactions per event and more energy flow into the calorimeter. The average energy in an electromagnetic and hadronic tower for the $\mathrm{W}$ mass analysis is $51 \mathrm{MeV}$ and $15 \mathrm{MeV}$ respectively. The number of towers knocked out for an electron and muon are the same as those for the $\mathrm{W}$ width analysis. 

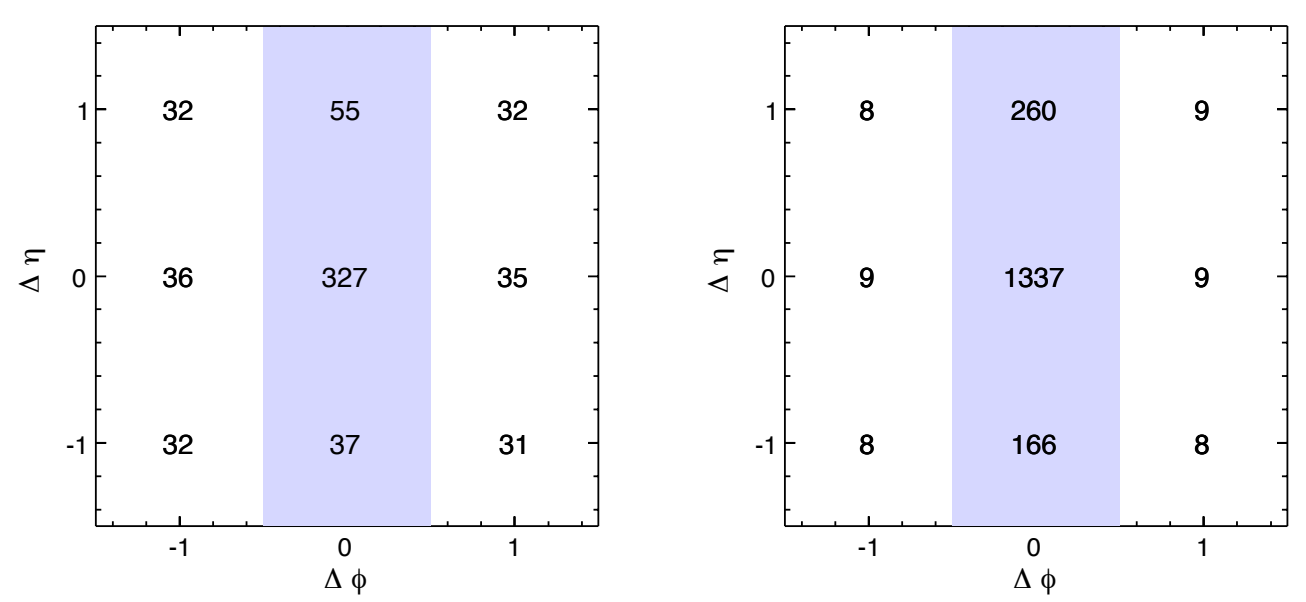

Figure 7.2: The $3 \times 3$ tower region around the muon tower at $(\Delta \phi, \Delta \eta)=(0,0)$ for the electromagnetic calorimeter (left) and hadronic calorimeter (right) in the $\mathrm{W}$ width analysis. The shaded towers comprise the knockout region and are not included in the recoil calculation.

The removal of the knockout region from the recoil not only removes the energy associated with the lepton but also overlapping recoil energy. The average recoil energy being excluded from the recoil sum in the $\mathrm{W}$ width and mass analyses is obtained from studying a region of the detector that is separated by $\Delta \phi= \pm 90^{\circ}$ from the lepton, as explained in detail in Section 7.2.1, and is found to be

$$
\begin{gathered}
E_{\mathrm{em}}=233 \pm 1 \mathrm{MeV}, E_{\text {had }}=68 \pm 1 \mathrm{MeV}, E_{\text {tot }}=301 \pm 2 \mathrm{MeV}(\text { W Width }) \\
E_{\mathrm{em}}=357 \pm 0.5 \mathrm{MeV}, E_{\text {had }}=105 \pm 0.3 \mathrm{MeV}, E_{\text {tot }}=462 \pm 0.7 \mathrm{MeV} \text { (W Mass) }
\end{gathered}
$$

where only the statistical errors are shown. This underlying energy lies in the direction of the lepton and can shift $U_{\|}$. It therefore needs to be carefully accounted for and it will be explained later in the chapter how the removal of this overlapping recoil energy is simulated.

\subsection{2 $\phi_{U}$ Modulation Correction}

The recoil vector is expected to be azimuthally symmetric with no preferred $\phi$ direction. However, the acceptance of the calorimeter has a dependence on the position of the beam 

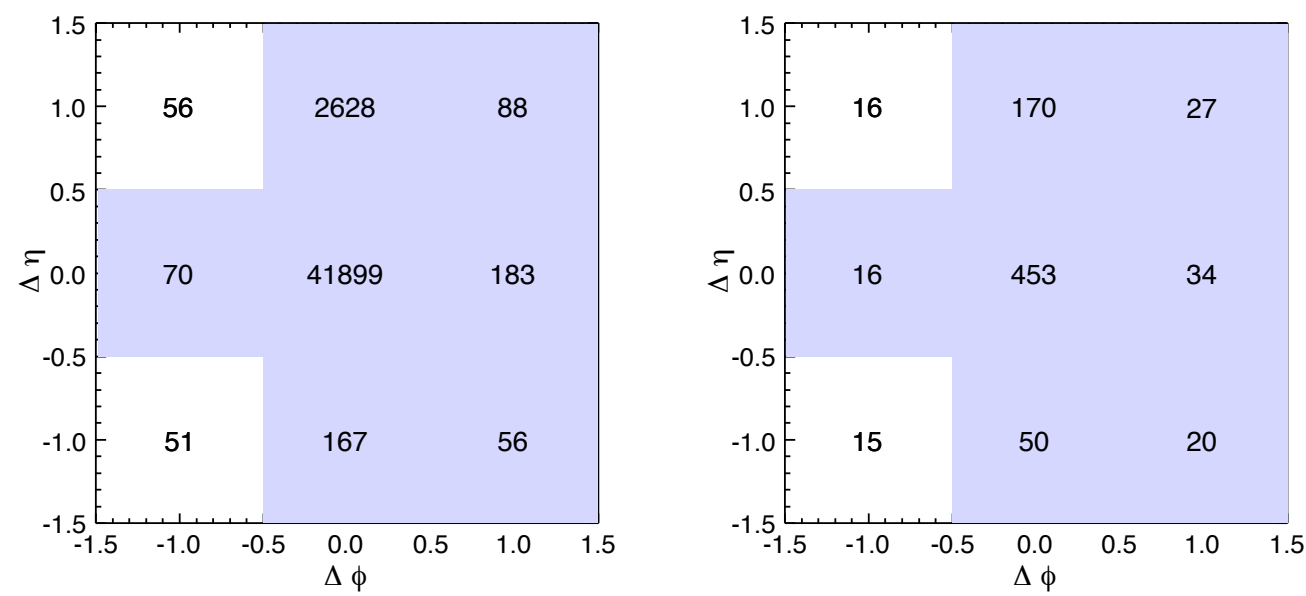

Figure 7.3: The energy (in $\mathrm{MeV}$ ) in the $3 \times 3$ tower region around the electron tower at $(\Delta \phi, \Delta \eta)=(0,0)$ for the electromagnetic calorimeter (left) and hadronic calorimeter (right) in the W mass analysis. The shaded towers comprise the knockout region and are not included in the recoil calculation.
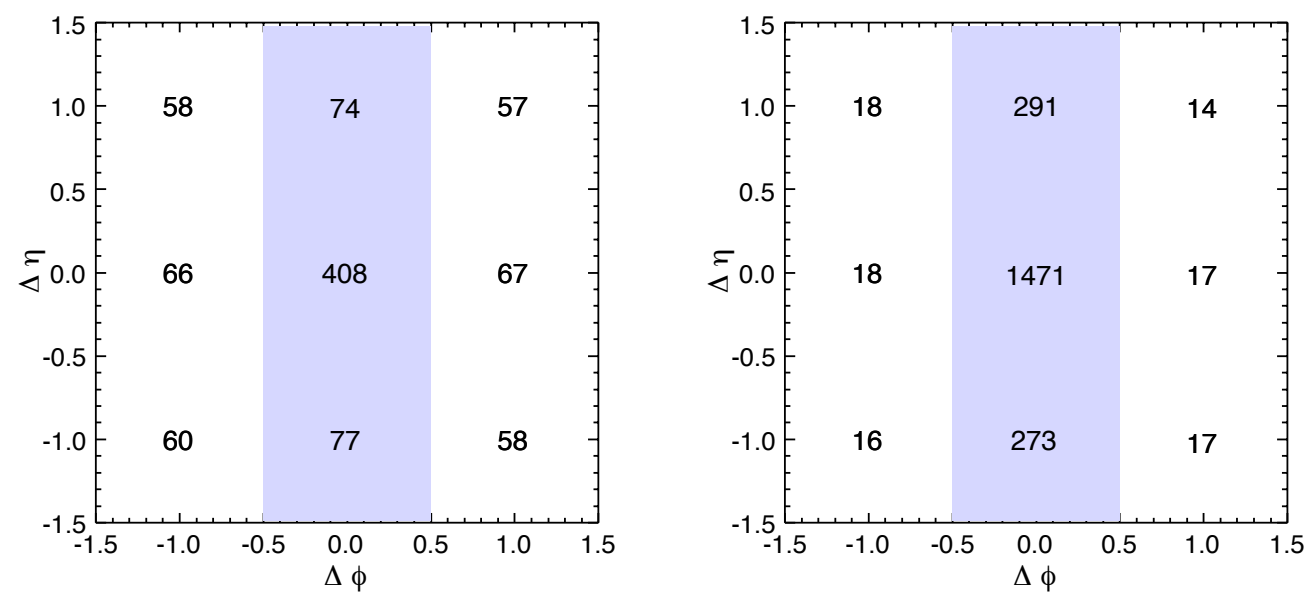

Figure 7.4: The $3 \times 3$ tower region around the electron tower at $(\Delta \phi, \Delta \eta)=(0,0)$ for the electromagnetic calorimeter (left) and hadronic calorimeter (right) in the $\mathrm{W}$ mass analysis. The shaded towers comprise the knockout region and are not included in the recoil calculation.

line with respect to the detector position. The centre of the beam is offset from the detector centre and this results in some towers of the calorimeter being closer to the interaction point and having a larger acceptance and thus a larger average measured energy. This produces a variation of the calorimeter response as a function of $\phi$.

In the $\mathrm{W}$ width analysis, a $17 \%$ modulation in the $\phi$ distribution of the recoil $\left(\phi_{U}\right)$ was 
observed, some of which was corrected by accounting for the beam offset and calculating the $E_{T}$ in the calorimeter towers with respect to the beam position in the detector and not the origin $(0,0)$. A remaining $11 \%$ modulation was observed and this is shown in Figure 7.5.

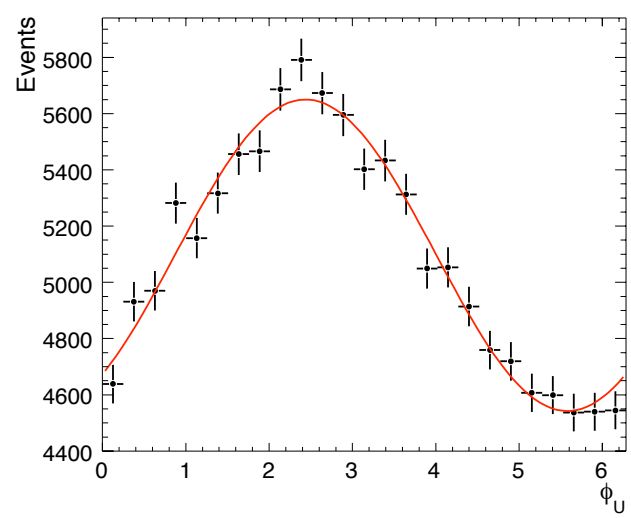

Figure 7.5: The modulation in $\phi_{U}$ after correcting for the beam offset.

In the $\mathrm{W}$ mass analysis, it is noted that any residual modulation in $\phi_{U}$ after accounting for the beam offset can be corrected by artificially shifting the position of the plug calorimeter with respect to the tracker system by an unphysical amount. The corrections are obtained by studying the slopes of the mean of $U_{x, y}$ vs. luminosity as a function of detector $\eta$ in minimum-bias events. The shifts are shown in Table 7.1 for the east (tower number $<16$ ) and west (tower number $>35$ ) halves of the plug calorimeter and for three run ranges ${ }^{1}$, where the run ranges correspond to the detector shutdowns. The modulation in $\phi_{U}$ before and after applying these corrections is shown in Figure 7.6 for $\mathrm{W} \rightarrow e \nu$ events. The distributions are fit using the function $y=a_{0} \times\left(1+a_{1} \times \sin \left(x+a_{2}\right)\right)$, where $a_{1}$ gives the fractional modulation and is measured to be $8 \%$ before the corrections and $0.7 \%$ after the corrections.

\footnotetext{
${ }^{1} \mathrm{~A}$ run is a period of continuous operation of data taking by the CDF detector in which a group of events are recorded to tape. Each run is given a number which is incremented with time.
} 


\begin{tabular}{|l|cc|}
\hline Run Period & $(\Delta x, \Delta y)$ (East) & $(\Delta x, \Delta y)$ (West) \\
\hline \hline Run $<168889$ & $-12 \mathrm{~mm},+15 \mathrm{~mm}$ & $-9 \mathrm{~mm},+24 \mathrm{~mm}$ \\
$168889<$ Run $<212133$ & $-9 \mathrm{~mm},+7 \mathrm{~mm}$ & $-9 \mathrm{~mm},+16 \mathrm{~mm}$ \\
Run $>212133$ & $-11 \mathrm{~mm},+5 \mathrm{~mm}$ & $-9 \mathrm{~mm},+10 \mathrm{~mm}$ \\
\hline
\end{tabular}

Table 7.1: The shifts in $(x, y)$ for the East and West halves of the plug calorimeter for three run ranges.

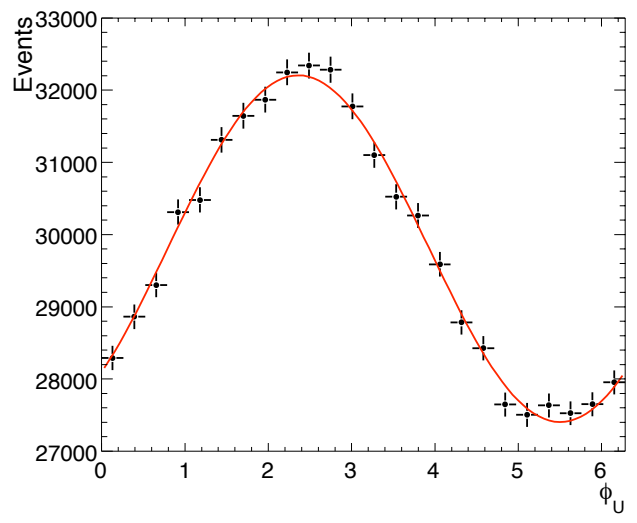

(a)

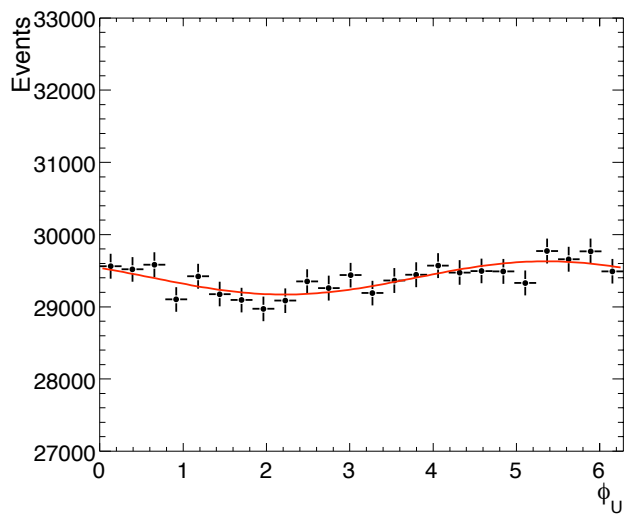

(b)

Figure 7.6: The modulation in $\phi_{U}$ in $\mathrm{W} \rightarrow e \nu$ events (a) before and (b) after the plug alignment corrections described in the text.

\subsection{Recoil Simulation}

The recoil is a complex system of particles from a combination of processes that are difficult to simulate on a particle-by-particle level. The simulation of the recoil takes the approach of an ad-hoc parameterisation where the parameters are obtained by fitting an aggregate model of the recoil to data. The three categories of processes contributing to the recoil energy are modelled and the total recoil can be written in terms of these as

$$
U=U_{\mathrm{QCD}}+U_{\mathrm{UE}}+U_{\mathrm{BREM}}
$$

The contribution from wide-angle QED and bremsstrahlung photons $\left(U_{\mathrm{BREM}}\right)$ is simulated using the fast simulation. The other two contributions, from initial state QCD radiation $\left(U_{\mathrm{QCD}}\right)$ and underlying energy $\left(U_{\mathrm{UE}}\right)$ are constrained by fits to $\mathrm{Z} \rightarrow l^{+} l^{-}$and minimumbias data. As in other parts of the analysis, $\mathrm{Z} \rightarrow l^{+} l^{-}$events provide a suitable sample to 
constrain the recoil since both leptons are reconstructed and the transverse momentum of the $\mathrm{Z}$ boson is well measured. In addition, the removal of the lepton energy, bremsstrahlung and overlapping underlying energy in the knockout region is also simulated.

The bremsstrahlung contribution to the recoil is simulated by adding to the recoil vector the energy of all QED photons in the simulation that are propagated to a calorimeter tower outside of the 7 (electrons) or 3 (muons) tower knockout region.

\subsubsection{Knockout Region Simulation}

The recoil energy calculated in the data is taken from the calorimeter with a 'hole' where the towers in the knockout region have been removed. Since the knockout region lies at the position of the lepton and the recoil projected in this direction can produce a significant bias in the $\mathrm{W}$ transverse mass, the knockout is an important effect to simulate.

The same number of towers as were removed from the data are also removed from the simulation for electrons and muons. Lateral leakage of the lepton energy is not simulated in the fast simulation used for the recoil so these towers contain only the lepton cluster energy (for muons this is the simulated $E_{\text {em }}$ energy) and photon energy from bremsstrahlung/conversions. The electromagnetic energy in the region around the lepton tower is shown in Figure 7.7 for the $\mathrm{W}$ mass analysis. The shaded region shows towers that are not included in the recoil simulation to emulate the knockout region in the data. The energy in towers outside of this region is added to the $U_{\mathrm{BREM}}$ recoil contribution.

In addition to energy from the lepton and bremsstrahlung, the knockout region in the data also removes overlapping recoil energy. In general, there are two ways of correcting for the 'hole' in the recoil left by the removal of this overlapping energy. This energy can be estimated and either added back to the data to recover the recoil energy removed from the knockout or subtracted from the simulation to simulate the 'hole' in the calorimeter present in the data. 

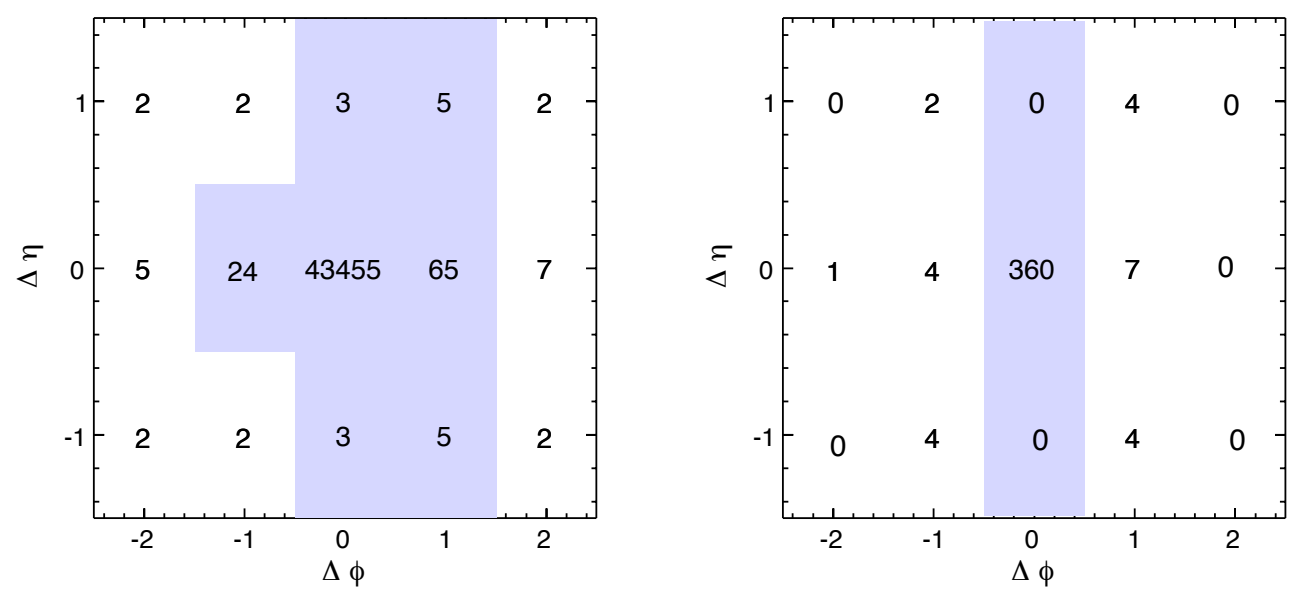

Figure 7.7: The average electromagnetic energy in towers around the electron (left) and muon (right) tower in $\mathrm{W} \rightarrow e \nu$ and $\mathrm{W} \rightarrow \mu \nu$ simulation events. The energy in the shaded region is excluded from the recoil calculation to simulate the knockout region in the data.

In the $\mathrm{W}$ width analysis, this correction is made in the data, whereas in the $\mathrm{W}$ mass analysis a 'hole' is created in the simulation by subtracting this energy from the recoil. The rest of this section will assume that the energy is being subtracted from the simulation.

Whereas the average energy to be subtracted is given in Equations 7.3 and 7.4, on an event by event basis there may be more or less energy depending on the following variables:

- Luminosity : the amount of overall activity in an event is strongly correlated with the instantaneous luminosity. As the instantaneous luminosity increases, the probability of an additional inelastic interaction increases and more energy is deposited in the calorimeters and hence in the knockout region.

- $\boldsymbol{U}_{\|}$: in events where there is a large asymmetry in the momenta of the decay leptons, the transverse momentum of the $\mathrm{W}$ boson is in the direction of the highest $p_{T}$ lepton so the 'hard' QCD jet balancing it is in the opposite direction. This means that the recoil vector can point in the direction of the electron or muon depositing more energy in the knockout region. $U_{\|}$is the recoil projected in the direction of the lepton such that $U_{\|}>0$ if the recoil is parallel to the lepton and $U_{\|}<0$ if the recoil 
is in the antiparallel direction. The recoil energy in the knockout region therefore also has a dependence on $U_{\|}$.

- Tower $\boldsymbol{\eta}$ : the amount of energy deposited in the calorimeter also has a small dependence on the $\eta$ of the calorimeter tower. This dependence results from a difference in the size of the towers. The towers at high values of $|\eta|$ tend to be smaller and contain less energy.

The amount of recoil energy that needs to be subtracted from the recoil in the simulation is determined by studying $\mathrm{W} \rightarrow l \nu$ data. For each event in the $\mathrm{W} \rightarrow l \nu$ sample, pseudo knockout clusters are defined in the region $\Delta \phi= \pm 90^{\circ}$ from the charged lepton such that there is negligible bias from the 'hole' left by the knockout as well as background events which tend to populate the region $\Delta \phi= \pm 180^{\circ}$ from the lepton. The pseudo-clusters contain the same number of towers as the lepton knockout region and the central tower of the cluster has the same value of $\eta$ as the lepton. The energy in each cluster is studied as a function of luminosity, $U_{\|}$and $\eta$, where $U_{\|}$is redefined as the recoil vector projected in the direction of the pseudo-cluster. The distributions are shown for the $\mathrm{W}$ mass and width analyses in Figure 7.8.

For each event in the simulation, the energy distribution of the pseudo-clusters obtained from $\mathrm{W} \rightarrow l \nu$ events is sampled. This distribution is shown for the total electromagnetic and hadronic energy in Figure 7.8(a). The bin at -4.0 represents events for which the recoil energy in the pseudo-cluster is zero. For the $\mathrm{W}$ width analysis, $56 \%$ of events have no overlapping energy in the knockout region and for the $\mathrm{W}$ mass analysis $27 \%$ of events are in this zero bin.

Figure 7.8(b) shows the dependence on $U_{\|}$. At large positive $U_{\|}$the recoil vector is in the direction of the pseudo-cluster resulting in more energy. The slight rise in energy as $U_{\|}$becomes large and negative is due to a general increase in the recoil in the event so 


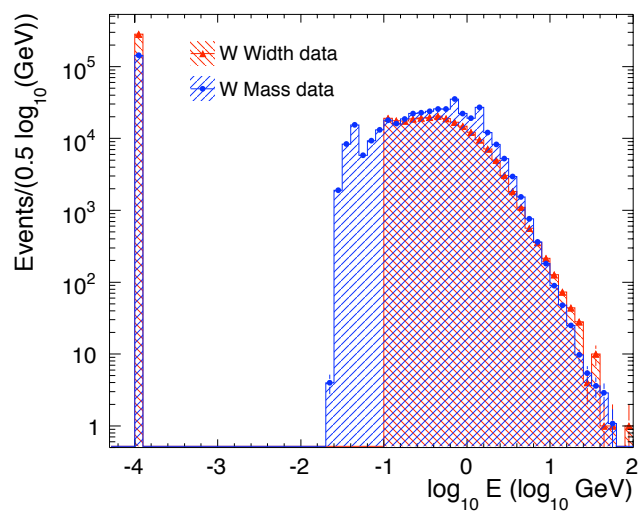

(a)

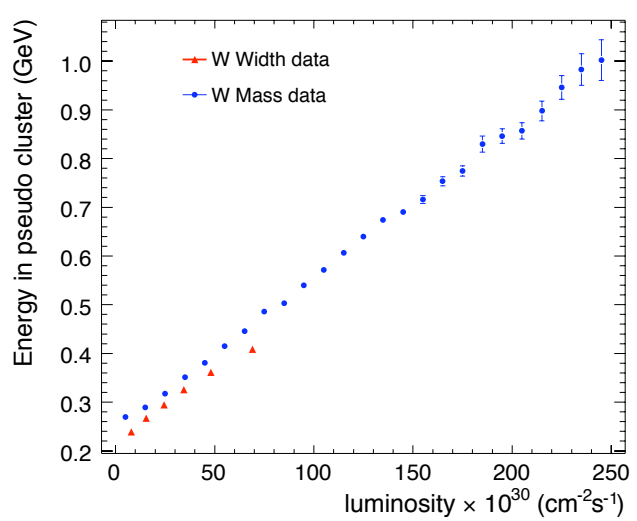

(c)

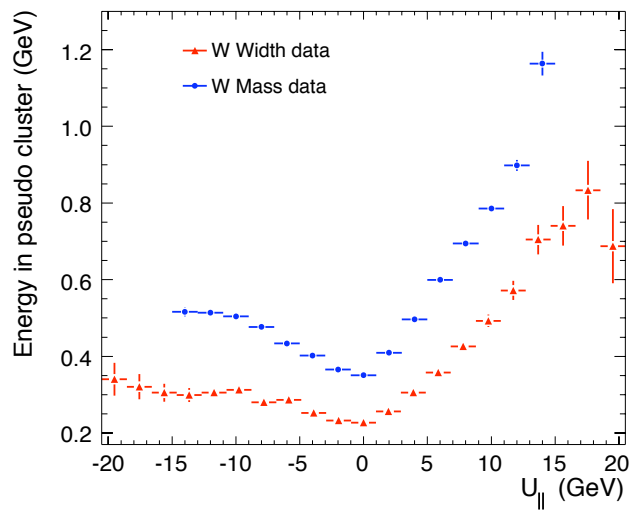

(b)

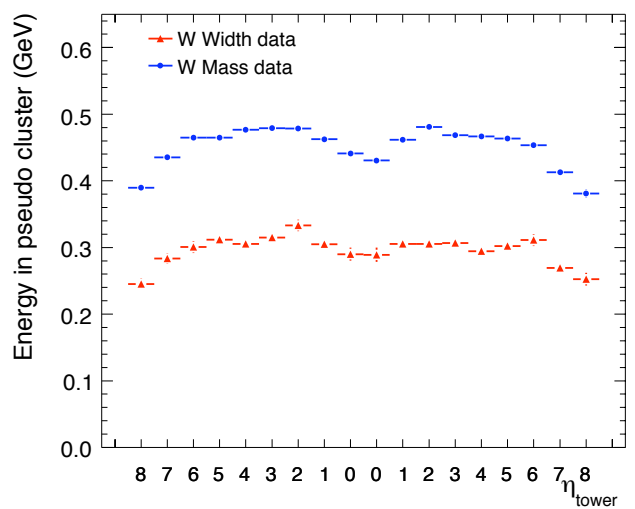

(d)

Figure 7.8: Underlying energy in a 7-tower pseudo-cluster in a region orthogonal in $\phi$ but at the same $\eta$ as the electron in $\mathrm{W} \rightarrow e \nu$ events shown in (a) as $\log _{10} E$, (b) as a function of $U_{\|}$, (c) as a function of instantaneous luminosity, (d) as a function of tower $\eta$.

there is on average more energy in the calorimeter. The value of the recoil cut in the W mass (width) analysis is $15 \mathrm{GeV}(20 \mathrm{GeV})$ and this results in $\left|U_{\|}\right|<15(20) \mathrm{GeV}$.

Figure 7.8(c) shows the cluster energy as a function of luminosity and a linear dependence is observed.

Figure 7.8(d) shows the dependence on the $\eta$ of the calorimeter tower. There is a small decrease in the energy at higher $\eta$ values because these towers are smaller in $\eta$. The dip in energy for the towers at small $\eta$ is also due to smaller tower size.

The dependence of the cluster energy on these variables is parameterised by fits to 
these distributions. The sampled cluster energy is then scaled based on multiplicative factors obtained from the dependence on $U_{\|}\left(S_{U_{\|}}\right)$, luminosity $\left(S_{\text {lumi }}\right)$ and $\eta\left(S_{\eta}\right)$ where

$$
\begin{aligned}
& S_{u_{\|}}=a_{0}+a_{1} \times\left(\frac{U_{\|}-\left|U_{\|}\right|}{2 U_{\|}}\right)+a_{2} \times\left(\frac{U_{\|}+\left|U_{\|}\right|}{2 U_{\|}}\right) \quad(\text { W Width }) \\
& S_{u_{\|}}=a_{0}+a_{1} \times\left(\frac{U_{\|}-\left|U_{\|}\right|}{2 U_{\|}}\right)+a_{2} \times\left(\frac{U_{\|}+\left|U_{\|}\right|}{2 U_{\|}}\right)+a_{3} \times\left(\frac{U_{\|}+\left|U_{\|}\right|}{2 U_{\|}}\right) U_{\|} \text {(W Mass) } \\
& S_{\text {lumi }}=b_{0}+b_{1} \times \text { lumi } \\
& S_{\eta}=c_{0}\left(\eta_{\text {tower }} \leq 6\right) ; S_{\eta}=c_{1}+c_{2} \times \eta\left(\eta_{\text {tower }}>6\right)
\end{aligned}
$$

The parameters $a_{0,1,2,3}, b_{0,1}$ and $c_{0,1,2}$ are obtained from fitting the above functional forms to the distributions in Figure 7.8. In general there is some correlation between $U_{\|}$ and luminosity. This is particularly evident for the large luminosity range of the W mass event samples. It is accounted for by studying the cluster energy as a function of $U_{\|}$in nine bins of luminosity to obtain parameters for the scale factors that decorrelate the two effects. This is shown for two bins of luminosity in Figure 7.9.

The recoil energy in the knockout region obtained by this method is subsequently subtracted from the recoil vector in the simulation which is obtained as described in Sections 7.2.2 and 7.2.3.

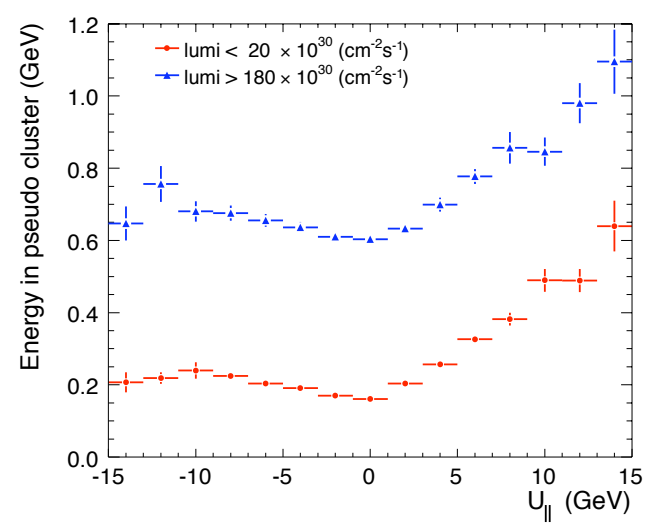

Figure 7.9: Underlying energy in a pseudo-cluster as a function of $U_{\|}$in two ranges of luminosity in $\mathrm{W} \rightarrow e \nu$ events for the $\mathrm{W}$ mass analysis. 


\subsubsection{Simulation of $U_{\mathrm{BREM}}$}

As noted earlier, the bremsstrahlung contribution to the recoil is simulated by adding to $U_{\mathrm{BREM}}$ the energies of the photons in the simulation that do not end up in the knockout region. Some of the towers that contribute to $U_{\mathrm{BREM}}$ are shown in Figure 7.7. The distribution of $U_{\mathrm{BREM}}$ obtained from the fast simulation for $\mathrm{W} \rightarrow e \nu$ and $\mathrm{W} \rightarrow \mu \nu$ events in the $\mathrm{W}$ mass analysis is shown in Figure 7.10. In $\mathrm{W} \rightarrow e \nu$ sample approximately $91 \%$ of events have $U_{\mathrm{BREM}}=0$ and in $\mathrm{W} \rightarrow \mu \nu$ events $94 \%$ have $U_{\mathrm{BREM}}=0$. The mean value of $U_{\mathrm{BREM}}$ for the remaining events is $860 \mathrm{MeV}$ for $\mathrm{W} \rightarrow e \nu$ and $1.31 \mathrm{GeV}$ for $\mathrm{W} \rightarrow \mu \nu$ events. This difference is due to the smaller knockout region for muons which means $U_{\text {BREM }}$ is calculated over a larger phase space.

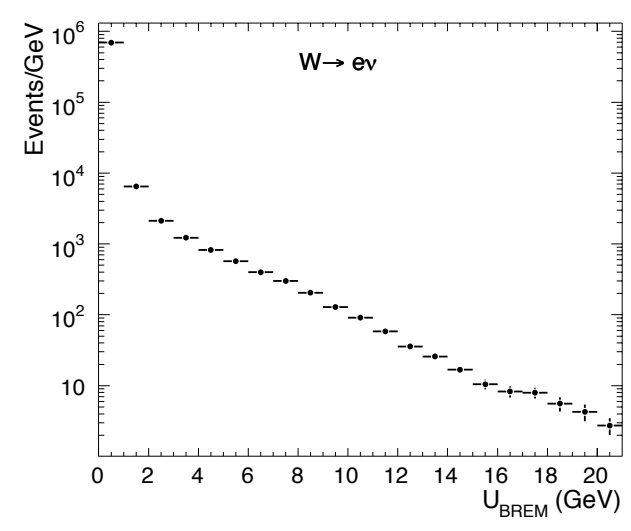

(a)

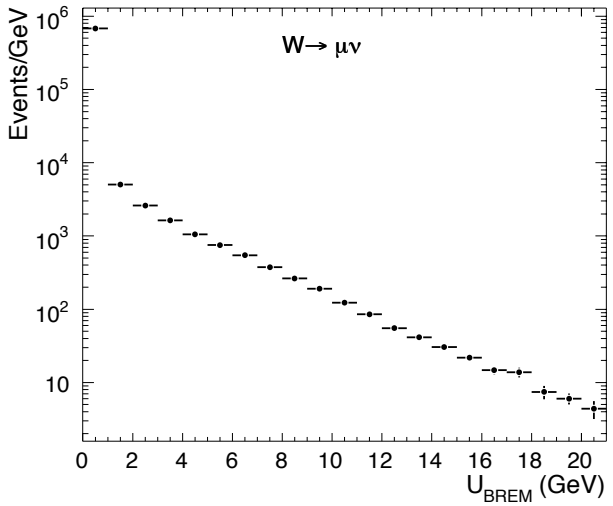

(b)

Figure 7.10: The distribution of the bremsstrahlung contribution to the recoil for $\mathrm{W} \rightarrow e \nu$ and $\mathrm{W} \rightarrow \mu \nu$ simulation events in the $\mathrm{W}$ mass analysis.

\subsubsection{The Recoil Model}

The other contributions, from initial state gluon radiation and underlying energy are simulated by an ad-hoc parametric model where the parameters are obtained by fitting to data. The magnitude and direction of the gluon radiation is expected to be strongly correlated with the transverse momentum of the boson. The $p_{\mathrm{T}}$ of the $\mathrm{Z}$ boson is well 
measured in $\mathrm{Z} \rightarrow l^{+} l^{-}$events and fits are performed to the recoil in these samples to obtain the model parameters.

In doing so, it is assumed that the hadronisation of the initial state gluon radiation is the same in $\mathrm{W}$ and $\mathrm{Z}$ events with the only difference arising from the variance in the $\mathrm{W}$ and $\mathrm{Z} p_{T}$ distributions. In the $\mathrm{W}$ width analysis, it is also assumed that there is a negligible bias from the difference in event topology of $\mathrm{Z}$ events, where both decay leptons are reconstructed in the central region of the calorimeter (referred to as CC events), and W events where the central requirement can only be made on the charged lepton. For the $\mathrm{W}$ mass measurement, this assumption is studied in detail in $\mathrm{Z} \rightarrow e^{+} e^{-}$events where one electron is reconstructed in the central calorimeter and one in the plug calorimeter (referred to as $\mathrm{CP}$ events) and compared to the recoil in CC Z events. This study is the subject of the next chapter.

The underlying energy contribution to the recoil is predominantly dependent on the instantaneous luminosity and hence the total scalar transverse energy in the event, $\Sigma E_{\mathrm{T}}$. This contribution is parameterised by fits to minimum-bias data.

The recoil vector can be projected into a number of directions, with some offering more information than others and dependent on different aspects of the simulation. The choice of projection can probe the different processes producing the recoil to varying extents. For the recoil model used in the $\mathrm{W}$ mass and width measurements, the direction of the $\mathrm{Z} p_{T}$ is used to project the recoil along two directions, in the direction of the boson $p_{T}\left(U_{1}\right)$ and the direction perpendicular (in the anticlockwise direction) to the boson $p_{T}\left(U_{2}\right)$ in the $x y$ plane. This projection is chosen to attempt to disentangle the hard QCD component from the soft underlying energy component. $U_{1}$ probes the QCD radiation which is emitted in the opposite direction to the boson $p_{T}$ and $U_{2}$ is largely sensitive to the soft (underlying energy) component.

Using the two components of this projection, the construction of the parametric model for the recoil is described in the following paragraphs. 
$U_{1}$ is effectively a measure of the calorimeter response to the initial state gluon radiation. It is defined as positive along the boson $p_{\mathrm{T}}$ direction and negative in the antiparallel direction. The mean of $U_{1}$ is therefore expected to become more and more negative with increasing boson $p_{\mathrm{T}}$. The soft underlying energy is expected to be azimuthally symmetric with no preferred direction and the mean of $U_{2}$ is therefore predicted to be zero.

The $U_{1}$ and $U_{2}$ components can be parameterised as

$$
\begin{gathered}
U_{1}=G\left(\left\langle U_{1}\right\rangle, \sigma\left(U_{1}\right)\right) \\
U_{2}=G\left(\left\langle U_{2}\right\rangle, \sigma\left(U_{2}\right)\right), \text { where }\left\langle U_{2}\right\rangle=0.0
\end{gathered}
$$

where $G$ represents a Gaussian distributed random variable with a mean $\left\langle U_{1}\right\rangle$ and variance $\sigma\left(U_{1}\right)$ for $U_{1}$ and mean of zero and variance $\sigma\left(U_{2}\right)$ for $U_{2}$. The functional form used to describe $\left\langle U_{1}\right\rangle$ in the $\mathrm{W}$ width analysis is given by

$$
\left\langle U_{1}\right\rangle=\left(P_{1}+P_{2} \times p_{T}\right) \times\left(1-e^{-P_{3} p_{T}}\right) \quad(\text { W Width })
$$

where $p_{T}$ is the true, unsmeared transverse momentum of the boson, since this is the only information that is unambiguously determined in the simulation for $\mathrm{W} \rightarrow l \nu$ events. The parameters $P_{1,2,3}$ are referred to as the $U_{1}$ response parameters and are obtained by fitting to the dependence of $\left\langle U_{1}\right\rangle$ on boson $p_{T}$ in $\mathrm{Z} \rightarrow l^{+} l^{-}$data.

In the $\mathrm{W}$ mass analysis, an alternative functional form for $\left\langle U_{1}\right\rangle$ taken from [22] is chosen which better decorrelates the response parameters. The following functional form described by 2 parameters is used

$$
\left\langle U_{1}\right\rangle=-P_{1} p_{T} \ln \left(p_{T}+P_{2}\right) / \ln \left(15+P_{2}\right) \quad \text { (W Mass). }
$$

The resolution of $U_{1}$ is dominated by the resolution of the QCD jet balancing the boson $p_{T}$, although it also receives a contribution from the soft underlying energy that lies in the $U_{1}$ direction. The resolution of $U_{2}$ is dominated by the underlying energy resolution 
with a small contribution from the 'hard' QCD component, in particular for events where the initial state radiation is not well collimated and where more than one gluon is emitted resulting in more than one jet in the final state.

The resolutions of $U_{1}$ and $U_{2}$ are parameterised as

$$
\begin{aligned}
& \sigma\left(U_{1}\right)=\sigma_{M B}\left(P_{4}+P_{5} \times p_{T}\right) \\
& \sigma\left(U_{2}\right)=\sigma_{M B}\left(P_{6}+P_{7} \times p_{T}\right)
\end{aligned}
$$

where $\sigma_{M B}$ is a description of the underlying energy resolution obtained from fits to minimum-bias data. This resolution is parameterised as a function of the $\Sigma E_{\mathrm{T}}$ of the event sample. The simulation of the $\Sigma E_{\mathrm{T}}$ distribution and the fits to minimum-bias data are described below.

\section{Parameterisation of $\Sigma E_{T}$}

The $\Sigma E_{\mathrm{T}}$ distribution is calculated in the data in the same way as the recoil albeit it is a scalar sum over the energies in the calorimeter towers. It has a dependence on the instantaneous luminosity in the event and the boson $p_{\mathrm{T}}$.

In the $\mathrm{W}$ width analysis, the $350 \mathrm{pb}^{-1}$ dataset has a small range in luminosity so a fit to the inclusive $\Sigma E_{\mathrm{T}}$ distribution gives an implicit luminosity dependence that is adequate for the data sample. An explicit dependence on the boson $p_{\mathrm{T}}$ is built into the $\Sigma E_{\mathrm{T}}$ functional form which is described by the following

$$
\Sigma E_{\mathrm{T}}=b \times \Gamma(c-1)
$$

where

$$
\begin{aligned}
b & =Q_{1}+Q_{2} \times p_{\mathrm{T}} \\
c & =Q_{3}+Q_{4} \times p_{\mathrm{T}}
\end{aligned}
$$


and $\Gamma$ is a Gamma distribution.

The parameters $Q_{1}$ to $Q_{4}$ are obtained from fits to the inclusive $\Sigma E_{\mathrm{T}}$ distribution and the $\Sigma E_{\mathrm{T}}$ distribution in bins of boson $p_{T}$. This is done separately for $\mathrm{Z} \rightarrow e^{+} e^{-}$ and $\mathrm{Z} \rightarrow \mu^{+} \mu^{-}$events to account for the different luminosity profiles of the two samples. The best fit $\Sigma E_{\mathrm{T}}$ parameters obtained are shown in Table 7.2 for the electron and muon channel. The fit results are shown in Figure 7.11 and the variation of $\left\langle\Sigma E_{\mathrm{T}}\right\rangle$ with $p_{\mathrm{T}}$ is shown in Figure 7.12. The inclusive $\Sigma E_{\mathrm{T}}$ distribution is well described in both the electron and muon channels. However, the variation of $\left\langle\Sigma E_{\mathrm{T}}\right\rangle$ with boson $p_{\mathrm{T}}$ is not well described, particularly in the low $p_{\mathrm{T}}$ region. For all subsequent plots, the data is represented by blue points and the simulation is denoted by a red histogram.

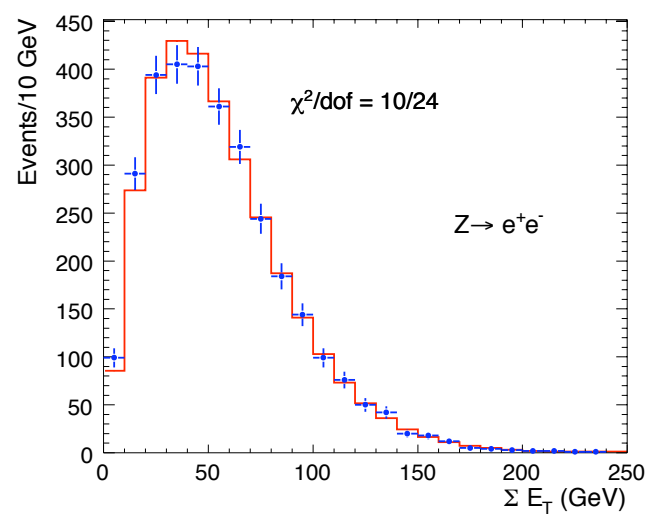

(a)

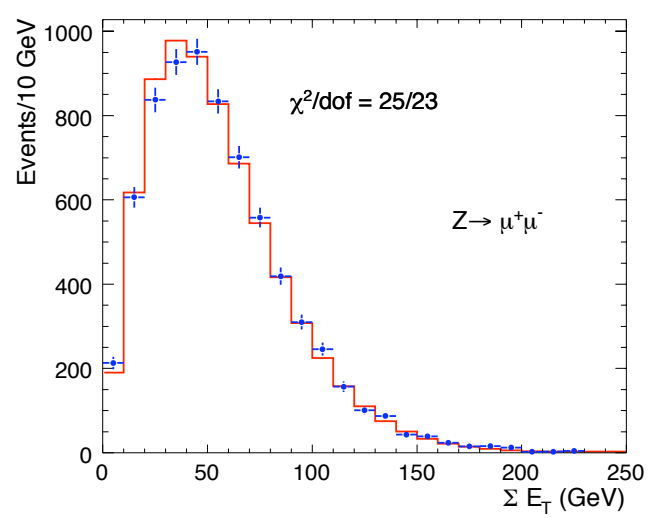

(b)

Figure 7.11: $\Sigma E_{\mathrm{T}}$ distributions for (a) $\mathrm{Z} \rightarrow e^{+} e^{-}$and (b) $\mathrm{Z} \rightarrow \mu^{+} \mu^{-}$data compared to the simulation using the best fit $\Sigma E_{\mathrm{T}}$ parameters in the $\mathrm{W}$ width analysis.

\begin{tabular}{|c|cc|}
\hline & $\mathrm{Z} \rightarrow e^{+} e^{-}$ & $\mathrm{Z} \rightarrow \mu^{+} \mu^{-}$ \\
\hline \hline$Q_{1}$ & 20.700 & 19.351 \\
$Q_{2}$ & -0.154 & -0.141 \\
$Q_{3}$ & 1.055 & 1.051 \\
$Q_{4}$ & 0.085 & 0.100 \\
\hline
\end{tabular}

Table 7.2: The best fit $\Sigma E_{\mathrm{T}}$ parameters obtained by fitting $\mathrm{Z} \rightarrow e^{+} e^{-}$and $\mathrm{Z} \rightarrow \mu^{+} \mu^{-}$events in the W width analysis.

The instantaneous luminosity distribution for $\mathrm{Z} \rightarrow e^{+} e^{-}$events in the $\mathrm{W}$ mass and 


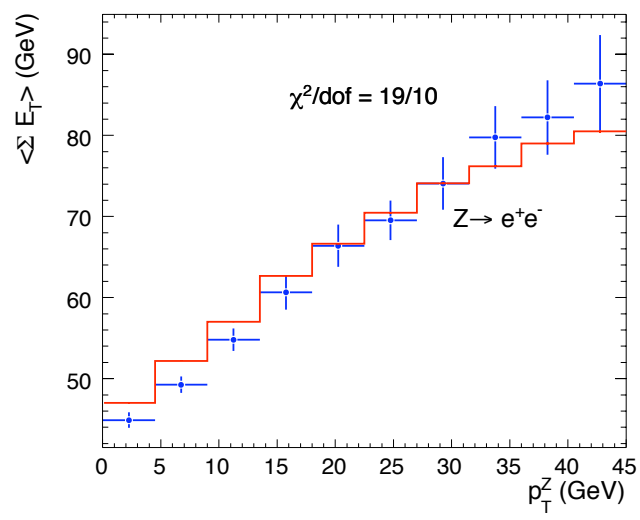

(a)

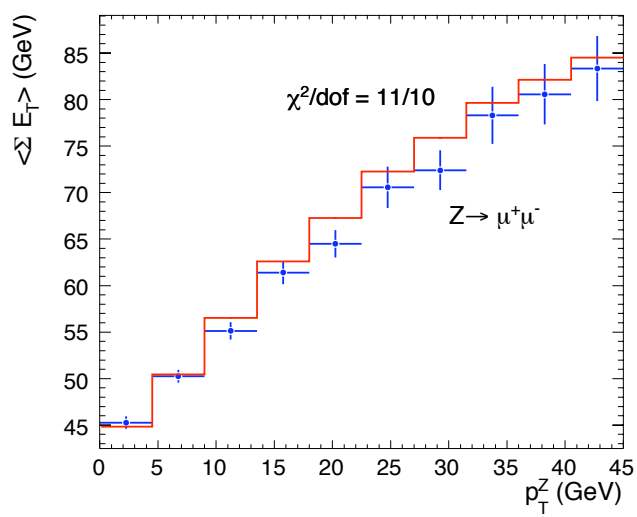

(b)

Figure 7.12: The variation of $\left\langle\Sigma E_{\mathrm{T}}\right\rangle$ with the transverse momentum of the $\mathrm{Z}$ boson in data and simulation for (a) $\mathrm{Z} \rightarrow e^{+} e^{-}$events and (b) $\mathrm{Z} \rightarrow \mu^{+} \mu^{-}$events.

width measurements are shown in Figure 7.13. In proceeding from the $\mathrm{W}$ width measurement to the $\mathrm{W}$ mass measurement, the average instantaneous luminosity of the datasets increased from approximately $27 \times 10^{30} \mathrm{~cm}^{-2} \mathrm{~s}^{-1}$ to $70 \times 10^{30} \mathrm{~cm}^{-2} \mathrm{~s}^{-1}$. The increased range in luminosity for the $\mathrm{W}$ mass dataset requires the inclusion of an explicit luminosity dependence into the recoil model. This is achieved by constructing a parameterisation for the $\Sigma E_{\mathrm{T}}$ that is dependent on both instantaneous luminosity and boson $p_{\mathrm{T}}$.

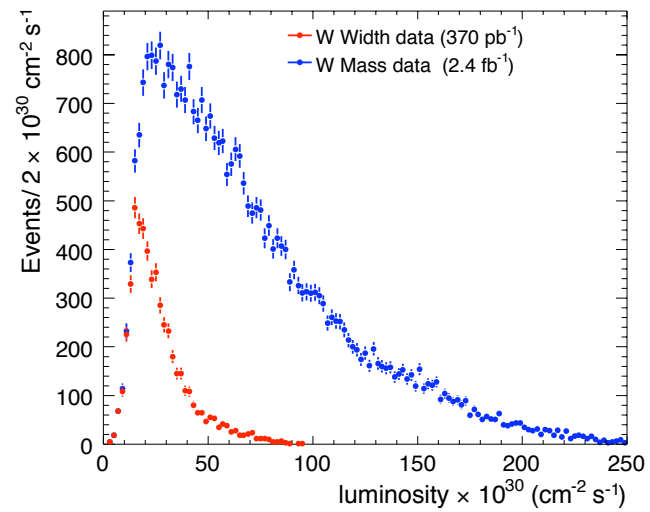

Figure 7.13: Comparison of the instantaneous luminosity distribution in $\mathrm{Z} \rightarrow e^{+} e^{-}$events for the 370 $\mathrm{pb}^{-1}$ data sample used in the $\mathrm{W}$ width analysis and the $2.4 \mathrm{fb}^{-1}$ data sample used in the $\mathrm{W}$ mass analysis.

The $\Sigma E_{\mathrm{T}}$ is generated according to the gamma distribution as in the $\mathrm{W}$ width analysis. The mean and standard deviation of the gamma distribution are related to the parameters 
$b$ and $c$ in Equation 7.15 in the following way

$$
\begin{aligned}
& b=\frac{\left[\sigma\left(\Sigma E_{\mathrm{T}}\right)\right]^{2}}{\left\langle\Sigma E_{\mathrm{T}}\right\rangle} \\
& c=\frac{\left\langle\Sigma E_{\mathrm{T}}\right\rangle^{2}}{\left[\sigma\left(\Sigma E_{\mathrm{T}}\right)\right]^{2}}
\end{aligned}
$$

Simple fits to the $\Sigma E_{\mathrm{T}}$ distribution in bins of $p_{\mathrm{T}}$ and luminosity in the data (given in Figure 7.15) show that the mean of the $\Sigma E_{\mathrm{T}}$ distribution increases linearly with luminosity and boson $p_{\mathrm{T}}$. The mean and standard deviation of the $\Sigma E_{\mathrm{T}}$ are parameterised as

$$
\begin{gathered}
\left\langle\Sigma E_{\mathrm{T}}\right\rangle=a_{0}+a_{1} \times p_{\mathrm{T}}+a_{2} \times \mathcal{L} \\
\sigma\left(\Sigma E_{\mathrm{T}}\right)=b_{0}+b_{1} \times p_{\mathrm{T}}+b_{2} \times \mathcal{L}^{0.48}
\end{gathered}
$$

where $p_{\mathrm{T}}$ is the true boson $p_{\mathrm{T}}$ and the luminosity $(\mathcal{L})$ for a given event sample is obtained by sampling from the luminosity distribution in the data. A $\Sigma E_{\mathrm{T}}$ distribution is generated in the simulation according to Equation 7.15 and substituting Equations 7.20 and 7.21 into the definition of $b$ and $c$ defined above.

The parameters $a_{0,1,2}$ and $b_{0,1,2}$ are obtained by minimising the total $\chi^{2}$ from fits to four distributions; the inclusive $\Sigma E_{\mathrm{T}}$ distribution, $\left\langle\Sigma E_{\mathrm{T}}\right\rangle$ vs. $p_{\mathrm{T}},\left\langle\Sigma E_{\mathrm{T}}\right\rangle$ vs. luminosity, $\sigma\left(\Sigma E_{\mathrm{T}}\right)$ vs. $p_{\mathrm{T}}$ and $\sigma\left(\Sigma E_{\mathrm{T}}\right)$ vs. luminosity. The minimum $\chi^{2}$ obtained is $187 / 101$ and $127 / 101$ for $\mathrm{Z} \rightarrow e^{+} e^{-}$and $\mathrm{Z} \rightarrow \mu^{+} \mu^{-}$events respectively and the best fit parameters are shown in Table 7.3. The errors on the parameters are not shown as it was observed that varying the parameters by $1 \sigma$ does not affect the fitted mass of the $\mathrm{W}$ boson. The fit distributions are shown in Figure 7.14, 7.15 and 7.16. The distributions show that the luminosity and boson $p_{\mathrm{T}}$ dependence of the $\Sigma E_{\mathrm{T}}$ are not well modelled. A precise agreement between data and simulation for these distributions is not essential since $\Sigma E_{\mathrm{T}}$ enters into the definition of $\sigma_{M B}$ which enters into the functional forms of $\sigma\left(U_{1}\right), \sigma\left(U_{2}\right)$. The resolutions of $U_{1}$ and $U_{2}$ are fit for in the recoil fit so any minor mismodelling in the $\Sigma E_{\mathrm{T}}$ 
description is corrected in the $\sigma\left(U_{1}\right)$ and $\sigma\left(U_{2}\right)$ modelling. The effect of this mismodelling on the transverse mass is evaluated by taking the distribution that gives the worst agreement between data and simulation, $\sigma\left(\Sigma E_{\mathrm{T}}\right)$ vs. $p_{\mathrm{T}}$, and reweighting the $\Sigma E_{\mathrm{T}}$ distribution in each $p_{\mathrm{T}}$ bin such that perfect agreement is obtained between data and simulation. The recoil parameters obtained from fits to the recoil distributions with this reweighting are used to produce a transverse mass distribution. A fit to the transverse mass distribution gives a negligible shift in the $\mathrm{W}$ mass.

\section{Minimum-Bias Fits}

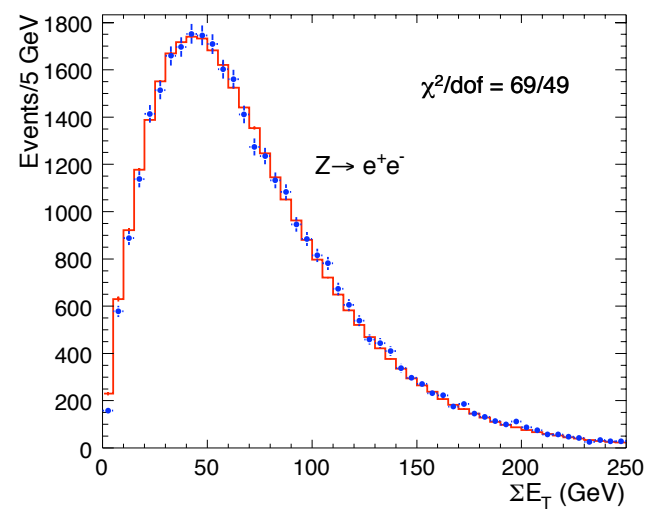

(a)

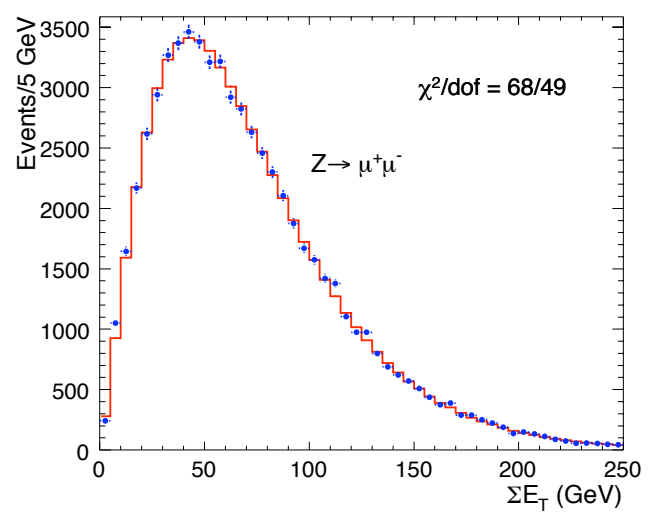

(b)

Figure 7.14: $\Sigma E_{\mathrm{T}}$ distributions for (a) $\mathrm{Z} \rightarrow e^{+} e^{-}$and (b) $\mathrm{Z} \rightarrow \mu^{+} \mu^{-}$data compared to the simulation using the best fit $\Sigma E_{\mathrm{T}}$ parameters in the $\mathrm{W}$ mass analysis.

\begin{tabular}{|c|cc|}
\hline & $\mathrm{Z} \rightarrow e^{+} e^{-}$ & $\mathrm{Z} \rightarrow \mu^{+} \mu^{-}$ \\
\hline \hline$a_{0}$ & 26.15 & 26.49 \\
$a_{1}$ & 1.110 & 1.127 \\
$a_{2}$ & 0.496 & 0.501 \\
$b_{0}$ & 12.54 & 12.61 \\
$b_{1}$ & 0.0560 & 0.0447 \\
$b_{2}$ & 3.452 & 3.463 \\
\hline
\end{tabular}

Table 7.3: The $\Sigma E_{\mathrm{T}}$ parameters obtained by fitting $\mathrm{Z} \rightarrow e^{+} e^{-}$and $\mathrm{Z} \rightarrow \mu^{+} \mu^{-}$events in the $\mathrm{W}$ mass analysis.

The parameters describing $\sigma_{M B}$ are obtained by fitting to the variance of the recoil in 

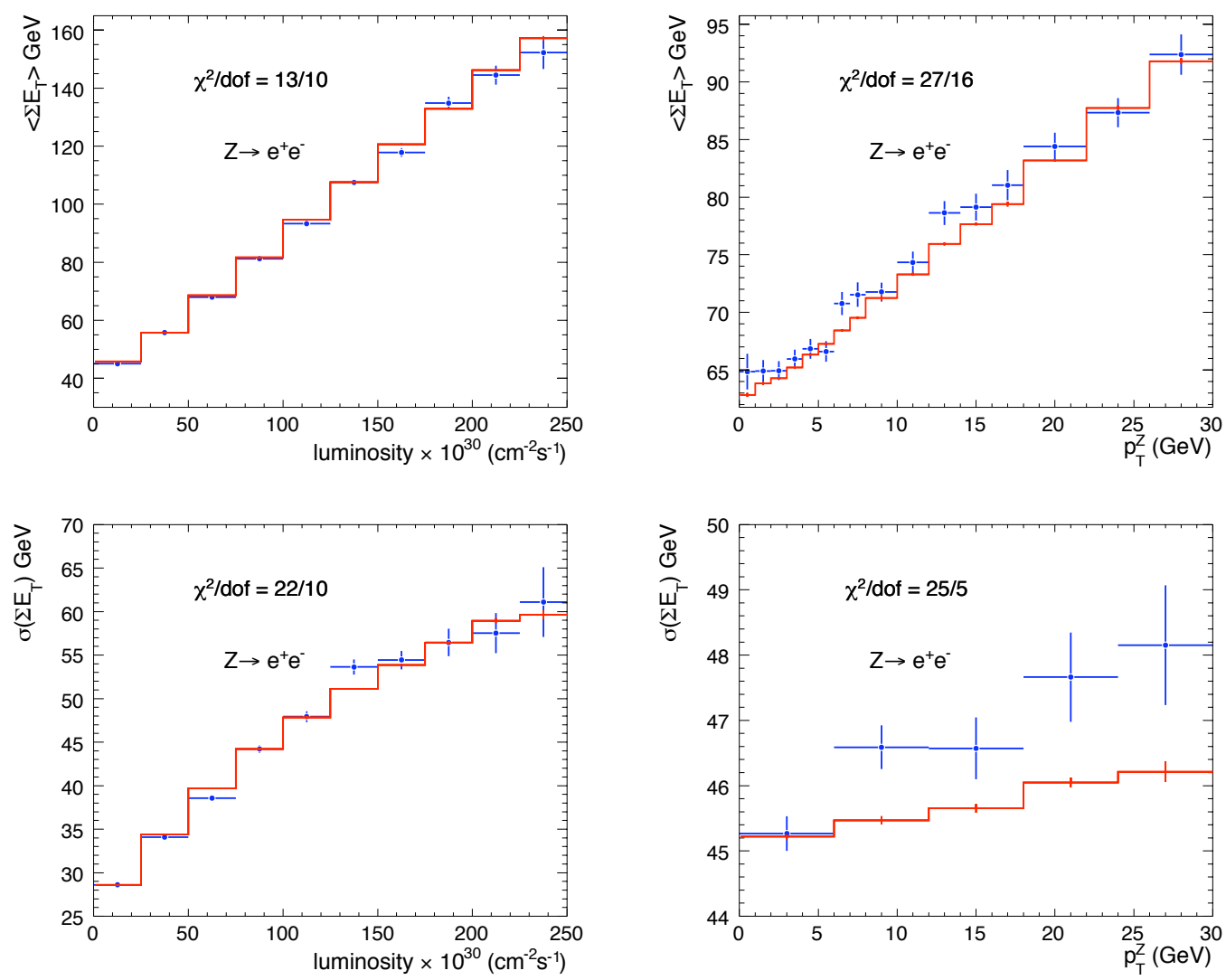

Figure 7.15: The dependence of the mean and standard deviation of the $\Sigma E_{\mathrm{T}}$ on luminosity and boson $p_{\mathrm{T}}$ in $\mathrm{Z} \rightarrow e^{+} e^{-}$events. These distributions are used in the fit to obtain the $\Sigma E_{\mathrm{T}}$ parameters for the electron channel.

minimum-bias events. The recoil projected along the $x$ and $y$ axes, $U_{x}$ and $U_{y}$ is plotted in bins of the $\Sigma E_{\mathrm{T}}$ in minimum-bias events. The following function is fitted to the resolution of the $U_{x}$ and $U_{y}$ distributions against $\Sigma E_{\mathrm{T}}$

$$
\sigma\left(U_{x, y}\right)=M_{1} \times \Sigma E_{\mathrm{T}}{ }^{M_{2}}
$$

In the $\mathrm{W}$ width analysis, the parameters $M_{1}$ and $M_{2}$ are found to be $M_{1}=0.338 \pm 0.002$, $M_{2}=0.559 \pm 0.002$ from a combined fit to the $U_{x}$ and $U_{y}$ resolutions. The resolution is therefore similar to the CEM resolution in Equation 6.10 and increases approximately proportionally with the square root of the total calorimeter $E_{T}$. The fits are shown in Figure 7.17 . 


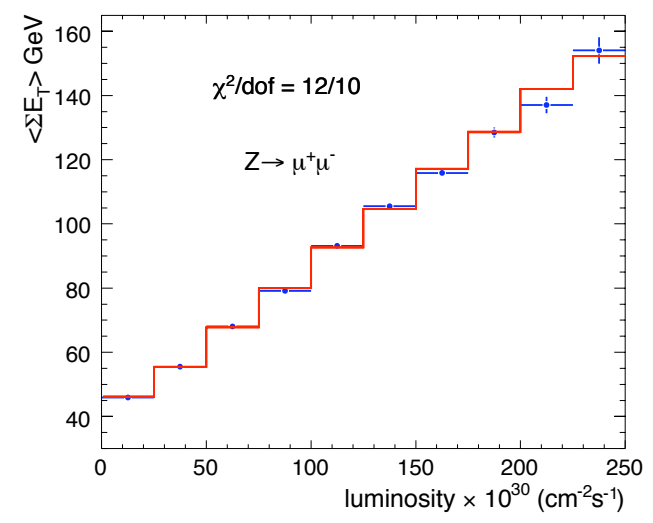

(a)

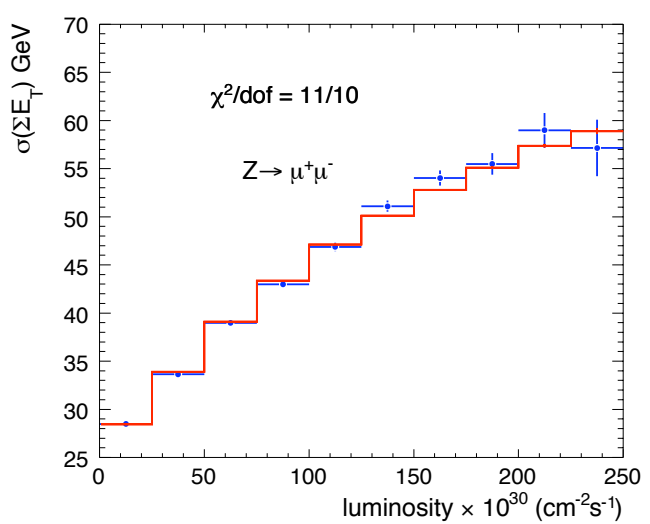

(c)

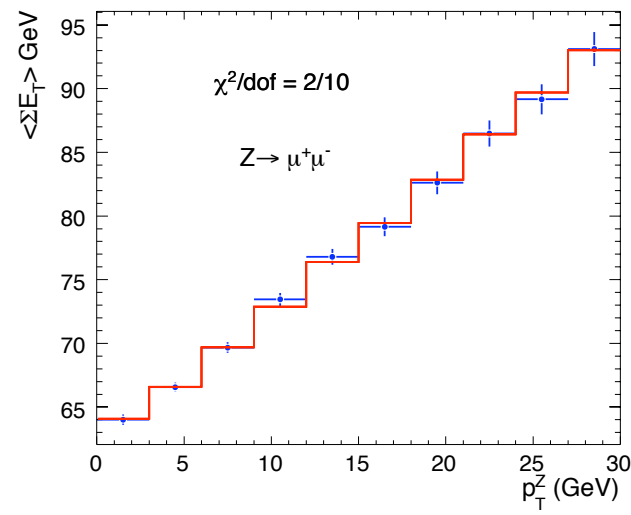

(b)

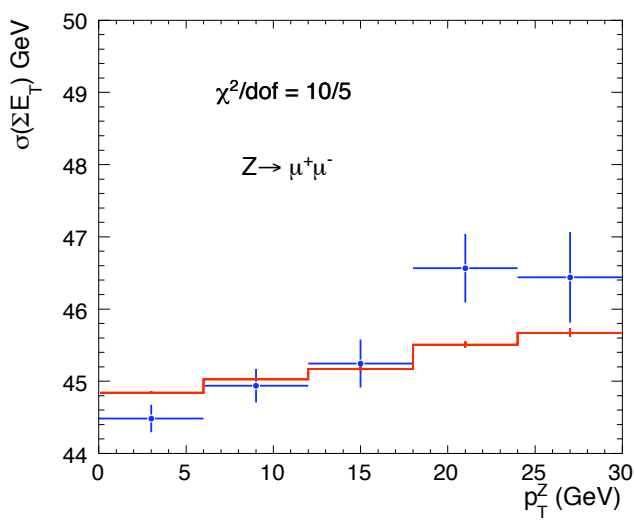

(d)

Figure 7.16: The dependence of the mean and standard deviation of the $\Sigma E_{\mathrm{T}}$ on luminosity and boson $p_{\mathrm{T}}$ in $\mathrm{Z} \rightarrow \mu^{+} \mu^{-}$events. These distributions are used in the fit to obtain the $\Sigma E_{\mathrm{T}}$ parameters for the muon channel.

In the $\mathrm{W}$ mass analysis, it was found that a better description of the resolution in minimum-bias data could be obtained by fitting separately the low $\Sigma E_{\mathrm{T}}$ region $\left(\Sigma E_{\mathrm{T}}\right.$ $<30 \mathrm{GeV})$ and the high $\Sigma E_{\mathrm{T}}$ region $\left(\Sigma E_{\mathrm{T}}>30 \mathrm{GeV}\right)$ and using the following three parameter functional form

$$
\sigma\left(U_{x, y}\right)=M_{1} \times \Sigma E_{\mathrm{T}}^{M_{2}}+M_{3} .
$$

The results of the fit are shown in Figure 7.18. The best fit parameters obtained are $M_{1}=0.20 \pm 0.02, M_{2}=0.70 \pm 0.03, M_{3}=0.31 \pm 0.04$ for the region $\Sigma E_{\mathrm{T}}<30 \mathrm{GeV}$ and $M_{1}=0.58 \pm 0.06, M_{2}=0.47 \pm 0.01, M_{3}=-0.4 \pm 0.2$ for the region $\Sigma E_{\mathrm{T}}>30 \mathrm{GeV}$. 


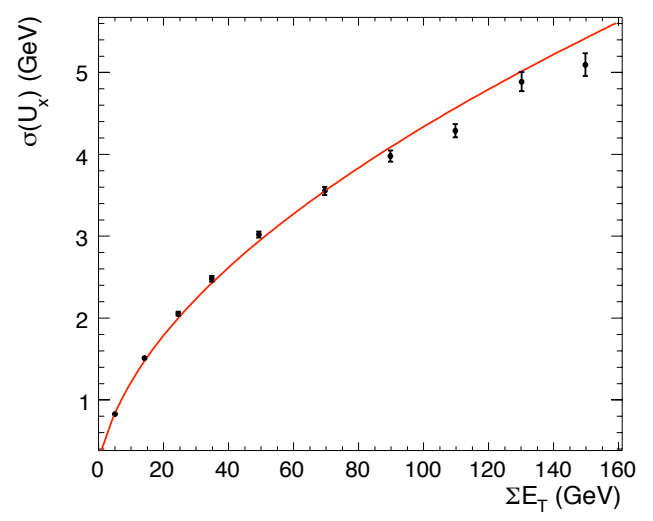

(a)

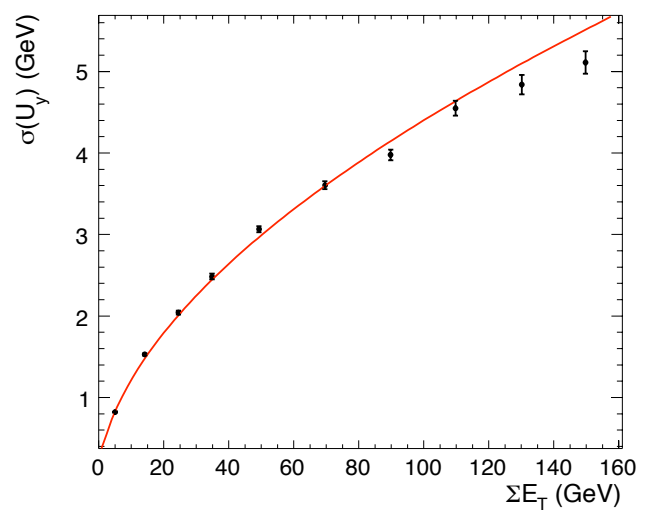

(b)

Figure 7.17: The fit to the (a) $\sigma\left(U_{x}\right)$ and (b) $\sigma\left(U_{y}\right)$ distributions as a function of $\Sigma E_{\mathrm{T}}$ in minimum-bias data for the $\mathrm{W}$ width analysis.

\subsubsection{Recoil Fits}

The recoil model described above is used to obtain a recoil vector in the simulation that is added to the recoil vector from the bremsstrahlung contribution. The recoil energy in the knockout region, as described in Section 7.2.1, is subsequently subtracted from this and the resulting recoil is then fitted to the recoil in the data. The parameters of the model are obtained by fitting to distributions in $\mathrm{Z} \rightarrow l^{+} l^{-}$data. The parameters $P_{1,2,3}$ are obtained by fitting to the variation of $\left\langle U_{1}\right\rangle$ as a function of boson $p_{\mathrm{T}}$. This is done separately for the $\mathrm{Z} \rightarrow e^{+} e^{-}$and $\mathrm{Z} \rightarrow \mu^{+} \mu^{-}$samples.

Similarly, the parameters $P_{4,5}$ are obtained by fitting to the $\sigma\left(U_{1}\right)$ vs. boson $p_{\mathrm{T}}$ distribution and $P_{6,7}$ are obtained by fitting to the $\sigma\left(U_{2}\right)$ vs. $p_{\mathrm{T}}$ distribution. The fitted distributions are shown in Figure 7.19 for $\mathrm{Z} \rightarrow e^{+} e^{-}$and $\mathrm{Z} \rightarrow \mu^{+} \mu^{-}$channels in the W width analysis.

The calorimeter response to boson $p_{\mathrm{T}}$ drops to $60 \%$ at low $p_{\mathrm{T}}(<4 \mathrm{GeV})$ from losing very low $p_{\mathrm{T}}$ particles that are swept away by the magnetic field and do not make it to the calorimeter. At high $p_{\mathrm{T}}$, the calorimeter response is approximately 80\%. Figures 7.19(a) and 7.19(b) show that this variation in the response of $U_{1}$ with boson $p_{\mathrm{T}}$ is well modelled 


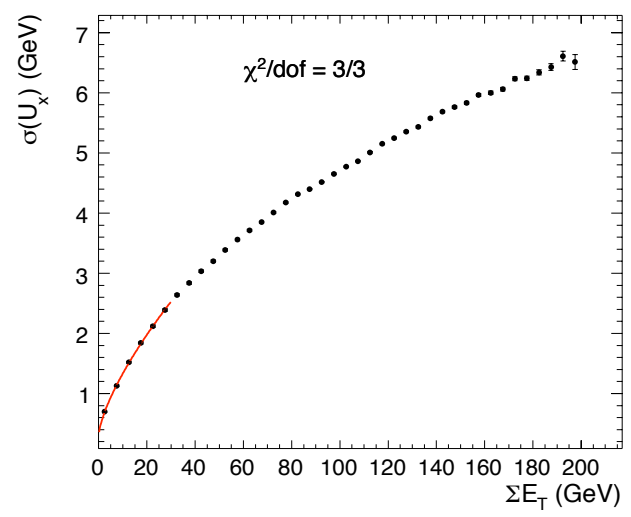

(a)

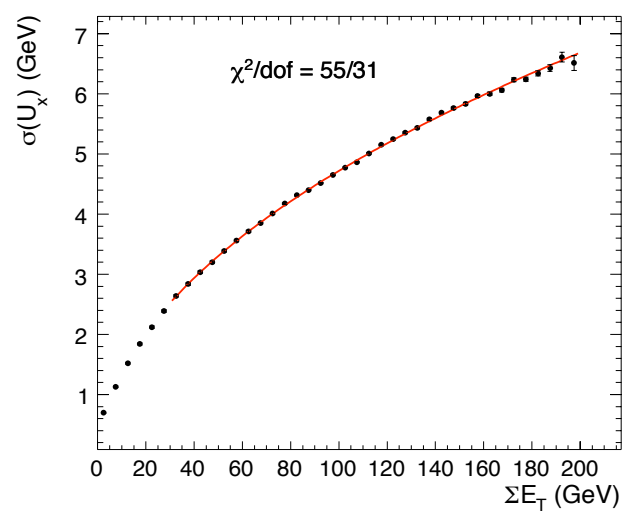

(c)

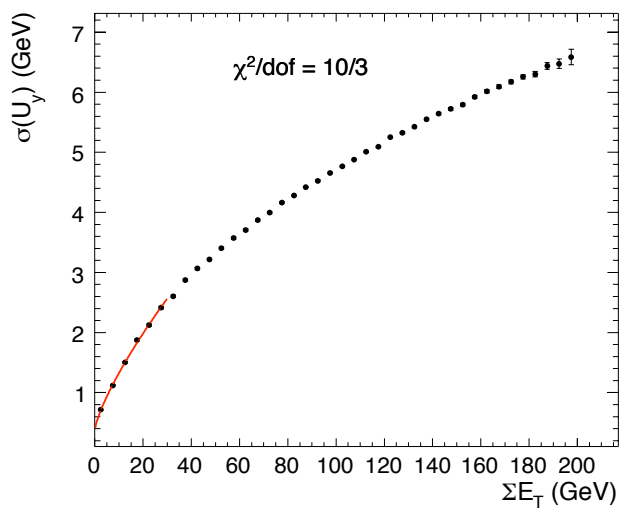

(b)

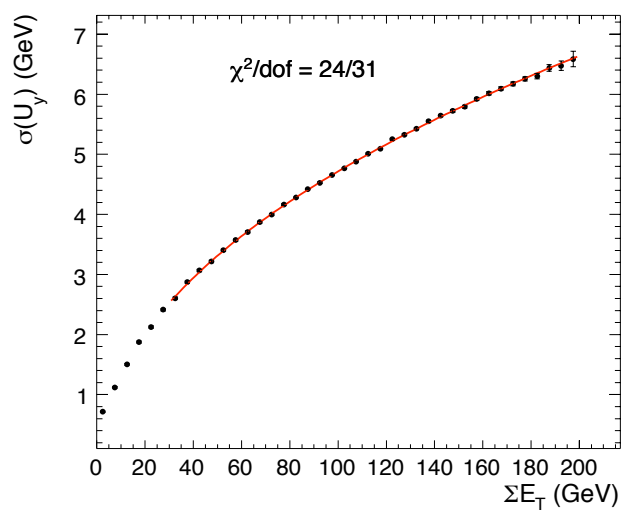

(d)

Figure 7.18: The fit to the (a) $\sigma\left(U_{x}\right)$ and (b) $\sigma\left(U_{y}\right)$ distributions as a function of $\Sigma E_{\mathrm{T}}$ in minimum-bias data for the $\mathrm{W}$ mass analysis.

by the simulation in $\mathrm{Z} \rightarrow e^{+} e^{-}$and $\mathrm{Z} \rightarrow \mu^{+} \mu^{-}$events respectively.

The distribution of $\sigma\left(U_{1}\right)$ vs. $p_{\mathrm{T}}$ shown in Figure 7.19(c) and Figure 7.19(d) is dominated at low boson $p_{\mathrm{T}}$ by the resolution on underlying energy and at high $p_{\mathrm{T}}$ by the 'hard' QCD radiation. The distribution is well described by the simulation. $U_{1}$ and $U_{2}$ have similar resolutions at very low $p_{\mathrm{T}}(<5 \mathrm{GeV})$, where the resolution is dominated by the underlying energy contribution $\sigma_{M B}$. Additional distributions that are used to monitor the quality of the recoil fits are shown in Figure 7.20.

The parameters obtained from fits to these distributions are shown in Table 7.4. In general, there are correlations between the three $U_{1}$ response parameters $P_{1,2,3}$ but very 
little correlation between the $U_{1}$ response and resolution parameters $\left(P_{4,5}\right)$ and almost no correlation between the $U_{1}$ and $U_{2}$ parameters.

\begin{tabular}{|l|rll|rll|}
\hline \multicolumn{7}{|c|}{ W Width } \\
\hline & \multicolumn{2}{|c|}{$\mathrm{Z} \rightarrow e^{+} e^{-}$} & \multicolumn{2}{|c|}{$\mathrm{Z} \rightarrow \mu^{+} \mu^{-}$} \\
\hline \hline$P_{1}$ & -12.7 & \pm & 1.1 & -13.1 & \pm & 1.5 \\
$P_{2}$ & -0.59 & \pm & 0.02 & -0.59 & \pm & 0.02 \\
$P_{3}$ & 0.043 & \pm & 0.003 & 0.042 & \pm & 0.004 \\
& & & & & & \\
$P_{4}$ & 0.93 & \pm & 0.03 & 0.97 & \pm & 0.02 \\
$P_{5}$ & 0.019 & \pm & 0.003 & 0.016 & \pm & 0.002 \\
& & & & & & \\
$P_{6}$ & 1.03 & \pm & 0.03 & 1.06 & \pm & 0.02 \\
$P_{7}$ & 0.0002 & \pm & 0.002 & -0.002 & \pm & 0.001 \\
\hline
\end{tabular}

Table 7.4: The best fit recoil parameters and their statistical uncertainty for $\mathrm{Z} \rightarrow e^{+} e^{-}$and $\mathrm{Z} \rightarrow \mu^{+} \mu^{-}$ events in the $\mathrm{W}$ width analysis.

In the $\mathrm{W}$ mass analysis, the variation of the $U_{1}$ response with boson $p_{\mathrm{T}}$ is described by two parameters. As mentioned, the functional form for the response is chosen to decorrelate the two parameters. Figure 7.23 shows the correlation contour between $P_{1}$ and $P_{2}$. They are largely decorrelated with a correlation coefficient of 0.37 .

The resolutions of $U_{1}$ and $U_{2}$ are described in the same way as in the $\mathrm{W}$ width analysis. The results of the fits are shown in Figure 7.21 for the electron and muon channels. The fits show that the distributions are well modelled by the simulation. The variation of $\sigma\left(U_{2}\right)$ with boson $p_{\mathrm{T}}$ in the electron channel shows a $3 \sigma$ discrepancy in the region $p_{\mathrm{T}}<$ $1 \mathrm{GeV}$. The effect of this disagreement on the $\mathrm{W}$ mass is evaluated by reweighting the distribution of $U_{2}$ for $p_{\mathrm{T}}<1 \mathrm{GeV}$ such that the simulation agrees well with the data. The distribution of $\sigma\left(U_{2}\right)$ vs. $p_{\mathrm{T}}$ is then fitted to obtain the parameters $P_{6,7}$. The $M_{T}$ distribution obtained using these parameters is subsequently fitted and the shift in the fitted $\mathrm{W}$ mass due to this disagreement in the low $p_{\mathrm{T}}$ region is found to be $5 \mathrm{MeV}$.

The luminosity dependence of the recoil model which was introduced in the $\Sigma E_{\mathrm{T}}$ parameterisation can be verified by studying the variation of the $U_{1}$ and $U_{2}$ resolutions 
as a function of luminosity. The distributions are shown in Figure 7.22. The dependence on luminosity is not well described. The effect of this discrepancy on the transverse mass is evaluated by reweighting the $U_{1}$ distribution in each luminosity bin such that the simulation gives a perfect description of the data. The recoil fits are performed to obtain the recoil parameters which are subsequently used to produce a transverse mass distribution. A shift in the $\mathrm{W}$ mass due to a discrepancy in these distributions is obtained by fitting to this $M_{T}$ distribution and is found to be $2.9 \mathrm{MeV}$ for the electron channel and $3.5 \mathrm{MeV}$ in the muon channel. Additional distributions that are used to monitor the quality of the recoil fits are shown in Figure 7.24. The parameters obtained from the fits to $\mathrm{Z} \rightarrow e^{+} e^{-}$and $\mathrm{Z} \rightarrow \mu^{+} \mu^{-}$data are given in Table 7.5 .

\begin{tabular}{|l|rll|rll|}
\hline \multicolumn{7}{|c|}{ W Mass } \\
\hline & $\mathrm{Z} \rightarrow e^{+} e^{-}$ & \multicolumn{2}{|c|}{$\mathrm{Z} \rightarrow \mu^{+} \mu^{-}$} \\
\hline \hline$P_{1}$ & 0.625 & \pm & 0.003 & 0.632 & \pm & 0.002 \\
$P_{2}$ & 9.5 & \pm & 0.9 & 9.0 & \pm & 0.6 \\
& & & & & & \\
$P_{3}$ & 0.971 & \pm & 0.009 & 0.963 & \pm & 0.006 \\
$P_{4}$ & 0.0105 & \pm & 0.0009 & 0.0106 & \pm & 0.0006 \\
& & & & & & \\
$P_{5}$ & 1.023 & \pm & 0.009 & 0.996 & \pm & 0.006 \\
$P_{6}$ & -0.0023 & \pm & 0.0008 & -0.0003 & \pm & 0.0005 \\
\hline
\end{tabular}

Table 7.5: The best fit recoil parameters and their statistical uncertainty for $\mathrm{Z} \rightarrow e^{+} e^{-}$and $\mathrm{Z} \rightarrow \mu^{+} \mu^{-}$ events in the $\mathrm{W}$ mass analysis. 


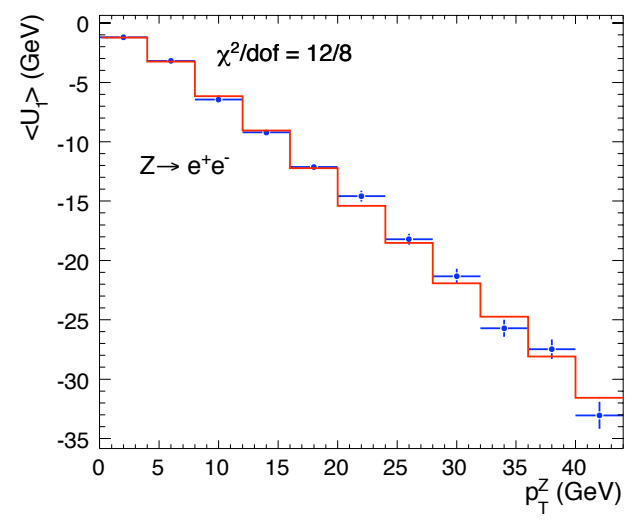

(a)

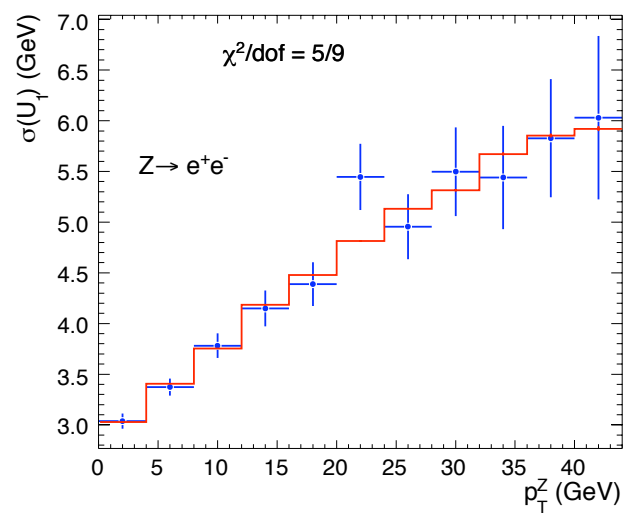

(c)

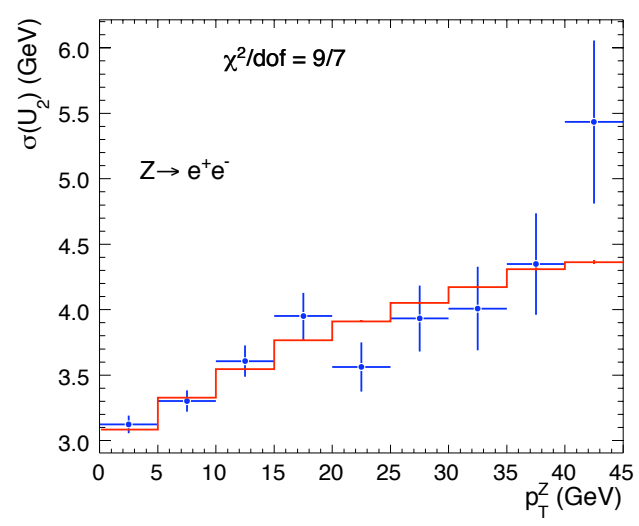

(e)

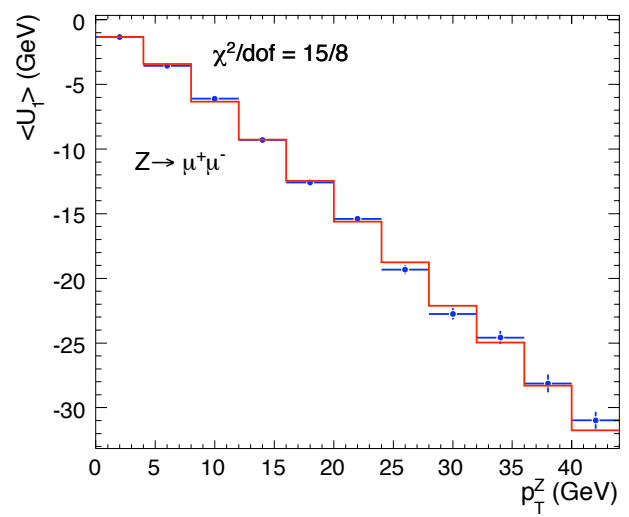

(b)

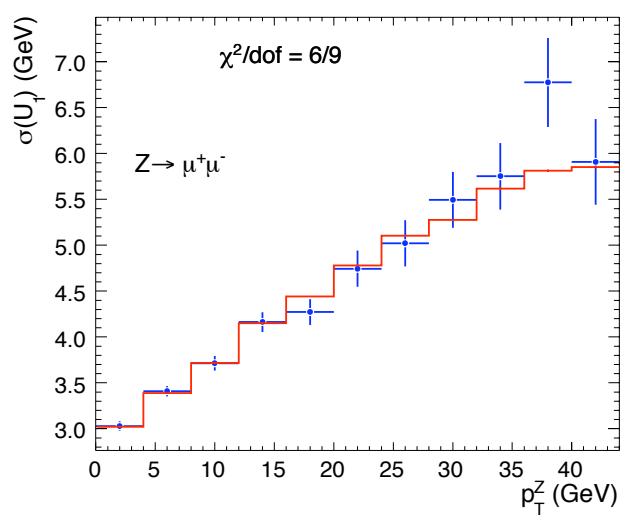

(d)

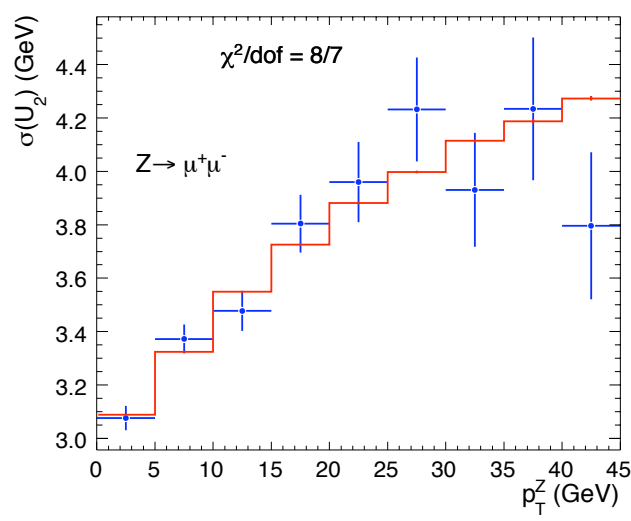

(f)

Figure 7.19: The distributions of (a) and (b) $\left\langle U_{1}\right\rangle$ vs. $p_{T}^{Z}$, (c) and (d) $\sigma\left(U_{1}\right)$ vs. $p_{T}^{Z}$, (e) and (f) $\sigma\left(U_{2}\right)$ vs. $p_{T}^{Z}$ in $\mathrm{Z} \rightarrow e^{+} e^{-}$and $\mathrm{Z} \rightarrow \mu^{+} \mu^{-}$events compared to the best fit simulation in the $\mathrm{W}$ width analysis. 


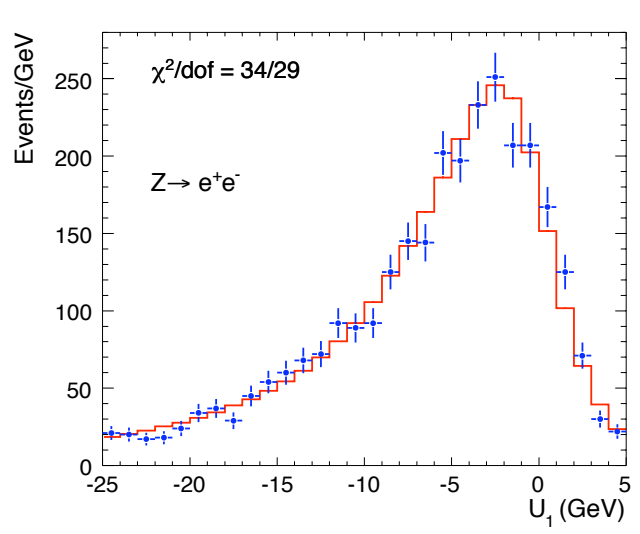

(a)

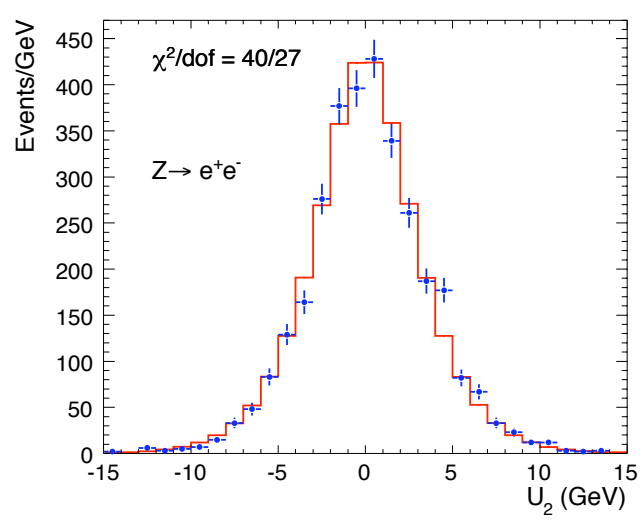

(c)

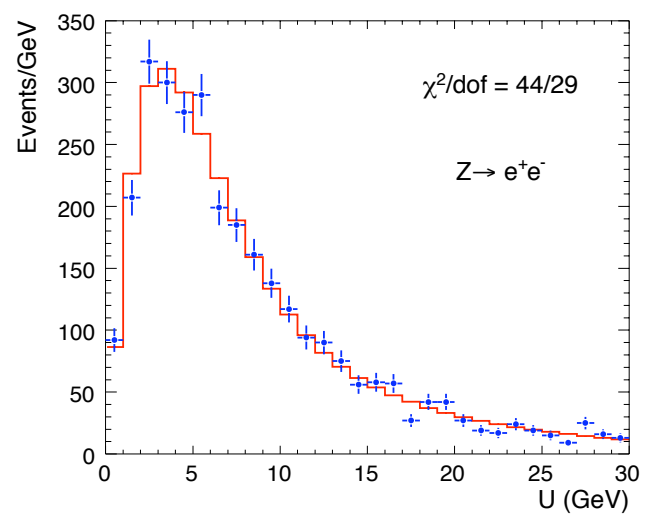

(e)

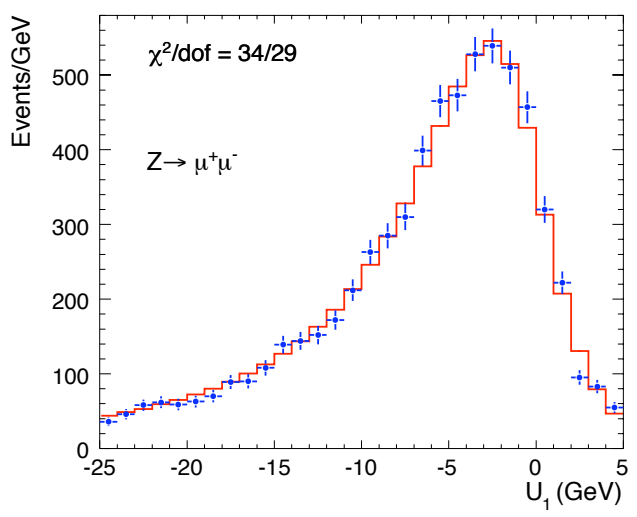

(b)

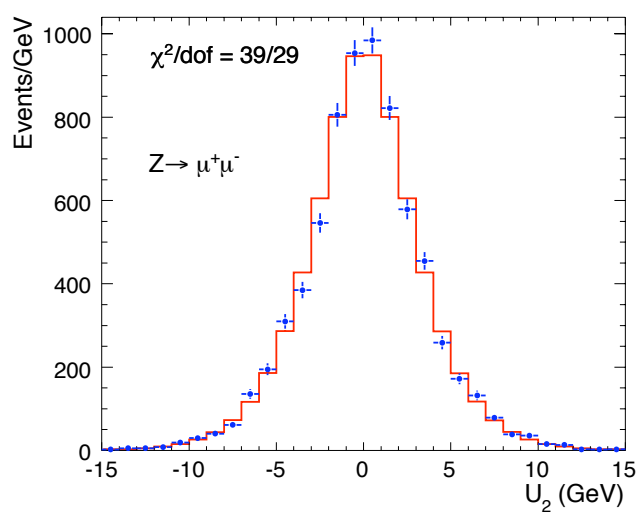

(d)

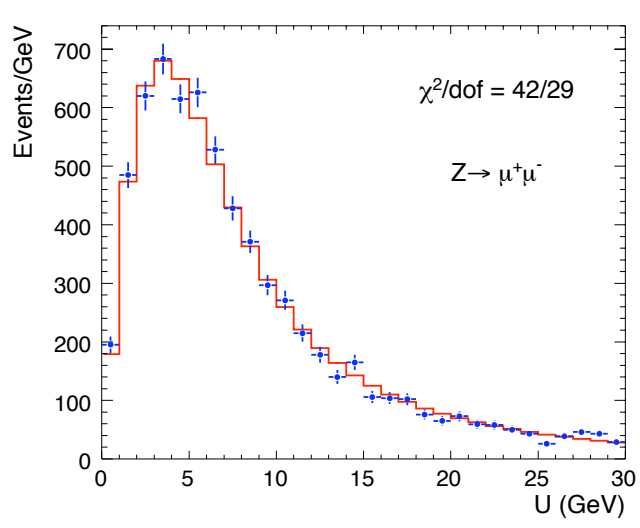

(f)

Figure 7.20: Recoil distributions of (a) and (b) $U_{1}$, (c) and (d) $U_{2}$ and (e) and (f) $U$ for the Z $\rightarrow e^{+} e^{-}$ and $\mathrm{Z} \rightarrow \mu^{+} \mu^{-}$channels compared to the best fit simulation in the $\mathrm{W}$ width analysis. 


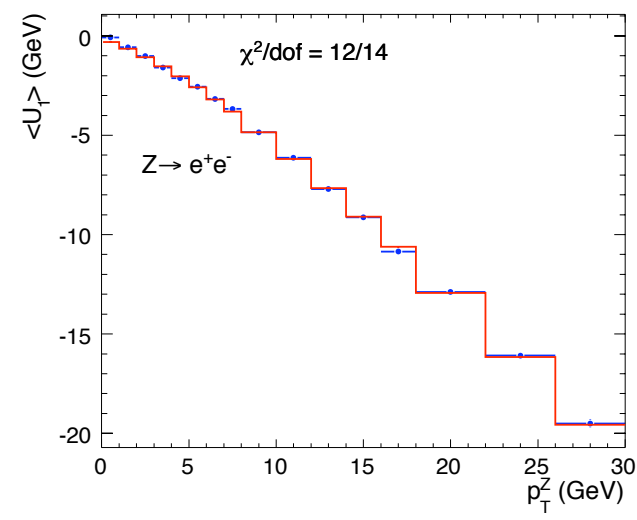

(a)

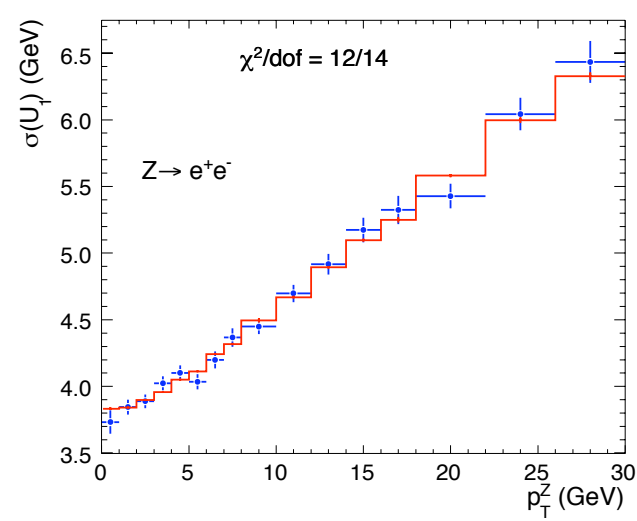

(c)

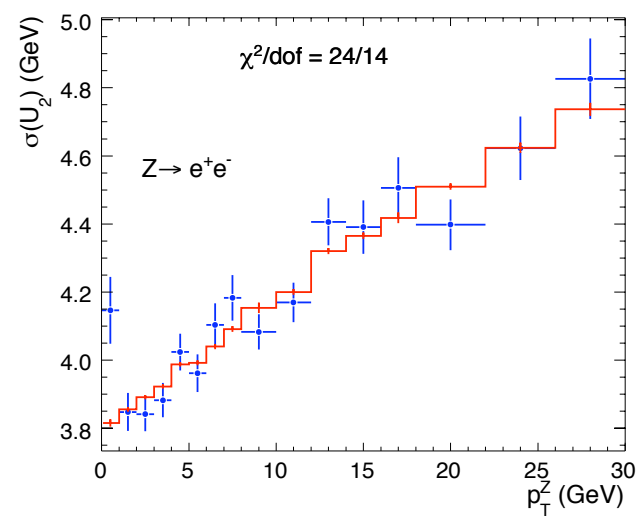

(e)

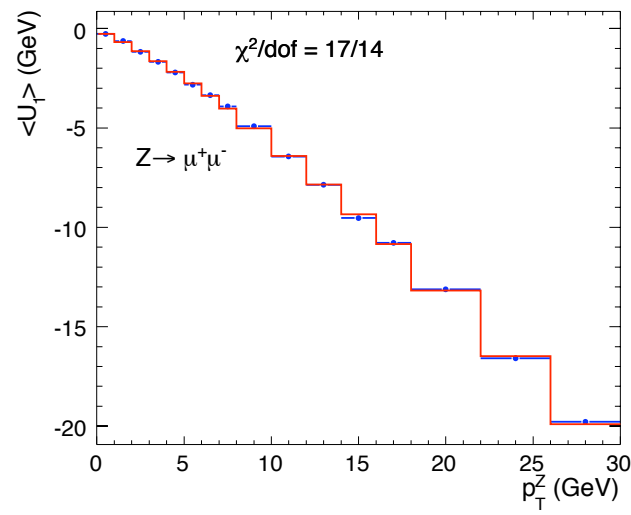

(b)

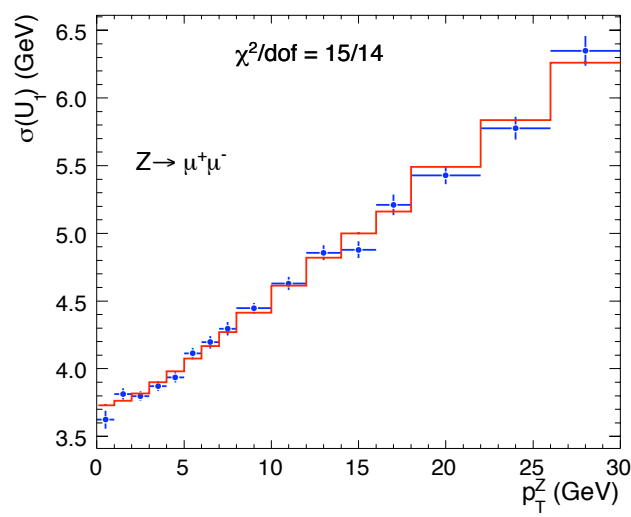

(d)

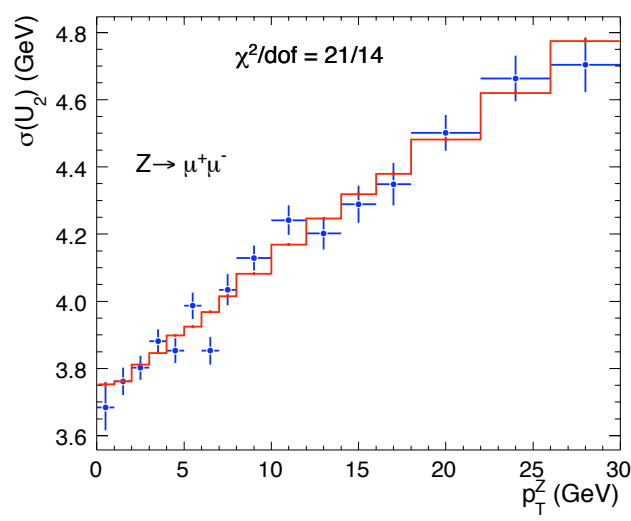

(f)

Figure 7.21: The distributions of (a) and (b) $\left\langle U_{1}\right\rangle$ vs. $p_{T}^{Z},(\mathrm{c})$ and (d) $\sigma\left(U_{1}\right)$ vs. $p_{T}^{Z}$, (e) and (f) $\sigma\left(U_{2}\right)$ vs. $p_{T}^{Z}$ in $\mathrm{Z} \rightarrow e^{+} e^{-}$and $\mathrm{Z} \rightarrow \mu^{+} \mu^{-}$events compared to the best fit simulation in the $\mathrm{W}$ mass analysis. 


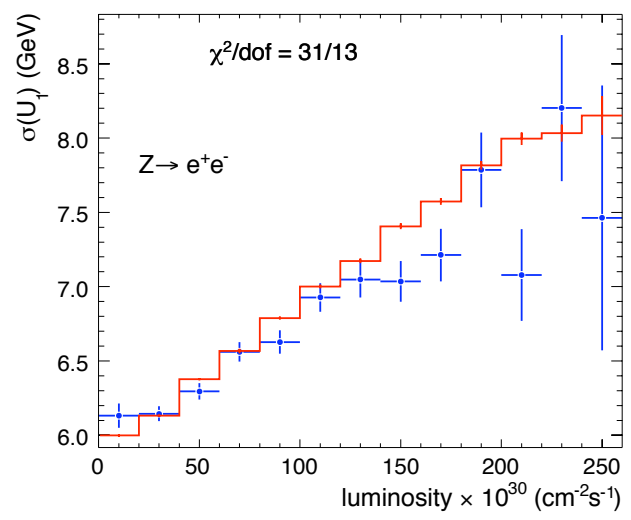

(a)

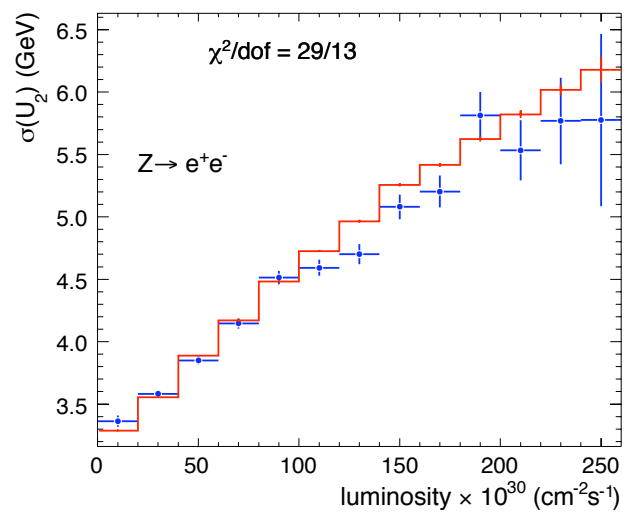

(c)

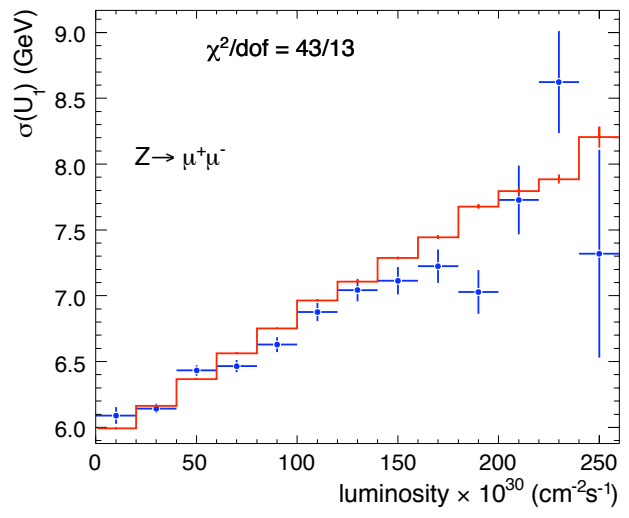

(b)

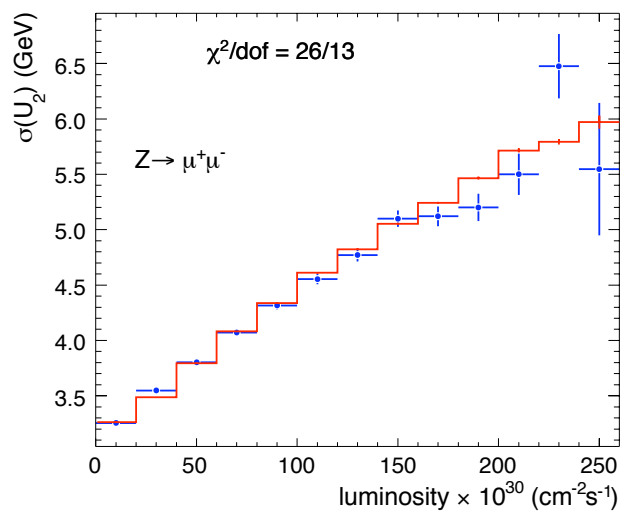

(d)

Figure 7.22: Distributions showing the dependence of the $U_{1}$ and $U_{2}$ resolutions on luminosity in the data compared to the simulation using the best fit recoil parameters in the $\mathrm{W}$ mass analysis.

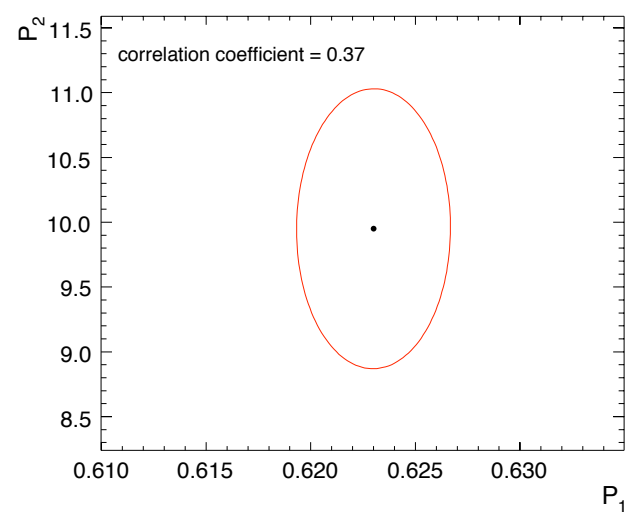

Figure 7.23: Constant $\chi^{2}$ contour of the $\left\langle U_{1}\right\rangle$ vs $p_{T}^{Z}$ distribution in $\mathrm{Z} \rightarrow e^{+} e^{-}$events as the two response parameters $P_{1}$ and $P_{2}$ are varied in the $\mathrm{W}$ mass analysis. 


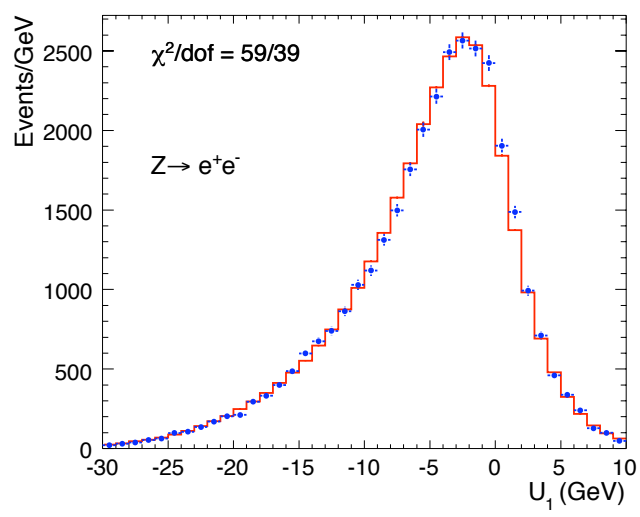

(a)

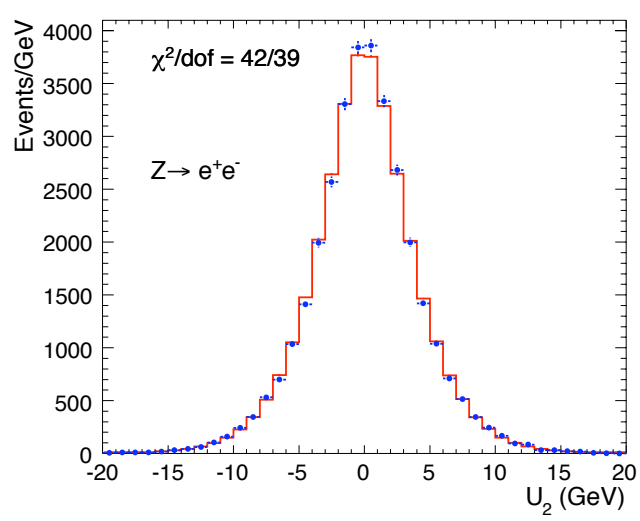

(c)

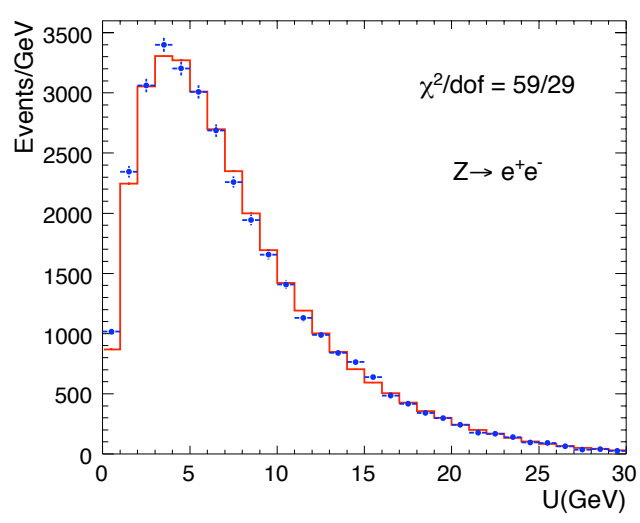

(e)

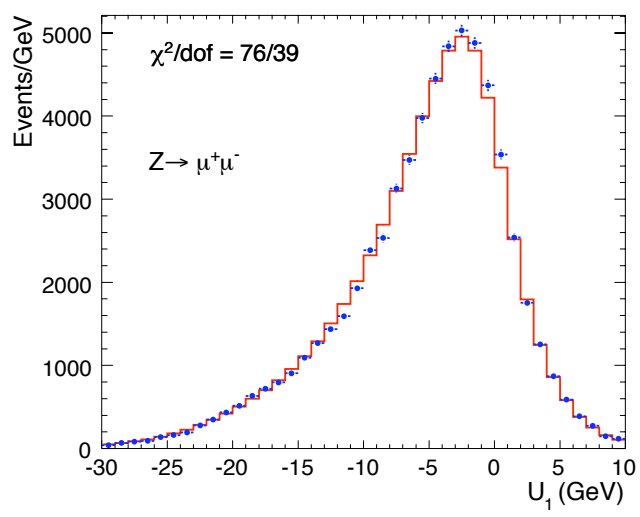

(b)

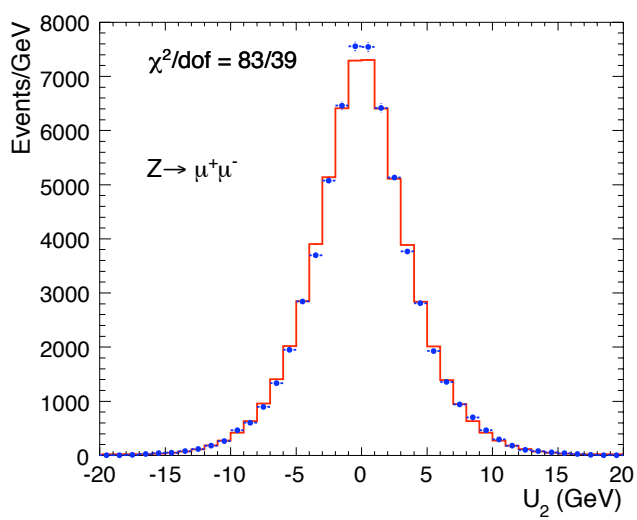

(d)

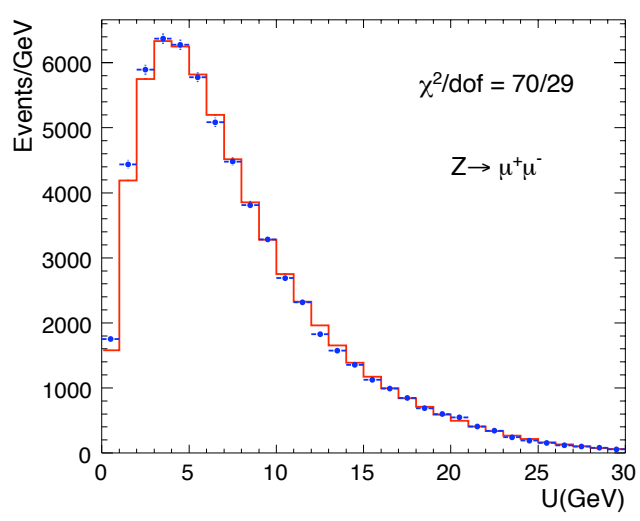

(f)

Figure 7.24: Recoil distributions of $U, U_{1}$ and $U_{2}$ for the $\mathrm{Z} \rightarrow e^{+} e^{-}$and $\mathrm{Z} \rightarrow \mu^{+} \mu^{-}$channels compared to the best fit simulation in the $\mathrm{W}$ mass analysis. 


\subsection{Recoil Comparison}

The recoil model described in the previous section is used to simulate the recoil in $\mathrm{W} \rightarrow l \nu$ events using the best fit recoil parameters obtained in the fits to $\mathrm{Z} \rightarrow l^{+} l^{-}$data as given in Table 7.4 for the $\mathrm{W}$ width analysis and Table 7.5 for the $\mathrm{W}$ mass analysis. The true $\mathrm{W} p_{\mathrm{T}}$ and the luminosity as sampled from $\mathrm{W} \rightarrow l \nu$ data are used to construct $U_{1}$ and $U_{2}$ which are subsequently rotated to give $U_{x}$ and $U_{y}$. Since the $p_{\mathrm{T}}$ of the $\mathrm{W}$ boson is not known in the data, the distributions of $U_{1}$ and $U_{2}$ cannot be compared for the $\mathrm{W}$ samples. Instead, the direction of the charged lepton is used to project the recoil vector parallel to the lepton $\left(U_{\|}\right)$and perpendicular to the lepton $\left(U_{\perp}\right)$. Figure 7.25 shows the distributions of $U, U_{\|}$ and $U_{\perp}$ for $\mathrm{W} \rightarrow e \nu$ and $\mathrm{W} \rightarrow \mu \nu$ events in the $\mathrm{W}$ width measurement. In general there is satisfactory agreement between data and simulation. The mean and standard deviations of the distributions are also shown, the most important of these quantities for the transverse mass is the mean of $U_{\|}$. This is consistent within errors between data and simulation for both the electron and muon channels. The $\not_{T}$ distribution is given in Figure 7.26 for the electron and muon channels in the $\mathrm{W}$ width measurement. It shows that the distribution is well described by the simulation. The variation of $\left\langle U_{\|}\right\rangle$with $M_{T}, U$ and $\Delta \phi(U, l)$ is shown in Figures 7.27 and 7.28. The variation of the resolution of $U_{\perp}$ with $U$ is shown in Figure 7.28. The distributions show that the correlations between recoil quantities is well described by the simulation.

The distributions of $U, U_{\|}$and $U_{\perp}$ are shown for the $\mathrm{W}$ mass analysis in the electron and muon channels in Figure 7.29. The dependence of $\left\langle U_{\|}\right\rangle$on $M_{T}, U$ and $\Delta \phi(U, l)$ is shown in Figures 7.30 and 7.31 and the resolution of $U_{\perp}$ as a function of $U$ is shown in Figure 7.31. Figure 7.29 shows that the mean of $U_{\|}$is consistent within approximately $2.5 \sigma$ between data and simulation for the electron channel and approximately $1.5 \sigma$ for the muon channel. However, it also shows that $U$ is not well described in the simulation, particularly in the low $U$ region. The effect of this discrepancy on the $M_{T}$ distribution 
can be estimated by applying a weight to each event in the simulation such that the $U$ distribution in the simulation perfectly describes that in the data. The event weights applied in the simulation are taken from the ratio of the $U$ distribution in the data to the simulation. The $M_{T}$ distribution obtained after reweighting the simulation events is fitted using simulation templates of the $M_{T}$ distribution with no reweighting applied. A shift of $5 \mathrm{MeV}(7 \mathrm{MeV})$ in the mass of the $\mathrm{W}$ boson in obtained in $\mathrm{W} \rightarrow e \nu(\mathrm{W} \rightarrow \mu \nu)$ events using this method.

A discrepancy was also noticed in the description of $U$ in the low $\Sigma E_{\mathrm{T}}$ region, shown in Figure 7.32. It is evident from Figure 7.32 that the RMS of $U$ in the simulation for $\Sigma E_{\mathrm{T}}<20 \mathrm{GeV}$ is much larger than that in the data. The effect of this discrepancy on the fitted mass of the $\mathrm{W}$ boson is evaluated in a similar way as the discrepancy in the inclusive $U$ distribution. A histogram of weights is obtained by taking the ratio of the $U$ distribution in the data to simulation in the region $\Sigma E_{\mathrm{T}}<20 \mathrm{GeV}$. The $M_{T}$ distribution obtained after this reweighting is fitted using simulation templates of the $M_{T}$ distribution with no such weights applied. A shift of $7 \mathrm{MeV}(10 \mathrm{MeV})$ in the value of the fitted mass is obtained for the $\mathrm{W} \rightarrow e \nu(\mathrm{W} \rightarrow \mu \nu)$ channel. A comparison of the $E_{T}$ distribution in data and simulation is shown in Figure 7.33 for $\mathrm{W} \rightarrow e \nu$ and $\mathrm{W} \rightarrow \mu \nu$ events. It shows that $\not_{T}$ is reasonably well described in the simulation.

\subsection{Recoil Systematic}

A $7 \times 7$ covariance matrix is constructed using the 7 parameters describing the recoil model in the $\mathrm{W}$ width analysis. The uncertainties on the parameters given in Table 7.4 are obtained from the diagonal elements of the covariance matrix. The systematic uncertainty on $\Gamma_{W}$ is evaluated by sampling from the covariance matrix 250 times to obtain 250 sets of recoil parameters that are within the statistical uncertainty of the $\mathrm{Z} \rightarrow l^{+} l^{-}$recoil fits. This effectively provides 250 recoil models each of which is used to produce a $M_{T}$ 
distribution. Simulation templates of the $M_{T}$ distribution with different input W width values and the default set of recoil parameters are produced and each of the $250 M_{T}$ distributions are fit using the templates to obtain the best fit value for $\Gamma_{W}$. The 250 fit values for $\Gamma_{W}$ are plotted and the RMS of a Gaussian fit to the distribution is taken as the uncertainty on $\Gamma_{W}$ from the recoil. The systematic uncertainty obtained for the electron and muon channels is given in Table 7.6. The systematic uncertainty from the response of $U_{1}$ and the resolutions can also be obtained separately by assuming that the $U_{1}$ response and resolution parameters are completely uncorrelated and splitting up the $7 \times 7$ covariance matrix into a $3 \times 3$ matrix for the response and two $2 \times 2$ covariance matrices for the $U_{1}$ and $U_{2}$ resolutions. The systematic uncertainty obtained from the separate response and resolutions is also given in Table 7.6.

In addition to fitting to the $M_{T}$ distribution to obtain the systematic for the $\mathrm{W}$ width, the lepton $p_{\mathrm{T}}$ distribution can also be fit to obtain the $\mathrm{W}$ width. The systematic uncertainty on $\Gamma_{W}$ when fitting to the lepton $p_{\mathrm{T}}$ can be obtained in the same way as that explained above for the transverse mass. The recoil systematic is found to be $74 \mathrm{MeV}$ for $\mathrm{W} \rightarrow e \nu$ events and $69 \mathrm{MeV}$ for $\mathrm{W} \rightarrow \mu \nu$ events for lepton $p_{\mathrm{T}}>45 \mathrm{GeV}$.

The recoil parameters for the electron and muon channels given in Table 7.4 are consistent with each other within $1.5 \sigma$ if correlations are ignored. However, there are correlations between the parameters. The level of compatibility between the electron and muon recoil parameters can be evaluated using the covariance matrices. The electron covariance matrix is sampled 150 times with each sampling producing a set of $\mathrm{Z} \rightarrow e^{+} e^{-}$and $\mathrm{W} \rightarrow e \nu$ recoil distributions using the sampled set of recoil parameters. The spread of the total $\chi^{2}$ of the three $\mathrm{Z}$ distributions used in the recoil fit and the total $\chi^{2}$ of three $\mathrm{W}$ distributions $\left(U, U_{\|}, U_{\perp}\right)$ is shown by the black points in Figure 7.34(a). The muon covariance matrix is then sampled 150 times for $\mathrm{Z} \rightarrow e^{+} e^{-}$and $\mathrm{W} \rightarrow e \nu$ events and the spread of the total $\chi^{2}$ of $\mathrm{Z}$ distributions and that of the $\mathrm{W}$ distributions is also shown in Figure 7.34(a). The 
degree of overlap of the two scatter plots can be used to evaluate the level of compatibility between the electron and muon recoil parameters. There are approximately $44 \%$ of points obtained from sampling the muon covariance matrix that are within $1 \sigma$ of the best fit electron point and $87 \%$ are within $2 \sigma$. In addition, using the muon recoil parameters for $\mathrm{Z} \rightarrow e^{+} e^{-}$events increases the total $\chi^{2}$ of the three fitted $\mathrm{Z}$ distributions from $26 / 31$ to $33 / 31$.

The same can be done for the muon channel. Figure 7.34(b) shows the results of sampling the muon covariance matrix and then the electron covariance matrix for $\mathrm{Z} \rightarrow \mu^{+} \mu^{-}$ and $\mathrm{W} \rightarrow \mu \nu$ events. The electron parameters are less well constrained due to lower statistics and there is a smaller degree of overlap. Using the electron recoil parameters for $\mathrm{Z} \rightarrow \mu^{+} \mu^{-}$events increases the total $\chi^{2}$ of the three fitted $\mathrm{Z}$ distributions from $29 / 31$ to $44 / 31$.

It can also be noted from the scatter plots in Figure 7.34 that it is possible to get a large range of $\chi^{2}$ for the $\mathrm{W}$ distributions whilst maintaining a reasonable description of the $\mathrm{Z}$ distributions. In particular, it is found that the $\chi^{2}$ of the $U$ distribution in $\mathrm{W} \rightarrow e \nu$ events can be reduced by choosing a set of recoil parameters from the sampling that minimise the $\chi^{2}$ of the $U$ distribution whilst maintaining a good description of the recoil in $\mathrm{Z} \rightarrow e^{+} e^{-}$events. This set of recoil parameters are referred to as the ' $U$ optimised' parameters. Figures 7.35 and 7.36 show the changes in $\chi^{2}$ for some of the recoil distributions in $\mathrm{Z} \rightarrow e^{+} e^{-}$and $\mathrm{W} \rightarrow e \nu$ events when using the best fit recoil parameters and the ' $U$ optimised' parameters. The plots show that a different set of parameters can considerably improve the description of the recoil in $\mathrm{W}$ data without much degradation in the $\mathrm{Z}$ distributions.

Furthermore, all of the $\chi^{2}$ values on the distributions reflect the statistical uncertainty only. However, there are also systematic uncertainties with contributions from the uncertainty on the boson $p_{\mathrm{T}}$ distribution, backgrounds and the recoil parameter determination 
which is obtained by sampling the covariance matrix of the parameters and estimating the spread of the variations in each histogram bin. The $\chi^{2} /$ dof of the recoil distributions would improve if these systematic uncertainties were propagated through. For instance, the $\chi^{2} /$ dof of the $U$ distribution in $\mathrm{W} \rightarrow e \nu$ events reduces from 86/40 to 56/40 and that in $\mathrm{W} \rightarrow \mu \nu$ events goes from $52 / 40$ to $37 / 40$ when statistical and systematic uncertainties are taken into account. The $U$ distribution and the uncertainty on the shape of the distribution due to the uncertainty on the recoil parameter determination, boson $p_{\mathrm{T}}$ and backgrounds is shown in Figure 7.37 for $\mathrm{W} \rightarrow e \nu$ and $\mathrm{W} \rightarrow \mu \nu$ events. It is evident that the dominant systematic uncertainty on the shape of the $U$ distribution is from the recoil parameter determination uncertainty whilst the uncertainty on the shape due to backgrounds in negligible.

In the $\mathrm{W}$ mass analysis, the recoil model is parameterised using 6 parameters. In the same way as the $\mathrm{W}$ width analysis, a $6 \times 6$ covariance matrix is constructed and the systematic uncertainty on $M_{W}$ is determined by sampling from this covariance matrix 100 times to obtain $100 M_{T}$ distributions with each corresponding to a set of recoil parameters that are within the statistical uncertainty of the $\mathrm{Z} \rightarrow l^{+} l^{-}$recoil fits. Each of these $M_{T}$ distributions are fit using simulation templates of the $M_{T}$ distribution with different input $M_{W}$ values. The spread of the $M_{W}$ values obtained from these fits is shown in Figure 7.38. The RMS of a Gaussian fit to the distribution is used to obtain the systematic uncertainty on $M_{W}$. This is shown in Table 7.7 for the electron and muon channels and the systematic uncertainty obtained from sampling separately from the response and resolution covariance matrices is also given. Table 7.8 shows a breakdown of the systematics from the mismodelling in the $U$ distribution, the discrepancy in the low $\Sigma E_{\mathrm{T}}$ region, the discrepancy in the $\sigma\left(U_{2}\right)$ vs. $p_{\mathrm{T}}$ plot and from tuning only on $\mathrm{CC} \mathrm{Z}$ events, where it has been assumed that the $6 \mathrm{MeV}$ systematic from only considering $\mathrm{CC}$ events is the same for the muon channel. 
Background contamination in the $\mathrm{Z}$ event samples can affect the recoil parameters obtained in the fits to the $\mathrm{Z}$ distributons. Chapter 9 describes the methods used to evaluate the normalisation and shape of the various backgrounds contributing to the $\mathrm{Z} \rightarrow e^{+} e^{-}$ event sample. Of these, the shape of the QCD background is the most dominating for the recoil distributions. The effect of this background on the $M_{T}$ distribution in $\mathrm{W}$ events is evaluated by fitting for the recoil parameters when the QCD background in $\mathrm{Z}$ events is varied by its error, in this case the maximum QCD background value of $0.43 \%$ is chosen. Simulation templates of the $M_{T}$ distribution with varying input $\mathrm{W}$ mass values are fitted to the $M_{T}$ distribution obtained using these recoil parameters and the shift in the value of the $\mathrm{W}$ mass is found to be negligible.

Background contamination in the $\mathrm{W}$ samples can also affect the recoil distributions, with $U_{\|}$being the most important since a bias in this distribution produces a direct bias in the $M_{T}$ distribution. The $U_{\|}$distribution for the backgrounds contributing to the electron channel in the W mass analysis is shown in Figure 7.39. It is evident that the shape of the $U_{\|}$distribution in the $\mathrm{Z} \rightarrow e^{+} e^{-}$and QCD backgrounds is noticably skewed towards negative $U_{\|}$values and can produce a bias in the mean of $U_{\|}$in the data. The backgrounds shown in Figure 7.39 produce an overall shift of $7 \mathrm{MeV}$ in the mean of $U_{\|}$. Chapter 9 discusses the methods used to estimate the amount and shape of backgrounds contributing to the electron channel. They are added to the simulation histograms and included in all subsequent recoil plots.

It was shown in the previous $\mathrm{W}$ mass analysis [22] that the dominant backgrounds contributing to the muon channel were the two electroweak backgrounds; $\mathrm{Z} \rightarrow \mu^{+} \mu^{-}$and $\mathrm{W} \rightarrow \tau \nu$. For the muon channel therefore, only these backgrounds are included in the recoil plots. The normalisation and shape of the backgrounds is obtained from CdfSim event samples using a method analogous to that described in Section 9.1.1. The $\mathrm{Z} \rightarrow \mu^{+} \mu^{-}$ background is found to be $8.6 \%$ of the $\mathrm{W} \rightarrow \mu \nu$ sample and the $\mathrm{W} \rightarrow \tau \nu$ background is found to be $0.88 \%$. 
The systematic uncertainty on the $\mathrm{W}$ mass from the statistical uncertainty on the recoil parameters given in Table 7.7 and the systematic uncertainty from various other contributions given in Table 7.8 can be combined in quadrature to give a final recoil systematic of $14 \mathrm{MeV}$ for the electron channel and $15 \mathrm{MeV}$ for the muon channel. A combined recoil systematic for the electron and muon channels is found to be $13 \mathrm{MeV}$, taking into account all the correlations between the various sources of systematics.

\begin{tabular}{|l|cc|}
\hline & $\mathrm{W} \rightarrow e \nu$ & $\mathrm{W} \rightarrow \mu \nu$ \\
\hline \hline$\Delta \Gamma_{W}$ & 54 & 49 \\
\hline$\Delta \Gamma_{W}($ response $)$ & 44 & 33 \\
$\Delta \Gamma_{W}\left(U_{1}\right.$ resolution $)$ & 37 & 27 \\
$\Delta \Gamma_{W}\left(U_{2}\right.$ resolution $)$ & 10 & 6 \\
\hline
\end{tabular}

Table 7.6: Systematic uncertainty on $\Gamma_{W}$ from the recoil in the electron and muon channels for the W width analysis. $\Delta \Gamma_{W}$ (response) gives the systematic on $\Gamma_{W}$ from the three response parameters, $\Delta \Gamma_{W}\left(U_{1}\right.$ resolution) and $\Delta \Gamma_{W}\left(U_{2}\right.$ resolution) give the systematic from the resolution parameters.

\begin{tabular}{|l|cc|}
\hline & $\mathrm{W} \rightarrow e \nu$ & $\mathrm{W} \rightarrow \mu \nu$ \\
\hline \hline$\Delta M_{W}$ & 8 & 5 \\
\hline$\Delta M_{W}($ response $)$ & 5 & 3 \\
$\Delta M_{W}\left(U_{1}\right.$ resolution $)$ & 4 & 2 \\
$\Delta M_{W}\left(U_{2}\right.$ resolution $)$ & 5 & 3 \\
\hline
\end{tabular}

Table 7.7: Systematic uncertainty on $M_{W}$ from the recoil in the electron and muon channels for the W mass analysis. $\Delta M_{W}$ (response) gives the systematic on $M_{W}$ from the three response parameters, $\Delta M_{W}\left(U_{1}\right.$ resolution) and $\Delta M_{W}\left(U_{2}\right.$ resolution $)$ give the systematic from the resolution parameters.

\begin{tabular}{|l|cc|}
\hline & $\mathrm{W} \rightarrow e \nu$ & $\mathrm{W} \rightarrow \mu \nu$ \\
\hline \hline$U$ discrepancy & 5 & 7 \\
low $\Sigma E_{\mathrm{T}}$ discrepancy & 7 & 10 \\
CC only & 6 & 6 \\
$\sigma\left(U_{2}\right)$ discrepancy & 5 & - \\
luminosity discrepancy & 2.9 & 3.5 \\
\hline
\end{tabular}

Table 7.8: Systematic uncertainty on $M_{W}$ from the discrepancy in the $U$ distribution, the discrepancy in the low $\Sigma E_{\mathrm{T}}$ distribution, the discrepancy in the $\sigma\left(U_{2}\right)$ vs. $p_{\mathrm{T}}$ distribution and tuning the recoil only on $\mathrm{CC}$ events. 


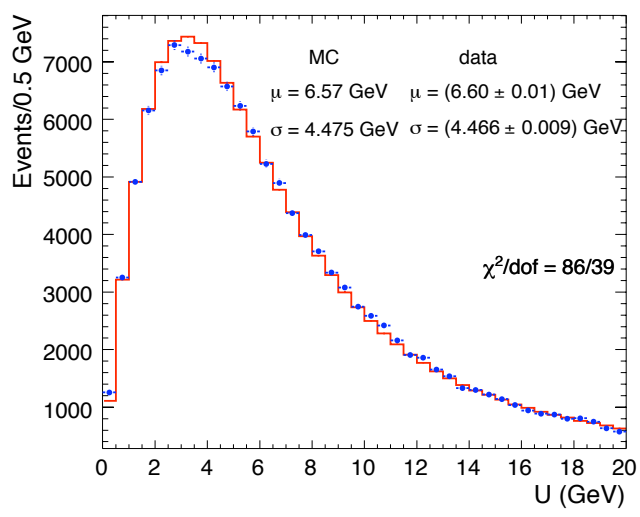

(a)

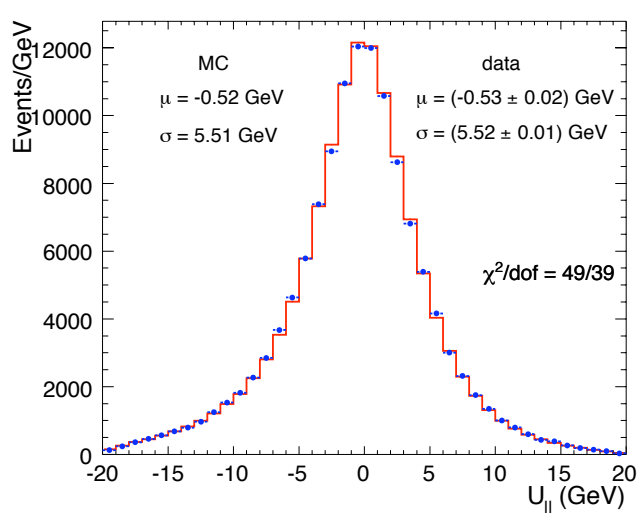

(c)

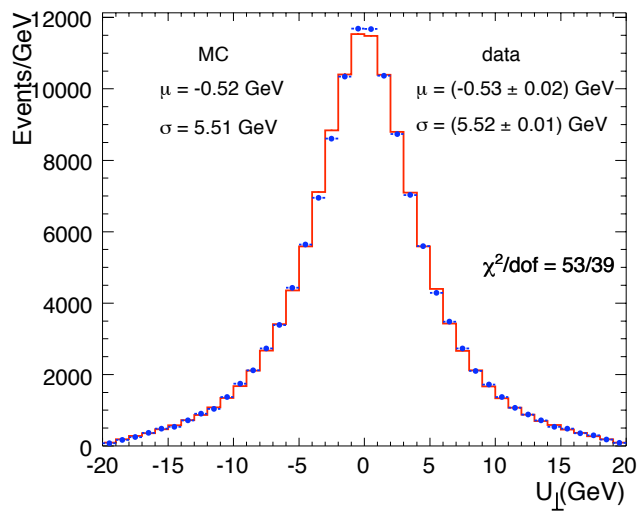

(e)

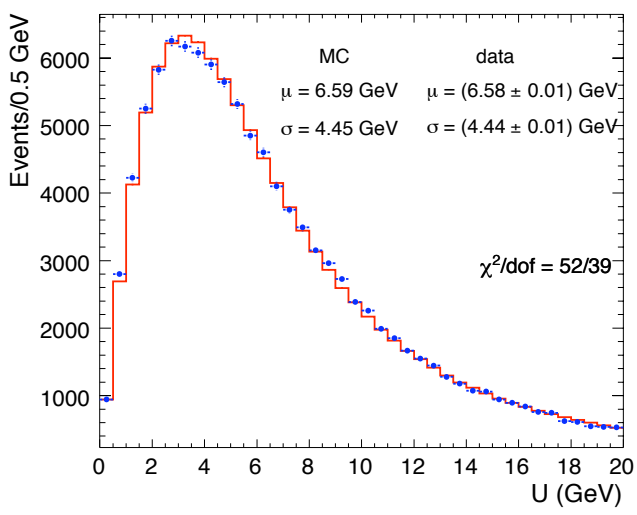

(b)

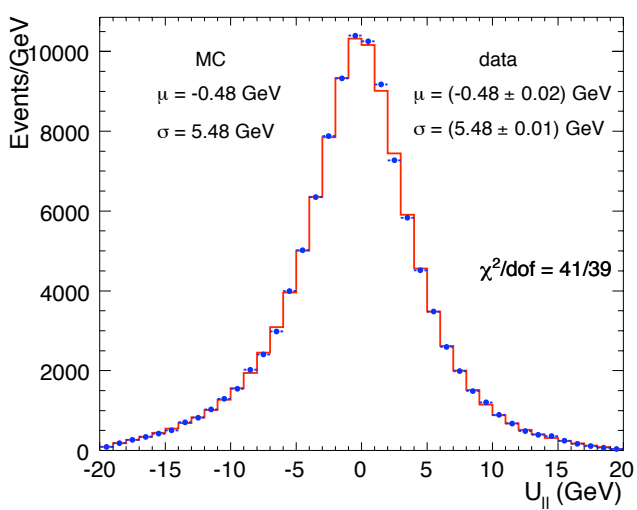

(d)

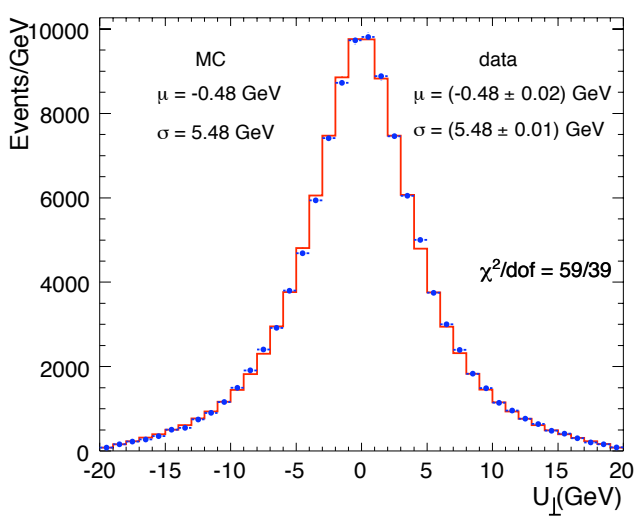

(f)

Figure 7.25: Distributions of $U, U_{\|}$and $U_{\perp}$ in $\mathrm{W} \rightarrow e \nu$ and $\mathrm{W} \rightarrow \mu \nu$ data compared to the simulation for the $\mathrm{W}$ width analysis. 


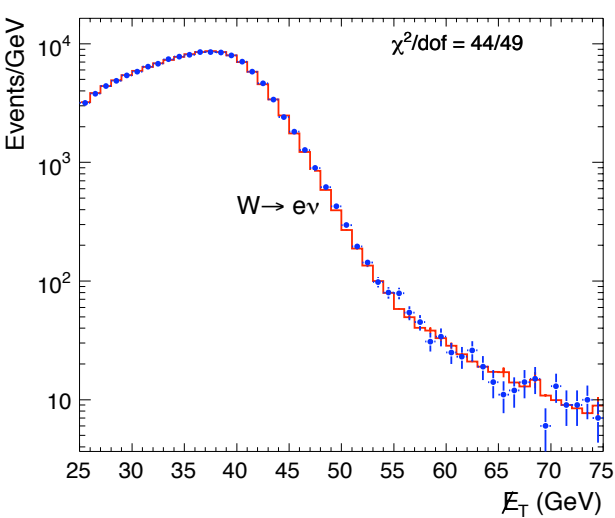

(a)

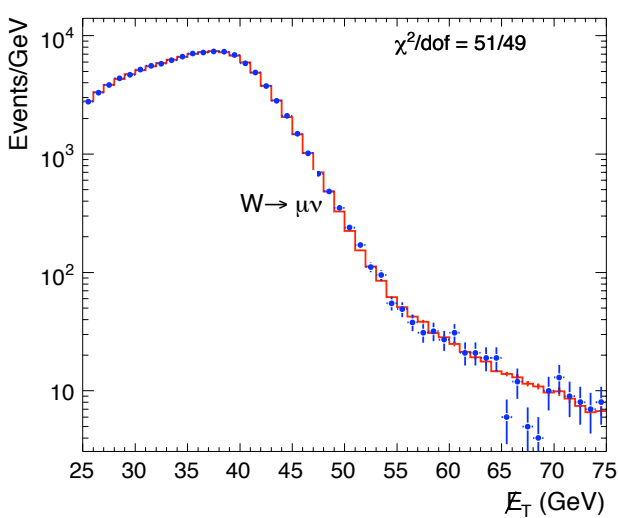

(b)

Figure 7.26: Distribution of $\not_{T}$ in (a) W $\rightarrow e \nu$ and (b) W $\rightarrow \mu \nu$ events compared to the simulation for the $\mathrm{W}$ width analysis.

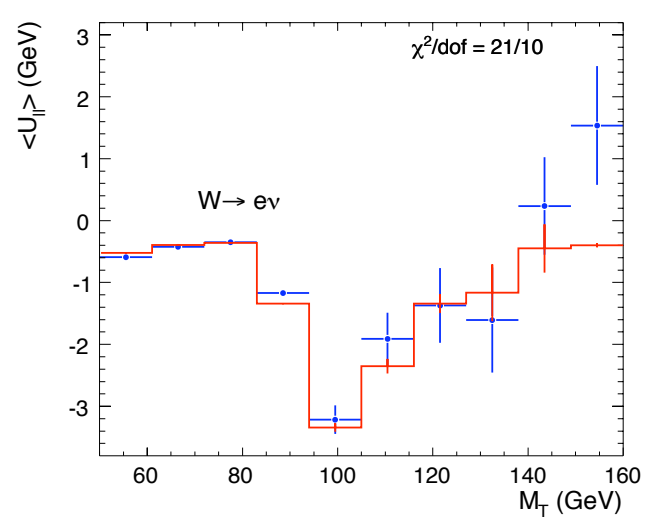

(a)

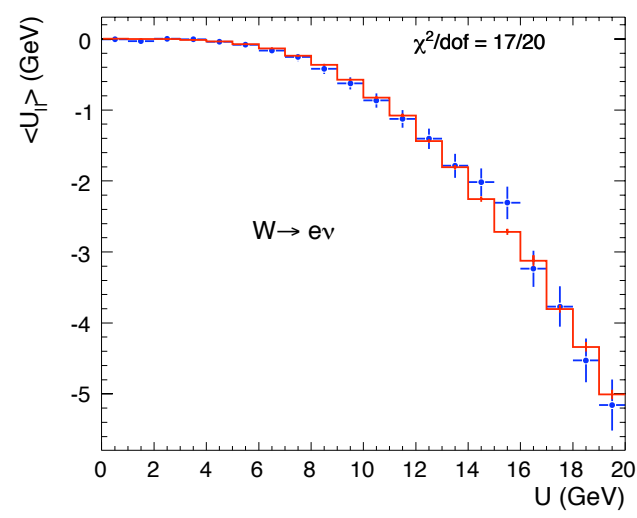

(c)

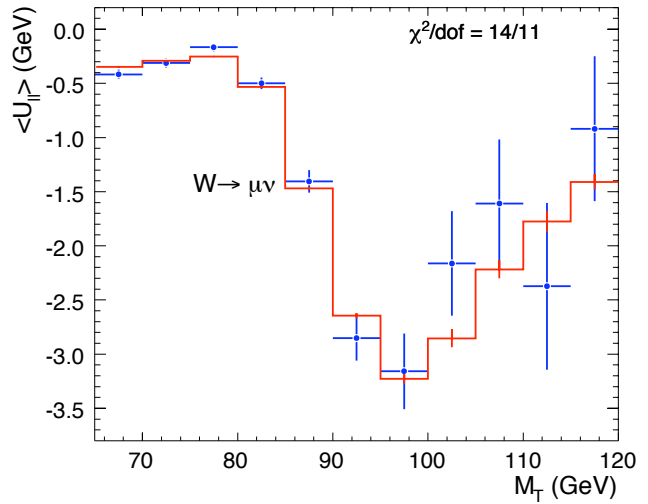

(b)

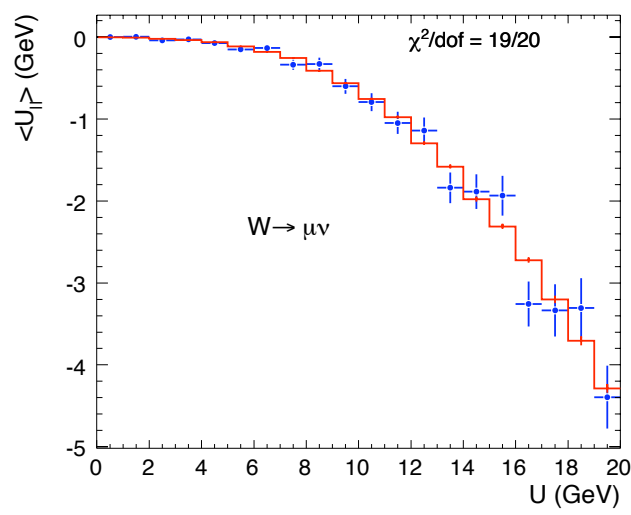

(d)

Figure 7.27: Variation of the $\left\langle U_{\|}\right\rangle$with $M_{T}$ and $U$ in the $\mathrm{W} \rightarrow e \nu$ and $\mathrm{W} \rightarrow \mu \nu$ data compared to the simulation in the $\mathrm{W}$ width analysis. 


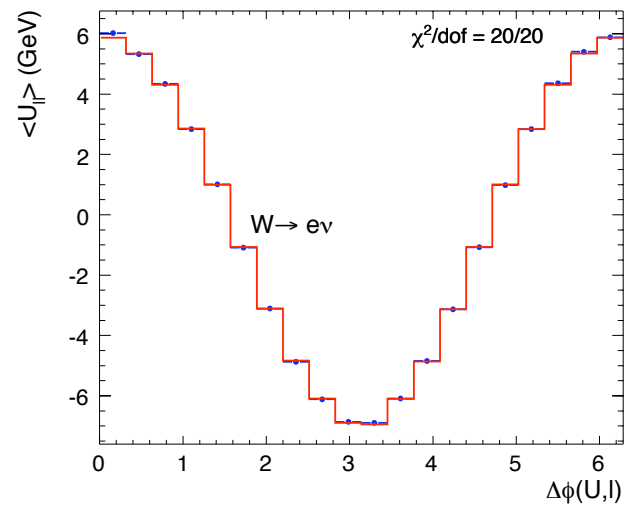

(a)

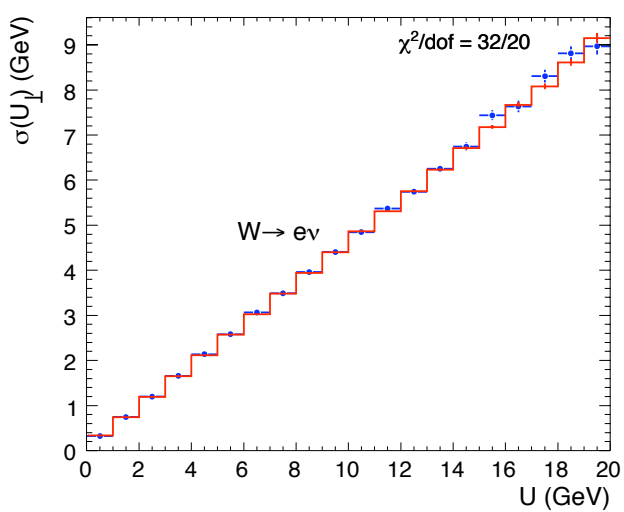

(c)

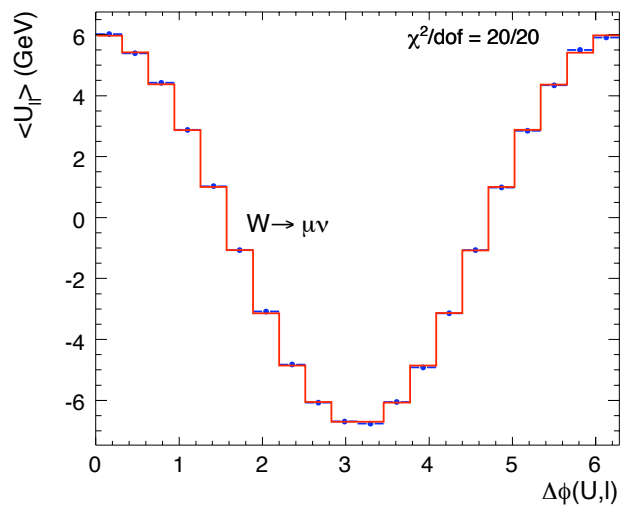

(b)

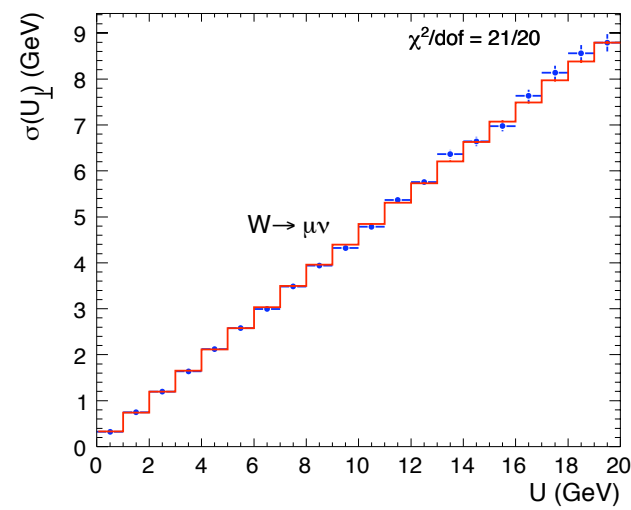

(d)

Figure 7.28: Variation of the $\left\langle U_{\|}\right\rangle$with $\Delta \phi(U, l)$ and $\sigma\left(U_{\perp}\right)$ with $U$ in the $\mathrm{W} \rightarrow e \nu$ and $\mathrm{W} \rightarrow \mu \nu$ data compared to the simulation in the $\mathrm{W}$ width analysis. 


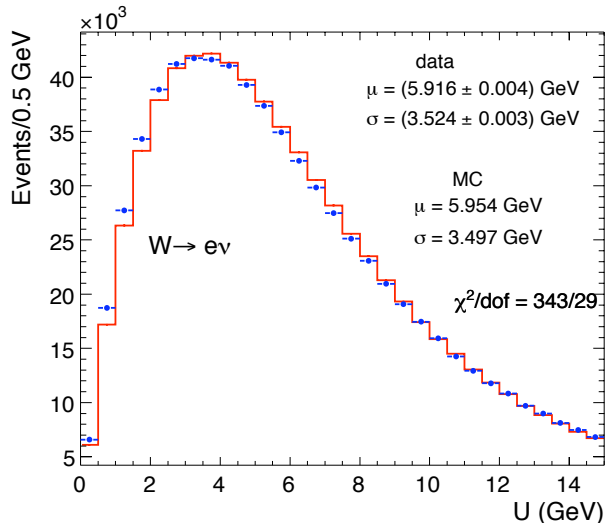

(a)

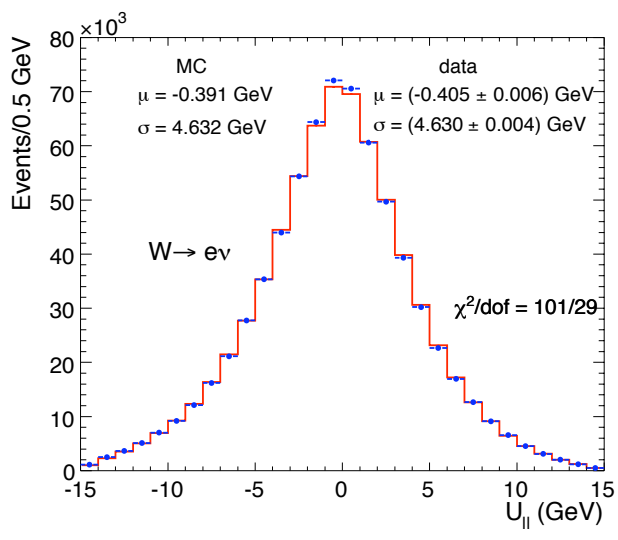

(c)

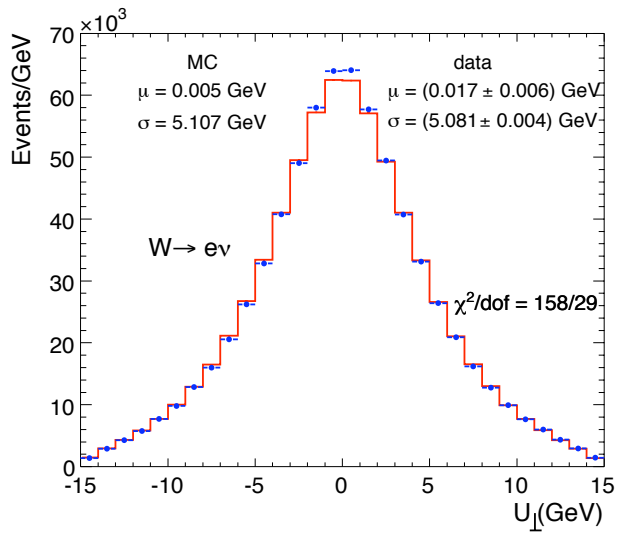

(e)

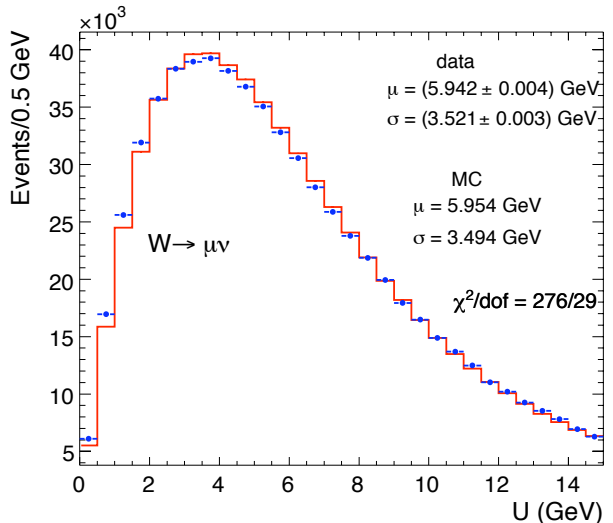

(b)

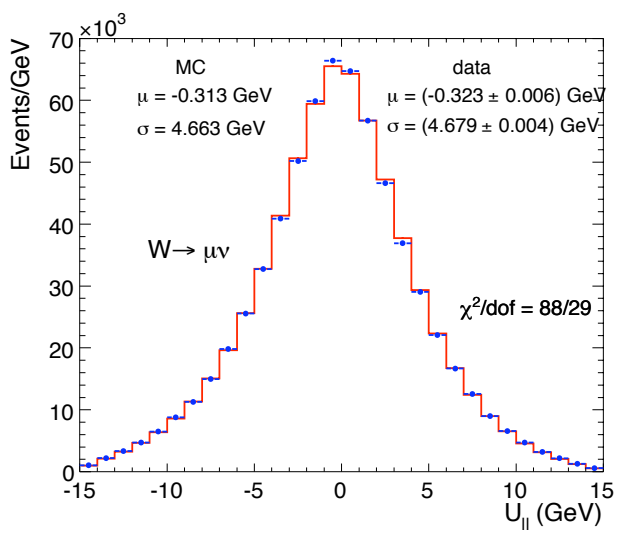

(d)

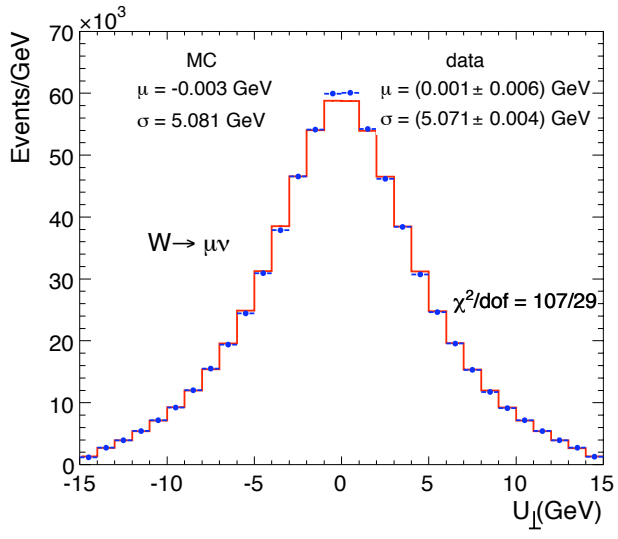

(f)

Figure 7.29: Distributions of $U, U_{\|}$and $U_{\perp}$ in $\mathrm{W} \rightarrow e \nu$ and $\mathrm{W} \rightarrow \mu \nu$ data compared to the simulation for the $\mathrm{W}$ mass analysis. 


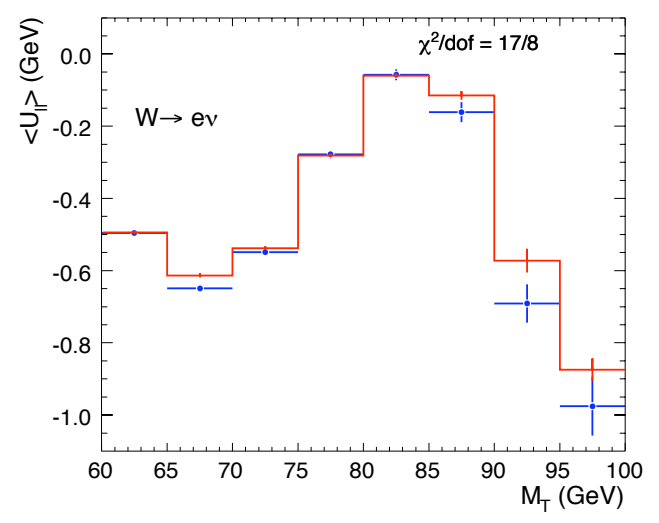

(a)

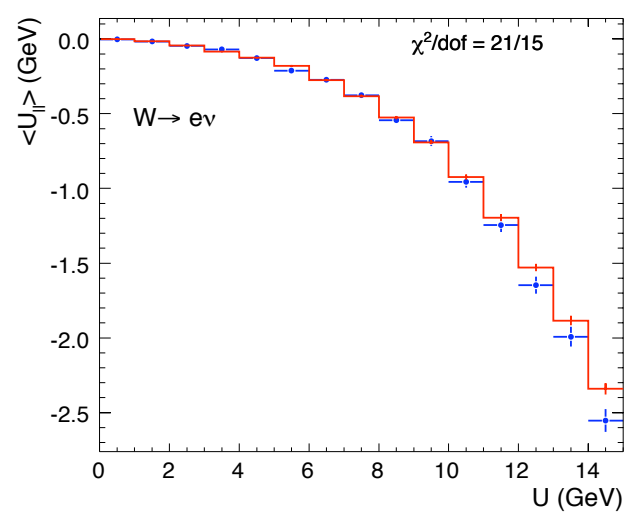

(c)

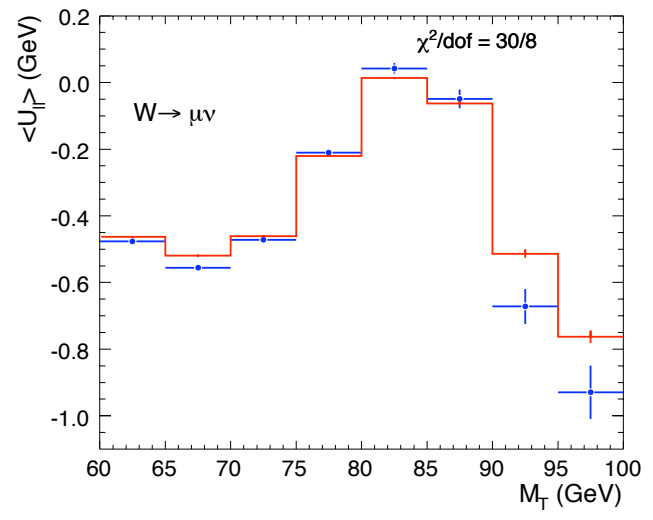

(b)

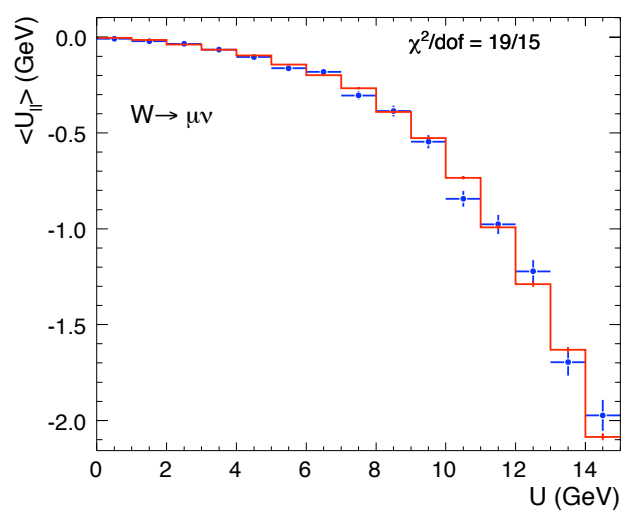

(d)

Figure 7.30: Variation of the $\left\langle U_{\|}\right\rangle$with $M_{T}$ and $U$ in the $\mathrm{W} \rightarrow e \nu$ and $\mathrm{W} \rightarrow \mu \nu$ data compared to the simulation in the $\mathrm{W}$ mass analysis. 


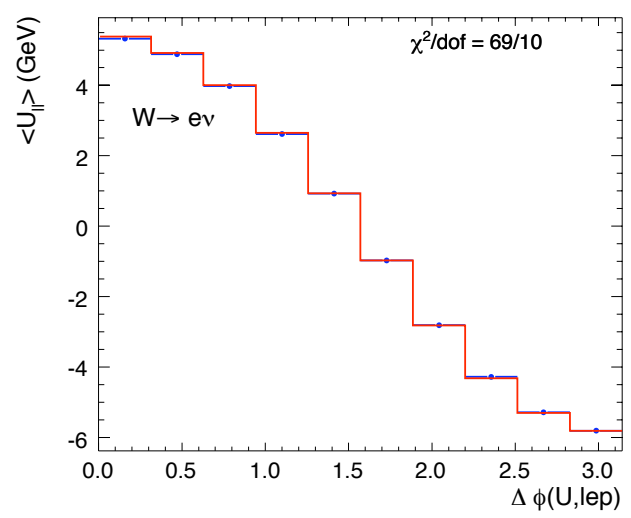

(a)

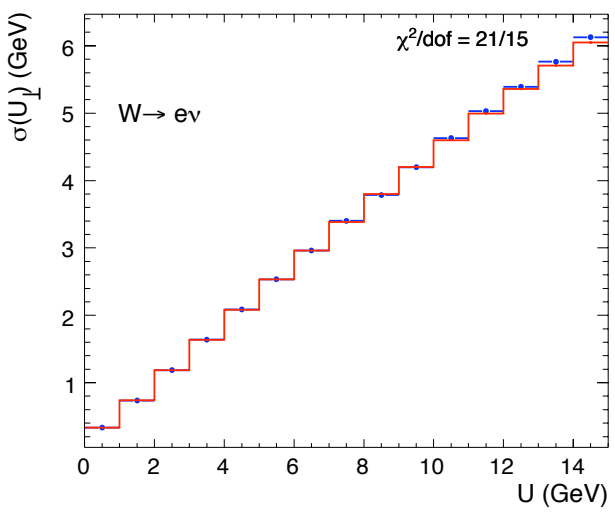

(c)

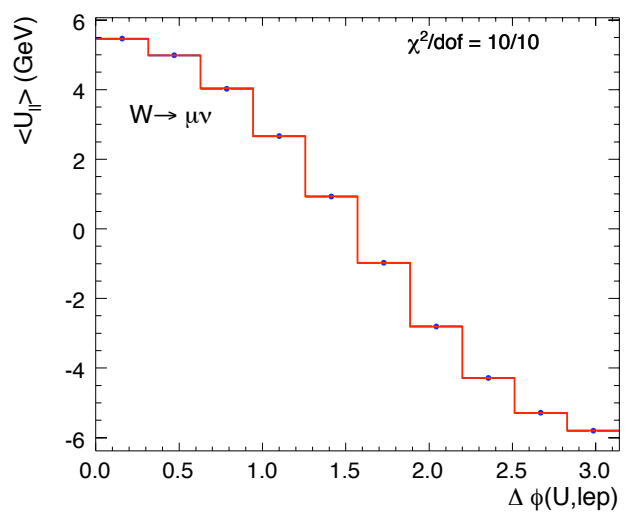

(b)

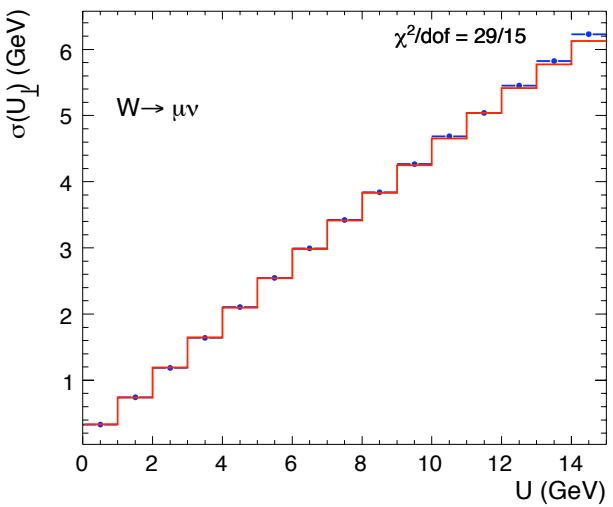

(d)

Figure 7.31: Variation of the $\left\langle U_{\|}\right\rangle$with $\Delta \phi(U, l)$ and $\sigma\left(U_{\perp}\right)$ with $U$ in the $\mathrm{W} \rightarrow e \nu$ and $\mathrm{W} \rightarrow \mu \nu$ data compared to the simulation in the $\mathrm{W}$ mass analysis.

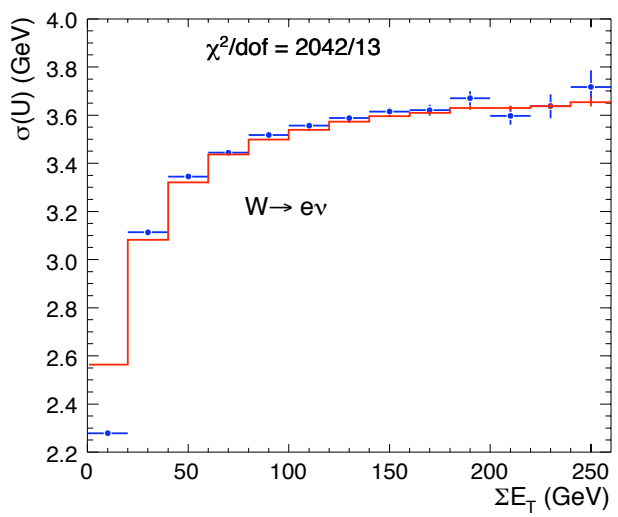

Figure 7.32: Variation of the resolution of $U$ with $\Sigma E_{\mathrm{T}}$ in the $\mathrm{W} \rightarrow e \nu$ data compared to the simulation in the $\mathrm{W}$ mass analysis. 


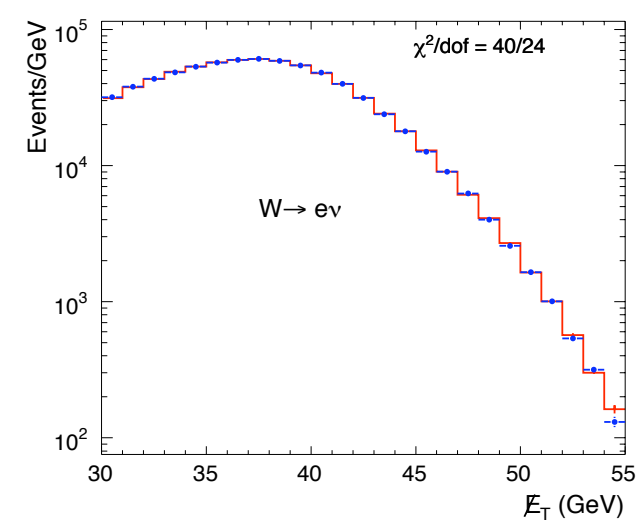

(a)

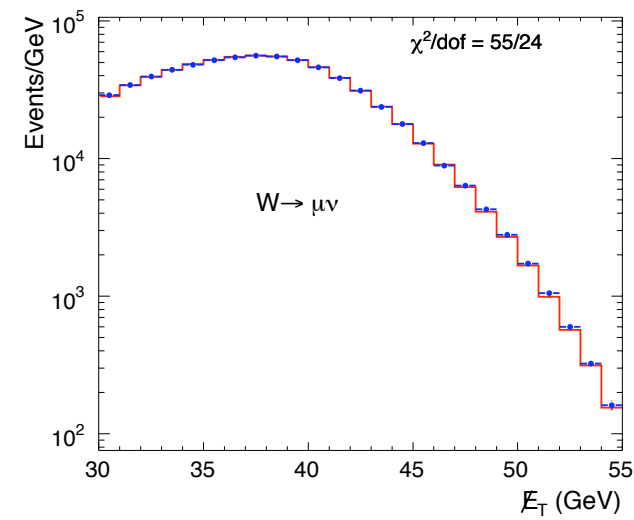

(b)

Figure 7.33: Distribution of $E_{T}$ in (a) $\mathrm{W} \rightarrow e \nu$ and (b) $\mathrm{W} \rightarrow \mu \nu$ events compared to the simulation for the $\mathrm{W}$ mass analysis.

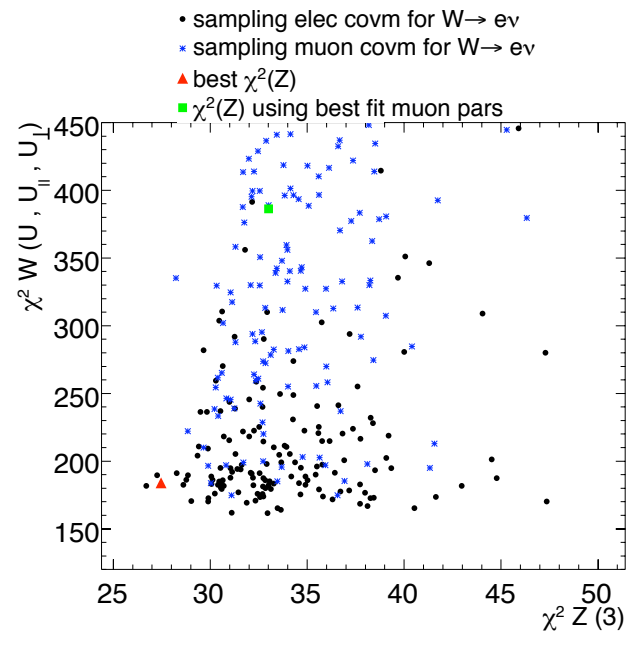

(a)

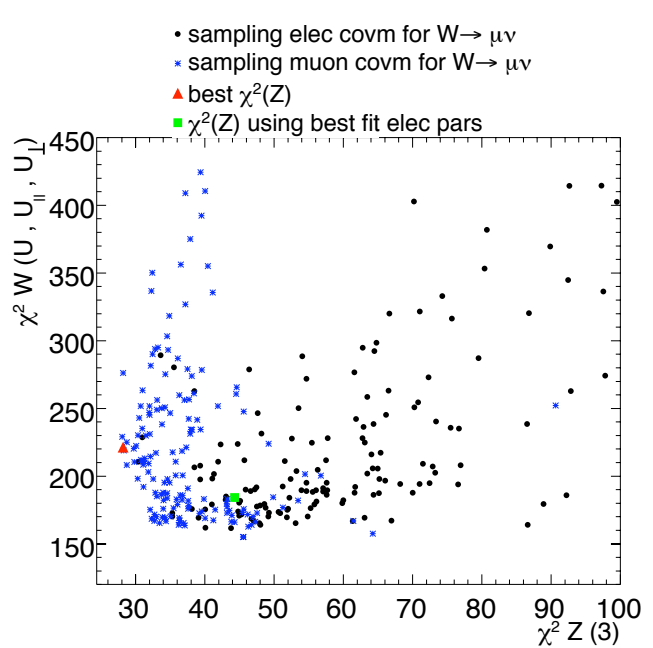

(b)

Figure 7.34: The spread of the $\chi^{2}$ of three $\mathrm{W}$ recoil distributions and the three fitted $\mathrm{Z}$ distributions when the electron and the muon covariance matrix are sampled in turn. 


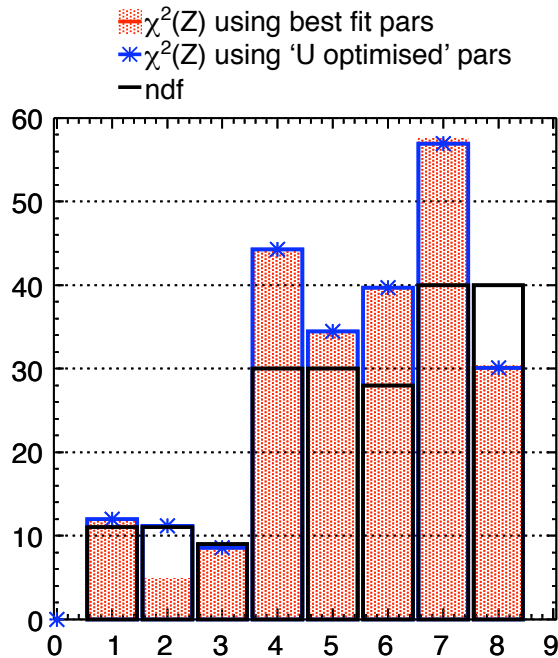

$$
\begin{aligned}
& \text { Key (x axis): } \\
& 1=\left\langle U_{\mathrm{T}}\right\rangle \text { vs } \mathrm{p}_{\mathrm{T}}(\mathrm{Z}) \\
& 2=\sigma\left(U_{1}\right) v s p_{T}(Z) \\
& 3=\sigma\left(U_{2}\right) v s p_{T}(Z) \\
& 4=U \\
& 5=U_{1} \\
& 6=U_{2} \\
& 7=U_{\| I} \\
& 8=U_{\perp}
\end{aligned}
$$

Figure 7.35: The effect on the $\chi^{2}$ of some of the recoil distribution in $\mathrm{Z} \rightarrow e^{+} e^{-}$events when using the recoil parameters optimised on the $U$ distribution.

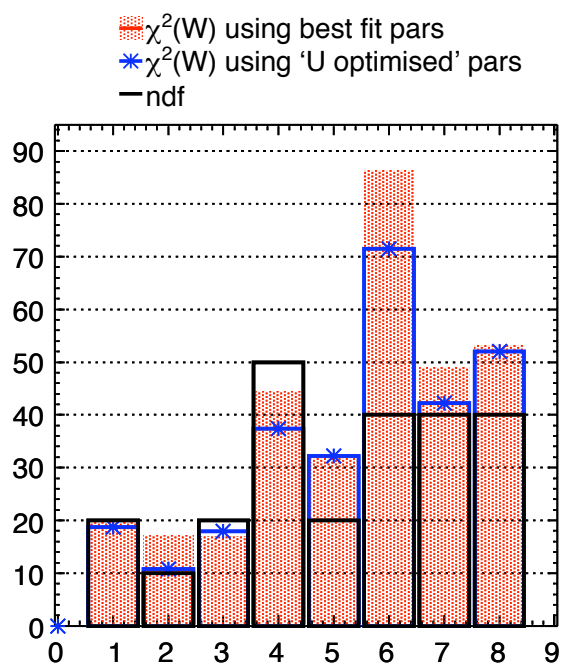

Key (x axis):
$1=\left\langle U_{\|}\right\rangle$vs $\Delta \phi(U, I)$
$2=\left\langle U_{\|}>\right.$vs $M_{T}$
$3=\left\langle U_{\|}\right\rangle$vs $U$
$4=E_{T}$
$5=\sigma\left(U_{\perp}\right)$ vs $U$
$6=U$
$7=U_{\|}$
$8=U_{\perp}$

Figure 7.36: The effect on the $\chi^{2}$ of some of the recoil distribution in $\mathrm{W} \rightarrow e \nu$ events when using the recoil parameters optimised on the $U$ distribution. 


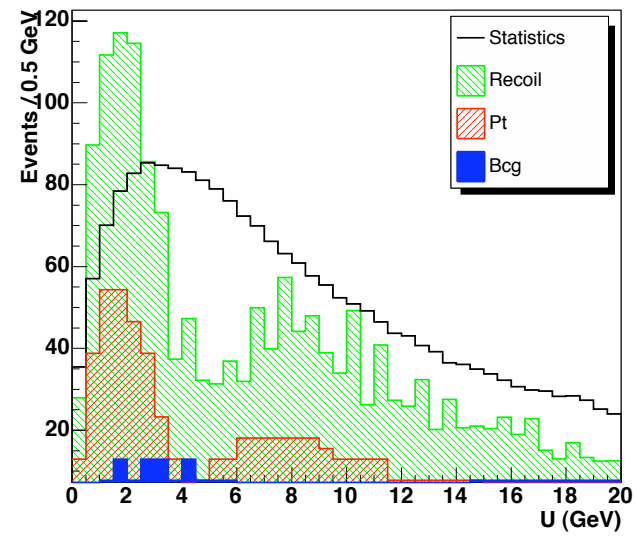

(a)

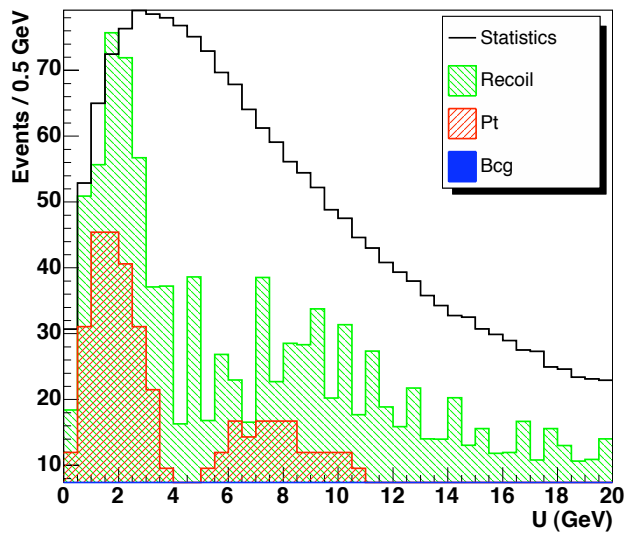

(b)

Figure 7.37: The uncertainty on the shape of the U distribution from statistics, the error on the recoil parameters (Recoil), boson $p_{\mathrm{T}}$ determination (Pt) and backgrounds (Bcg) for (a) $\mathrm{W} \rightarrow e \nu$ and (b) $\mathrm{W} \rightarrow \mu \nu$ events in the $\mathrm{W}$ width analysis.

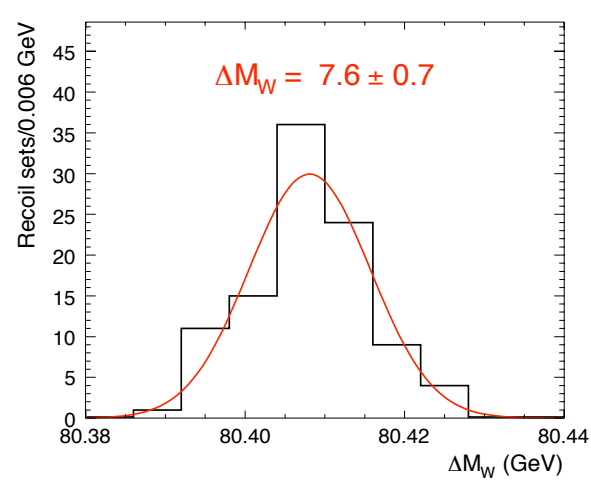

(a)

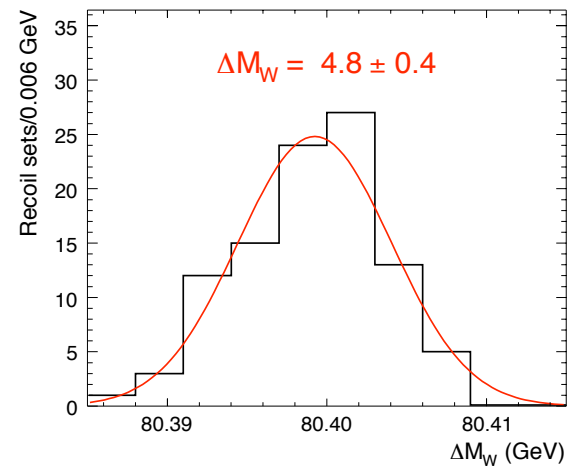

(b)

Figure 7.38: Spread of $M_{W}$ values obtained when fitting to $M_{T}$ distributions obtained using different sets of recoil parameters obtained from sampling the $6 \times 6$ covariance matrix in (a) $\mathrm{W} \rightarrow e \nu$ and (b) W $\rightarrow \mu \nu$ events in the $\mathrm{W}$ mass analysis. 


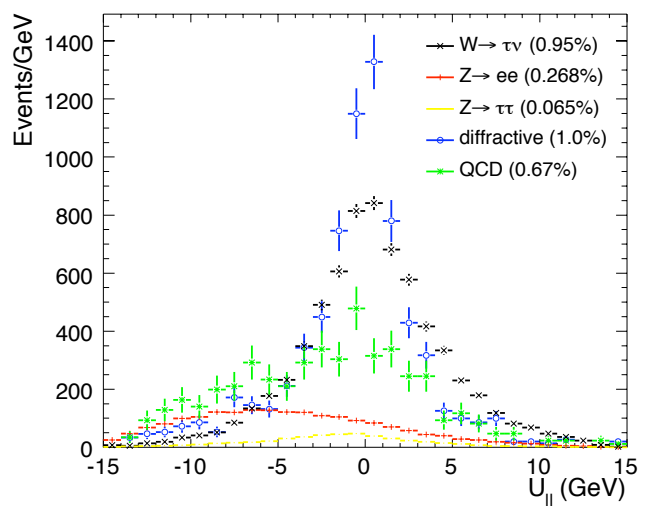

Figure 7.39: The $U_{\|}$distribution for backgrounds contributing to the electron channel in the W mass analysis. 


\section{Chapter 8}

\section{Recoil in Central-Plug Events}

The simulation of the recoil described in the previous chapter involves a parametric model, the parameters of which are obtained from fits to $\mathrm{Z} \rightarrow l^{+} l^{-}$events where both leptons are required to be reconstructed in the central rapidity region of the calorimeter. This is not always the case in $\mathrm{W} \rightarrow l \nu$ events, where the 'central' requirement can only be made on the charged lepton and the neutrino can go anywhere in the detector. Since the recoil in $\mathrm{W} \rightarrow l \nu$ events is simulated by tuning the recoil parameterisation on $\mathrm{Z} \rightarrow l^{+} l^{-}$events, this difference in their topology can introduce a potential bias in the recoil.

A generator level study using CdfSim found that in approximately $70 \%$ of $\mathrm{W} \rightarrow l \nu$ events the neutrino has a $|\eta|$ less than 1.0. The $\mathrm{W} \rightarrow l \nu$ samples can therefore be thought to comprise of $70 \% \mathrm{CC}$ (both leptons reconstructed in the central region) events and the remaining $30 \%$ can be attributed to $\mathrm{CP}$ (charged lepton is reconstructed in the central region and neutrino is in the plug calorimeter) events. The recoil in $\mathrm{CP}$ events may be different to that in $\mathrm{CC}$ events. It is therefore instructive to fit for the recoil in $\mathrm{CP} \mathrm{Z} \rightarrow e^{+} e^{-}$ events and make a quantitative comparison between the recoil parameters obtained from this fit to those obtained from the fit to $\mathrm{CC} \mathrm{Z} \rightarrow e^{+} e^{-}$events (described in the previous chapter).

This chapter will describe the reconstruction and simulation of $\mathrm{CP} \mathrm{Z} \rightarrow e^{+} e^{-}$events and the parameterisation of the recoil, concluding with a comparison with the recoil in $\mathrm{CC} \mathrm{Z} \rightarrow e^{+} e^{-}$events. 


\subsection{Event Selection}

$\mathrm{CP} \mathrm{Z} \rightarrow e^{+} e^{-}$events are selected by requiring one electron to be reconstructed in the central calorimeter and one in the plug calorimeter. The selection requirements for the central electron are the same as those used for $\mathrm{CC} \mathrm{Z} \rightarrow e^{+} e^{-}$events in the $\mathrm{W}$ mass analysis, given in Table 4.1. It is desirable to make the same kinematic requirements on the plug electron as the two electrons in $\mathrm{CC} \mathrm{Z}$ events and the missing energy in $\mathrm{W}$ events so there is negligible kinematic bias when comparisons are made between the different recoils. The plug calorimeter has a different granularity to the central calorimeter and the identification variables and fiducial requirements used to select an electron in the PEM are described in the following:

- $\chi_{3 \times 3}^{2}$ : this variable compares the energy distribution in the $3 \times 3$ towers around the seed tower to the distribution from test beam electrons. The best fit position for the centre of the tower and a $\chi^{2}$ are returned.

- Fiducial : the plug electron is required to be reconstructed in a well instrumented region of the detector. The PEM cluster is required to lie in the region $1.2<|\eta|<$ 2.8 in the calorimeter. It is also required to be away from the boundaries between the central and plug calorimeters.

- Isolation : this is defined as the energy, $E^{\text {iso }}$, in a cone of radius $\Delta R=\sqrt{\Delta \eta^{2}+\Delta \phi^{2}} \leq$ 0.4 around the electron cluster excluding the electron cluster energy.

A summary of the kinematic and identification cuts used to select a plug electron is given in Table 8.1.

The cuts used to select CP events are similar to those in Table 4.5 except the opposite sign cut is not made for CP events and the recoil cut is not applied since the recoil is being studied. The event yield obtained for $\mathrm{CP} \mathrm{Z} \rightarrow e^{+} e^{-}$events using this selection criteria is 45,549 , compared to a $\mathrm{CC} \mathrm{Z} \rightarrow e^{+} e^{-}$event yield of 33,039 . 


\begin{tabular}{|l|l|}
\hline Variable & Cut value \\
\hline \hline$E_{\mathrm{T}}$ & $>30 \mathrm{GeV}$ \\
$\left|z_{0}\right|$ & $<60 \mathrm{~cm}$ \\
$\chi_{3 \times 3}^{2}$ & $<10$ \\
$E^{\text {iso }}$ & $<4 \mathrm{GeV}$ \\
Fiducial & $1.2<|\eta|<2.8$ \\
\hline
\end{tabular}

Table 8.1: Selection criteria for plug electrons in $\mathrm{CP} \mathrm{Z} \rightarrow e^{+} e^{-}$events.

\subsection{Event Reconstruction}

The energy of the plug electron is reconstructed using the cluster energy in the PEM. The first layer of the PEM is referred to as the plug pre-radiator (PPR) and is read out separately. It is designed to correct the energy of particles that shower early in the calorimeter and can also be used to distinguish between electrons/photons and hadrons. The energy deposited by a plug electron in the PPR is added to the shower energy in the PEM.

The transverse energy is calculated using the $z$ position of the central electron track in the event. The $z$-vertex of the event is also reconstructed as the $z$ position of the central electron track. The recoil is calculated as before with the same number of towers excluded from the recoil sum for the central electron as described in the previous chapter. The plug electron has a different knockout region and this is explained in detail below.

\subsubsection{Plug Knockout Region}

The plug calorimeter has a different and more complex granularity compared to the central calorimeter. The calorimeter is azimuthally segmented into 48 wedges at lower values of $|\eta|$, with each wedge subtending $7.5^{0}$ in $\phi$. At higher values of $|\eta|$, the segmentation halves to resemble that of the CEM, with 24 wedges, each subtending $15^{0}$ in $\phi$. A schematic diagram of the segmentation of the plug calorimeter is shown in Figure 8.1. Whereas the towers at tower number $(i \eta)=10,11$ have a physical $\phi$ segmentation of $7.5^{0}$, they are 


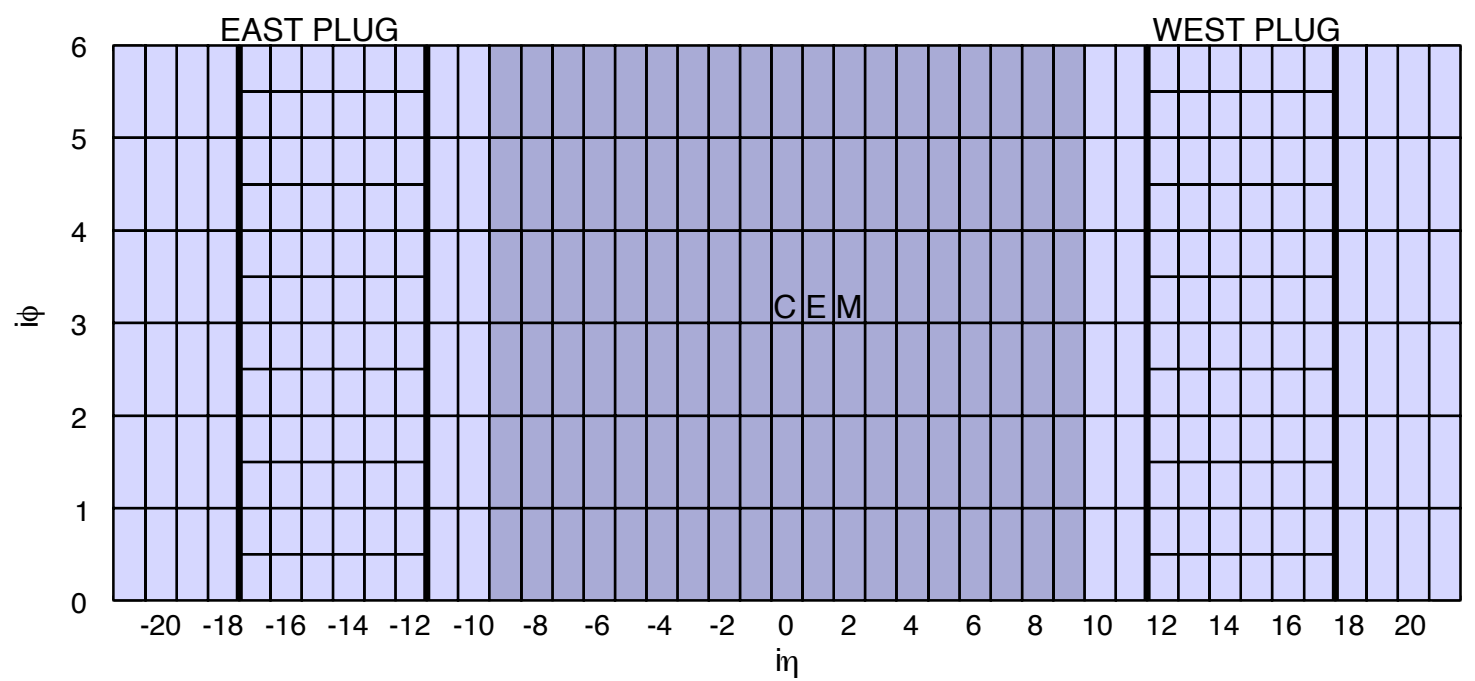

Figure 8.1: The segmentation in $(\eta, \phi)$ of the plug calorimeter (only a quarter of the detector is shown in $\phi)$.

overshadowed by towers in the WHA calorimeter which are azimuthally segmented into $15^{\circ}$ wedges. Therefore, in this $\eta$ region, for all physics purposes, the towers are defined to be $15^{0}$ in $\phi$ so that the plug and WHA have consistent $(\eta, \phi)$ segmentation.

The number of towers that need to be included in the knockout region for the plug electron depends strongly on the $\eta$ of the tower. There are a number of scenarios for which a separate knockout region needs to be considered depending on where the plug electron is in the calorimeter and they fall into three broad categories:

- 48-wedge region : The plug electron is reconstructed in a tower that subtends $7.5^{0}$ in $\phi$ and there are therefore 48 wedges in each $\phi$ slice. More towers will need to be knocked out since the towers are smaller. The neighbouring towers also have the same azimuthal segmentation and the electron tower is therefore not on the boundary between a 24-wedge and 48-wedge region. From Figure 8.1 a plug electron reconstructed in towers 13 to 16 satisfies this category. 
- 24-wedge region : The plug electron is in a tower that subtends $15^{0}$ in $\phi$ and there are therefore 24 wedges in each $\phi$ slice. The neighbouring towers also have the same azimuthal segmentation and the electron tower is therefore not on the boundary between a 24-wedge and 48-wedge region. From Figure 8.1 a plug electron reconstructed in towers 19 and 20 satisfies this category.

- boundary : The plug electron is reconstructed in a tower that is on the boundary between a 24-wedge region and a 48-wedge region. This is true for towers 11, 12, 17 and 18 .

Events where the plug electron is reconstructed in the outer towers of the plug calorimeter, i.e towers 10 and 21 are rejected to avoid the electromagnetic shower extending over the edge of the PEM.

The knockout region for a plug electron that is in the 24-wedge and 48-wedge regions is shown in Figures 8.2 and 8.3 respectively. For the 24-wedge region, 9 towers are excluded for both the plug electromagnetic and hadronic calorimeter and in the 48-wedge region, 15 towers are knocked out. In cases where the plug electron is on the boundary between the 24-wedge and 48-wedge regions, the towers excluded from the recoil calculation are shown in Figures 8.4-8.7 for the boundaries in the east half of the plug calorimeter. The same number of towers are knocked out for the west half of the calorimeter. A summary of the number of towers comprising the knockout region depending on the tower number of the plug electron is given in Table 8.2.

\begin{tabular}{|l|c|}
\hline Tower number $i \eta$ & Number of towers \\
\hline \hline $13<|i \eta|<16$ & 15 \\
$|i \eta|=19,20$ & 9 \\
$|i \eta|=11,18$ & 12 \\
$|i \eta|=12,17$ & 17 \\
\hline
\end{tabular}

Table 8.2: The number of towers in the knockout region depending on the tower of the plug electron. 

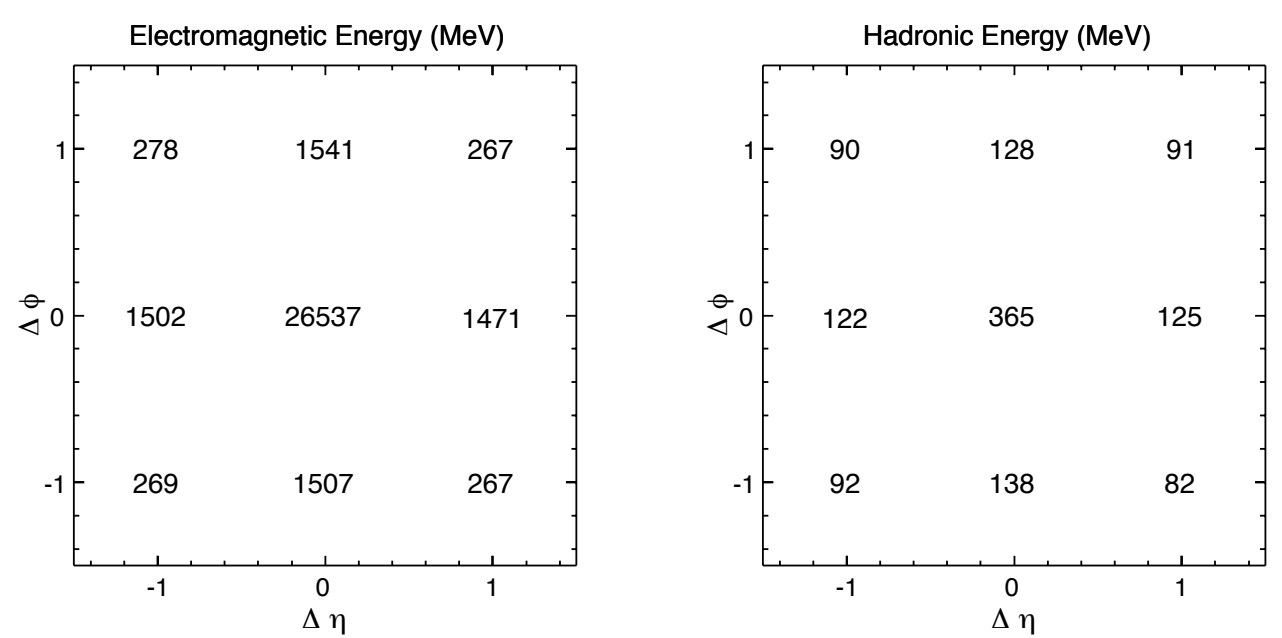

Figure 8.2: The energy deposited (in $\mathrm{MeV}$ ) in the knockout region for events where the plug electron is reconstructed in a region of the calorimeter that has a 24-wedge segmentation in $\phi$.
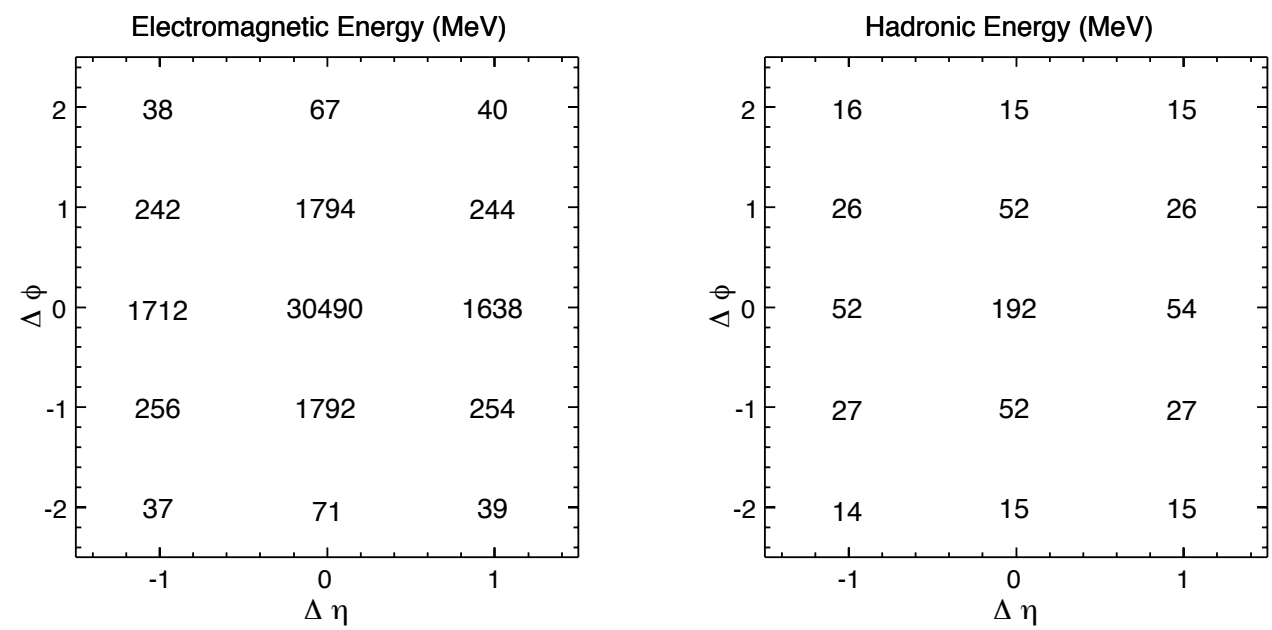

Figure 8.3: The energy deposited (in $\mathrm{MeV}$ ) in the knockout region for events where the plug electron is reconstructed in a region of the calorimeter that has a 48-wedge segmentation in $\phi$. 

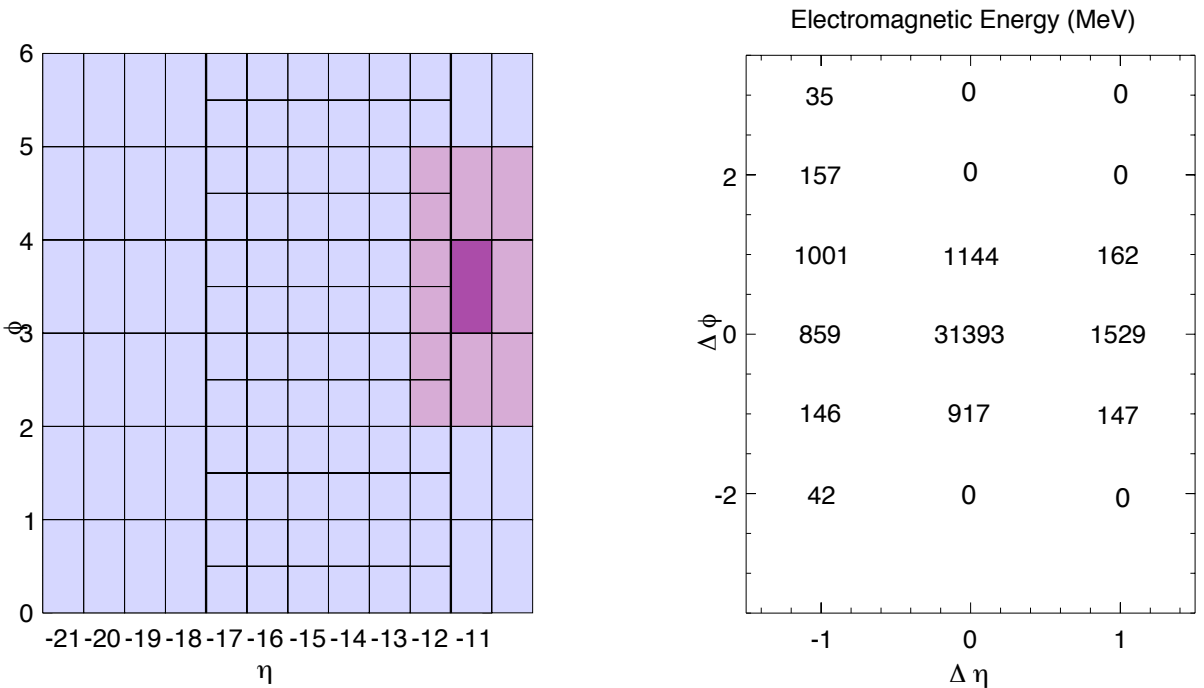

Figure 8.4: The energy deposited (in $\mathrm{MeV}$ ) in the knockout region for a plug electron reconstructed in tower number -11 where the plug electron is in the 24 -wedge $\phi$ slice but on the boundary between a 24 and 48-wedge region.

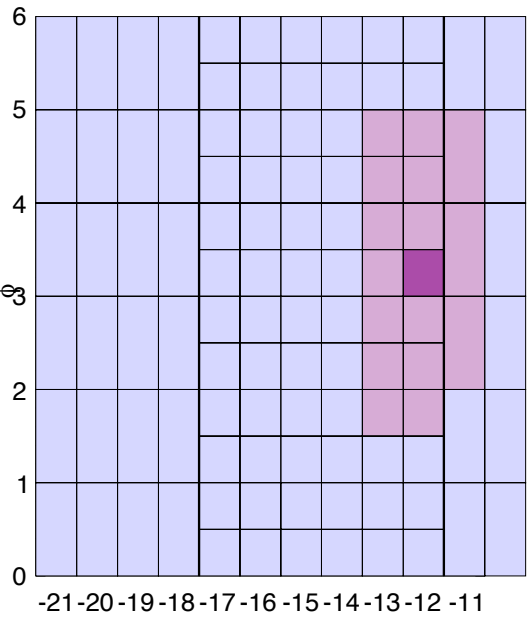

$\eta$
Electromagnetic Energy (MeV)

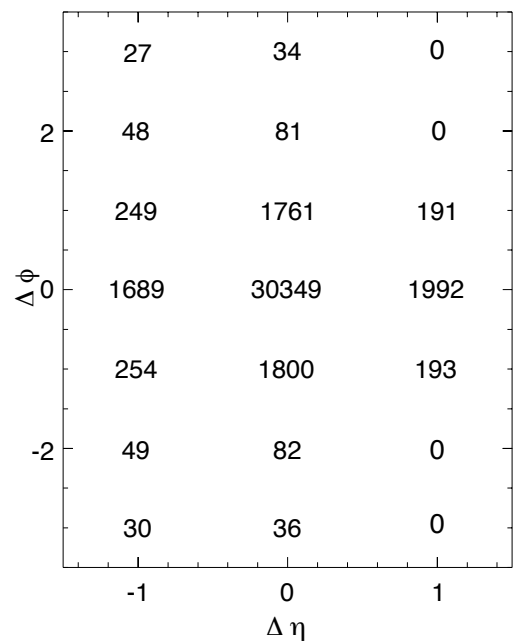

Figure 8.5: The energy deposited (in $\mathrm{MeV}$ ) in the knockout region for a plug electron reconstructed in tower number -12 where the plug electron is in the 48 -wedge $\phi$ slice but on the boundary between a 24 and 48-wedge region. 


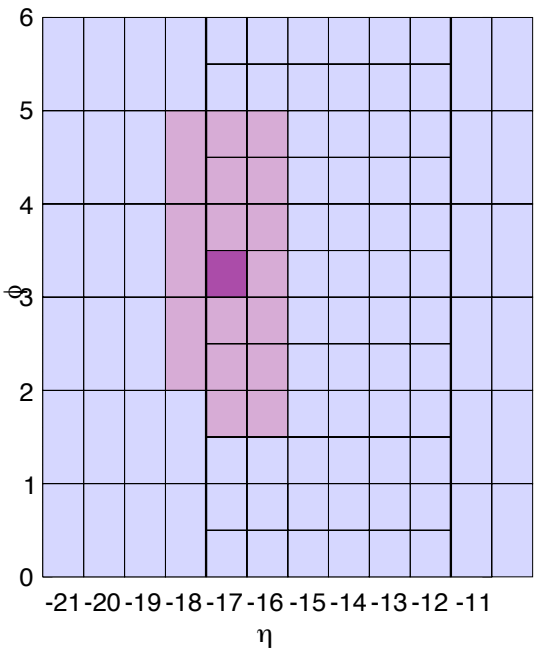

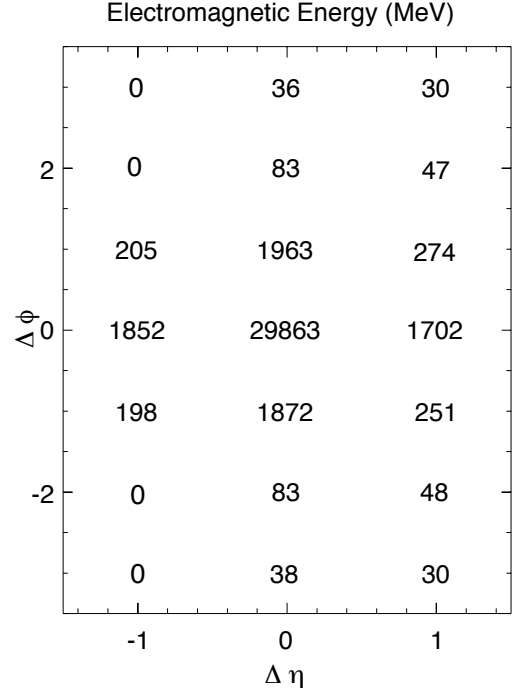

Figure 8.6: The energy deposited (in $\mathrm{MeV}$ ) in the knockout region for a plug electron reconstructed in tower number -17 tower where the plug electron is in the 48 -wedge $\phi$ slice but on the boundary between a 24 and 48-wedge region.
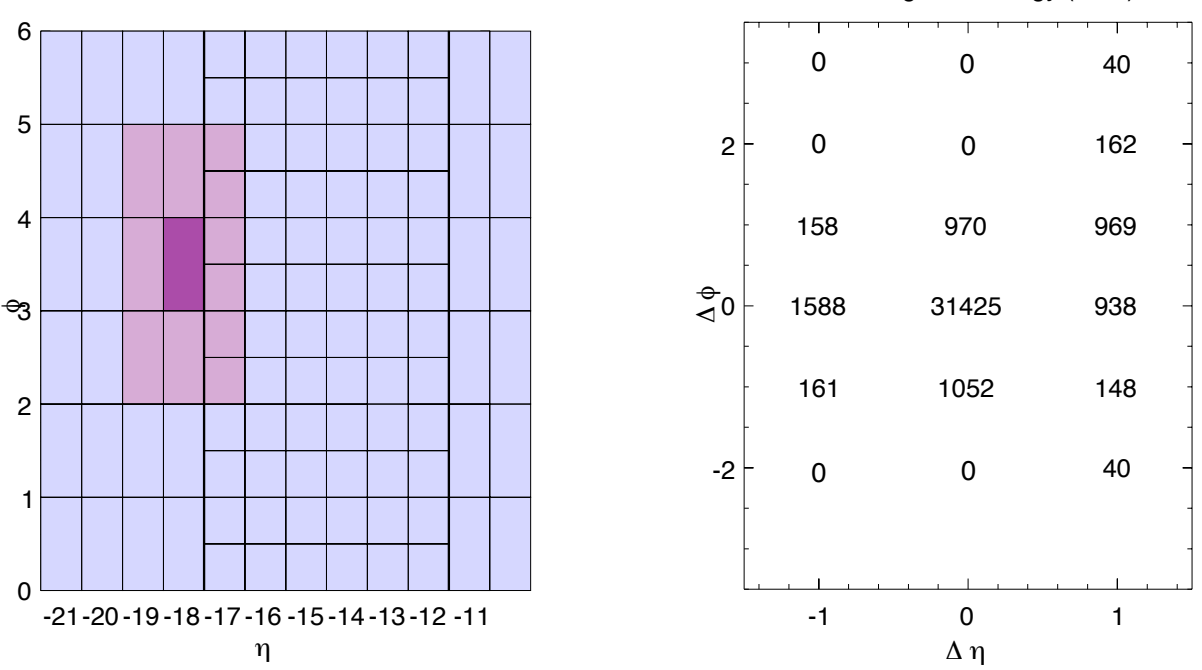

Figure 8.7: The energy deposited (in $\mathrm{MeV}$ ) in the knockout region for a plug electron reconstructed in tower number -18 where the plug electron is in the 24 -wedge $\phi$ slice but on the boundary between a 24 and 48-wedge region. 


\subsection{Event Simulation}

The simulation of CP events involves propagating one electron through the detector simulation to the central calorimeter and propagating the other to the plug calorimeter. The simulation of the central electron proceeds as described in Chapter 6. The simulation of the plug electron also proceeds in the same way until the calorimeter.

The PEM has a different response and resolution to incoming electrons/photons compared to the CEM. Whereas the CEM scale and resolution determined in Chapter 6 can be applied to the energy of the central electron, the PEM scale and resolution need to be separately determined.

The resolution of the PEM calorimeter is described by

$$
\sigma_{E} / E=14.4 \% / \sqrt{E}(\mathrm{GeV}) \oplus \kappa
$$

where $14.4 \%$ is the stochastic term for the plug calorimeter obtained from test beam data.

The plug electron $E_{T}$ distribution shows some dependence on the plug $\eta$. The scale $\left(S^{\mathrm{PEM}}\right)$ and resolution for the PEM is therefore obtained separately for four $\eta$ regions; $-2.8<\eta<-1.6,-1.6<\eta<-1.2,1.2<\eta<1.6$ and $1.6<\eta<2.8$. Simulation templates of the $\mathrm{Z}$ boson invariant mass distribution are produced with different input values for $S^{\mathrm{PEM}}$ and for each $\eta$ region in the calorimeter. The templates are used to fit to the $\mathrm{Z}$ mass distribution in $\mathrm{Z} \rightarrow e^{+} e^{-}$data for each calorimeter $\eta$ region to obtain the value of $S^{\mathrm{PEM}}$ that minimises the $\chi^{2}$ of the invariant mass peak in the $\mathrm{M}_{\mathrm{Z}}$ region, $86<\mathrm{M}_{\mathrm{Z}}<96$ GeV. The fits in each $\eta$ region are shown in Figure 8.8. The invariant mass distribution in the region $-1.6<\eta<-1.2$ does not describe the data well, however a discrepancy in the invariant mass distribution has a negligible effect on the recoil distributions.

The values for $\kappa$ are also found by fitting simulation templates of the invariant mass distribution with different input values for $\kappa$ to the data. The best fit values for $S^{\text {PEM }}$ and $\kappa$ for each region are shown in Table 8.3, where the value of kappa obtained for the 


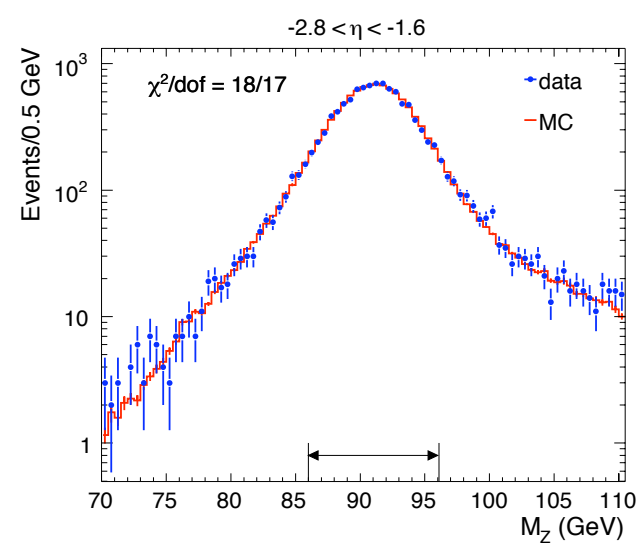

(a)

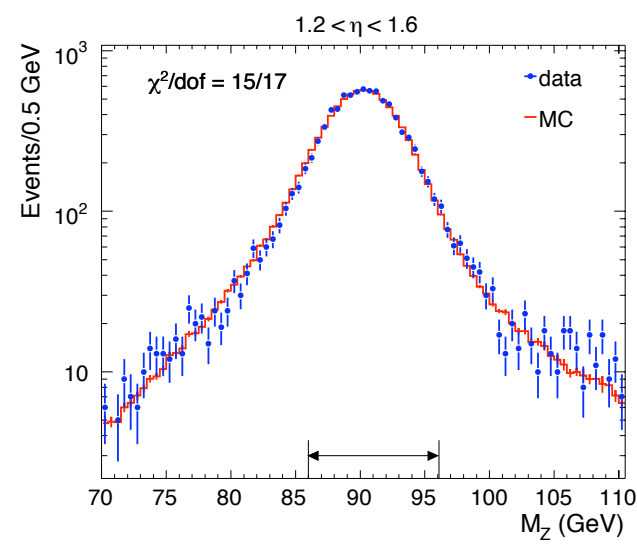

(c)

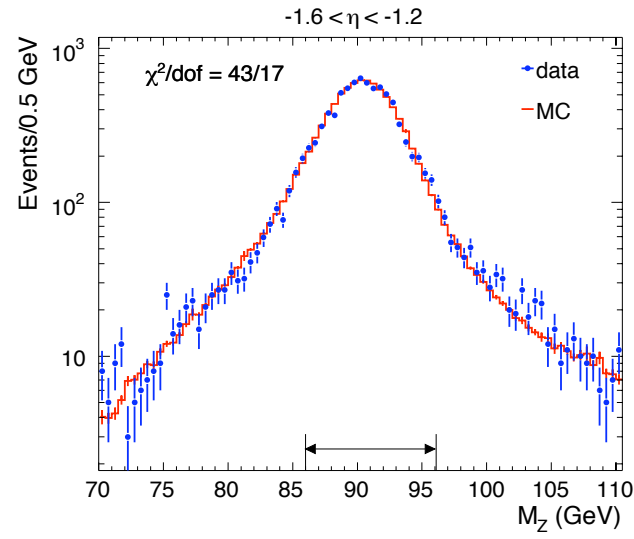

(b)

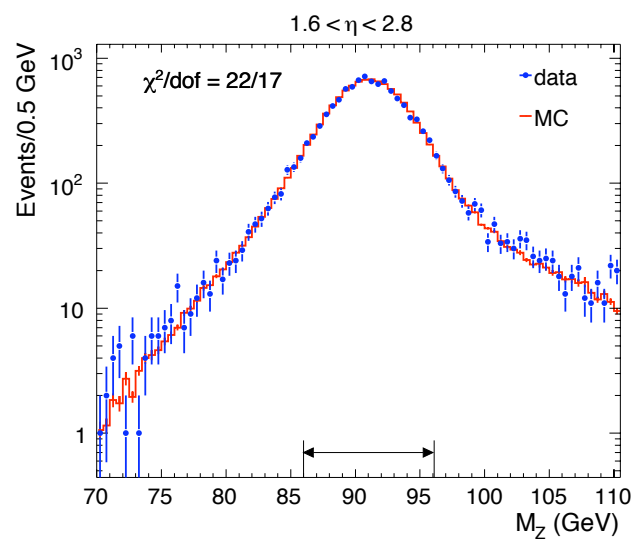

(d)

Figure 8.8: Fits to the invariant mass distribution in $\mathrm{CP} \mathrm{Z} \rightarrow e^{+} e^{-}$events for the plug electron in the region (a) $-2.8<\eta<-1.6$ (b) $-1.6<\eta<-1.2$ (c) $1.2<\eta<1.6$ and (d) $1.6<\eta<2.8$.

region $-1.6<\eta<-1.2$ is different from the other values. In addition, after applying the scale factors in Table 8.3, an overall PEM scale factor is obtained such that the inclusive $\mathrm{Z}$ invariant mass distribution shows good agreement between data and simulation. This overall scale factor is found to be $0.9986 \pm 0.0005$. The inclusive $\mathrm{M}_{\mathrm{Z}}$ distribution is shown in Figure 8.9. The plug electron $\eta$ and $\phi$ distributions in data and simulation are shown in Figure 8.10. The geometric acceptance of the electron in the plug calorimeter is well described by the simulation. The rapidity of the $\mathrm{Z}$ boson and the $E_{T}$ of the plug electron is shown in Figure 8.11.

The efficiency of the identification cuts $\left(\chi_{3 \times 3}^{2}, E_{\mathrm{had}} / E_{\mathrm{em}}\right.$ and isolation $)$ used to select 


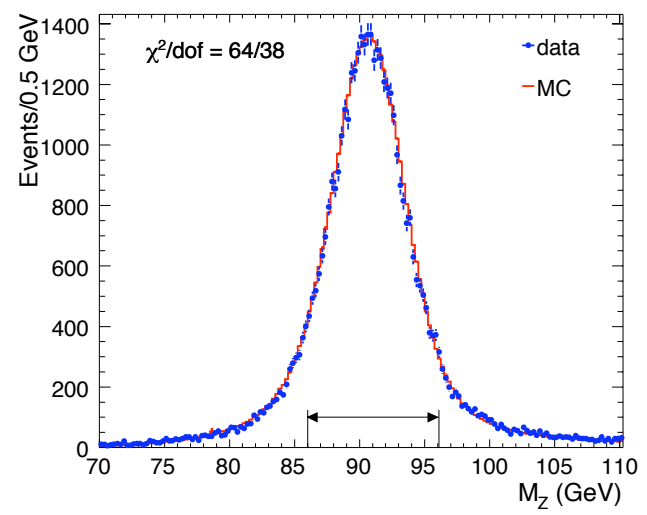

Figure 8.9: The fit to the invariant mass distribution of the $\mathrm{Z}$ boson to obtain an overall $S^{\mathrm{PEM}}$.

\begin{tabular}{|c|c|c|}
\hline$\eta$ region & $S^{\text {PEM }}$ & $\kappa$ \\
\hline \hline$-2.8<\eta<-1.6$ & $1.0089 \pm 0.0009$ & $0.031 \pm 0.002$ \\
$-1.6<\eta<-1.2$ & $0.9931 \pm 0.0008$ & $0.022 \pm 0.002$ \\
$1.2<\eta<1.6$ & $0.9905 \pm 0.0010$ & $0.030 \pm 0.002$ \\
$1.6<\eta<2.8$ & $1.0075 \pm 0.0009$ & $0.032 \pm 0.002$ \\
\hline
\end{tabular}

Table 8.3: The PEM scale and resolution obtained by fitting to the invariant mass of the $\mathrm{Z}$ boson in four $\eta$ regions.

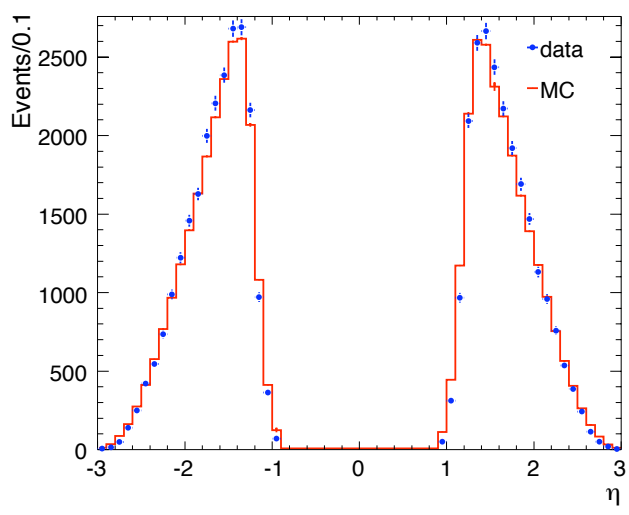

(a)

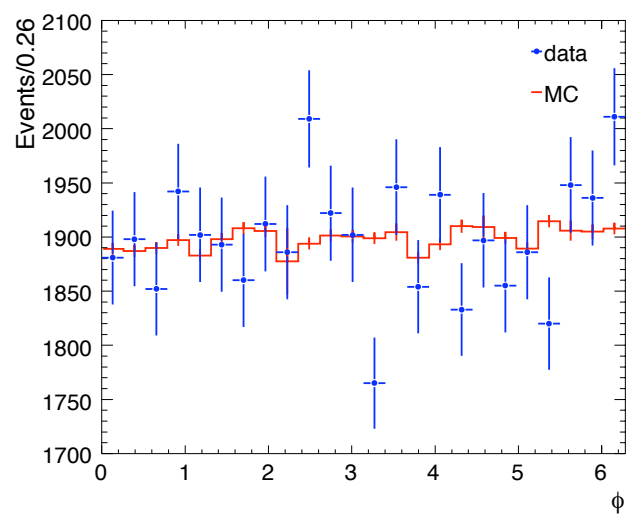

(b)

Figure 8.10: The plug electron (a) $\eta$ and (b) $\phi$ distributions for data and simulation.

plug electrons has a dependence on $U_{\|}$and the instantaneous luminosity of the event, which may bias the recoil distributions. The efficiency is defined as the subset of events where a plug electron candidate passes the following identification cuts; $\chi_{3 \times 3}^{2}, E_{\mathrm{had}} / E_{\mathrm{em}}$ 


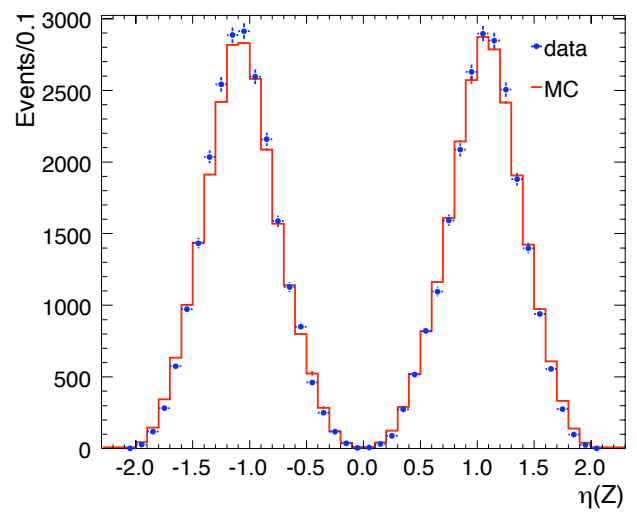

(a)

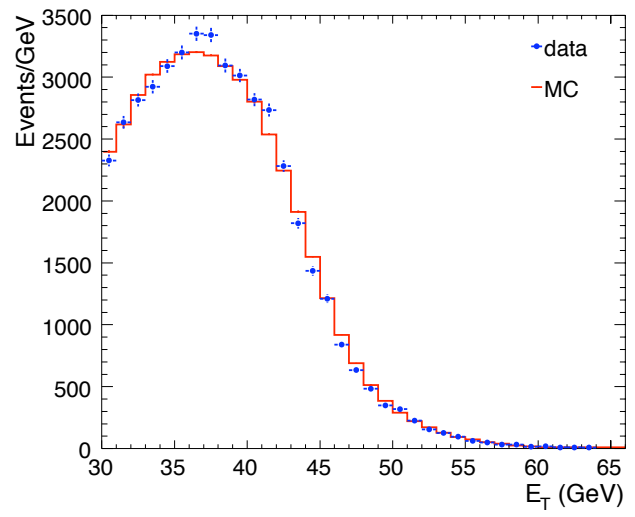

(b)

Figure 8.11: (a) The $\mathrm{Z}$ boson rapidity distribution in CP data and simulation events. (b) The plug electron $E_{T}$ distribution.

and isolation compared to the events where the plug electron passes the identification and kinematic cuts. The central electron is required to pass the default selection. The ID efficiency of the plug electron decreases as a function of Run Period, shown in Figure 8.12(a). Each Run Period contains a series of runs collected over a period of time, with higher Run Periods corresponding to later runs. Each period contains a range of instantaneous luminosity, the average of which increases with time. This therefore shows the dependence of the ID efficiency on luminosity. At higher luminosities, there is an increase in the number of additional interactions in the same beam crossing, resulting in more activity in the event. This increase in underlying energy can reduce the efficiency of certain variables used to select electrons. The $\chi_{3 \times 3}^{2}$ variable is the main source of this decrease in efficiency. The efficiency of $\chi_{3 \times 3}^{2}$ is shown as a function of Run Period in Figure $8.12(\mathrm{~b})$. Since $\chi_{3 \times 3}^{2}$ is a quantitatve comparison between the energy distribution of the plug EM object and the energy distribution from test beam electrons, the more underlying energy there is in the calorimeter, the less efficient this selection cut becomes. The plug ID efficiency depends on the instantaneous luminosity of the event as shown in Figure 8.13. The efficiency drops by $20 \%$ at high luminosity. This efficiency dependence on luminosity is included in the simulation. The plug ID efficiency also varies as a function 


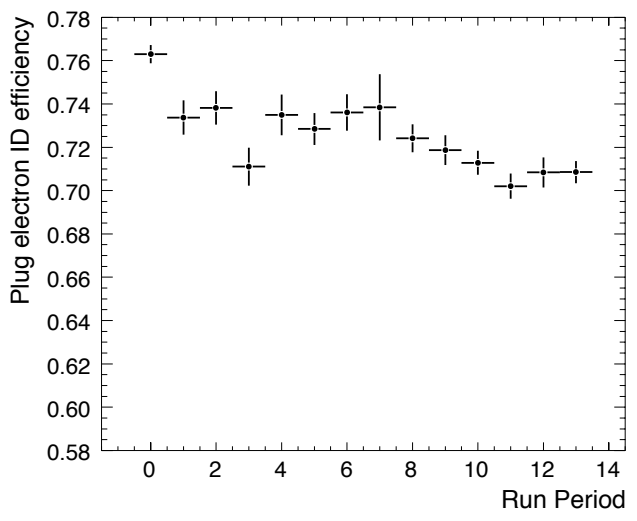

(a)

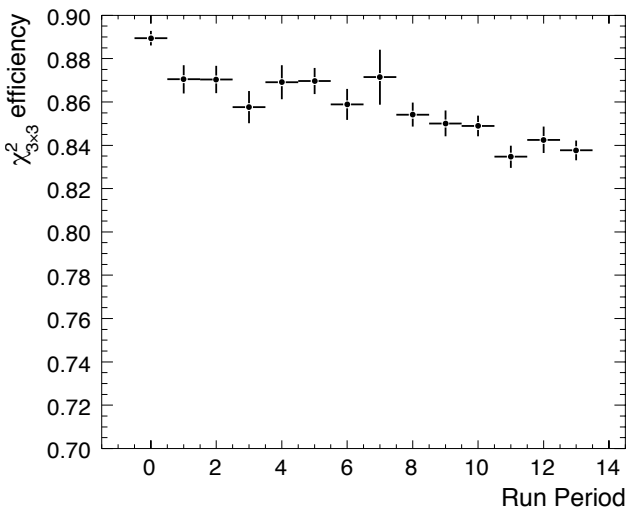

(b)

Figure 8.12: (a) The plug electron ID efficiency as a function of Run Period. (b) The efficiency of $\chi_{3 \times 3}^{2}$ as a function of Run Period.

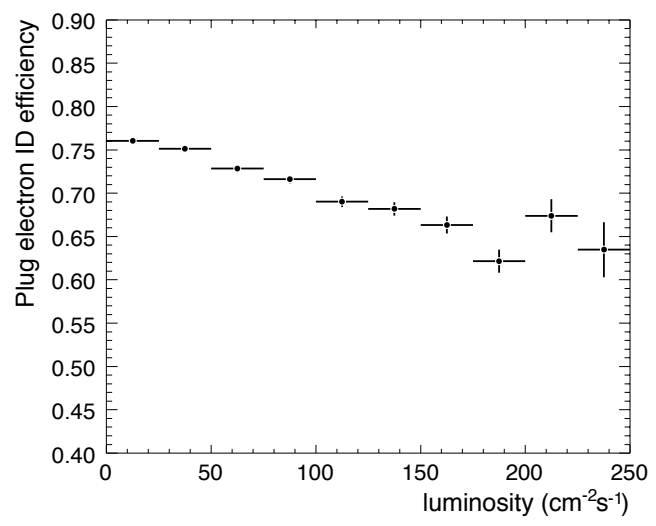

Figure 8.13: The plug electron ID efficiency as a function of instantaneous luminosity.

of $U_{\|}$. This dependence is taken from CdfSim, shown in Figure 8.14 and the functional form obtained by fitting to it is included in the simulation.

\subsection{Boson $p_{\mathrm{T}}$ Fit}

The $\mathrm{Z}$ boson in $\mathrm{CP}$ events has a different transverse momentum $\left(p_{\mathrm{T}}\right)$ and rapidity distribution (shown in Figure 8.11) to that in CC events. The same procedure as described in Section 5.2 is used to fit to the $p_{\mathrm{T}}$ distribution in CP events. The mean absolute rapidity of the $\mathrm{Z}$ boson in $\mathrm{CP}$ events is 1.02. The rapidity reweighting function is therefore mod- 


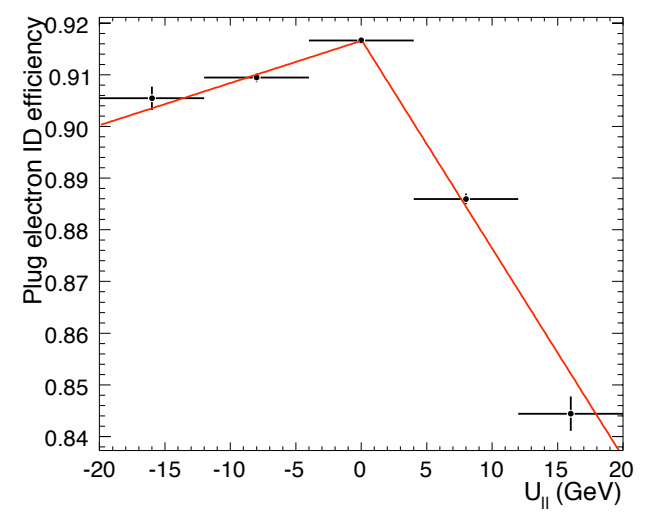

Figure 8.14: The plug electron ID efficiency as a function of $U_{\|}$.

ified to be $\frac{d \sigma}{d p_{T}}(|Y|=1.02) / \frac{d^{2} \sigma}{d Y d p_{T}}(|Y|=Y)$. A fit to the boson $p_{\mathrm{T}}$ in $\mathrm{Z} \rightarrow e^{+} e^{-}$data is performed whilst varying parameter $g_{2}$ from the BLNY functional form since it is most sensitive to the $p_{\mathrm{T}}$ distribution and keeping the other 3 parameters fixed to their global fit values given in [49]. The result of the fit is shown in Figure 8.15. The value of $g_{2}$ that minimises the $\chi^{2}$ for this fit is

$$
g_{2}=0.622 \pm 0.018
$$

This is consistent with the $g_{2}$ value obtained from a fit to the $\mathrm{CC} \mathrm{Z}$ boson $p_{\mathrm{T}}$ distribution which gave $g_{2}=0.637 \pm 0.013$. Although the $p_{\mathrm{T}}$ distribution is not well described, this discrepancy is found to have a negligible effect on the recoil distributions used in the recoil fit.

\subsection{Knockout Region Simulation}

The towers in the plug knockout region excluded from the recoil calculation contain recoil energy in addition to the energy associated with the plug electron and bremsstrahlung. In contrast to the recoil energy in the knockout region for the central electron where there is a weak dependence on the $\eta$ of the calorimeter tower, for the plug electron the most significant dependence of the recoil energy is on tower $\eta$. Each of the scenarios mentioned 


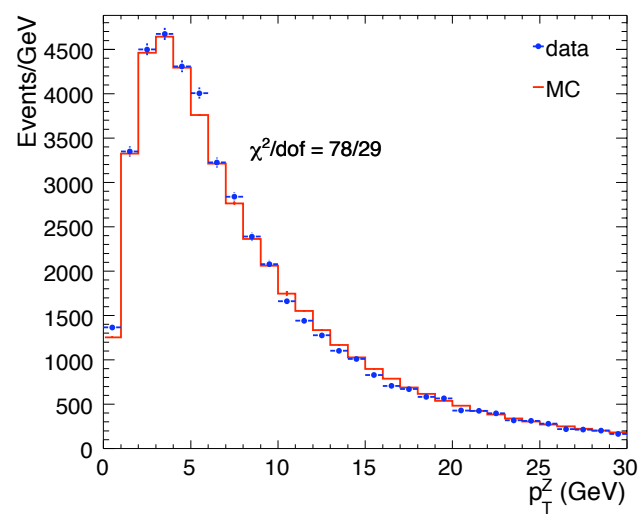

Figure 8.15: The $\mathrm{Z}$ boson $p_{\mathrm{T}}$ distribution simulation and data for $\mathrm{CP} \mathrm{Z} \rightarrow e^{+} e^{-}$events.

in Section 8.2.1, where the plug electron is reconstructed in regions of the calorimeter that have different $\phi$ segmentation, are studied. A pseudo-cluster with the same number of towers as those in the plug knockout region and with the centre of the cluster at the same $\eta$ as the plug electron is defined in the region $90^{\circ}$ in $\phi$ from the plug electron cluster. The distribution of the recoil energy in the pseudo-clusters corresponding to a plug electron reconstructed in the 48-wedge region, 24-wedge region and the boundary between the two is shown in Figure 8.16.

Pseudo-clusters at high $|\eta|$ tend to have more overlapping recoil energy because these towers are more forward in the detector and receive energy contributions from the low $p_{\mathrm{T}}$ remnants of the $p \bar{p}$ collision. This can be seen in Figure 8.17 which shows the percentage of events with no overlapping recoil energy in the cluster as a function of the tower $\eta$. The numbers are obtained from studying the zero fraction bin at $\log _{10} \mathrm{E}_{\mathrm{EM}}=-4.0$ for the distributions in Figure 8.16. At higher $|\eta|$, there are fewer events with no recoil energy in the pseudo-clusters.

The recoil energy in the knockout region is simulated by sampling from one of the distributions in Figure 8.16 depending on which category the tower $\eta$ of the plug electron falls into. This sampled energy is then subtracted from the recoil in the simulation to simulate the 'hole' in the recoil from removing the knockout region in the data. 

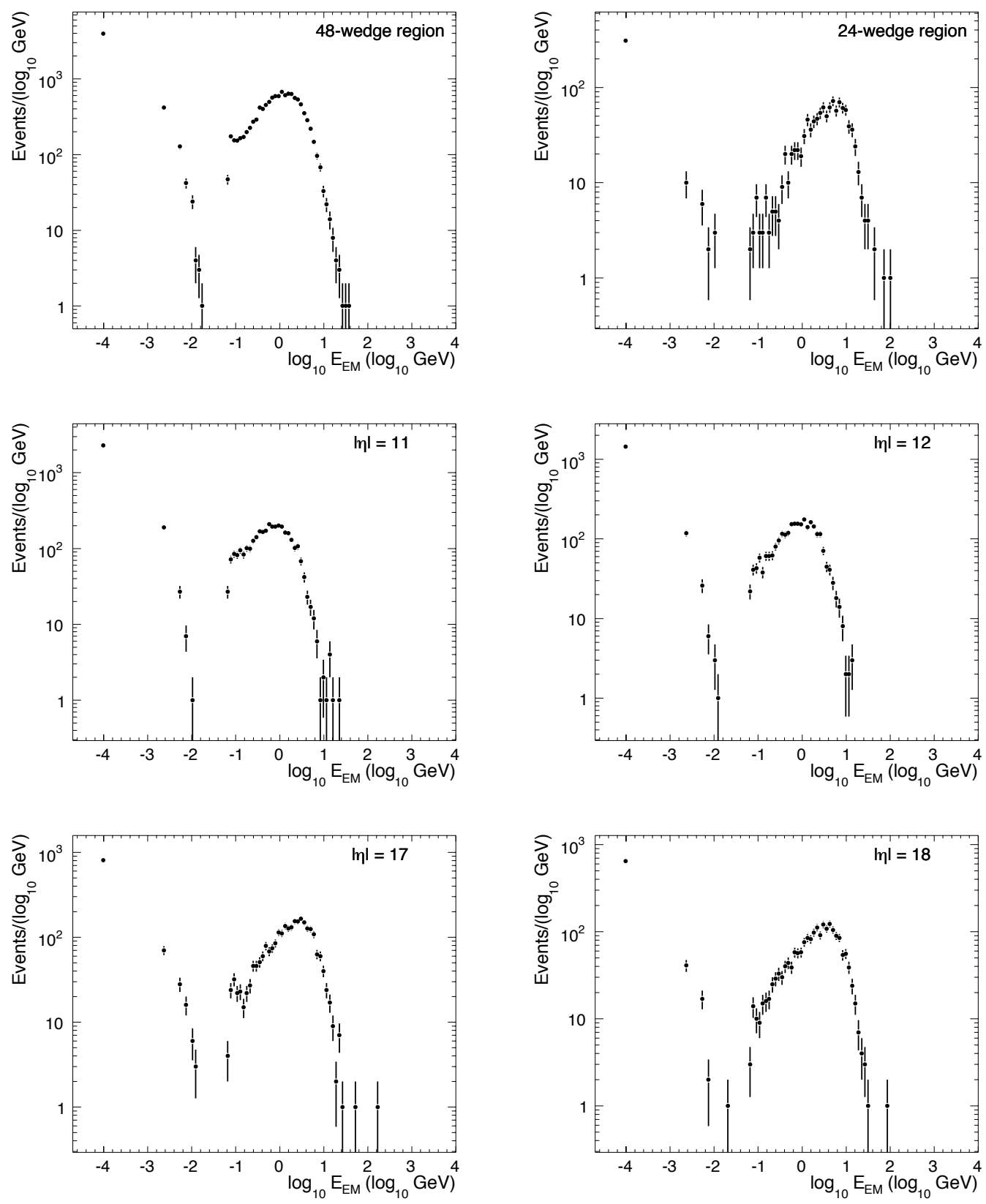

Figure 8.16: Underlying energy in a pseudo-cluster in a region orthogonal in $\phi$ but at the same $\eta$ as the plug electron in $\mathrm{CP} \mathrm{Z} \rightarrow e^{+} e^{-}$events.

\subsection{Backgrounds}

The event selection criteria with the high $E_{T}$ requirement on both electrons and lepton identification cuts produces a $\mathrm{CP} \mathrm{Z} \rightarrow e^{+} e^{-}$sample with high purity. However, there 


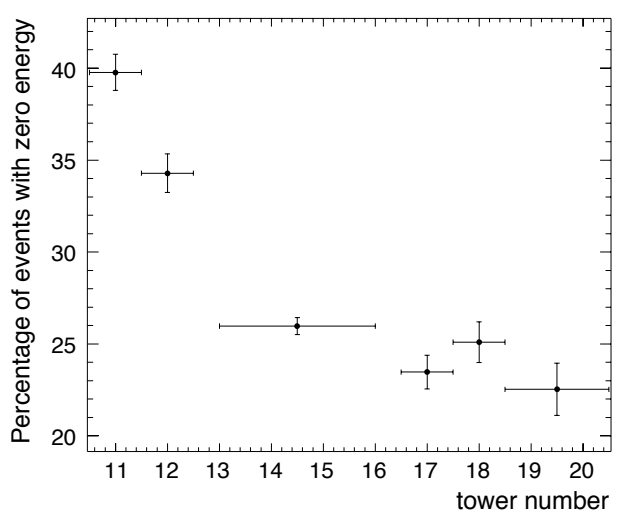

Figure 8.17: The percentage of events with no recoil energy in the pseudo-cluster as a function of tower number.

is some contamination from background events that can affect the recoil distributions.

The backgrounds can be divided into QCD background and non-QCD background, where the normalisation and shape of the QCD background is estimated using data and the electroweak backgrounds comprising the non-QCD background category are estimated using CdfSim.

\subsubsection{Non-QCD Backgrounds}

The electroweak backgrounds contributing to the $\mathrm{CP} \mathrm{Z} \rightarrow e^{+} e^{-}$sample are $\mathrm{Z} \rightarrow \tau^{+} \tau^{-}$ and $\mathrm{W}$ boson produced in association with jet(s) (W+jet events). The normalisation and shape of both backgrounds is estimated using large Monte Carlo samples that are generated with PyтHIA [64] and simulated using CdfSim.

\section{$\mathrm{Z} \rightarrow \tau \tau$}

$\mathrm{Z} \rightarrow \tau^{+} \tau^{-}$events where both $\tau$ leptons decay to an electron and a neutrino can mimic $\mathrm{CP} \mathrm{Z} \rightarrow e^{+} e^{-}$events. The fraction of $\mathrm{Z} \rightarrow \tau^{+} \tau^{-}$events in the $\mathrm{CP}$ sample is estimated by propagating a sample of such events obtained from CdfSim through the full event selection. A CdfSim sample of $\mathrm{CP} \mathrm{Z} \rightarrow e^{+} e^{-}$events is also generated and passed through the event selection. The proportion of $\mathrm{Z} \rightarrow \tau^{+} \tau^{-}$and $\mathrm{Z} \rightarrow e^{+} e^{-}$ 
events passing the selection criteria compared to the number of events generated is determined. This ratio of acceptances is then multiplied by the ratio of the $\mathrm{Z} \rightarrow \tau^{+} \tau^{-}$ to $\mathrm{Z} \rightarrow e^{+} e^{-}$cross-section times branching ratio. The fractional background is found to be $(0.040 \pm 0.004) \%$.

\section{$\mathrm{W}+$ jets}

$\mathrm{W}+$ jet events can mimic $\mathrm{CP} \mathrm{Z} \rightarrow e^{+} e^{-}$events if the $\mathrm{W}$ decays to an electron that passes the selection criteria and the jet fakes the other electron in the event. The $\mathrm{W}+$ jet background fraction is estimated by propagating a CdfSim sample of $\mathrm{W}+$ jet events through the $\mathrm{CP}$ event selection. The ratio of acceptance of $\mathrm{W}+$ jet to $\mathrm{Z} \rightarrow e^{+} e^{-}$events is then multiplied by the ratio of their corresponding cross-section times branching ratios. The NNLO calculation of the ratio of $\mathrm{W} \rightarrow l \nu$ to $\mathrm{Z} \rightarrow l^{+} l^{-}$ cross-section is; $R=\sigma(\mathrm{W} \rightarrow l \nu) / \sigma\left(\mathrm{Z} \rightarrow l^{+} l^{-}\right)=10.67 \pm 0.15$ [63] is used. The percentage of $\mathrm{W}+$ jet background in the $\mathrm{CP}$ sample is found to be $(0.16 \pm 0.07) \%$.

In addition to the above electroweak backgrounds, a diffractive background of $1 \%$ (described in more detail in Section 9.2) is also added to the CP simulation.

\subsubsection{QCD}

QCD or multi-jet events where two jets contain or fake an electron can pass the selection criteria and mimic a $\mathrm{CP} \mathrm{Z} \rightarrow e^{+} e^{-}$event. The amount of QCD background is estimated by fitting to the calorimeter isolation fraction distribution in the data, defined as the ratio of the calorimeter isolation energy and the electron transverse energy. A sample rich in QCD events is selected by requiring the plug electron to pass certain 'anti-electron' cuts, designed to select misidentified electrons. The central electron is required to pass all the default cuts. The following selection criteria is applied to the plug electron :

- The $E_{\mathrm{had}} / E_{\mathrm{em}}$ cut is removed. An anti $E_{\mathrm{had}} / E_{\mathrm{em}}$ cut is applied; $E_{\mathrm{had}} / E_{\mathrm{em}}>0.1$ and the maximum value is set to 0.125 . 
- The $\chi_{3 \times 3}^{2}$ cut is removed.

- The calorimeter isolation cut is relaxed and an anti calorimeter isolation cut is applied; $E^{\text {iso }}>4.0 \mathrm{GeV}$.

The number of events passing this selection criteria are referred to as the 'anti-electron' sample. The calorimeter isolation fraction in this 'anti-electron' sample contains contamination from electroweak backgrounds and also from real $\mathrm{CP} \mathrm{Z} \rightarrow e^{+} e^{-}$events. The distribution must therefore be corrected for these contaminations. The normalisation and shape of the contribution from $\mathrm{W}+$ jet events is predicted by applying the 'anti-electron' selection criteria to $\mathrm{W}+$ jet CdfSim. The signal contamination is also obtained using $\mathrm{CP} \mathrm{Z} \rightarrow e^{+} e^{-}$ CdfSim sample. The invariant mass distribution of the $\mathrm{Z}$ boson in 'anti-electron' events before correction for contamination is shown in Figure 8.18 and the absence of a peak at the $\mathrm{Z}$ mass shows that there is little contamination. The calorimeter isolation fraction of the 'anti-electron' sample before and after correcting for contamination is also shown in Figure 8.18.

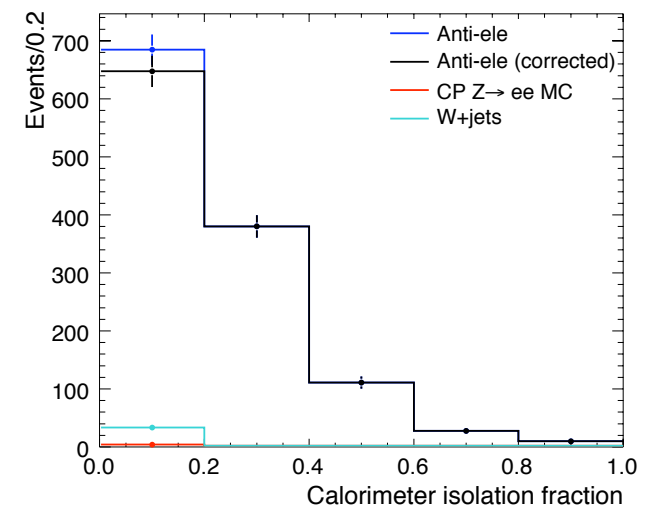

(a)

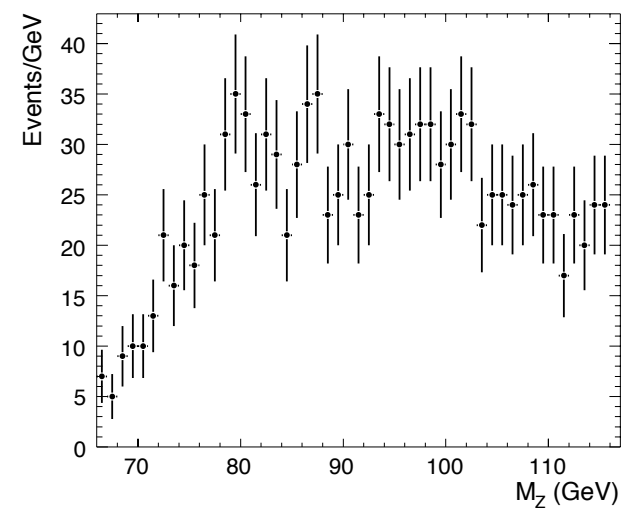

(b)

Figure 8.18: (a) The calorimeter isolation fraction distribution for the 'anti-electron' sample before and after correction for contamination from $\mathrm{W}+$ jet and signal $\mathrm{CP} \mathrm{Z} \rightarrow e^{+} e^{-}$events obtained from CdfSim. (b) The invariant mass of the Z boson in the 'anti-electron' sample. The absence of a peak at the Z mass shows that there is little signal contamination in the sample. 
The corrected isolation fraction distribution for the 'anti-electron' sample is used as the isolation shape of QCD background events in the $\mathrm{CP} \mathrm{Z} \rightarrow e^{+} e^{-}$sample. The signal shape of the isolation fraction is obtained from CdfSim CP Z $\rightarrow e^{+} e^{-}$events. The $\mathrm{W}+$ jet and $\mathrm{Z} \rightarrow \tau^{+} \tau^{-}$background shapes are added to the signal with the normalisations given in Section 8.6.1. The QCD background shape is also added to the signal shape and fits are performed to the data isolation distribution in the region above 0.4 whilst varying the amount of QCD background in the Monte Carlo histogram. The amount of QCD background that minimises the $\chi^{2}$ of the fit is found to be $\left(0.6 \pm 0.1_{\text {stat }}\right) \%$. The fit is shown in Figure 8.19.

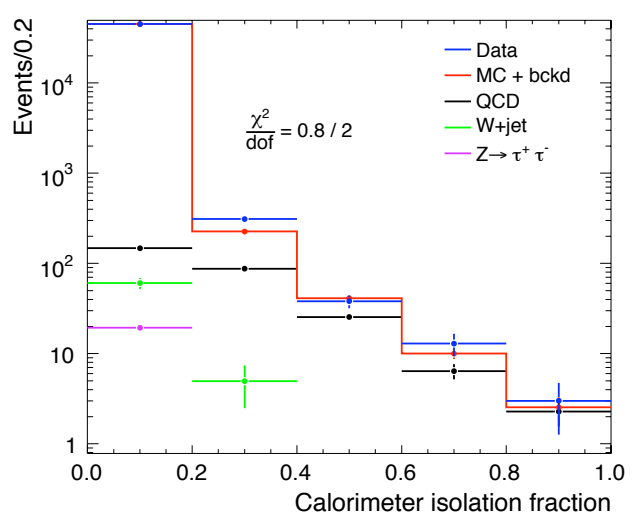

Figure 8.19: Fit to the calorimeter isolation fraction in the data to determine the fraction of QCD background in $\mathrm{CP} \mathrm{Z} \rightarrow e^{+} e^{-}$events.

A summary of the background processes and their relevant fractions contributing to $\mathrm{CP} \mathrm{Z} \rightarrow e^{+} e^{-}$events is shown in Table 8.4 , where the $\mathrm{Z} \rightarrow \tau^{+} \tau^{-}, \mathrm{W}+$ jet and QCD backgrounds have been calculated as described above and the diffractive background has been taken from [67]. The shape of the recoil distribution $(U)$ and its projection into directions parallel $\left(U_{1}\right)$ and perpendicular $\left(U_{2}\right)$ to the boson $p_{\mathrm{T}}$ is shown for background events in Figures $8.20,8.21$ and 8.22 . 


\begin{tabular}{|l|l|}
\hline Background process & Background fraction (\%) \\
\hline \hline $\mathrm{Z} \rightarrow \tau^{+} \tau^{-}$ & $0.040 \pm 0.001$ \\
$\mathrm{~W}+$ jet & $0.16 \pm 0.07$ \\
$\mathrm{QCD}$ & $0.6 \pm 0.1$ \\
diffractive & $1.0 \pm 0.2$ \\
\hline
\end{tabular}

Table 8.4: The background processes contributing to $\mathrm{CP} \mathrm{Z} \rightarrow e^{+} e^{-}$events and their relevant fractions.

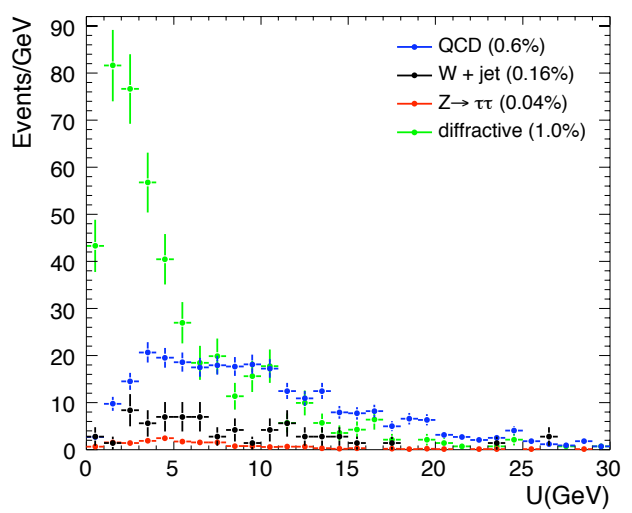

Figure 8.20: The shape of the recoil distribution in background events contributing to the CP sample.

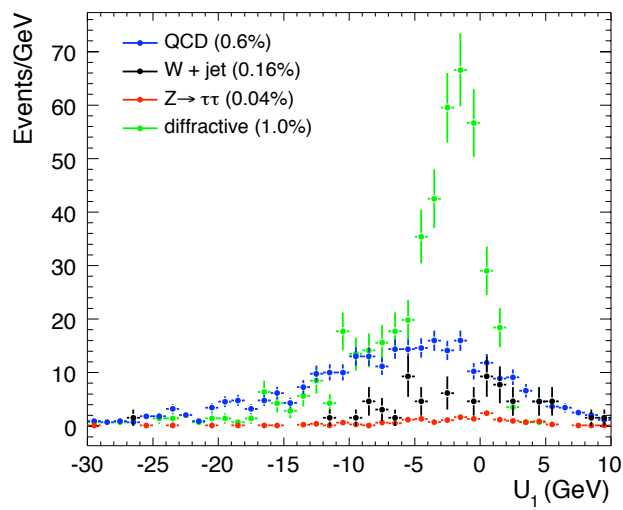

Figure 8.21: The shape of the $U_{1}$ distribution in background events contributing to the CP sample.

\subsection{Recoil Simulation}

The simulation of the recoil closely follows that described for $\mathrm{CC} \mathrm{Z} \rightarrow e^{+} e^{-}$events for the $\mathrm{W}$ mass analysis in the previous chapter. The recoil in the data receives contributions from initial state gluon radiation, underlying event energy and bremsstrahlung photons 


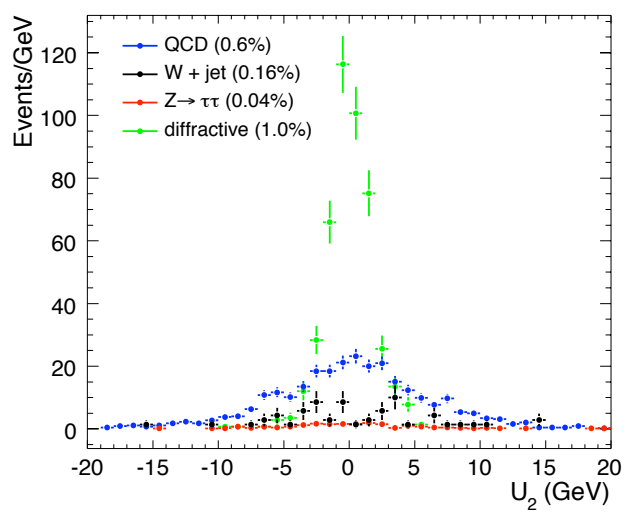

Figure 8.22: The shape of the $U_{2}$ distribution in background events contributing to the CP sample.

that are reconstructed outside of the knockout region. The bremsstrahlung contribution $\left(U_{B R E M}\right)$ is simulated by summing over the energy of photons propagated to towers in the central and plug calorimeter that lie outside of the knockout region for the central and plug electron. The $U_{Q C D}$ and $U_{U E}$ contributions are simulated by constructing a parametric model that is constrained by fits to the recoil in $\mathrm{CP} \mathrm{Z} \rightarrow e^{+} e^{-}$data. The recoil in $\mathrm{CP}$ events is parameterised using the same functional form as that for $\mathrm{CC} \mathrm{Z} \rightarrow l^{+} l^{-}$events for the $\mathrm{W}$ mass analysis. The recoil is projected onto two axes using the boson $p_{\mathrm{T}}$ direction. The projection of the recoil in the direction of the boson $p_{\mathrm{T}}\left(U_{1}\right)$ probes the contribution from initial state $\mathrm{QCD}$ radiation and the projection in the direction perpendicular to the boson $p_{\mathrm{T}}\left(U_{2}\right)$ is predominantly sensitive to the underlying energy contribution. The response of $U_{1}$ is parameterised as in Equation 7.12 with parameters $P_{1,2}$ constrained from a fit to the $\left\langle U_{1}\right\rangle$ vs. $p_{\mathrm{T}}$ distribution in data. The resolutions of $U_{1}$ and $U_{2}$ are parameterised as in Equations 7.13 and 7.14. The underlying energy is not expected to be different for $\mathrm{CP}$ events and the same parameters used to describe the resolution of underlying energy obtained from fits to minimum-bias are used. However, the $\Sigma E_{\mathrm{T}}$ distribution in CP events is different to $\mathrm{CC}$ events. The $\Sigma E_{\mathrm{T}}$ distribution in the data is therefore fitted using the 6 parameter functional form used to describe the $\Sigma E_{\mathrm{T}}$ in the $\mathrm{W}$ mass analysis. The functional form has a dependence on the boson $p_{\mathrm{T}}$ and luminosity. The total $\chi^{2}$ of four 
distributions; the inclusive $\Sigma E_{\mathrm{T}}$ distribution, $\left\langle\Sigma E_{\mathrm{T}}\right\rangle$ vs. luminosity, $\left\langle\Sigma E_{\mathrm{T}}\right\rangle$ vs. boson $p_{\mathrm{T}}, \sigma\left(\Sigma E_{\mathrm{T}}\right)$ vs. luminosity and $\sigma\left(\Sigma E_{\mathrm{T}}\right)$ vs. $p_{\mathrm{T}}$ is minimised to obtain the best fit $\Sigma E_{\mathrm{T}}$ parameters. The fit results are shown in Figure 8.23 and Figure 8.24. As mentioned in Chapter 7, perfect agreement between data and simulation for these distributions is not vital since $\Sigma E_{\mathrm{T}}$ enters into the definition of $\sigma_{M B}$ which enters as part of $\sigma\left(U_{1}\right), \sigma\left(U_{2}\right)$. The resolutions of $U_{1}$ and $U_{2}$ are fit for so any minor mismodelling in the $\Sigma E_{\mathrm{T}}$ description is corrected in the $\sigma\left(U_{1}\right)$ and $\sigma\left(U_{2}\right)$ modelling and as shown in Chapter 7 they have a negligible effect on the transverse mass distribution and hence the fitted mass of the W boson.

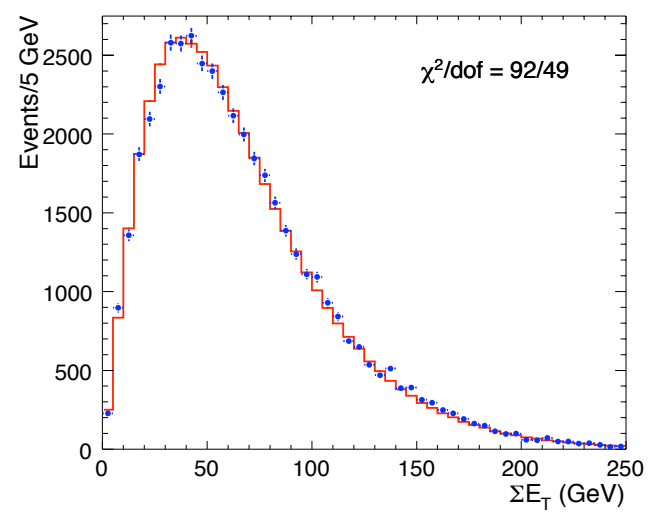

Figure 8.23: $\Sigma E_{\mathrm{T}}$ distribution in $\mathrm{CP} \mathrm{Z} \rightarrow e^{+} e^{-}$events compared to the simulation using the best fit $\Sigma E_{\mathrm{T}}$ parameters.

The fit to the variation of the $U_{1}$ response with boson $p_{\mathrm{T}}$ to obtain $P_{1,2}$ is shown in Figure 8.25. Parameters $P_{3,4}$ are obtained from a fit to the $\sigma\left(U_{1}\right)$ vs. $p_{\mathrm{T}}$ distribution, shown in Figure 8.26 and $P_{5,6}$ are constrained from a fit to the $\sigma\left(U_{2}\right)$ vs. $p_{\mathrm{T}}$ distribution, shown in Figure 8.27. The best fit recoil parameters are given in Table 8.5. The inclusive $U_{1}, U_{2}$ and $U$ distributions are shown in Figure 8.28 for data and simulation. The dominant background contributions to the $\mathrm{CP} \mathrm{Z} \rightarrow e^{+} e^{-}$sample are from diffractive events and QCD events and these backgrounds are included in the simulation recoil distributions. 


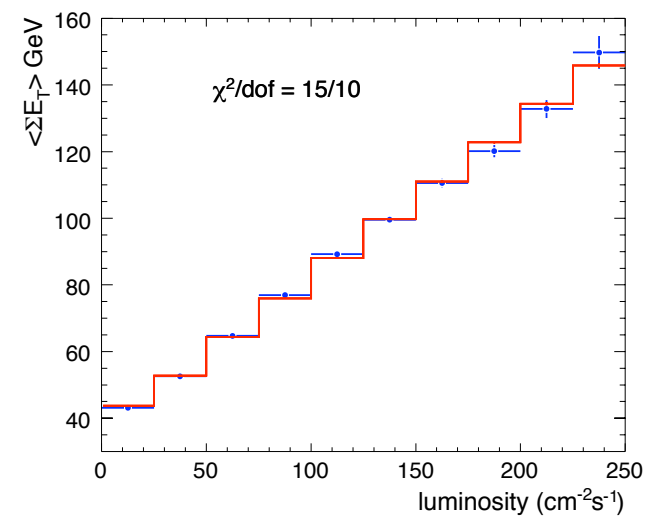

(a)

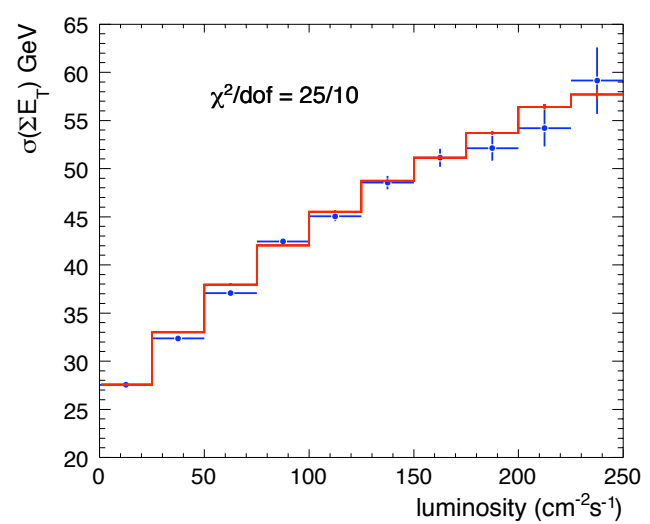

(c)

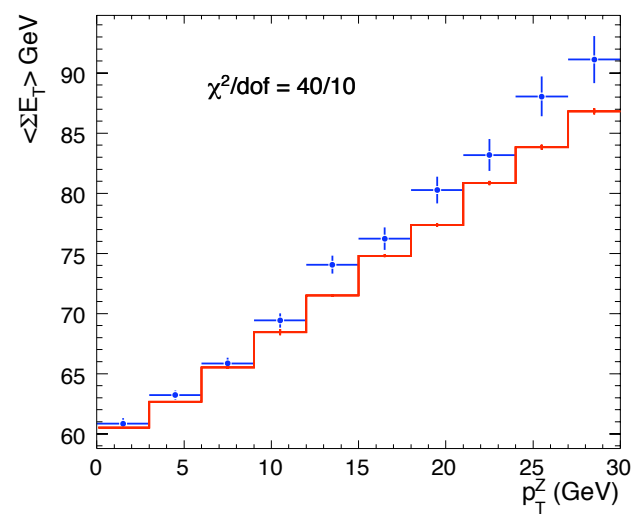

(b)

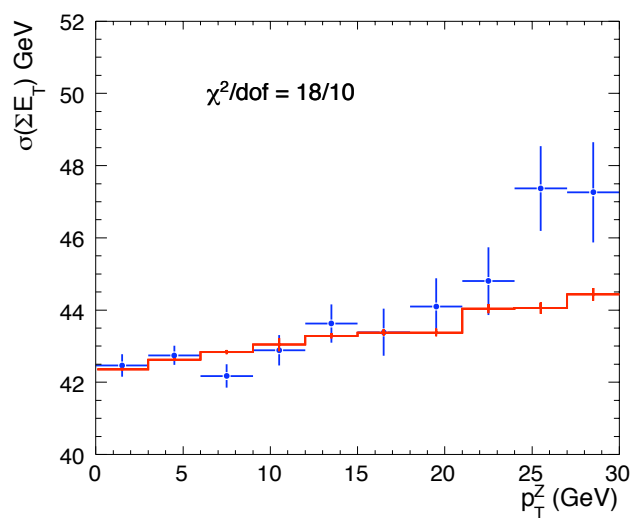

(d)

Figure 8.24: The dependence of the mean and standard deviation of the $\Sigma E_{\mathrm{T}}$ on luminosity and boson $p_{\mathrm{T}}$ in $\mathrm{CP} \mathrm{Z} \rightarrow e^{+} e^{-}$events. These distributions are used in the fit to obtain the $\Sigma E_{\mathrm{T}}$ parameters.

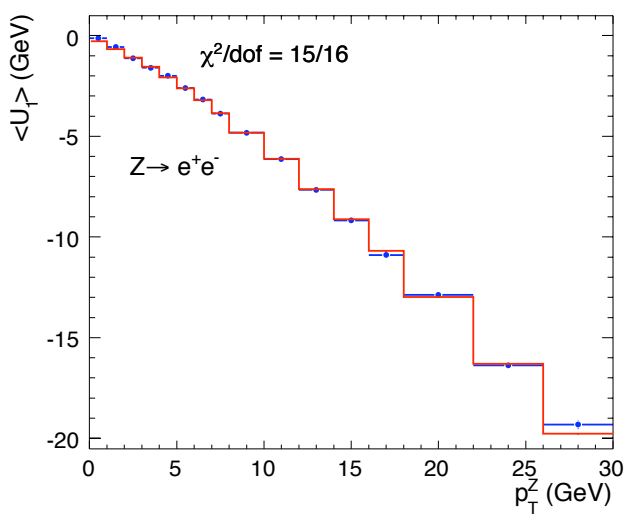

Figure 8.25: The variation of the $U_{1}$ response with boson $p_{\mathrm{T}}$ in $\mathrm{CP} \mathrm{Z} \rightarrow e^{+} e^{-}$events. 


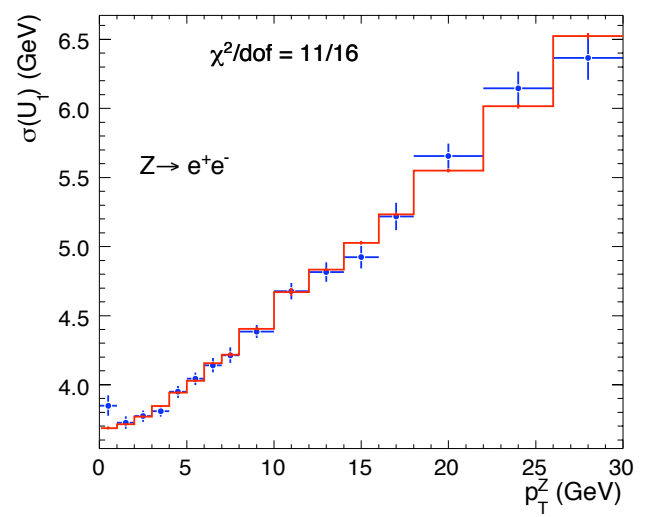

Figure 8.26: The variation of the resolution of $U_{1}$ with boson $p_{\mathrm{T}}$ in $\mathrm{CP} \mathrm{Z} \rightarrow e^{+} e^{-}$events.

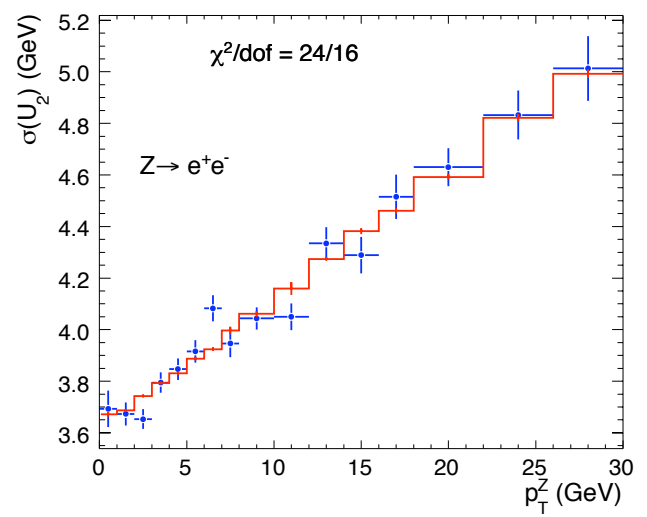

Figure 8.27: The variation of the resolution of $U_{2}$ with boson $p_{\mathrm{T}}$ in $\mathrm{CP} \mathrm{Z} \rightarrow e^{+} e^{-}$events.

\subsection{Results}

The recoil parameters obtained from fits to CP and CC data are shown in Table 8.5. The decorrelated response parameters $P_{1}$ and $P_{2}$ in CP events can be compared with those obtained from fits to CC data. It shows that the response parameters are consistent within their statistical uncertainties. Since the $U_{1}$ and $U_{2}$ resolution parameters are highly correlated, parameters $P_{3}$ to $P_{6}$ cannot be compared directly. However, using the $\mathrm{CC}$ recoil parameters for $\mathrm{CP}$ events increases the total $\chi^{2} /$ dof of the three fitted $\mathrm{Z}$ distributions from $46 / 48$ to $126 / 48$.

It is important therefore to estimate the effect of the difference between the CC and 


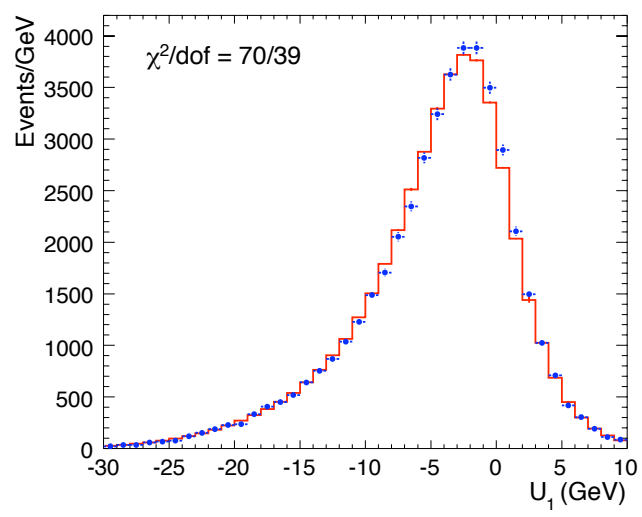

(a)

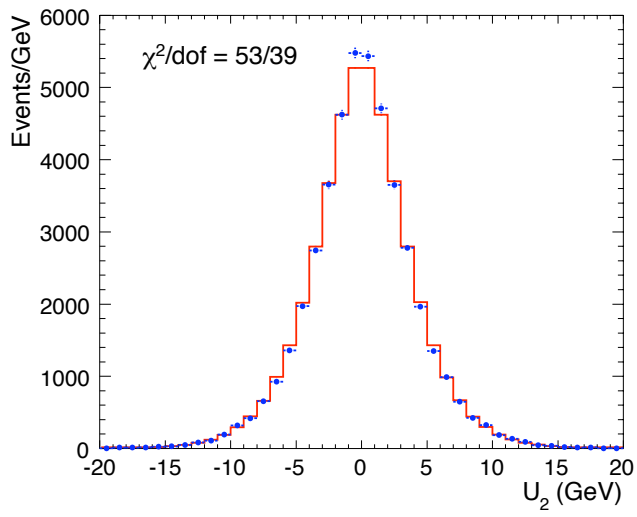

(b)

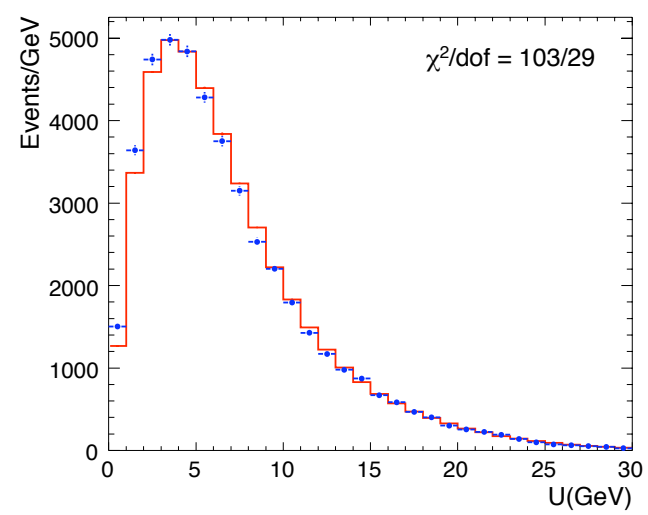

(c)

Figure 8.28: Recoil distributions of (a) $U_{1}$, (b) $U_{2}$ and (c) $U$ in $\mathrm{CP} \mathrm{Z} \rightarrow e^{+} e^{-}$events compared to the best fit simulation.

CP parameters on the $M_{T}$ distribution and hence the fitted $\mathrm{W}$ mass. This is studied by fitting to the $M_{T}$ distribution in $\mathrm{W} \rightarrow e \nu$ events produced using $\mathrm{CP}$ recoil parameters using templates of the $M_{T}$ distribution that have been created using the CC parameters. This gives a shift in the value of the fitted $\mathrm{W}$ mass from using $\mathrm{CP}$ parameters instead of CC parameters. A shift of $19 \mathrm{MeV}$ in the value of the $\mathrm{W}$ mass is obtained. Since $\mathrm{W}$ events are a mixture of approximately $70 \% \mathrm{CC}$ events and $30 \% \mathrm{CP}$ events, the systematic on the $\mathrm{W}$ mass from using recoil parameters tuned only on $\mathrm{CC}$ events is $6 \mathrm{MeV}$. 


\begin{tabular}{|l|c|c|}
\hline Parameter & CP & CC \\
\hline \hline$P_{1}$ & $0.623 \pm 0.003$ & $0.625 \pm 0.003$ \\
$P_{2}$ & $10.2 \pm 0.8$ & $9.5 \pm 0.9$ \\
$P_{3}$ & $0.941 \pm 0.008$ & $0.971 \pm 0.009$ \\
$P_{4}$ & $0.0145 \pm 0.0008$ & $0.0105 \pm 0.0009$ \\
$P_{5}$ & $0.971 \pm 0.008$ & $1.023 \pm 0.009$ \\
$P_{6}$ & $0.0029 \pm 0.0007$ & $-0.0023 \pm 0.0008$ \\
\hline
\end{tabular}

Table 8.5: The recoil parameters obtained from fits to the recoil in $\mathrm{CP}$ and $\mathrm{CC} \mathrm{Z} \rightarrow e^{+} e^{-}$events.

\subsection{Summary}

The recoil parameterisation for $\mathrm{W}$ events is obtained by fitting to the recoil in $\mathrm{Z} \rightarrow l^{+} l^{-}$ events which have a different event topology to $\mathrm{W} \rightarrow l \nu$ events since both leptons in $\mathrm{Z} \rightarrow l^{+} l^{-}$events are reconstructed in the central region whereas no such requirement can be made on the neutrino in $\mathrm{W} \rightarrow l \nu$ events. The Central-Plug analysis involves studying the recoil in events where one electron is reconstructed in the plug calorimeter and the other in the central calorimeter. The simulation of $\mathrm{CP}$ events involves determining the PEM scale and resolution by fitting to the $\mathrm{Z}$ invariant mass distribution, calculating the efficiency of the selection cuts, simulating the knockout region for the central and plug electron and calculating the backgrounds that could affect the recoil distributions. The recoil fits are subsequently performed to obtain the recoil parameters that best describe the recoil in $\mathrm{CP}$ events. These parameters are used to produce the transverse mass distribution which is fitted using templates produced with CC parameters to obtain a shift in the value of the fitted $\mathrm{W}$ mass. A systematic of $6 \mathrm{MeV}$ on the $\mathrm{W}$ mass is obtained from using recoil parameters for $\mathrm{W}$ events that have only been tuned on $\mathrm{CC}$ events. 


\section{Chapter 9}

\section{Backgrounds}

The $\mathrm{W} \rightarrow l \nu$ events passing the selection criteria described in Chapter 4 are high purity samples with small contaminations from backgrounds. The background events have a different $M_{T}$ distribution to real $\mathrm{W} \rightarrow l \nu$ events and can bias the fitted mass and width of the $\mathrm{W}$ boson. The background shapes and normalisations are therefore determined and added to the simulation templates of the $M_{T}$ distributions when fitting for these quantities.

In the $\mathrm{W}$ width analysis, only the backgrounds in $\mathrm{W} \rightarrow l \nu$ events are considered since there is negligible background in the $\mathrm{Z} \rightarrow l^{+} l^{-}$samples. However, in the $\mathrm{W}$ mass analysis, the background in $\mathrm{Z} \rightarrow l^{+} l^{-}$events are also estimated.

The backgrounds contributing to the data samples can be divided into two categories, electroweak backgrounds which include well understood processes like $\mathrm{W} \rightarrow \tau \nu$, $\mathrm{Z} \rightarrow l^{+} l^{-}$and $\mathrm{Z} \rightarrow \tau^{+} \tau^{-}$and are estimated using a Monte Carlo based approach and non-electroweak backgrounds with contributions from QCD events which are extracted from data. The $\mathrm{W} \rightarrow \mu \nu$ channel also receives non-electroweak background contribution from long lived hadrons that decay in the tracker volume to $\mu \nu$ pairs, known as decay in-flight events.

This chapter will describe the methods used to estimate the backgrounds in the W mass analysis, focusing on the electron channel. The backgrounds in the electron and muon channels for the $\mathrm{W}$ width analysis are discussed in detail in [61] and the systematic on $\Gamma_{W}$ from the amount and shape of the backgrounds is also given. 


\subsection{Electroweak Backgrounds}

The normalisation and shape of the electroweak backgrounds contributing to $\mathrm{Z} \rightarrow e^{+} e^{-}$ and $\mathrm{W} \rightarrow e \nu$ events can be estimated using large Monte Carlo samples that are generated using PYTHIA and simulated using CdfSim. The electroweak backgrounds contributing to $\mathrm{W} \rightarrow e \nu$ events are $\mathrm{Z} \rightarrow e^{+} e^{-}, \mathrm{Z} \rightarrow \tau^{+} \tau^{-}$and $\mathrm{W} \rightarrow \tau \nu$ and the dominant background contributing to $\mathrm{Z} \rightarrow e^{+} e^{-}$events is $\mathrm{Z} \rightarrow \tau^{+} \tau^{-}$.

\subsubsection{Electroweak Backgrounds in $\mathrm{W} \rightarrow e \nu$}

\section{$\mathrm{W} \rightarrow \tau \nu$ background}

$\mathrm{W} \rightarrow \tau \nu$ events where the $\tau$ decays to an electron and a neutrino can fake the electron and missing energy signature of real $\mathrm{W} \rightarrow e \nu$ events. Since this is a four-body decay, the charged lepton carries a significantly lower transverse momentum and the transverse mass distribution of such events is skewed towards lower values. Whereas these events are kinematically suppressed because of the high $E_{T}$ requirement on the electron in $\mathrm{W}$ decay, they form the dominant background in the electron channel.

The fraction of $\mathrm{W} \rightarrow \tau \nu$ events in the $\mathrm{W} \rightarrow e \nu$ candidate sample is determined by propagating CdfSim samples of $\mathrm{W} \rightarrow \tau \nu$ and $\mathrm{W} \rightarrow e \nu$ events through the event selection. The proportion of these events passing the event selection compared to the total number of events generated gives their respective acceptances. Assuming lepton universality, the cross-section times branching ratio for $\mathrm{W} \rightarrow e \nu$ and $\mathrm{W} \rightarrow \tau \nu$ events is the same and so the amount of $\mathrm{W} \rightarrow \tau \nu$ background can be estimated by the ratio of acceptance of $\mathrm{W} \rightarrow \tau \nu$ to $\mathrm{W} \rightarrow e \nu$ events. The background is found to be $\left(0.95 \pm 0.01_{\text {stat. }}\right) \%$ of the $\mathrm{W} \rightarrow e \nu$ sample. $\mathrm{Z} / \gamma^{*} \rightarrow l^{+} l^{-}$background

$\mathrm{W} \rightarrow e \nu$ events can also be contaminated by $\mathrm{Z} / \gamma^{*} \rightarrow l^{+} l^{-}$background, where $l=e, \tau$. $\mathrm{Z} / \gamma^{*} \rightarrow l^{+} l^{-}$events can mimic the $\mathrm{W} \rightarrow e \nu$ signature if one of the leptons falls into an 
uninstrumented region of the detector and is not reconstructed or is significantly mismeasured such that it fakes missing energy. The $\mathrm{Z} / \gamma^{*} \rightarrow l^{+} l^{-}$background fraction is estimated by multiplying the ratio of acceptance of $\mathrm{Z} / \gamma^{*} \rightarrow l^{+} l^{-}$to $\mathrm{W} \rightarrow e \nu$ events by the ratio of their corresponding cross-section times branching ratios. The $\mathrm{Z} / \gamma^{*} \rightarrow l^{+} l^{-}$events passing the event selection are most likely to be pure $\mathrm{Z} \rightarrow l^{+} l^{-}$events and therefore the NNLO calculation of the ratio of $\mathrm{W} \rightarrow l \nu$ to $\mathrm{Z} \rightarrow l^{+} l^{-}$cross-section; $R=\sigma(\mathrm{W} \rightarrow l \nu) / \sigma\left(\mathrm{Z} \rightarrow l^{+} l^{-}\right)=$ $10.67 \pm 0.15$ [63], can be used. The uncertainty on this ratio $R$ contributes as an uncertainty on the measured background. The $\mathrm{Z} / \gamma^{*} \rightarrow e^{+} e^{-}$and $\mathrm{Z} / \gamma^{*} \rightarrow \tau^{+} \tau^{-}$backgrounds are estimated to contribute $\left(0.268 \pm 0.001_{\text {stat. }} \pm 0.004_{R}\right) \%$ and $\left(0.065 \pm 0.001_{\text {stat. }} \pm 0.001_{R}\right) \%$ respectively to the electron channel.

The shape of the $M_{T}$ distribution of the electroweak backgrounds contributing to $\mathrm{W} \rightarrow e \nu$ events is compared in Figure 9.1.

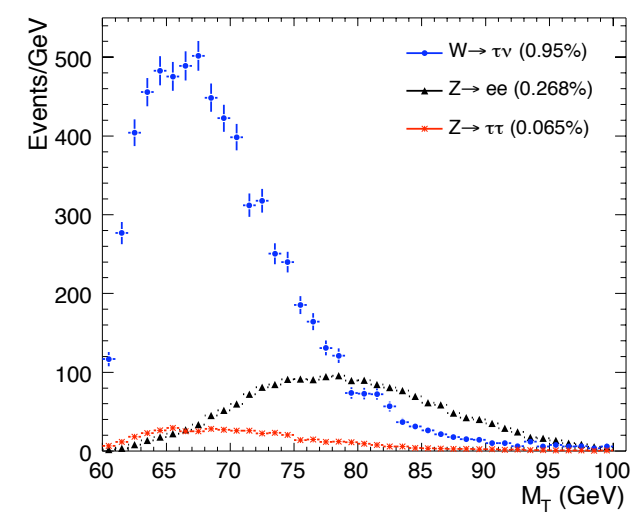

Figure 9.1: Comparison between the shape of the $M_{T}$ distribution in $\mathrm{W} \rightarrow \tau \nu, \mathrm{Z} \rightarrow e^{+} e^{-}$and $\mathrm{Z} \rightarrow \tau^{+} \tau^{-}$ background events in the $\mathrm{W} \rightarrow e \nu$ candidate sample.

\subsubsection{Electroweak Background in $\mathrm{Z} \rightarrow e^{+} e^{-}$}

\section{$\mathrm{Z} \rightarrow \tau^{+} \tau^{-}$background}

$\mathrm{Z} \rightarrow \tau^{+} \tau^{-}$events where both of the $\tau$ leptons decay into an $e \nu$ pair can mimic the $\mathrm{Z} \rightarrow e^{+} e^{-}$signature. The background is found by propagating a CdfSim sample of $\mathrm{Z} \rightarrow \tau^{+} \tau^{-}$ events through the event selection. The same is done for signal CdfSim events and the 
ratio of acceptances of $\mathrm{Z} \rightarrow \tau^{+} \tau^{-}$to $\mathrm{Z} \rightarrow e^{+} e^{-}$events is multiplied by the ratio of their corresponding cross-sections times branching ratio. The amount of $\mathrm{Z} \rightarrow \tau^{+} \tau^{-}$background is found to be $\left(0.041 \pm 0.004_{\text {stat. }}\right) \%$.

\subsection{Diffractive Background}

In addition to electroweak and QCD backgrounds, diffractive events can also contaminate the $\mathrm{Z} \rightarrow e^{+} e^{-}$and $\mathrm{W} \rightarrow e \nu$ samples. Diffractive events are $p \bar{p}$ collisions in which the interacting proton or antiproton remains intact. A characteristic of these events is a large phase space region that is devoid of particles, known as a rapidity gap. A real $\mathrm{W}$ or $\mathrm{Z}$ boson can be produced in diffractive events, at LO from a quark interaction in the pomeron (a colour singlet combination of gluons and/or quarks with the quantum numbers of the vacuum) and at NLO via a gluon which can be distinguished from the former process by the presence of an additional jet in the final state. The production diagrams for these processes are shown in Figure 9.2. These events comprise approximately $1 \%$ of the total $\mathrm{W} / \mathrm{Z}$ production [67]. The shape of these events contributing to the $\mathrm{W}$ and $\mathrm{Z}$ dataset is obtained from a CdfSim sample of diffractive events which are passed through the full $\mathrm{Z} \rightarrow e^{+} e^{-}$and $\mathrm{W} \rightarrow e \nu$ selection criteria to determine the shape.

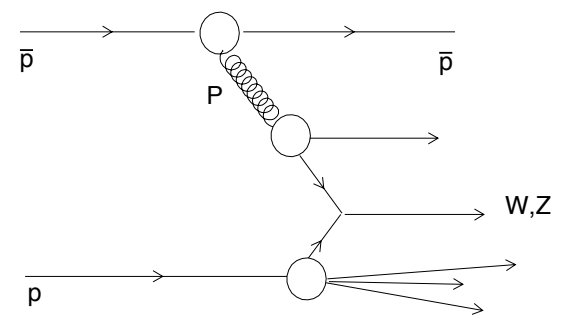

(a)

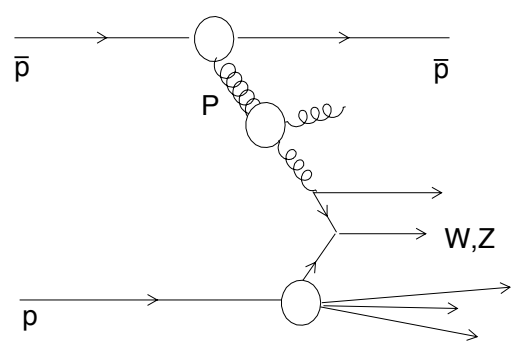

(b)

Figure 9.2: The diffractive production of a $\mathrm{W}$ boson at (a) LO from a quark interaction in the pomeron (P) and (b) at NLO from a gluon. 


\subsection{QCD Background}

\section{Background in $\mathrm{Z} \rightarrow e^{+} e^{-}$}

QCD events in which jets (or photons) fake both electrons can pass the $\mathrm{Z} \rightarrow e^{+} e^{-}$selection criteria. QCD background events have a very different shape to real $\mathrm{Z} \rightarrow e^{+} e^{-}$events and it is instructive to evaluate how this could affect the calibrations of the detector that are tuned on $\mathrm{Z} \rightarrow e^{+} e^{-}$samples, with particular emphasis on the parameterisation of the recoil.

In general, two methods are employed to estimate the level of QCD background in $\mathrm{Z} \rightarrow e^{+} e^{-}$events, one method is to study the proportion of events with the same sign charge for both charged leptons and the other is to perform a fit to the isolation distribution.

It is assumed that QCD events are charge symmetric, i.e there are equal numbers of events where the two fake electrons have the same sign charge (same-sign events) and where they have opposite sign charge (opposite-sign events). The number of QCD events can be estimated from the number of same-sign events in the $\mathrm{Z} \rightarrow e^{+} e^{-}$sample since it will be assumed that there is an equal number of opposite-sign events that passed the selection requirements. The ratio of same-sign events to opposite sign events in $\mathrm{Z} \rightarrow e^{+} e^{-}$ data is $(1.24 \pm 0.06) \%$. However, some real $\mathrm{Z} \rightarrow e^{+} e^{-}$events may also be same-sign if one of the electrons radiates an energetic photon that subsequently converts and the conversion electron with the opposite sign charge to the original electron is associated with the electromagnetic cluster in the calorimeter. The invariant mass distribution of $\mathrm{Z} \rightarrow e^{+} e^{-}$ events that have passed all the selection criteria except the opposite sign requirement is shown in Figure 9.3. An obvious peak at the mass of the $\mathrm{Z}$ boson is observed in the distribution suggesting that not all of these are QCD events and there is significant contamination from real $\mathrm{Z} \rightarrow e^{+} e^{-}$conversion events. The fraction of same-sign events from conversions that can be expected in the $\mathrm{Z} \rightarrow e^{+} e^{-}$sample can be obtained using both 
CdfSim and the fast simulation. The percentage of same-sign to opposite-sign events predicted by CdfSim is $(0.84 \pm 0.01) \%$ and by the fast simulation is $0.6 \%$. The amount of QCD background predicted by the same-sign method is taken as the difference between the value given by CdfSim and the data to give $(0.4 \pm 0.2) \%$. The uncertainty on this number is large and covers both predictions from CdfSim and the fast simulation.

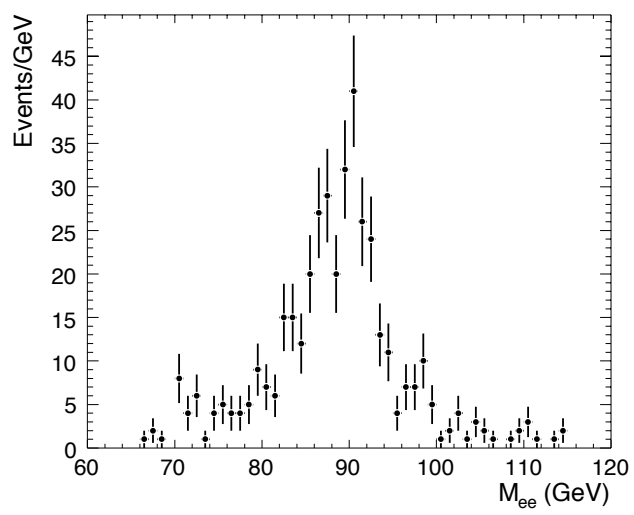

Figure 9.3: The invariant mass distribution in $\mathrm{Z} \rightarrow e^{+} e^{-}$sample for electrons with same sign charge.

The other method employed to estimate the amount of QCD background in $\mathrm{Z} \rightarrow e^{+} e^{-}$ events is using the calorimeter isolation variable. The jets faking the two electrons in the event are generally accompanied by hadronic activity so the energy in the calorimeter is not as isolated as it would be for a real electron. A sample rich in QCD events is selected from the data by imposing all the default cuts with some cuts relaxed as described in the following:

- $\chi_{\text {strip }}^{2}$ cut.

- $E_{\text {had }} / E_{\text {em }}$ cut to $E_{\text {had }} / E_{\text {em }}<0.125$.

- $E / p$ cut to $E / p<2.5$.

- $\left|\Delta z_{0}\right|$ cut to $\left|\Delta z_{0}\right|<8 \mathrm{~cm}$.

- At least two of the following 'anti-electron cuts' are required to pass: 


$$
\begin{aligned}
& -E / p>2.3 \\
& -L_{\mathrm{shr}}>0.2 \\
& -\chi_{\text {strip }}^{2}>10.0 \\
& -E_{\mathrm{had}} / E_{\mathrm{em}}>0.1 \\
& -\left|\Delta z_{0}\right|>5 \mathrm{~cm}
\end{aligned}
$$

The 'anti-electron' sample is dominated by QCD events but also contains contaminations from other processes, where the dominant one is signal events. The amount of signal contamination in the 'anti-electron' sample is predicted by using CdfSim events and propagating them through the 'anti-electron' event selection. It is assumed that CdfSim correctly predicts the level of signal contamination in the 'anti-electron' sample. The 'anti-electron' calorimeter isolation ratio distribution is shown before and after correcting for contamination in Figure 9.4(a). The invariant mass distribution of the $\mathrm{Z}$ boson is also shown before and after correcting for contamination in Figure 9.4(b). There is an obvious peak at the $\mathrm{Z}$ mass before the correction.

The corrected sample of events is then considered to be pure QCD events. The calorimeter isolation ratio for signal $\mathrm{Z} \rightarrow e^{+} e^{-}$is obtained from CdfSim. The distributions from $\mathrm{Z} \rightarrow \tau^{+} \tau^{-}$and diffractive events are also obtained from CdfSim and added to the signal distribution. The QCD isolation distribution is then added to this and the $\chi^{2}$ of the fit to data is minimised whilst varying the normalisation of the QCD background. The fit is shown in Figure 9.5(a) and the variation of the fit $\chi^{2}$ around the minima is shown in Figure 9.5(b). The QCD background is found to be $\left(0.25 \pm 0.10_{\text {stat. }}\right) \%$.

The estimates of QCD background obtained from the two methods are consistent within their uncertainties. The background value of $\left(0.25 \pm 0.10_{\text {stat. }} \pm 0.15_{\text {sys. }}\right) \%$ is used, where the systematic uncertainty is chosen to cover the results from both methods. 


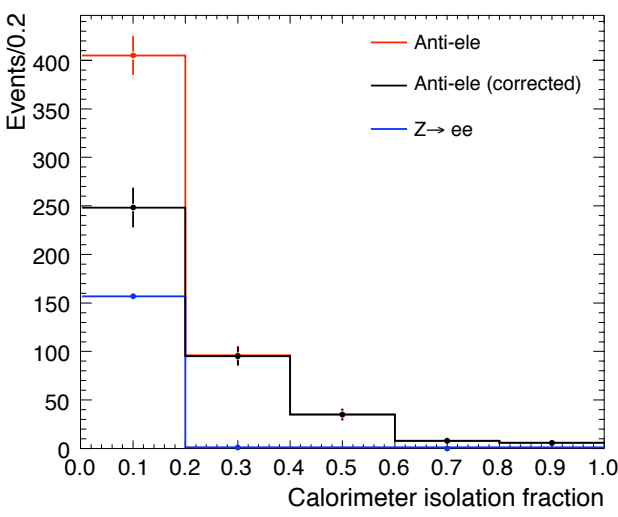

(a)

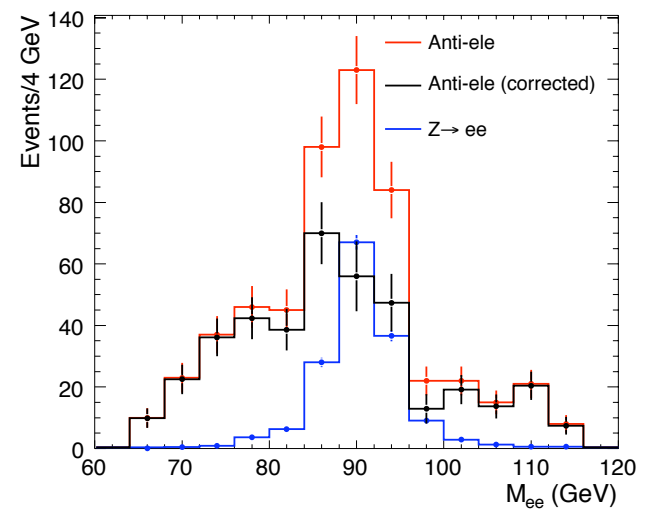

(b)

Figure 9.4: (a) The calorimeter isolation fraction distribution in the 'anti-electron' sample before (shown in red) and after (shown in black) correcting for electroweak contamination. (b) The invariant mass of the $\mathrm{Z}$ boson before and after correcting for electroweak contamination.

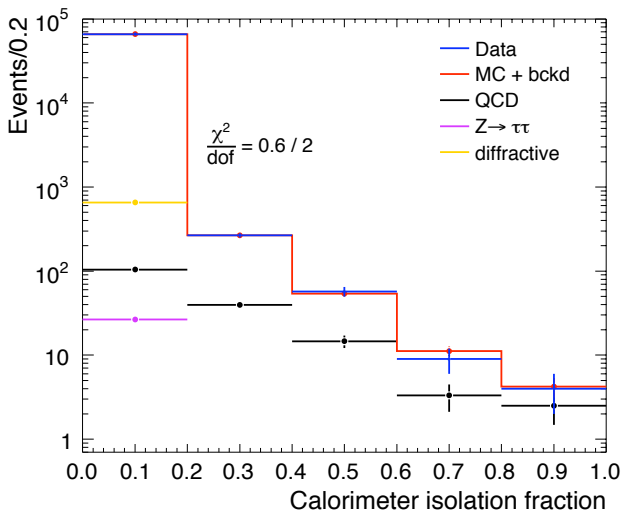

(a)

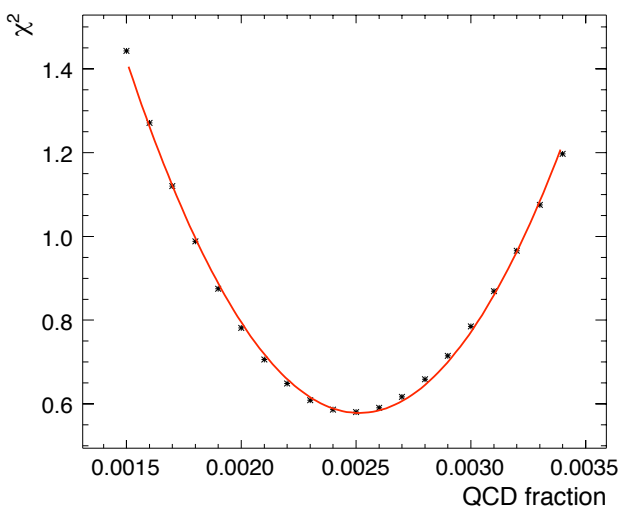

(b)

Figure 9.5: (a) Result of the fit to the calorimeter isolation fraction distribution to obtain the normalisation of QCD background. (b) The variation of the $\chi^{2}$ of the fit as a function of the amount of QCD background.

\section{Background in $\mathrm{W} \rightarrow e \nu$}

QCD events in which a jet fakes an electron and the energy of another jet is significantly mismeasured to produce missing energy can mimic the signature of a $\mathrm{W} \rightarrow e \nu$ event. QCD background in $\mathrm{W} \rightarrow e \nu$ events is significantly suppressed by requiring high transverse momentum for the charged lepton, large missing energy and low recoil energy in the event.

The amount of QCD background in $\mathrm{W} \rightarrow e \nu$ events is estimated by fitting to the 
$E_{T}$ distribution. Since the missing energy in QCD events is not real and the result of a significantly undermeasured jet energy, these events populate the low $\notin_{T}$ region with a distribution different to that in $\mathrm{W} \rightarrow e \nu$ events. The $\mathbb{E}_{T}$ variable therefore has some discriminating power to distinguish between $\mathrm{QCD}$ events and real $\mathrm{W} \rightarrow e \nu$ events.

The shape of the $\mathbb{E}_{T}$ distribution in QCD events is extracted from the data by removing the $\mathbb{H}_{T}$ and $M_{T}$ cuts and requiring the electron to pass certain 'anti-electron' cuts to select a subsample that is predominantly QCD events. In general, the amount of QCD background predicted using this method has some dependence on the 'anti-electron' selection criteria. Two sets of selection criteria are defined, a default selection and a 'tight' selection, where tighter cuts are made on all of the 'anti-electron' cuts.

The following cuts are relaxed with only the upper limits described earlier in this section: $\chi_{\text {strip }}^{2}, E_{\text {had }} / E_{\text {em }}, \Delta z_{0}, E / p$ and $L_{\text {shr }}$. The values of the 'anti-electron' cuts in the default selection are as follows:

- $Q \Delta x<-3.0, Q \Delta x>1.5$

- $0.2<L_{\mathrm{shr}}<1.0$

- $\chi_{\text {strip }}^{2}>20.0$

- $E_{\text {had }} / E_{\text {em }}>0.08$

where the variable $Q \Delta x$ is simply the charge $(Q)$ of the electron multiplied by $\Delta x$. The values of the 'anti-electron' cuts in the 'tight' selection are:

- $Q \Delta x<-4.0, Q \Delta x>2.0$

- $0.35<L_{\mathrm{shr}}<1.0$

- $\chi_{\text {strip }}^{2}>30.0$

- $E_{\text {had }} / E_{\text {em }}>0.09$. 
A set of selection criteria are obtained by using different combinations of the cuts from the default and 'tight' selections listed above. Each of the selection criteria are applied to $\mathrm{W} \rightarrow e \nu$ data events to produce subsamples that are dominated by QCD events but also contain contaminations from real $\mathrm{W} \rightarrow e \nu$ events and other electroweak backgrounds that also passed the selection criteria. The $\not_{T}$ distribution in each sample must therefore be corrected to account for these contaminations. There are contributions to these samples from $\mathrm{W} \rightarrow e \nu, \mathrm{Z} \rightarrow e^{+} e^{-}, \mathrm{W} \rightarrow \tau \nu$ and $\mathrm{Z} \rightarrow \tau^{+} \tau^{-}$processes and the shape and normalisation of their contribution is obtained using CdfSim. The 'anti-electron' $\not_{T}$ distribution for some of the selection criteria is shown before and after correcting for contamination in Figure 9.6.

The QCD rich sample obtained after this correction is used as the shape of the background in $\mathrm{W} \rightarrow e \nu$ events. The distribution is added to the $\mathbb{F}_{T}$ distribution for signal events which is obtained from the fast simulation and also to other electroweak backgrounds and a fit to the $\not_{T}$ distribution is performed whilst varying the normalisation of QCD background. This procedure is repeated for each selection criteria and the amount of QCD background obtained is plotted in Figure 9.7. It shows that different selection criteria give a wide range of QCD background estimates. However, it can be seen that for some of these event selections, the $\mathrm{QCD} \not_{T}$ distribution has some remaining contamination from signal or other backgrounds after correction. This is particularly evident in Figure 9.6(c), obtained by requiring at least 2 or more of the default 'anti-electron' cuts to pass, which corresponds to point 7 in Figure 9.7 and gives the highest QCD background estimate of $1.3 \%$. The $E_{T}$ distribution is very contaminated before the correction, with CdfSim predicting that $40 \%$ of the events in the signal region $\left(30<E_{T}<55 \mathrm{GeV}\right)$ are true $\mathrm{W} \rightarrow e \nu$ events or electroweak backgrounds. It can be seen that there is a remaining feature in the signal region even after signal and other backgrounds have been subtracted from the distribution. This estimate of the QCD background is therefore not considered to be reliable. 
The three $E_{T}$ distributions shown in Figures 9.6(a), 9.6(d) and 9.6(e) do not show such obvious features in the signal region. These QCD $\#_{T}$ distributions yield background estimates of $(0.577 \pm 0.036) \%,(0.541 \pm 0.031) \%$ and $(0.682 \pm 0.026) \%$ respectively. The QCD background value of $\left(0.682 \pm 0.026_{\text {stat. }} \pm 0.140_{\text {sys. }}\right) \%$ is chosen since this sample provides sufficient statistics in the signal region to obtain shapes for the $\nexists_{T}$ distribution, the $M_{T}$ distribution and also the recoil distributions. The systematic uncertainty on this value is chosen to cover the background estimates obtained from the other two 'anti-electron' selection criteria. The fit to the $E_{T}$ distribution that yields this background estimate is shown in Figure 9.8. 


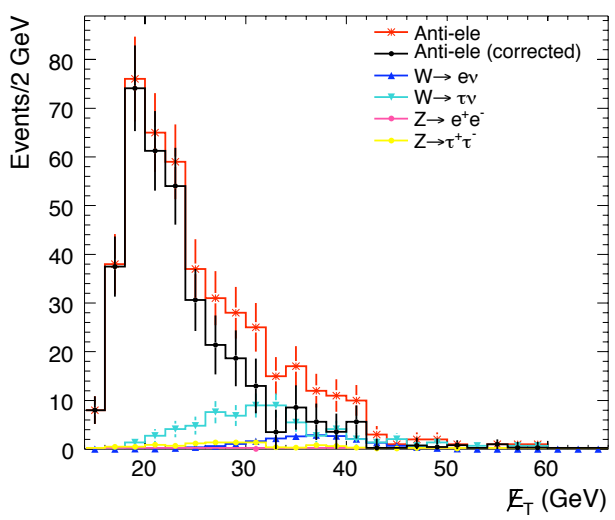

(a)

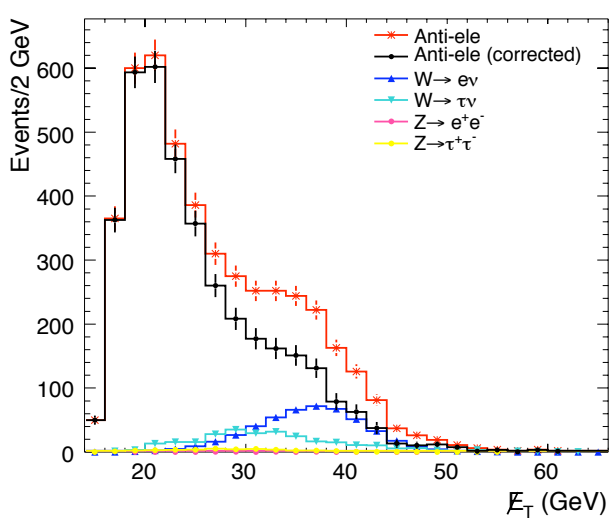

(c)

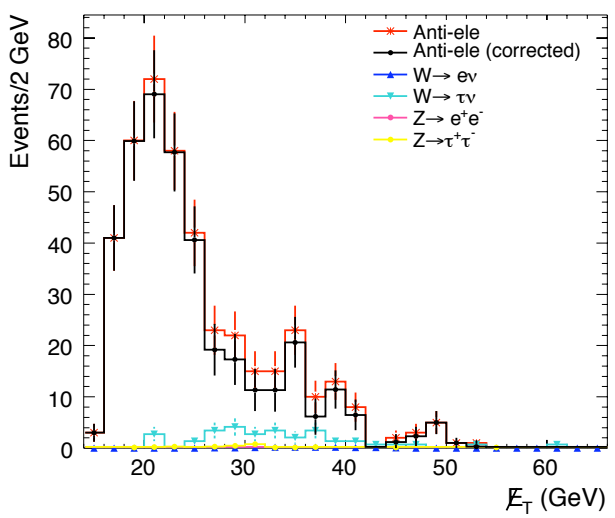

(b)

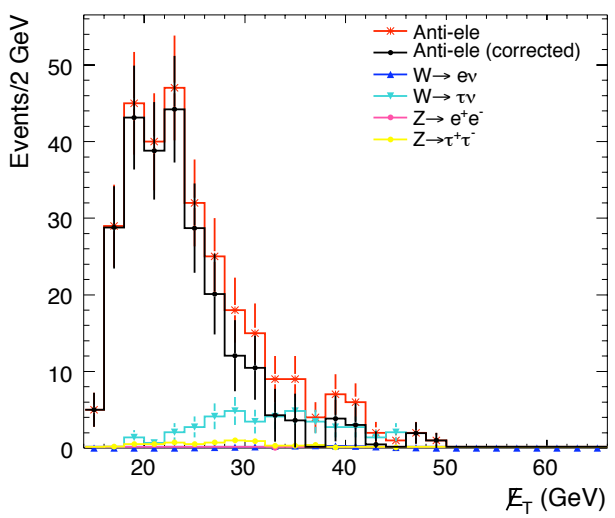

(d)

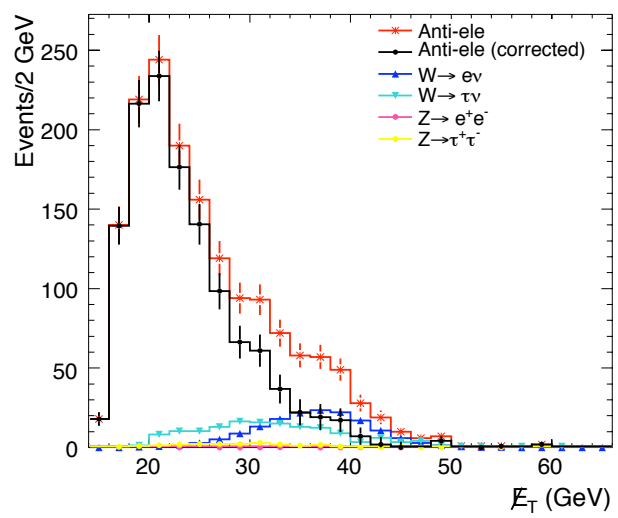

(e)

Figure 9.6: The $\mathbb{E}_{T}$ distribution in the 'anti-electron' sample before and after correcting for contamination from electroweak backgrounds where the following 'anti-electron' cuts have been used in the selection criteria: (a) default $E_{\text {had }} / E_{\text {em }}$ and $Q \Delta x$, (b) default $L_{\text {shr }}, \chi_{\text {strip }}^{2}$ and $E_{\text {had }} / E_{\text {em }}$, (c) at least 2 or more of the default cuts are required to pass, (d) exactly 3 cuts are required to pass where $E_{\text {had }} / E_{\text {em }}$ and $L_{\text {shr }}$ are set to their 'tight' values and the other cuts are set to their default values, (e) at least 2 or more of the 'tight' cuts are required to pass. 

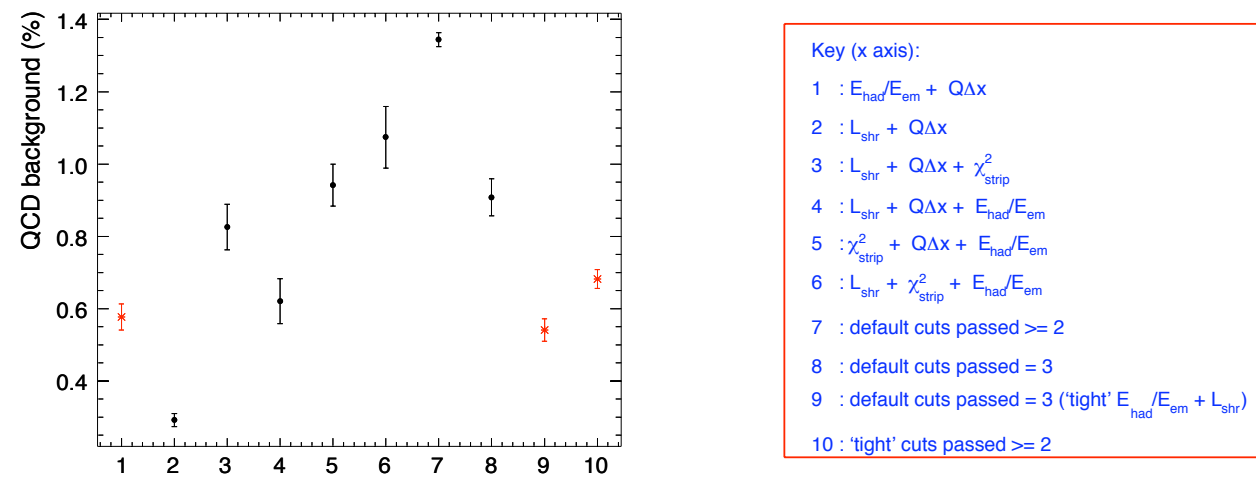

Figure 9.7: The fraction of QCD background obtained for different 'anti-electron' selection criteria. The points 1, 9 and 10 are those used in the final QCD background estimate.
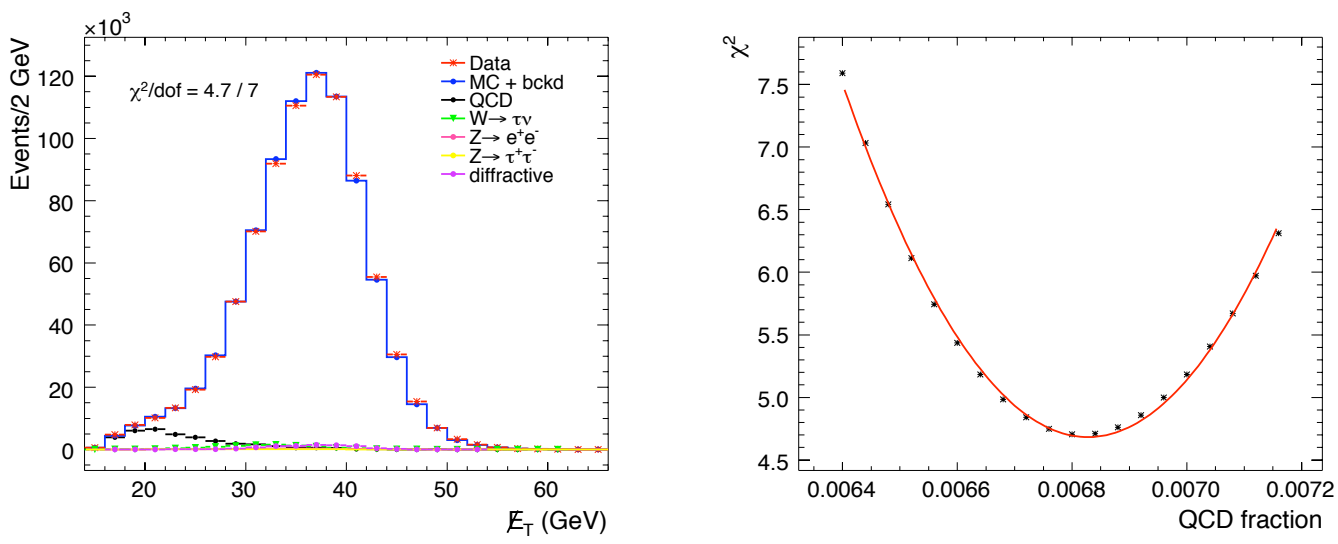

Figure 9.8: (a) Result of the $\not_{T}$ fit to data using a simulation $\not_{T}$ distribution comprising of the signal, QCD background and electroweak backgrounds. (b) The variation of the $\chi^{2}$ of the fit as a function of the amount of QCD background. 


\section{Chapter 10}

\section{Results}

\subsection{W Width}

The width of the $\mathrm{W}$ boson is obtained from fitting to the tail of the $M_{T}$ distribution. The fast simulation that has been described in detail in previous chapters is used to create simulation templates of the $M_{T}$ distribution with varying input $\Gamma_{W}$ values. A fit is then performed to the data $M_{T}$ distribution by normalising in the region $50<M_{T}<90$ $\mathrm{GeV}$ and fitting in the region $90<M_{T}<200 \mathrm{GeV}$. As mentioned before, the systematic uncertainties associated with modelling the $M_{T}$ distribution in the simulation are evaluated for the range $M_{T}^{\text {low }}<M_{T}<200 \mathrm{GeV}$, where $M_{T}^{\text {low }}=80,85,90,100$ and $110 \mathrm{GeV}$. The $M_{T}^{\text {low }}=90 \mathrm{GeV}$ value gives the smallest combined statistical and systematic uncertainty on the $\mathrm{W}$ width. Above this value, whereas the systematic uncertainties decrease, the statistical uncertainty increases due to the fewer events in the tail of the $M_{T}$ distribution. The background $M_{T}$ distributions are added to the simulation templates and the $\mathrm{W}$ width is extracted from a binned maximum-likelihood fit to the $M_{T}$ distribution in the data. The fit results are shown in Figure 10.1.

The breakdown of the systematic uncertainty and the total statistical uncertainty on the $\mathrm{W}$ width is shown in Table 10.1 for the electron and muon channels. An uncertainty on the $\mathrm{W}$ width from the value of the $\mathrm{W}$ boson mass used in the event generator is also included in the table. It is obtained by measuring the shift in the $\mathrm{W}$ width when the value of the $\mathrm{W}$ mass is varied within an uncertainty on the world average value of $25 \mathrm{MeV}$. It 
can be seen in Table 10.1 that the recoil contributes as the largest systematic on the W width in both the electron and muon channels. Since most of the systematic uncertainties are determined using fits to data, they are expected to decrease as the statistics of the data samples increase. This includes the recoil systematic which is constrained using the $\mathrm{Z} \rightarrow l^{+} l^{-}$samples as well as the energy and momentum scales and resolutions.

The $\mathrm{W}$ width is found to be

$$
\begin{gathered}
\Gamma_{W}=1948 \pm 67_{\text {stat. }} \pm 79_{\text {sys. }} \mathrm{MeV}(\mathrm{W} \rightarrow e \nu) \\
\Gamma_{W}=2118 \pm 60_{\text {stat. }} \pm 71_{\text {sys. }} \mathrm{MeV}(\mathrm{W} \rightarrow \mu \nu)
\end{gathered}
$$

The Best-Linear-Unbiased-Estimator (BLUE) [65] method is used to combine the result from the two channels taking into account all the correlations between systematics to give

$$
\Gamma_{W}=2033 \pm 73 \mathrm{MeV}
$$

which is in good agreement with the Standard Model prediction of $2093 \pm 2 \mathrm{MeV}$ and the current world average direct measurement (excluding this measurement) of $2147 \pm 60$ $\mathrm{MeV}[66]$. The measurement presented in this thesis is the world's best direct measurement of this quantity and represents a precision of less than $4 \%$ on the $\mathrm{W}$ width. This measurement can be combined with other direct measurements to give $2098 \pm 48 \mathrm{MeV}$ [24]. It is also in agreement with the indirect measurement of $2079 \pm 41 \mathrm{MeV}$ [39]. A summary of the values of the $\mathrm{W}$ width obtained from the Tevatron and LEP 2 experiments is given in Figure 10.2, where the CDF (Run II) result represents the measurement described in this thesis. 


\begin{tabular}{|l|cc|}
\hline & $\mathrm{W} \rightarrow e \nu$ & $\mathrm{W} \rightarrow \mu \nu$ \\
\hline \hline PDFs & 20 & 20 \\
Electroweak corrections & 10 & 6 \\
W boson $p_{\mathrm{T}}$ & 7 & 7 \\
W mass & 9 & 9 \\
Lepton ID & 10 & 6 \\
Acceptance & 3 & 4 \\
Momentum scale & & 17 \\
Momentum resolution & & 21 \\
Momentum non-gaussian & & 16 \\
Calorimeter scale & 17 & \\
Calorimeter resolution & 31 & \\
Calorimeter non-linearity & 12 & \\
Calorimeter material scale & 2 & \\
Calorimeter simulation & 13 & \\
Backgrounds & 32 & 33 \\
Recoil & 54 & 49 \\
\hline Statistics & 60 & 67 \\
\hline Total & 99 & 98 \\
\hline
\end{tabular}

Table 10.1: Summary of the systematic and statistical uncertainties, in $\mathrm{MeV}$, on the width of the W boson in the $\mathrm{W} \rightarrow e \nu$ and $\mathrm{W} \rightarrow \mu \nu$ decay channels.

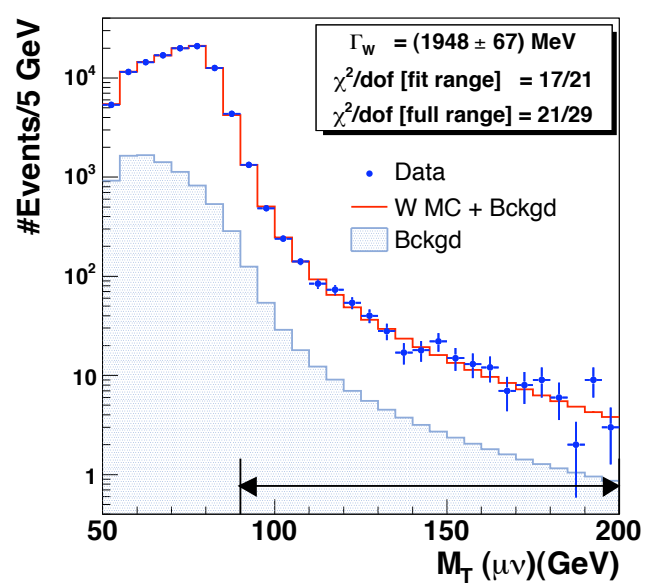

(a)

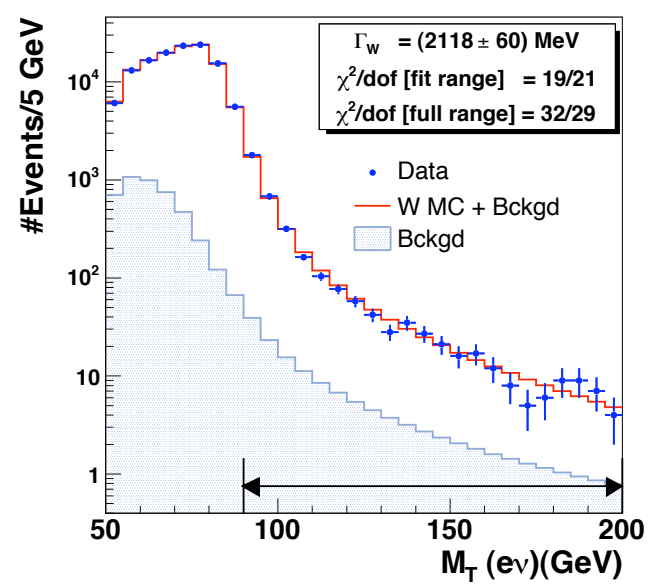

(b)

Figure 10.1: Distribution of $M_{T}$ for (a) W $\rightarrow e \nu$ and (b) W $\rightarrow \mu \nu$ events compared to the simulation for the $\mathrm{W}$ width analysis. 


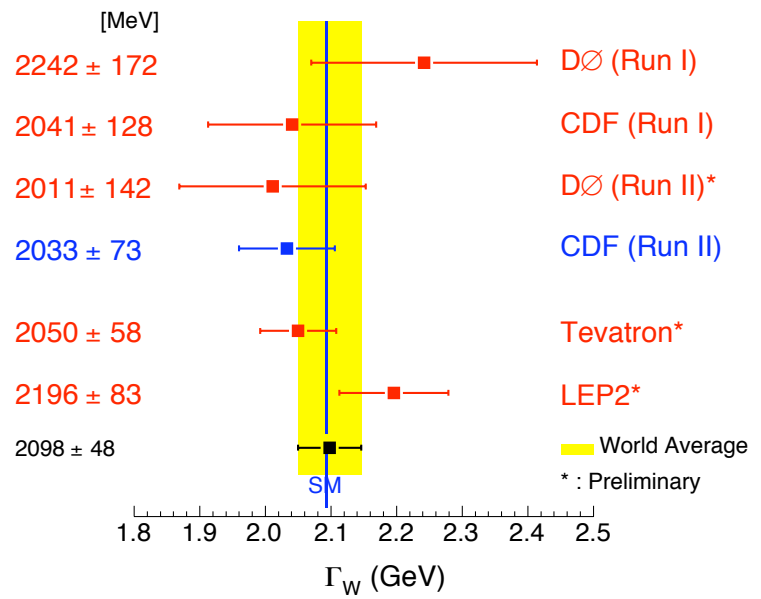

Figure 10.2: The width of the $\mathrm{W}$ boson as measured by the LEP and Tevatron experiments. The CDF (Run II) result is the measurement described in this thesis. The Tevatron and world average values include this result. 


\subsection{W Mass}

The contribution to the total systematic on the $\mathrm{W}$ mass from the statistical component of the recoil is $8 \mathrm{MeV}$ and $5 \mathrm{MeV}$ for the electron and muon channels respectively. It has been discussed in Chapter 7 that the discrepancy in the $U$ distribution and a discrepancy in the low $\Sigma E_{\mathrm{T}}$ region contribute $5(7) \mathrm{MeV}$ and 7 (10) $\mathrm{MeV}$ for the electron (muon) channels respectively. In addition, obtaining the recoil parameters from tuning only on the $\mathrm{CC} \mathrm{Z}$ events contributes an additional systematic of $6 \mathrm{MeV}$. The contribution from these various sources of systematics is shown in Table 10.2, where it has been assumed that the $6 \mathrm{MeV}$ systematic from only considering $\mathrm{CC} \mathrm{Z}$ events is the same for the muon channel. It was shown in Chapter 7 that the $E_{T}$ distribution is well described by the simulation despite the discrepancy in the $U$ distribution. Figures 10.3 and 10.4 show a comparison of the $M_{T}$ distribution in data and simulation for $\mathrm{W} \rightarrow e \nu$ and $\mathrm{W} \rightarrow \mu \nu$ events respectively. They show that the $M_{T}$ distribution is well described by the simulation.

Table 10.3 shows some of the systematic uncertainties on the $\mathrm{W}$ mass in the electron channel from the first CDF Run II measurement [22] of this quantity which used $200 \mathrm{pb}^{-1}$ of data. The recoil uncertainty for the $200 \mathrm{pb}^{-1}$ measurement has been obtained by adding in quadrature the recoil scale $(9 \mathrm{MeV})$ and recoil resolution $(7 \mathrm{MeV})$ uncertainties [22]. The systematic uncertainties shown in the table are statistics dependent and are therefore expected to scale with the statistics of the data sample. Table 10.3 also shows the projected systematic uncertainties for the electron channel from the various contributions using the dataset of $2400 \mathrm{pb}^{-1}$ described in this thesis, assuming that the systematics scale with statistics. It is evident that in the previous W mass measurement the dominant systematic uncertainty on $M_{W}$ was from the lepton energy scale, followed by the recoil. For the current measurement, the measured recoil systematic of $14 \mathrm{MeV}$ for the electron channel is much larger than the expected recoil systematic of $3 \mathrm{MeV}$, making it the dominant systematic. The combined recoil systematic of $13 \mathrm{MeV}$ for the electron and muon channels is also 
larger than the $11 \mathrm{MeV}$ recoil systematic for the previous measurement. However, it is expected that with a better description of the $U$ distribution and an understanding of the discrepancy in the low $\Sigma E_{\mathrm{T}}$ region, this systematic will be reduced. Several alternative functional forms for the $\Sigma E_{\mathrm{T}}$ description and the recoil have been tried but none produced a significant improvement in the agreement in the $U$ distribution and the low $\Sigma E_{\mathrm{T}}$ region. However, given more time, it is likely that the issues in these distributions will be resolved, thereby reducing the overall systematic on the $\mathrm{W}$ mass from the recoil.

\begin{tabular}{|l|cc|}
\hline & $\mathrm{W} \rightarrow e \nu$ & $\mathrm{W} \rightarrow \mu \nu$ \\
\hline \hline Z fit statistics & 8 & 5 \\
\hline$U$ discrepancy & 5 & 7 \\
low $\Sigma E_{\mathrm{T}}$ discrepancy & 7 & 10 \\
CC only & 6 & 6 \\
$\sigma\left(U_{2}\right)$ discrepancy & 5 & - \\
\hline luminosity discrepancy & 2.9 & 3.5 \\
\hline Total & 14 & 15 \\
\hline
\end{tabular}

Table 10.2: Summary of the systematic uncertainties, in $\mathrm{MeV}$, on $M_{W}$ from the recoil, including the uncertainty from the $\mathrm{Z}$ fit statistics, the discrepancy in the $U$ distribution and the $\sigma\left(U_{2}\right)$ distribution, the discrepancy in the low $\Sigma E_{\mathrm{T}}$ distribution and tuning the recoil only on CC events.

\begin{tabular}{|l|cc|}
\hline & $\begin{array}{c}200 \mathrm{pb}^{-1} \\
\text { dataset }\end{array}$ & $\begin{array}{c}2400 \mathrm{pb}^{-1} \\
\text { dataset (projected) }\end{array}$ \\
\hline \hline Energy scale & 30 & 8 \\
Momentum resolution & 9 & 3 \\
Backgrounds & 8 & 2 \\
Recoil & 11 & 3 \\
Statistics & 48 & 14 \\
\hline
\end{tabular}

Table 10.3: Summary of some of the systematic uncertainties on $M_{W}$ in the electron channel as measured in the previous W mass measurement and the projected systematic uncertainties for the current measurement. 


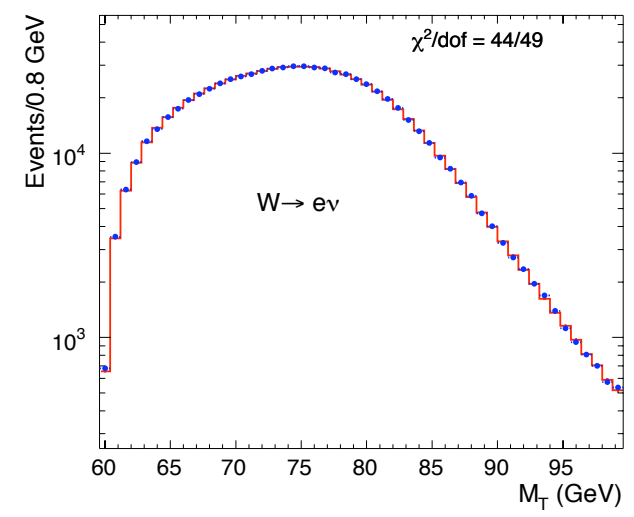

(a)

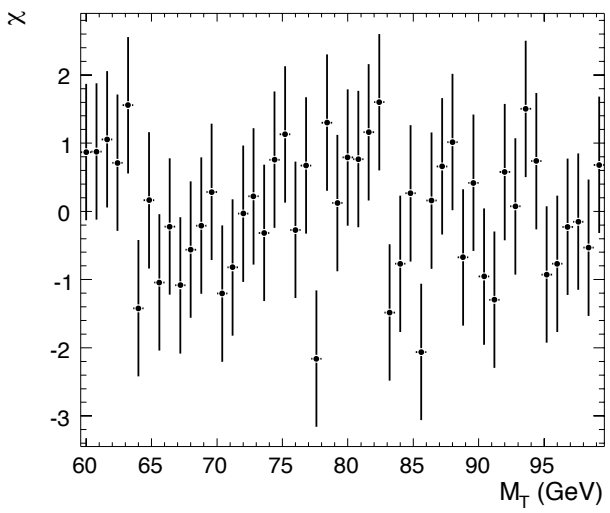

(b)

Figure 10.3: (a) Distribution of $M_{T}$ for $\mathrm{W} \rightarrow e \nu$ events compared to the simulation using the best fit recoil model parameters and (b) the $\chi$ plot for the comparison between data and simulation.

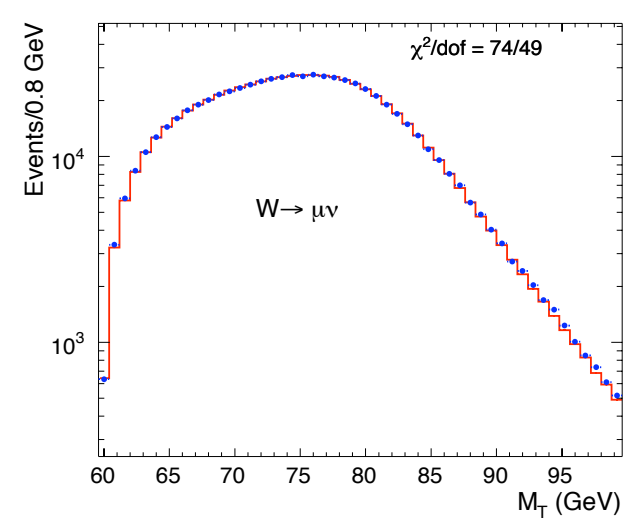

(a)

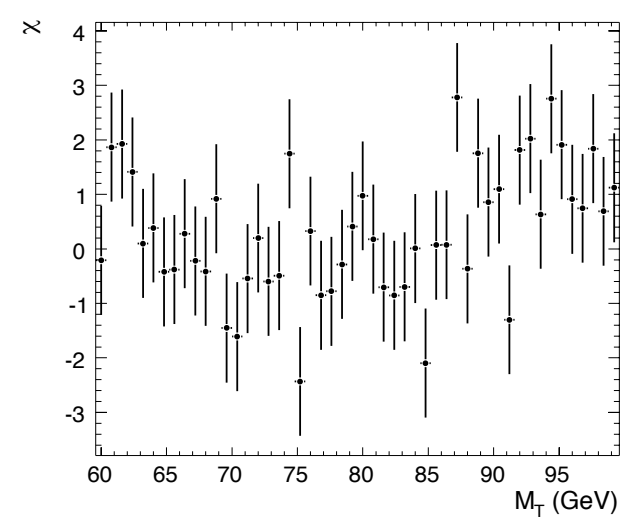

(b)

Figure 10.4: (a) Distribution of $M_{T}$ for $\mathrm{W} \rightarrow \mu \nu$ events compared to the simulation using the best fit recoil model parameters and (b) the $\chi$ plot for the comparison between data and simulation. 


\subsection{Summary}

The $\mathrm{W}$ width measurement was performed using approximately $350 \mathrm{pb}^{-1}$ of CDF Run II data. The dominant uncertainties contributing to the $\mathrm{W}$ width are the recoil, calorimeter resolution and backgrounds. The measurement produced the world's most precise measurement of this quantity with a total systematic uncertainty of $73 \mathrm{MeV}$, a precision of less than $4 \%$.

The $\mathrm{W}$ mass measurement is using approximately $2350 \mathrm{pb}^{-1}$ of CDF Run II data. The systematic uncertainties associated with the recoil have been evaluated and are larger than the uncertainty predicted assuming that the uncertainties from the previous $\mathrm{W}$ mass measurement scale directly with statistics. There are also uncertainties on the $\mathrm{W}$ mass resulting from discrepancies in various recoil distributions. It is clearly of importance to understand the discrepancies in these distributions as they translate to a shift in the fitted $\mathrm{W}$ mass. However, considering only the $\mathrm{Z}$ statistics uncertainty, it is evident from Table 10.3 that if the other systematics such as energy scale and momentum resolution scale directly with statistics, the recoil will be one of the dominant uncertainties on the $\mathrm{W}$ mass and there is continuing effort in understanding and reducing the recoil systematic. It is likely that once these systematics are reduced, the limiting systematics in future measurements may be those involved in the event generation, the key ones being PDFs and QED, which have large components that are dependent on theoretical calculations and do not scale directly with statistics. These systematics contributed approximately $11 \mathrm{MeV}$ and $12 \mathrm{MeV}$ respectively to the uncertainty on the $\mathrm{W}$ mass in the previous measurement. 


\section{References}

[1] F. Abe et al. Observation of top quark production in $p \bar{p}$ collisions, Physical Review Letters, 74:2626, (1995).

[2] S. Abachi et al. Observation of the top quark, Physical Review Letters, 74:2632, (1995).

[3] G. Arnison et al. Experimental Observation of Isolated Large Transverse Energy Electrons with Associated Missing Energy at $\sqrt{s}=540 \mathrm{GeV}$, Physics Letters B, 122: $103,(1983)$.

[4] M. Banner et al, Physics Letters B, 122:476, (1983).

[5] S. Glashow, Partial-Symmetries of Weak Interactions, Nuclear Physics, 22:579, (1961).

[6] A. Salam et al. Electromagnetic and Weak Interactions, Physics Letters, 13:168, (1964).

[7] S. Weinberg, A Model of Leptons, Physical Review Letters, 19:1264, (1967).

[8] P. Higgs, Broken Symmetries and the Masses of the Gauge Bosons, Physical Review Letters, 13:508, (1964).

[9] N. Cabbibo, Physical Review Letters, 10:531, (1963).

[10] M. Kobayashi and T. Maskawa, Progress in Theoretical Physics, 49:652, (1973).

[11] D. G. Michael et al. Observation of Muon Neutrino Disappearance with the MINOS Detectors in the NuMI Neutrino Beam, Physical Review Letters, 97:191801, (2006). 
[12] W. de Boer, Grand Unified Theories and Supersymmetry in Particle Physics and Cosmology, Progress in Particle and Nuclear Physics, 33:201, (1994).

[13] The CDF collaboration. The CDF II Detector Technical Design Report, FERMILABPub-96/390-E, (1996).

[14] T. Nelson. The CDF-II silicon tracking system, Nuclear Instruments and Methods A, 485:249, (2002).

[15] T. Affolder, D. Allspach, D. Ambrose and J. Bialek. CDF Central Outer Tracker, Nuclear Instruments and Methods A, 526:249, (2004).

[16] L. Balka et al. The CDF central electromagnetic calorimeter, Nuclear Instruments and Methods A, 267:272, (1988).

[17] S. Bertolucci et al. The CDF central and endwall hadron calorimeter, Nuclear Instruments and Methods A, 267:301, (1988).

[18] R. S. Lu CDF Collaboration. Operation aspects of CDF tracking and vertexing detectors, Nuclear Instruments and Methods A, 566:114, (2006).

[19] G. Ascoli et al CDF Central Muon Detector, Nuclear Instruments and Methods A, 268:33, (1988).

[20] E. J. Thomson et al, IEEE Transactions on Nuclear Science, 49:1063, (2002).

[21] D. C. Kennedy and B. W. Lynn, Nuclear Physics B, 322:1, (1989).

[22] T. Aaltonen et al. First Run II measurement of the W Boson Mass, Physical Review D, 77:112001, (2008).

[23] W. M. Yao et al. Review of Particle Physics, Journal of Physics G, 33, (2006).

[24] Tevatron Electroweak Working Group. Combination of CDF and D0 results on the W boson mass and width, arXiv:0808.0147v1, (2008).

[25] Tevatron Electroweak Working Group, Updated Combination of CDF and DØ Results for the Mass of the W Boson, arXiv:0908.1374v1.

[26] B. Abbott et al., Measurement of the W boson mass, Physical Review D, 58:092003, (1998). 
[27] Combination of the CDF and DØ Results on the Mass of the Top Quark, arXiv:0808.1089v1, (2008).

[28] Search for the Standard Model Higgs Boson at LEP, arXiv:hep-ex/0306033v1, 2003.

[29] The ALEPH, DELPHI, L3, OPAL, SLD Collaborations, the LEP Electroweak Working Group, the SLD Electroweak and Heavy Flavour Groups, Physics Reports, 427:257, (2006).

[30] S. Schael et al, Measurement of the $\mathrm{W}$ boson Mass and Width in $e^{+} e^{-}$Collisions at LEP, arXiv:hep-ex/0605011, (2006).

[31] P. Achard et al, Measurement of the mass and width of the W boson at LEP, arXiv:hep-ex/0511049, (2005).

[32] G. Abbiendi et al, Measurement of the mass and width of the W boson, arXiv:hepex/0508060, (2005).

[33] J. Abdallah et al, Measurement of the mass and width of the $\mathrm{W}$ boson in $e^{+} e^{-}$ collisions at $\sqrt{\hat{s}}=161-209 \mathrm{GeV}$, arXiv:0803.2534, (2007).

[34] The LEP Collaborations ALEPH, DELPHI, L3, OPAL, and the LEP Electroweak Working Group, A Combination of Preliminary Electroweak Measurements and Constraints on the Stan- dard Model, Eprint hep-ex/0612034, CERN, (2006).

[35] V. Abazov et al., Measurement of the W boson mass, to be submitted to Physical Review Letters.

[36] The LEP Electroweak Working Group, http://lepewwg.web.cern.ch/LEPEWWG/plots/summer2009/

[37] R. K. Ellis, W. J. Stirling, B. R. Webber, QCD and collider physics.

[38] J. Collins et al, Back-to-back jets in QCD, Nuclear Physics B, 193:381, (1981).

[39] D. Acosta et al, First Measurements of Inclusive W and Z Cross Sections from Run II of the Fermilab Tevatron Collider, Physical Review Letters, 94:091803, (2005).

[40] J. C. Collins and D. E. Soper, Physical Review D, 16:2219, (1977).

[41] E. Mirkes, Nuclear Physics B, 387:3, (1992). 
[42] UA2 TOYGEN Monte Carlo, unpublished.

[43] F. A. Berends, R. Kleiss, J. P. Revol, J. P. Vialle, Zeitschrift fur Physik C 27:155, (1985); F. A. Berends, R. Kleiss, Zeitschrift fur Physik C, 27:365, (1985).

[44] J. L. Rosner and M. P. Worah. Oblique corrections to the W Width, Physical Review D, 49:1363, (1994).

[45] J. Pumplin, D. R. Stump, J. Huston, H. L. Lai, P. Nadolsky and W. K. Tung, JHEP, 0207:012, (2002).

[46] A. D. Martin, R. G. Roberts, W. J. Stirling and R. S. Thorne, European Physics Journal C, 4:463, (1998).

[47] A. D. Martin, R. G. Roberts, W. J. Stirling Parton distributions: a study of the new HERA data, $\alpha_{s}$, the gluon and $p \bar{p}$ jet production, Physics Letters B, 4:419, (1996).

[48] E. Barberio, Z. Was, Computer Physics Communications, 79:291, (1994); E. Barberio, B. van Eijik, Z. Was, Computer Physics Communications, 66:115, (1991).

[49] C. Balazs, C. P. Yuan, Physical Review D, 56:5558, (1997).

[50] P. B. Arnold, R. P. Kauffman, Nuclear Physics B, 349:38, (1991);

P. B. Arnold, M. Reno, Nuclear Physics B, 319:37, (1989);

P. B. Arnold, M. Reno, R. K. Ellis, Physical Review D, 40:912, (1989)

[51] F. Landry, R. Brock, P. M. Nadolsky, and C. P. Yuan, Physical Review D, 67:073016, (2003).

[52] J. Collins, D. Soper and G. Sterman. Transverse momentum distribution in Drell-Yan pair and W and Z boson production, Nuclear Physics B, 250:199, (1985).

[53] P. Aronold and R. Kauffman. W and Z production at next-to-leading order: From large $q_{T}$ to small, Nuclear Physics B, 349:381, (1991).

[54] C. M. Carloni Calame, G. Montagna, O. Nicrosini and A. Vicini, Precision electroweak calculations of the production of a high transverse-momentum lepton pair at hadron colliders, JHEP 0710:109, (2007) (arXiv:0710.1722 [hep-ph])

[55] M. Lancaster, CDF-NOTE-4770 (1998).

[56] U. Baur and D. Wackeroth, Physical Review D, 70:073015, (2004). 
[57] C. Amsler et al., Physics Letters B, 667:1, (2008) Eq. 27.1

[58] R. M. Sternheimer, Physics Review, 88:851, (1952).

[59] J. H. Hubbell et al Atomic Form Factors, Incoherent Scattering Functions and Photon Scattering Cross Sections, Journal of Physical and Chemical Reference Data, 4:471, (1975).

[60] GEANT 3 Detector Description and Simulation Tool. CERN Program Library Long Writeup W5013, (1993).

[61] Troy Vine, A Direct Measurement of the W Decay Width, PhD thesis, University College London, (2008).

[62] K. Rinnert, A Fast-Access Material Map for the SVX II/ISL Silicon Tracking Volume at CDF 2, CDF Note 5846 (2002).

[63] W. L. van Neerven and E. B. Zijlstra. The $\mathrm{O}\left(\alpha_{s}^{2}\right)$ corrected Drell-Yan K-factor in the DIS and MS schemes, Nuclear Physics B, 382:11, (2002).

[64] T. Sjostrand et al., PYTHIA 6.2 Physics and Manual, hep-ph/0108264 (2001).

[65] P. Clifford, L. Lyons, D. Gibaut. How to combine correlates estimates of a single physical quantity, Nuclear Instruments and Methods A, 270:110, (1988).

[66] The LEP Collaborations. A Combination of Preliminary Electroweak Measurements and Constraints on the Standard Model. arXiv:hep-ex/0612034v2, (2007).

[67] M. E. Convery, Diffractive and Exclusive Production at the Tevatron, arXiv:0810.2519. 